\title{
Kinetic Resolution of 2,2-Disubstituted Dihydroquinolines through Chiral Phosphoric Acid Catalyzed C6-selective Asymmetric Halogenations
}

Chaofan Zhu ${ }^{\mathrm{a}, \mathrm{b}}$, Wei Liu ${ }^{\mathrm{b}}$, Fei Zhao ${ }^{\mathrm{c}}$, Yunrong Chen $^{\mathrm{b}, *}$, Houchao Tao ${ }^{\mathrm{c}, *}$, Yu-Peng $\mathrm{He}^{\mathrm{a}, *}$ and Xiaoyu Yang ${ }^{\mathrm{b}, *}$

${ }^{a}$ Key Laboratory of Petrochemical Catalytic Science and Technology, Liaoning Shihua University, Fushun 113001, China

${ }^{\mathrm{b}}$ School of Physical Science and Technology, ShanghaiTech University, Shanghai 201210, China

ciHuman Institute, ShanghaiTech University, Shanghai 201210, China 


\section{Table of Contents}

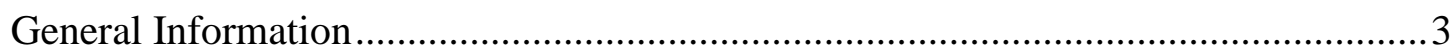

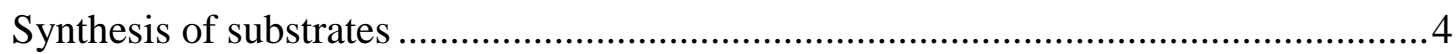

Kinetic resolution of DHQs through asymmetric bromination..................................12

Kinetic resolution of DHQ through asymmetric iodonation.....................................28

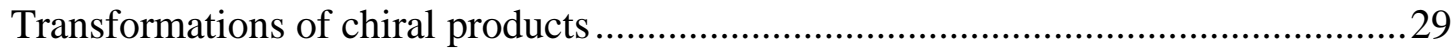

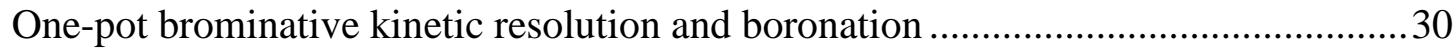

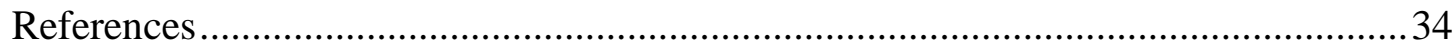

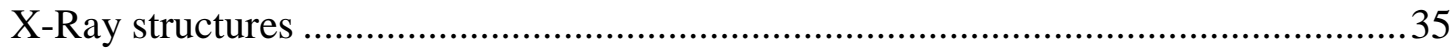

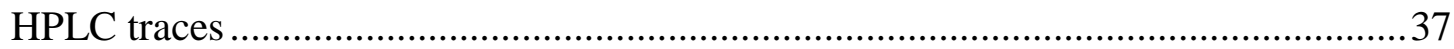

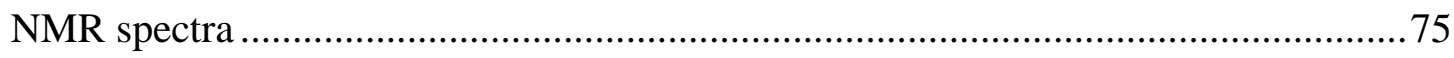




\section{General Information:}

Unless otherwise noted, all commercial reagents were used without further purification. Dichloromethane, toluene, ether, THF were purified by passage through an activated alumina column under argon. Thin-layer chromatography (TLC) analysis of reaction mixtures were performed using Huanghai silica gel HSGF254 TLC plates, and visualized under UV or by staining with ceric ammonium molybdate or potassium permanganate. Flash column chromatography was carried out on Huanghai Silica Gel HHGJ-300, 300-400 mesh. Nuclear magnetic resonance (NMR) spectra were recorded using Bruker Avance III HD spectrometer (FT, $400 \mathrm{MHz}$ for ${ }^{1} \mathrm{H}, 101 \mathrm{MHz}$ for ${ }^{13} \mathrm{C}$ ). ${ }^{1} \mathrm{H}$ and ${ }^{13} \mathrm{C}$ chemical shifts are reported in ppm downfield of tetramethylsilane and referenced to residual solvent peak $\left(\mathrm{CHCl}_{3} ; \delta \mathrm{H}=7.26\right.$ and $\delta \mathrm{C}=77.16, \mathrm{CD}_{3} \mathrm{OD}, \delta \mathrm{H}=3.31$ and $\delta \mathrm{C}=49.00,\left(\mathrm{CD}_{3}\right)_{2} \mathrm{CO}, \delta \mathrm{H}=2.05$ and $\left.\delta \mathrm{C}=29.84\right)$. Multiplicities are reported using the following abbreviations: $\mathrm{s}=$ singlet, $\mathrm{d}=$ doublet, $\mathrm{t}=$ triplet, $\mathrm{q}=$ quartet, $\mathrm{m}=$ multiplet, $\mathrm{br}=$ broad resonance. FT-IR spectras were recorded on PerkinElmer Frontier FT-IR Spectrometer, and absorption frequencies are reported in reciprocal centimeters $\left(\mathrm{cm}^{-1}\right)$. Mass spectral data were obtained from the Agilent Technologies 6230 TOF LC/MS spectrometer in electrospray ionization $\left(\mathrm{ESI}^{+}\right)$mode. Optical rotations were measured with an Autopol V Plus/VI digital polarimeter. X-Ray structure analyses were performed using a Bruker D8 Venture X-ray single crystal diffractometer. Enantiomeric excesses were determined on an Agilent 1260 Chiral HPLC using IA, IB, IC, ID, IG columns under the detective wavelength of $254 \mathrm{~nm}$. The racemic products were synthesized by using ( \pm )-A1 as catalyst. 


\section{Synthesis of substrates}

\section{Method A:}<smiles>Brc1ccc2ccccc2n1</smiles>
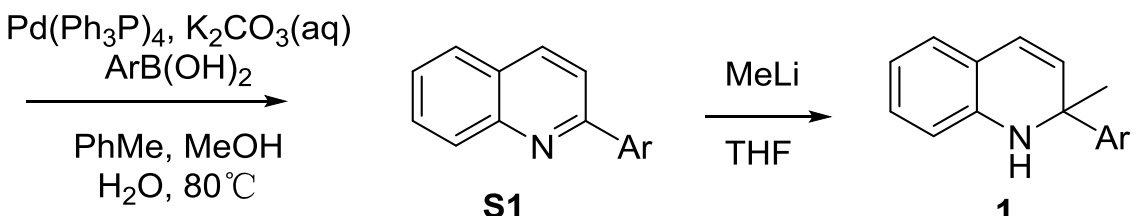

Substrates 1a to 1k, 1p-1q were synthesized with $\operatorname{method} \mathbf{A}^{\mathbf{1}}$.

To a mixture of 2-bromoquinoline (15.0 mmol), arylboronic acid (22.5 mmol, 1.5 equiv.) and $\mathrm{Pd}\left(\mathrm{Ph}_{3} \mathrm{P}\right)_{4}(866 \mathrm{mg}, 0.75 \mathrm{mmol}, 5 \mathrm{~mol} \%)$ in toluene $(105 \mathrm{~mL})$ and methanol $(18 \mathrm{~mL})$ was added $\mathrm{K}_{2} \mathrm{CO}_{3}$ aqueous solution $\left(2.5 \mathrm{M}, 18 \mathrm{~mL}, 3.0\right.$ equiv.) idunder $\mathrm{N}_{2}$ atmosphere. After stirring at $80{ }^{\circ} \mathrm{C}$ (heated by an oil bath) for $2 \mathrm{~h}$, the mixture was cooled to room temperature and extracted with EtOAc for 3 times. The combined organic layer was dried over $\mathrm{Na}_{2} \mathrm{SO}_{4}$, filtered and concentrated in vacuo to give a residue, which was purified by flash chromatography (300 400 mesh silica gel, petroleum ether/ethyl acetate $=40: 1)$ to afford the 2-aryl quinoline S1.

To a solution of $\mathbf{S 1}(1.0 \mathrm{mmol})$ in dry THF $(5 \mathrm{~mL})$ was added MeLi $(2.5 \mathrm{M}, 1.2 \mathrm{~mL}, 3.0$ mmol, 3.0 equiv.) dropwisely at $0{ }^{\circ} \mathrm{C}$. After stirring at $\mathrm{rt}$ for $6 \mathrm{~h}$, the mixture was quenched with saturated $\mathrm{NH}_{4} \mathrm{Cl}$ aqueous solution at $0{ }^{\circ} \mathrm{C}$, diluted with EtOAc $(30 \mathrm{~mL})$ and washed with brine $(15 \mathrm{~mL} \times 2)$. The organic layer was dried over $\mathrm{Na}_{2} \mathrm{SO}_{4}$, filtered and concentrated in vacuo to give a residue, which was purified by flash chromatography (300 400 mesh silica gel, petroleum ether/dichloromethane $=6: 1)$ to afford the product 1 .

\section{Method B}

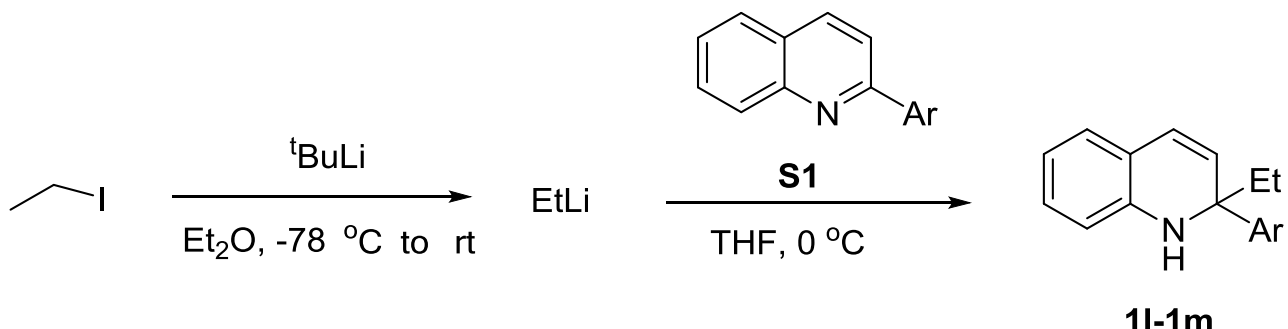

Substrates 11-1m were synthesized with method $\mathbf{B}^{2}$ 
To a solution of EtI (3 mmol, 1equiv.) in $\mathrm{Et}_{2} \mathrm{O}(6 \mathrm{~mL})$ was added ${ }^{\mathrm{t}} \mathrm{BuLi}(6 \mathrm{~mL}, 1.25 \mathrm{M}, 2.5$ equiv.) dropwisely at $-78{ }^{\circ} \mathrm{C}$. After stirring for $2 \mathrm{~h}$ at this temperature, the mixture was warmed to rt and stirred for another $1 \mathrm{~h}$ to give the solution of EtLi.

To a mixture of 2-aryl quinoline $\mathbf{S 1}(200 \mathrm{mg}, 1 \mathrm{mmol})$ in dry THF ( $5 \mathrm{~mL})$ was added the above solution of EtLi (3 equiv.) dropwisely at $0{ }^{\circ} \mathrm{C}$. After stirring at $\mathrm{rt}$ for $6 \mathrm{~h}$, the mixture was quenched with saturated $\mathrm{NH}_{4} \mathrm{Cl}$ aqueous solution at $0{ }^{\circ} \mathrm{C}$ and extracted with EtOAc for 3 times. The combined organic layer was washed with brine, dried over $\mathrm{Na}_{2} \mathrm{SO}_{4}$, filtered and concentrated in vacuo to give a residue, which was purified by flash chromatography (300 400 mesh silica gel, petroleum ether/dichloromethane $=6: 1)$ to afford the product 1l-1m.

2-methyl-2-phenyl-1,2-dihydroquinoline (1a)<smiles>CC1(c2ccccc2)C=Cc2ccccc2N1</smiles>

Yellow oil, $121 \mathrm{mg}$, 55\% yield from S1 (205 mg), purified by column chromatography (petroleum ether:DCM = 6:1). ${ }^{1} \mathrm{H}$ NMR $(400 \mathrm{MHz}$, Chloroform- $d$ ) $\delta 7.62-7.47(\mathrm{~m}, 2 \mathrm{H})$, $7.35(\mathrm{t}, \mathrm{J}=7.7 \mathrm{~Hz}, 2 \mathrm{H}), 7.23(\mathrm{~d}, \mathrm{~J}=7.3 \mathrm{~Hz}, 1 \mathrm{H}), 7.00(\mathrm{td}, \mathrm{J}=7.6,1.6 \mathrm{~Hz}, 1 \mathrm{H}), 6.91(\mathrm{dd}, \mathrm{J}=$ 7.4, $1.7 \mathrm{~Hz}, 1 \mathrm{H}), 6.60$ (t, J = 7.4 Hz, 1H), $6.45(\mathrm{~d}, \mathrm{~J}=7.9 \mathrm{~Hz}, 1 \mathrm{H}), 6.35$ (d, J = 9.7 Hz, 1H), $5.60(\mathrm{~d}, \mathrm{~J}=9.7 \mathrm{~Hz}, 1 \mathrm{H}), 4.05(\mathrm{~s}, 1 \mathrm{H}), 1.74(\mathrm{~s}, 3 \mathrm{H}) .{ }^{13} \mathrm{C}$ NMR $(101 \mathrm{MHz}$, Chloroform- $d) \delta$ $149.1,142.7,129.9,129.1,128.5,127.0,127.0,125.5,123.5,119.3,117.3,112.5,57.5,30.3$. HRMS (ESI) m/z: $[\mathrm{M}+\mathrm{H}]^{+}$Calcd for $\mathrm{C}_{16} \mathrm{H}_{16} \mathrm{~N}^{+}$222.1277; Found 222.1274.

2-methyl-2-(p-tolyl)-1,2-dihydroquinoline (1b)<smiles>Cc1ccc(C2(C)C=Cc3ccccc3N2)cc1</smiles>

Yellow solid, $106 \mathrm{mg}$, 45\% yield from S1 (220 mg), purified by column chromatography (petroleum ether:DCM = 6:1). ${ }^{1} \mathrm{H}$ NMR $(400 \mathrm{MHz}$, Chloroform- $d$ ) $\delta 7.41(\mathrm{~d}, J=8.2 \mathrm{~Hz}, 2 \mathrm{H})$, $7.15(\mathrm{~d}, J=7.9 \mathrm{~Hz}, 2 \mathrm{H}), 6.99(\mathrm{td}, J=7.6,1.5 \mathrm{~Hz}, 1 \mathrm{H}), 6.90(\mathrm{dd}, J=7.4,1.5 \mathrm{~Hz}, 1 \mathrm{H}), 6.58(\mathrm{t}$, 
$J=7.4 \mathrm{~Hz}, 1 \mathrm{H}), 6.44(\mathrm{~d}, J=7.9 \mathrm{~Hz}, 1 \mathrm{H}), 6.33(\mathrm{~d}, J=9.7 \mathrm{~Hz}, 1 \mathrm{H}), 5.58(\mathrm{~d}, J=9.7 \mathrm{~Hz}, 1 \mathrm{H})$, $4.02(\mathrm{~s}, 1 \mathrm{H}), 2.33(\mathrm{~s}, 3 \mathrm{H}), 1.71(\mathrm{~s}, 3 \mathrm{H}) .{ }^{13} \mathrm{C}$ NMR (101 MHz, Chloroform- $d$ ) $\delta$ 146.3, 142.7, 136.6, 130.1, 129.2, 129.0, 127.0, 125.4, 123.3, 119.3, 117.2, 112.5, 57.3, 30.3, 21.1. HRMS (ESI) $\mathrm{m} / \mathrm{z}:[\mathrm{M}+\mathrm{H}]^{+}$Calcd for $\mathrm{C}_{17} \mathrm{H}_{18} \mathrm{~N}^{+}$236.1434; Found 236.1429.

3-(4-(tert-butyl)phenyl)-2-methyl-1,2-dihydroquinoline (1c)<smiles>CC(C)(C)c1ccc(C2(C)C=Cc3ccccc3N2)cc1</smiles>

Green solid, $125 \mathrm{mg}$, 45\% yield from S1 (260 mg), purified by column chromatography (petroleum ether:DCM = 6:1). ${ }^{1} \mathrm{H}$ NMR $(500 \mathrm{MHz}$, Chloroform- $d$ ) $\delta 7.46(\mathrm{~d}, J=8.5 \mathrm{~Hz}, 2 \mathrm{H})$, $7.36(\mathrm{~d}, J=8.5 \mathrm{~Hz}, 2 \mathrm{H}), 6.99(\mathrm{td}, J=7.6,1.6 \mathrm{~Hz}, 1 \mathrm{H}), 6.91(\mathrm{dd}, J=7.5,1.5 \mathrm{~Hz}, 1 \mathrm{H}), 6.59$ (t, $J=7.4 \mathrm{~Hz}, 1 \mathrm{H}), 6.43(\mathrm{~d}, J=7.9 \mathrm{~Hz}, 1 \mathrm{H}), 6.33(\mathrm{~d}, J=9.7 \mathrm{~Hz}, 1 \mathrm{H}), 5.59(\mathrm{~d}, J=9.7 \mathrm{~Hz}, 1 \mathrm{H})$, $4.04(\mathrm{~s}, 1 \mathrm{H}), 1.72(\mathrm{~s}, 3 \mathrm{H}), 1.31$ (s, 9H). ${ }^{13} \mathrm{C}$ NMR (126 MHz, Chloroform- $d$ ) $\delta$ 150.1, 145.7, $141.7,131.3,131.2,129.2,125.5,125.2,122.2$, 121.1, 113.9, 108.5, 57.4, 34.6, 31.5, 30.1. HRMS (ESI) m/z: $[\mathrm{M}+\mathrm{H}]^{+}$Calcd for $\mathrm{C}_{20} \mathrm{H}_{24} \mathrm{~N}^{+}$278.1903; Found 278.1899.

2-([1,1'-biphenyl]-4-yl)-2-methyl-1,2-dihydroquinoline (1d)<smiles>CC1(c2ccc(-c3ccccc3)cc2)C=Cc2ccccc2N1</smiles>

Green solid, $143 \mathrm{mg}$, 48\% yield from S1 $(280 \mathrm{mg}$ ), purified by column chromatography (petroleum ether:DCM = 4:1). ${ }^{1} \mathrm{H}$ NMR $(400 \mathrm{MHz}$, Chloroform- $d$ ) $\delta 7.58(\mathrm{q}, J=6.9 \mathrm{~Hz}, 6 \mathrm{H})$, $7.43(\mathrm{t}, J=7.6 \mathrm{~Hz}, 2 \mathrm{H}), 7.34(\mathrm{t}, J=7.4 \mathrm{~Hz}, 1 \mathrm{H}), 7.01(\mathrm{t}, J=7.6 \mathrm{~Hz}, 1 \mathrm{H}), 6.93(\mathrm{~d}, J=7.3 \mathrm{~Hz}$, 1H), $6.61(\mathrm{t}, J=7.4 \mathrm{~Hz}, 1 \mathrm{H}), 6.48(\mathrm{~d}, J=7.9 \mathrm{~Hz}, 1 \mathrm{H}), 6.37(\mathrm{~d}, J=9.7 \mathrm{~Hz}, 1 \mathrm{H}), 5.63(\mathrm{~d}, J=$ $9.7 \mathrm{~Hz}, 1 \mathrm{H}), 4.09(\mathrm{~s}, 1 \mathrm{H}), 1.77(\mathrm{~s}, 3 \mathrm{H}) .{ }^{13} \mathrm{C}$ NMR $(101 \mathrm{MHz}$, Chloroform- $d) \delta$ 148.2, 142.7, $140.9,139.9,129.8,129.1,128.9,127.4,127.3,127.2,127.0,126.0,123.6,119.3,117.4,112.5$, 57.4, 30.4. HRMS (ESI) m/z: $[\mathrm{M}+\mathrm{H}]^{+}$Calcd for $\mathrm{C}_{22} \mathrm{H}_{20} \mathrm{~N}^{+}$298.1590; Found 298.1586. 
2-(4-methoxyphenyl)-2-methyl-1,2-dihydroquinoline (1e)<smiles>COc1ccc(C2(C)C=Cc3ccccc3N2)cc1</smiles>

Yellow solid, $116 \mathrm{mg}, 46 \%$ yield from S1 $(235 \mathrm{mg}$ ), purified by column chromatography (petroleum ether:DCM = 6:1). ${ }^{1} \mathrm{H}$ NMR (500 MHz, Chloroform- $d$ ) $\delta 7.49-7.42(\mathrm{~m}, 2 \mathrm{H}), 6.99$ $(\mathrm{td}, J=7.6,1.5 \mathrm{~Hz}, 1 \mathrm{H}), 6.91(\mathrm{dd}, J=7.4,1.6 \mathrm{~Hz}, 1 \mathrm{H}), 6.89-6.84(\mathrm{~m}, 2 \mathrm{H}), 6.59(\mathrm{td}, J=7.4$, $1.1 \mathrm{~Hz}, 1 \mathrm{H}), 6.43(\mathrm{~d}, J=7.9 \mathrm{~Hz}, 1 \mathrm{H}), 6.33(\mathrm{~d}, J=9.7 \mathrm{~Hz}, 1 \mathrm{H}), 5.56(\mathrm{~d}, J=9.8 \mathrm{~Hz}, 1 \mathrm{H}), 4.0(\mathrm{~s}$, 1H), 3.79 (s, 3H), 1.71 (s, 3H). ${ }^{13} \mathrm{C}$ NMR (126 MHz, Chloroform-d) $\delta$ 158.4, 142.6, 141.4, 130.1, 128.9, 126.8, 126.7, 123.1, 119.1, 117.1, 113.7, 112.3, 56.9, 55.3, 30.1. HRMS (ESI) m/z: $[\mathrm{M}+\mathrm{H}]^{+}$Calcd for $\mathrm{C}_{17} \mathrm{H}_{18} \mathrm{NO}^{+}$252.1383; Found 252.1379.

3-methyl-2-(m-tolyl)-1,2-dihydroquinoline (1f)<smiles>Cc1cccc(C2(C)C=Cc3ccccc3N2)c1</smiles>

White solid, $117 \mathrm{mg}, 50 \%$ yield from S1 $(220 \mathrm{mg}$ ), purified by column chromatography (petroleum ether:DCM = 6:1). ${ }^{1} \mathrm{H}$ NMR $(400 \mathrm{MHz}$, Chloroform- $d$ ) $\delta 7.41-7.30(\mathrm{~m}, 2 \mathrm{H}), 7.23$ $(\mathrm{d}, J=7.6 \mathrm{~Hz}, 1 \mathrm{H}), 7.06(\mathrm{~d}, J=7.4 \mathrm{~Hz}, 1 \mathrm{H}), 7.00(\mathrm{td}, J=7.6,1.6 \mathrm{~Hz}, 1 \mathrm{H}), 6.91(\mathrm{dd}, J=7.5$, $1.6 \mathrm{~Hz}, 1 \mathrm{H}), 6.60(\mathrm{t}, J=7.4 \mathrm{~Hz}, 1 \mathrm{H}), 6.47(\mathrm{~d}, J=7.9 \mathrm{~Hz}, 1 \mathrm{H}), 6.34(\mathrm{~d}, J=9.7 \mathrm{~Hz}, 1 \mathrm{H}), 5.60$ $(\mathrm{d}, J=9.7 \mathrm{~Hz}, 1 \mathrm{H}), 2.36(\mathrm{~s}, 3 \mathrm{H}), 1.72(\mathrm{~s}, 3 \mathrm{H}) \cdot{ }^{13} \mathrm{C}$ NMR $(126 \mathrm{MHz}$, Chloroform- $d$ ) $\delta 148.9$, $138.0,129.9,128.9,128.3,127.6,126.9,126.3,123.3,122.4,119.2,117.3,112.5,57.4,30.2$, 21.7. HRMS (ESI) m/z: [M+H] $]^{+}$Calcd for $\mathrm{C}_{17} \mathrm{H}_{18} \mathrm{~N}^{+}$236.1434; Found 236.1423.

2-(3-methoxyphenyl)-2-methyl-1,2-dihydroquinoline (1g)<smiles>COc1cccc(C2(C)C=Cc3ccccc3N2)c1</smiles>

White solid, $116 \mathrm{mg}, 46 \%$ yield from S1 (235 mg), purified by column chromatography (petroleum ether:DCM = 6:1). ${ }^{1} \mathrm{H}$ NMR $(500 \mathrm{MHz}$, Chloroform- $d$ ) $\delta 7.24(\mathrm{~s}, 1 \mathrm{H}), 7.13-7.06$ 
(m, 2H), $6.99(\mathrm{t}, J=7.7 \mathrm{~Hz}, 1 \mathrm{H}), 6.90(\mathrm{~d}, J=7.4 \mathrm{~Hz}, 1 \mathrm{H}), 6.77(\mathrm{~d}, J=8.2 \mathrm{~Hz}, 1 \mathrm{H}), 6.59(\mathrm{t}, J$ $=7.5 \mathrm{~Hz}, 1 \mathrm{H}), 6.45(\mathrm{~d}, J=7.9 \mathrm{~Hz}, 1 \mathrm{H}), 6.33(\mathrm{~d}, J=9.7 \mathrm{~Hz}, 1 \mathrm{H}), 5.59(\mathrm{~d}, J=9.7 \mathrm{~Hz}, 1 \mathrm{H})$, $4.1(\mathrm{~s}, 1 \mathrm{H}), 3.78(\mathrm{~d}, J=2.1 \mathrm{~Hz}, 3 \mathrm{H}), 1.70(\mathrm{~d}, J=2.0 \mathrm{~Hz}, 3 \mathrm{H}) \cdot{ }^{13} \mathrm{C}$ NMR $(126 \mathrm{MHz}$, Chloroform- $d$ ) $\delta 159.8,150.8,142.7,129.8,129.5,129.0,127.0,123.6,119.3,117.8,117.4$, 112.5, 112.0, 111.8, 57.5, 55.3, 30.3. HRMS (ESI) m/z: $[\mathrm{M}+\mathrm{H}]^{+}$Calcd for $\mathrm{C}_{17} \mathrm{H}_{18} \mathrm{NO}^{+}$ 252.1383; Found 252.1380.

2-(3,5-dimethoxyphenyl)-2-methyl-1,2-dihydroquinoline (1h)<smiles>COc1cc(OC)cc(C2(C)C=Cc3ccccc3N2)c1</smiles>

White solid, $118 \mathrm{mg}$, 42\% yield from S1 (266 mg), purified by column chromatography (petroleum ether:DCM = 6:1). ${ }^{1} \mathrm{H}$ NMR $(500 \mathrm{MHz}$, Chloroform- $d$ ) $\delta 6.99(\mathrm{t}, J=7.6 \mathrm{~Hz}, 1 \mathrm{H})$, $6.90(\mathrm{~d}, J=7.4 \mathrm{~Hz}, 1 \mathrm{H}), 6.67(\mathrm{t}, J=1.6 \mathrm{~Hz}, 2 \mathrm{H}), 6.59(\mathrm{t}, J=7.4 \mathrm{~Hz}, 1 \mathrm{H}), 6.46(\mathrm{~d}, J=7.9 \mathrm{~Hz}$, $1 \mathrm{H}), 6.35-6.31(\mathrm{~m}, 2 \mathrm{H}), 5.59(\mathrm{~d}, J=9.7 \mathrm{~Hz}, 1 \mathrm{H}), 3.77(\mathrm{~s}, 6 \mathrm{H}), 1.68(\mathrm{~s}, 3 \mathrm{H}) .{ }^{13} \mathrm{C}$ NMR $(126$ MHz, Chloroform- $d$ ) $\delta 160.9,151.7,142.6,129.7,129.0,127.0,123.6,119.3,117.4,112.6$, 104.1, 98.3, 57.6, 55.5, 30.2. HRMS (ESI) m/z: $[\mathrm{M}+\mathrm{H}]^{+}$Calcd for $\mathrm{C}_{18} \mathrm{H}_{20} \mathrm{NO}_{2}{ }^{+}$282.1489; Found 282.1486.

2-(benzo[d][1,3]dioxol-5-yl)-2-methyl-1,2-dihydroquinoline (1i)<smiles>CC1(c2ccc3c(c2)OCO3)C=Cc2ccccc2N1</smiles>

Yellow solid, $127 \mathrm{mg}$, 48\% yield from S1 (250 mg), purified by column chromatography (petroleum ether:DCM = 6:1). ${ }^{1} \mathrm{H}$ NMR $(400 \mathrm{MHz}$, Chloroform- $d$ ) $\delta 7.05(\mathrm{~d}, J=1.9 \mathrm{~Hz}, 1 \mathrm{H})$, $7.02-6.93(\mathrm{~m}, 2 \mathrm{H}), 6.93-6.85(\mathrm{~m}, 1 \mathrm{H}), 6.76(\mathrm{~d}, J=8.1 \mathrm{~Hz}, 1 \mathrm{H}), 6.59(\mathrm{t}, J=7.4 \mathrm{~Hz}, 1 \mathrm{H})$, $6.43(\mathrm{~d}, J=7.9 \mathrm{~Hz}, 1 \mathrm{H}), 6.33(\mathrm{~d}, J=9.7 \mathrm{~Hz}, 1 \mathrm{H}), 5.93(\mathrm{~s}, 2 \mathrm{H}), 5.54(\mathrm{~d}, J=9.7 \mathrm{~Hz}, 1 \mathrm{H}), 3.98$ 
(s, 1H), 1.69 (s, 3H). ${ }^{13} \mathrm{C}$ NMR (101 MHz, Chloroform- $d$ ) $\delta$ 147.9, 146.4, 143.6, 142.5, 129.9, 129.1, 127.0, 123.4, 119.1, 118.4, 117.4, 112.5, 107.9, 106.9, 101.2, 57.3, 30.5. HRMS (ESI) m/z: $[\mathrm{M}+\mathrm{H}]^{+}$Calcd for $\mathrm{C}_{17} \mathrm{H}_{16} \mathrm{NO}_{2}{ }^{+}$266.1176; Found 266.1172 .

2-methyl-2-(thiophen-3-yl)-1,2-dihydroquinoline (1j)<smiles>CC1(c2ccsc2)C=Cc2ccccc2N1</smiles>

Green oil, $107 \mathrm{mg}$, 47\% yield from S1 $(210 \mathrm{mg}$ ), purified by column chromatography (petroleum ether:DCM = 6:1). ${ }^{1} \mathrm{H}$ NMR $(400 \mathrm{MHz}$, Chloroform- $d$ ) $\delta 7.27(\mathrm{~d}, J=3.2 \mathrm{~Hz}, 1 \mathrm{H})$, $7.16(\mathrm{ddd}, J=13.3,4.0,1.4 \mathrm{~Hz}, 2 \mathrm{H}), 6.99(\mathrm{td}, J=7.7,1.6 \mathrm{~Hz}, 1 \mathrm{H}), 6.92(\mathrm{dd}, J=7.4,1.6 \mathrm{~Hz}$, $1 \mathrm{H}), 6.67-6.56(\mathrm{~m}, 1 \mathrm{H}), 6.42(\mathrm{~d}, J=7.9 \mathrm{~Hz}, 1 \mathrm{H}), 6.34(\mathrm{~d}, J=9.7 \mathrm{~Hz}, 1 \mathrm{H}), 5.57(\mathrm{~d}, J=9.7 \mathrm{~Hz}$, 1H), 4.04 (s, 1H), 1.72 (s, 3H) ${ }^{13} \mathrm{C}$ NMR (101 MHz, Chloroform- $d$ ) $\delta$ 150.7, 142.6, 129.5, 129.0, 127.0, 126.4, 126.2, 123.6, 119.5, 119.4, 117.5, 112.6, 55.6, 30.5. HRMS (ESI) m/z: $[\mathrm{M}+\mathrm{H}]^{+}$Calcd for $\mathrm{C}_{14} \mathrm{H}_{14} \mathrm{NS}^{+} 228.0841$; Found 228.0837.

2-(furan-3-yl)-2-methyl-1,2-dihydroquinoline (1k)<smiles>CC1(c2ccoc2)C=Cc2ccccc2N1</smiles>

White oil, $104 \mathrm{mg}$, 49\% yield from S1 (196 mg), purified by column chromatography (petroleum ether:DCM = 6:1). ${ }^{1} \mathrm{H}$ NMR $(400 \mathrm{MHz}$, Chloroform- $d$ ) $\delta 7.32(\mathrm{dt}, J=16.3,1.5 \mathrm{~Hz}$, 2H), $6.98(\mathrm{td}, J=7.6,1.5 \mathrm{~Hz}, 1 \mathrm{H}), 6.92(\mathrm{dd}, J=7.5,1.5 \mathrm{~Hz}, 1 \mathrm{H}), 6.61(\mathrm{td}, J=7.4,1.1 \mathrm{~Hz}$, 1H), $6.45-6.38(\mathrm{~m}, 2 \mathrm{H}), 6.35(\mathrm{~d}, J=9.7 \mathrm{~Hz}, 1 \mathrm{H}), 5.54(\mathrm{~d}, J=9.7 \mathrm{~Hz}, 1 \mathrm{H}), 3.94(\mathrm{~s}, 1 \mathrm{H}), 1.63$ (s, 3H) ${ }^{13} \mathrm{C}$ NMR (101 MHz, Chloroform- $d$ ) $\delta 143.5,142.7,137.9,134.2,129.3$, 129.0, 126.9, 124.0, 119.6, 117.7, 112.8, 109.0, 52.6, 29.9. HRMS (ESI) m/z: $[\mathrm{M}+\mathrm{H}]^{+}$Calcd for $\mathrm{C}_{14} \mathrm{H}_{14} \mathrm{NO}^{+}$ 212.1070; Found 212.1067. 
2-ethyl-2-phenyl-1,2-dihydroquinoline (11)<smiles>CCC1(c2ccccc2)C=Cc2ccccc2N1</smiles>

Yellow oil, $94 \mathrm{mg}$, $40 \%$ yield from S1 (205 mg), purified by column chromatography (petroleum ether:DCM = 6:1). ${ }^{1} \mathrm{H}$ NMR $(400 \mathrm{MHz}$, Chloroform- $d$ ) $\delta 7.46(\mathrm{~d}, J=7.8 \mathrm{~Hz}, 2 \mathrm{H})$, $7.34(\mathrm{t}, J=7.6 \mathrm{~Hz}, 2 \mathrm{H}), 7.22(\mathrm{t}, J=7.3 \mathrm{~Hz}, 1 \mathrm{H}), 7.03-6.95(\mathrm{~m}, 1 \mathrm{H}), 6.91-6.76(\mathrm{~m}, 1 \mathrm{H})$, $6.57(\mathrm{t}, J=7.4 \mathrm{~Hz}, 1 \mathrm{H}), 6.50(\mathrm{~d}, J=7.9 \mathrm{~Hz}, 1 \mathrm{H}), 6.39(\mathrm{~d}, J=9.8 \mathrm{~Hz}, 1 \mathrm{H}), 5.57(\mathrm{~d}, J=9.8 \mathrm{~Hz}$, 1H), $4.03(\mathrm{~s}, 1 \mathrm{H}), 2.09(\mathrm{dq}, J=14.6,7.4 \mathrm{~Hz}, 1 \mathrm{H}), 1.90(\mathrm{dq}, J=14.5,7.4 \mathrm{~Hz}, 1 \mathrm{H}), 1.00(\mathrm{t}, J=$ 7.4 Hz, 3H). ${ }^{13} \mathrm{C}$ NMR (101 MHz, Chloroform- $d$ ) $\delta$ 148.7, 143.4, 129.0, 128.6, 128.4, 127.0, 126.7, 125.4, 124.6, 119.3, 117.0, 112.2, 60.9, 35.6, 8.8. HRMS (ESI) m/z: $[\mathrm{M}+\mathrm{H}]^{+}$Calcd for $\mathrm{C}_{17} \mathrm{H}_{18} \mathrm{~N}^{+} 236.1434$; Found 236.1431.

2-ethyl-2-(4-methoxyphenyl)-1,2-dihydroquinoline (1 m)<smiles>CCC1(c2ccc(OC)cc2)C=Cc2ccccc2N1</smiles>

Yellow oil, $114 \mathrm{mg}, 43 \%$ yield from S1 (235 mg), purified by column chromatography (petroleum ether:DCM = 6:1). ${ }^{1} \mathrm{H}$ NMR $(500 \mathrm{MHz}$, Chloroform- $d) \delta 7.38(\mathrm{~d}, J=8.8 \mathrm{~Hz}, 2 \mathrm{H})$, $6.99(\mathrm{td}, J=7.6,1.6 \mathrm{~Hz}, 1 \mathrm{H}), 6.91-6.81(\mathrm{~m}, 3 \mathrm{H}), 6.56(\mathrm{td}, J=7.3,1.1 \mathrm{~Hz}, 1 \mathrm{H}), 6.47(\mathrm{~d}, J=$ $7.9 \mathrm{~Hz}, 1 \mathrm{H}), 6.37$ (d, $J=9.8 \mathrm{~Hz}, 1 \mathrm{H}), 5.52$ (d, $J=9.8 \mathrm{~Hz}, 1 \mathrm{H}), 3.97$ (s, 1H), 3.79 (s, 3H), 2.05 $(\mathrm{dq}, J=14.5,7.3 \mathrm{~Hz}, 1 \mathrm{H}), 1.88(\mathrm{dq}, J=14.5,7.4 \mathrm{~Hz}, 1 \mathrm{H}), 0.99(\mathrm{t}, J=7.4 \mathrm{~Hz}, 3 \mathrm{H}) .{ }^{13} \mathrm{C}$ NMR (126 MHz, Chloroform- $d$ ) $\delta 158.3,143.3,141.1,129.0,128.6,127.0,126.6,124.3,119.2$, 116.9, 113.8, 112.1, 60.4, 55.4, 35.5, 8.8. HRMS (ESI) m/z: $[\mathrm{M}+\mathrm{H}]^{+}$Calcd for $\mathrm{C}_{18} \mathrm{H}_{20} \mathrm{NO}^{+}$ 266.1539; Found 266.1536.

2-ethyl-2-(3-methoxyphenyl)-1,2-dihydroquinoline (1n) 


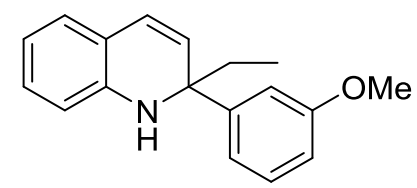

Yellow oil, $111 \mathrm{mg}, 42 \%$ yield from S1 (235 mg), purified by column chromatography (petroleum ether:DCM = 6:1). ${ }^{1} \mathrm{H}$ NMR $(500 \mathrm{MHz}$, Chloroform- $d$ ) $\delta 7.25(\mathrm{~d}, J=7.6 \mathrm{~Hz}, 1 \mathrm{H})$, $7.07-6.94(\mathrm{~m}, 3 \mathrm{H}), 6.87(\mathrm{dd}, J=7.4,1.5 \mathrm{~Hz}, 1 \mathrm{H}), 6.75(\mathrm{dd}, J=8.1,2.5 \mathrm{~Hz}, 1 \mathrm{H}), 6.56(\mathrm{td}, J$ $=7.3,1.1 \mathrm{~Hz}, 1 \mathrm{H}), 6.50(\mathrm{~d}, J=7.9 \mathrm{~Hz}, 1 \mathrm{H}), 6.38(\mathrm{~d}, J=9.8 \mathrm{~Hz}, 1 \mathrm{H}), 5.57(\mathrm{~d}, J=9.8 \mathrm{~Hz}, 1 \mathrm{H})$, $4.02(\mathrm{~s}, 1 \mathrm{H}), 3.78(\mathrm{~s}, 3 \mathrm{H}), 2.05(\mathrm{dt}, J=14.4,7.2 \mathrm{~Hz}, 1 \mathrm{H}), 1.88(\mathrm{dq}, J=14.6,7.4 \mathrm{~Hz}, 1 \mathrm{H}), 0.98$ $(\mathrm{t}, J=7.4 \mathrm{~Hz}, 3 \mathrm{H}) .{ }^{13} \mathrm{C}$ NMR $(126 \mathrm{MHz}$, Chloroform- $d) \delta 159.8,150.5,143.3,129.6,129.0$, 128.3, 127.0, 124.7, 119.3, 117.7, 117.1, 112.3, 112.0, 111.4, 60.9, 55.3, 35.6, 8.8. HRMS (ESI) m/z: $[\mathrm{M}+\mathrm{H}]^{+}$Calcd for $\mathrm{C}_{18} \mathrm{H}_{20} \mathrm{NO}^{+} 266.1539$; Found 266.1536 .

2-methyl-2-phenyl-1,2,3,4-tetrahydroquinoline (1o)<smiles>CC1(c2ccccc2)CCc2ccccc2N1</smiles>

Yellow oil, $216 \mathrm{mg}$, 97\% yield from 1a (221 mg), purified by column chromatography (petroleum ether:DCM = 6:1). ${ }^{1} \mathrm{H}$ NMR $(400 \mathrm{MHz}$, Chloroform- $d$ ) $\delta 7.48-7.38(\mathrm{~m}, 2 \mathrm{H}), 7.31$ (t, $J=7.7 \mathrm{~Hz}, 2 \mathrm{H}), 7.25-7.18(\mathrm{~m}, 1 \mathrm{H}), 7.10-7.00(\mathrm{~m}, 1 \mathrm{H}), 6.92(\mathrm{~d}, J=7.4 \mathrm{~Hz}, 1 \mathrm{H}), 6.70-$ $6.57(\mathrm{~m}, 2 \mathrm{H}), 4.13(\mathrm{~s}, 1 \mathrm{H}), 2.62(\mathrm{dt}, J=16.3,4.8 \mathrm{~Hz}, 1 \mathrm{H}), 2.34(\mathrm{ddd}, J=16.4,11.2,5.2 \mathrm{~Hz}$, $1 \mathrm{H}), 2.22(\mathrm{dt}, J=12.9,4.8 \mathrm{~Hz}, 1 \mathrm{H}), 1.93(\mathrm{ddd}, J=12.9,11.2,4.9 \mathrm{~Hz}, 1 \mathrm{H}), 1.59(\mathrm{~s}, 3 \mathrm{H}) .{ }^{13} \mathrm{C}$ NMR (101 MHz, Chloroform-d) $\delta 148.3,143.9,129.3,128.4,127.0,126.4,125.5,120.5$, 116.7, 113.5, 55.5, 35.4, 30.7, 24.4. HRMS (ESI) m/z: $[\mathrm{M}+\mathrm{H}]^{+}$Calcd for $\mathrm{C}_{16} \mathrm{H}_{18} \mathrm{~N}^{+}$224.1434; Found 224.1432.

2,3-dimethyl-2-phenyl-1,2-dihydroquinoline (1p)<smiles>CC1=Cc2ccccc2NC1(C)c1ccccc1</smiles> 
Yellow solid, $105 \mathrm{mg}$, 48\% yield from S1 (219 mg), purified by column chromatography (petroleum ether:DCM = 5:1). ${ }^{1} \mathrm{H}$ NMR $(400 \mathrm{MHz}$, Chloroform- $d$ ) $\delta 7.65-7.54(\mathrm{~m}, 2 \mathrm{H}), 7.33$ (t, $J=7.5 \mathrm{~Hz}, 2 \mathrm{H}), 7.24$ (d, $J=7.3 \mathrm{~Hz}, 1 \mathrm{H}), 6.98-6.90(\mathrm{~m}, 1 \mathrm{H}), 6.91-6.81(\mathrm{~m}, 1 \mathrm{H}), 6.59$ (t, $J=7.4 \mathrm{~Hz}, 1 \mathrm{H}), 6.35(\mathrm{~d}, J=7.8 \mathrm{~Hz}, 1 \mathrm{H}), 6.21(\mathrm{~s}, 1 \mathrm{H}), 3.86(\mathrm{~s}, 1 \mathrm{H}), 1.76(\mathrm{~s}, 3 \mathrm{H}), 1.62-1.56$ $(\mathrm{m}, 3 \mathrm{H}) .{ }^{13} \mathrm{C}$ NMR (101 MHz, Chloroform- $d$ ) $\delta$ 147.3, 142.0, 136.3, 128.3, 128.1, 127.2, 126.6, 125.9, 122.0, 120.3, 117.4, 112.0, 60.8, 26.3, 19.7. HRMS (ESI) m/z: $[\mathrm{M}+\mathrm{H}]^{+}$Calcd for $\mathrm{C}_{17} \mathrm{H}_{18} \mathrm{~N}^{+} 236.1434$; Found 236.1431.

2-(3,5-dimethylphenyl)-2-methyl-1,2-dihydroquinoline (1q)<smiles>Cc1cc(C)cc(C2(C)C=Cc3ccccc3N2)c1</smiles>

White solid, $116 \mathrm{mg}, 50 \%$ yield from S1 (233 mg), purified by column chromatography (petroleum ether:DCM = 6:1). ${ }^{1} \mathrm{H}$ NMR (400 MHz, Chloroform- $d$ ) $\delta 7.14(\mathrm{~s}, 2 \mathrm{H}), 7.00(\mathrm{t}, J=$ $7.7 \mathrm{~Hz}, 1 \mathrm{H}), 6.95-6.74(\mathrm{~m}, 2 \mathrm{H}), 6.59(\mathrm{t}, J=7.4 \mathrm{~Hz}, 1 \mathrm{H}), 6.45(\mathrm{~d}, J=7.9 \mathrm{~Hz}, 1 \mathrm{H}), 6.33(\mathrm{~d}, J$ $=9.7 \mathrm{~Hz}, 1 \mathrm{H}), 5.58(\mathrm{~d}, J=9.7 \mathrm{~Hz}, 1 \mathrm{H}), 4.00(\mathrm{~s}, 1 \mathrm{H}), 2.32(\mathrm{~s}, 6 \mathrm{H}), 1.70(\mathrm{~s}, 3 \mathrm{H}) .{ }^{13} \mathrm{C} \mathrm{NMR}$ (101 MHz, Chloroform- $d$ ) $\delta$ 149.2, 142.7, 138.0, 130.1, 129.0, 128.6, 127.0, 123.5, 123.3, 119.2, 117.2, 112.5, 57.4, 30.4, 21.7. HRMS (ESI) m/z: $[\mathrm{M}+\mathrm{H}]^{+}$Calcd for $\mathrm{C}_{18} \mathrm{H}_{20} \mathrm{~N}^{+} 250.1590$; Found 250.1586 .

\section{Kinetic resolution of DHQs through asymmetric bromination:}<smiles>[R]CC([R])([Al])Nc1ccccc1CC</smiles>

$( \pm)-1$

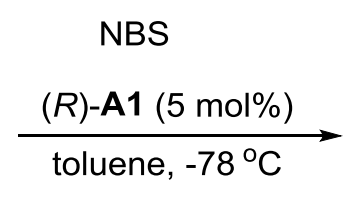

$24 \mathrm{~h}$

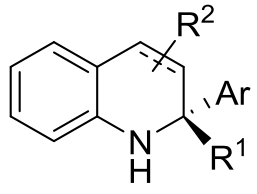

$(R)-1$<smiles>[R]CC1([Al])Nc2ccc(Br)cc2CC1[R]</smiles>

$(S)-2$

General procedure: In a $25 \mathrm{~mL}$ vial containing a magnetic stir bar, was added racemic $\mathbf{1}(0.2$ mmol) and CPA (R)-A1 (7.2 mg, $0.01 \mathrm{mmol}, 5 \mathrm{~mol} \%)$ under $\mathrm{N}_{2}$ atmosphere. After adding dry 
toluene $(5 \mathrm{~mL})$, the mixture was cooled to $-78^{\circ} \mathrm{C}$ and stirred for 30 minutes. Then a solution of NBS (21 mg, $0.12 \mathrm{mmol}, 0.6$ equiv.) in toluene $(5 \mathrm{~mL})$ was added dropwisely at this temperature. After stirring at $-78{ }^{\circ} \mathrm{C}$ for $12 \sim 24 \mathrm{~h}$, the reaction mixture was quenched with saturated $\mathrm{NaHSO}_{3}$ aqueous solution $(1 \mathrm{~mL})$, extracted with EtOAc for 3 times. The combined organic layer was washed with brine, dried over $\mathrm{Na}_{2} \mathrm{SO}_{4}$, filtered through Celite and concentrated in vacuo to give a residue, which was purified by column chromatography (petroleum ether:EtOAc = 20:1) to afford recovered $(R)-\mathbf{1}$ and $\mathbf{2}$.

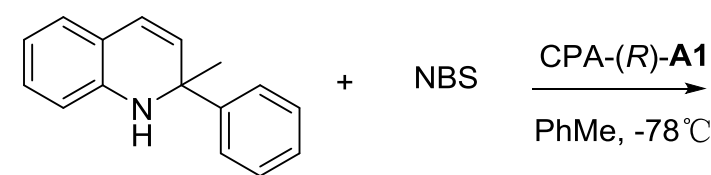

$( \pm)-1 \mathrm{a}$<smiles>CC1(c2ccccc2)C=Cc2ccccc2N1</smiles>

$R-1 \mathrm{a}$<smiles>C[C@]1(c2ccccc2)C=Cc2cc(Br)ccc2N1</smiles>

$S-2 a$

The reaction was performed on $0.2 \mathrm{mmol}$ scale with $(R)-\mathbf{A 1}(5 \mathrm{~mol} \%)$ as catalyst at $-78{ }^{\circ} \mathrm{C}$ for $24 \mathrm{~h}$ and then purified by column chromatography (petroleum ether:EtOAc $=20: 1$ ) to afford the products.

(R)-2-methyl-2-phenyl-1,2-dihydroquinoline $((R)$-1a)<smiles>C[C@]1(c2ccccc2)C=Cc2ccccc2N1</smiles>

Yellow oil, $17.6 \mathrm{mg}, 39 \%$. $[\alpha]_{\mathrm{D}}^{20}=+142.4$ (c 1.0, CHCl3). HPLC (Chiralpak IA, hexanes/isopropanol $=97 / 3$, flow rate $=1.0 \mathrm{~mL} / \mathrm{min}, \mathrm{I}=280 \mathrm{~nm}$ ) $t_{\mathrm{R}}=6.6 \mathrm{~min}$ (major), $7.3 \mathrm{~min}$ (minor), $93 \%$ ee.

(S)-6-bromo-2-methyl-2-phenyl-1,2-dihydroquinoline ((S)-2a)<smiles>C[C@]1(c2ccccc2)C=Cc2cc(Br)ccc2N1</smiles>

White solid, $25.5 \mathrm{mg}, 42 \% .{ }^{1} \mathrm{H}$ NMR (400 MHz, Chloroform- $d$ ) $\delta 7.51-7.44(\mathrm{~m}, 2 \mathrm{H}), 7.34(\mathrm{t}$, $J=7.7 \mathrm{~Hz}, 2 \mathrm{H}), 7.23(\mathrm{~d}, J=7.3 \mathrm{~Hz}, 1 \mathrm{H}), 7.05(\mathrm{dd}, J=8.4,2.3 \mathrm{~Hz}, 1 \mathrm{H}), 7.00(\mathrm{~d}, J=2.3 \mathrm{~Hz}$, $1 \mathrm{H}), 6.32(\mathrm{~d}, J=8.4 \mathrm{~Hz}, 1 \mathrm{H}), 6.26(\mathrm{~d}, J=9.8 \mathrm{~Hz}, 1 \mathrm{H}), 5.62(\mathrm{~d}, J=9.8 \mathrm{~Hz}, 1 \mathrm{H}), 4.05(\mathrm{~s}, 1 \mathrm{H})$, 
$1.72(\mathrm{~s}, 3 \mathrm{H}) .{ }^{13} \mathrm{C}$ NMR (101 MHz, Chloroform- $d$ ) $\delta$ 148.6, 141.6, 131.4, 131.1, 129.3, 128.6, 127.2, 125.4, 122.4, 121.1, 114.0, 108.6, 57.6, 30.2. $[\alpha]_{\mathrm{D}}^{20}=-162.6(\mathrm{c} 1.0, \mathrm{CHCl} 3)$. HPLC $($ Chiralpak IA, hexanes/isopropanol $=97 / 3$, flow rate $=1.0 \mathrm{~mL} / \mathrm{min}, \mathrm{I}=254 \mathrm{~nm}) \mathrm{t}_{\mathrm{R}}=8.9 \mathrm{~min}$ (minor), 9.7 min (major), 94\% ee. HRMS (ESI) m/z: $[\mathrm{M}+\mathrm{H}]^{+}$Calcd for $\mathrm{C}_{16} \mathrm{H}_{15} \mathrm{BrN}^{+} 300.0382$; Found 300.0380 .

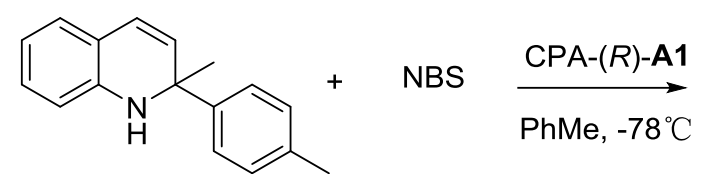

$( \pm)-1 \mathrm{~b}$<smiles>Cc1ccc(C2(C)C=Cc3ccccc3N2)cc1</smiles>

$R-1 \mathrm{~b}$

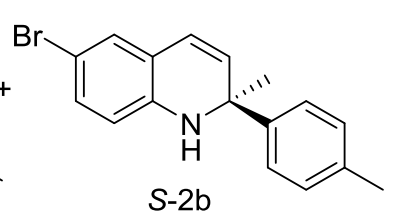

The reaction was performed on $0.2 \mathrm{mmol}$ scale with $(R)-\mathbf{A 1}(5 \mathrm{~mol} \%)$ as catalyst at $-78{ }^{\circ} \mathrm{C}$ for $24 \mathrm{~h}$ and then purified by column chromatography (petroleum ether:EtOAc $=20: 1$ ) to afford the products..

(R)-2-methyl-2-(p-tolyl)-1,2-dihydroquinoline $((R)-\mathbf{1 b})$

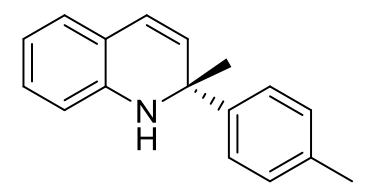

Yellow oil, $21.7 \mathrm{mg}, 46 \% .[\alpha]_{\mathrm{D}}^{20}=+213$ (c 1.0, $\mathrm{CHCl}_{3}$ ). HPLC (Chiralpak IA, hexanes/isopropanol $=97 / 3$, flow rate $=1.0 \mathrm{~mL} / \mathrm{min}, \mathrm{I}=254 \mathrm{~nm}$ ) $\mathrm{t}_{\mathrm{R}}=6.6 \mathrm{~min}$ (major), $7.5 \mathrm{~min}$ (minor), $93 \%$ ee.

(S)-6-bromo-2-methyl-2-(p-tolyl)-1,2-dihydroquinoline ((S)-2b)<smiles>Cc1ccc([C@]2(C)C=Cc3cc(Br)ccc3N2)cc1</smiles>

White solid, $30.1 \mathrm{mg}, 48 \% .{ }^{1} \mathrm{H}$ NMR (400 MHz, Chloroform- $d$ ) $\delta 7.37(\mathrm{~d}, J=7.9 \mathrm{~Hz}, 2 \mathrm{H})$, $7.15(\mathrm{~d}, J=7.8 \mathrm{~Hz}, 2 \mathrm{H}), 7.05(\mathrm{dd}, J=8.4,2.3 \mathrm{~Hz}, 1 \mathrm{H}), 7.00(\mathrm{~d}, J=2.3 \mathrm{~Hz}, 1 \mathrm{H}), 6.31(\mathrm{~d}, J=$ $8.4 \mathrm{~Hz}, 1 \mathrm{H}), 6.25(\mathrm{~d}, J=9.8 \mathrm{~Hz}, 1 \mathrm{H}), 5.61(\mathrm{~d}, J=9.8 \mathrm{~Hz}, 1 \mathrm{H}), 4.04(\mathrm{~s}, 1 \mathrm{H}), 2.33(\mathrm{~s}, 3 \mathrm{H}), 1.70$ (s, 3H). ${ }^{13} \mathrm{C}$ NMR (101 MHz, Chloroform- $d$ ) $\delta$ 145.8, 141.7, 136.9, 131.3, 131.2, 129.3, 129.2, $125.4,122.3,121.1,113.9,108.5,57.4,30.2,21.1 .[\alpha]_{\mathrm{D}}{ }^{20}=-82.4\left(\mathrm{c} 1.0, \mathrm{CHCl}_{3}\right)$. HPLC $($ Chiralpak IA, hexanes/isopropanol $=97 / 3$, flow rate $=1.0 \mathrm{~mL} / \mathrm{min}, \mathrm{I}=254 \mathrm{~nm}) \mathrm{t}_{\mathrm{R}}=8.6 \mathrm{~min}$ 
(minor), $9.1 \mathrm{~min}$ (major), 94\% ee. HRMS (ESI) m/z: $[\mathrm{M}+\mathrm{H}]^{+}$Calcd for $\mathrm{C}_{17} \mathrm{H}_{17} \mathrm{BrN}^{+} 314.0539$; Found 314.0536.

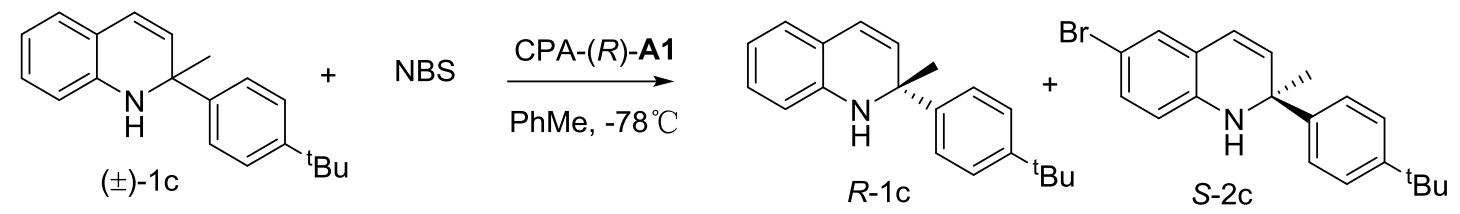

The reaction was performed on $0.2 \mathrm{mmol}$ scale with $(R)-\mathbf{A 1}(5 \mathrm{~mol} \%)$ as catalyst at $-78^{\circ} \mathrm{C}$ for $14 \mathrm{~h}$ and then purified by preparative TLC (petroleum ether:EtOAc $=10: 1$ ) to afford the products.

(R)-2-(4-(tert-butyl)phenyl)-2-methyl-1,2-dihydroquinoline $((R)-\mathbf{1 c})$<smiles>CC(C)(C)c1ccc([C@]2(C)C=Cc3ccccc3N2)cc1</smiles>

Green solid, $22.2 \mathrm{mg}, 40 \%$. $[\alpha]_{\mathrm{D}}^{20}=+221.0$ (c 1.0, $\mathrm{CHCl}_{3}$ ). HPLC (Chiralpak IA, hexanes/isopropanol $=97 / 3$, flow rate $=1.0 \mathrm{~mL} / \mathrm{min}, \mathrm{I}=254 \mathrm{~nm}$ ) $\mathrm{t}_{\mathrm{R}}=5.3 \mathrm{~min}$ (major), $5.8 \mathrm{~min}$ (minor), 98\% ee.

(S)-6-bromo-2-(4-(tert-butyl)phenyl)-2-methyl-1,2-dihydroquinoline ((S)-2c)<smiles>C[C@]1(c2ccc(Br)cc2)C=Cc2cc(Br)ccc2N1</smiles>

White solid, $27 \mathrm{mg}, 38 \% .{ }^{1} \mathrm{H}$ NMR (400 MHz, Chloroform- $d$ ) $\delta 7.54-7.30$ (m, 4H), $7.11-$ $6.88(\mathrm{~m}, 2 \mathrm{H}), 6.40-6.11(\mathrm{~m}, 2 \mathrm{H}), 5.62(\mathrm{~d}, J=9.7 \mathrm{~Hz}, 1 \mathrm{H}), 4.04(\mathrm{~s}, 1 \mathrm{H}), 1.71(\mathrm{~s}, 3 \mathrm{H}), 1.31(\mathrm{~s}$, 9H). ${ }^{13} \mathrm{C}$ NMR (101 MHz, Chloroform- $d$ ) $\delta 150.1,145.7,141.7,131.3,131.2,129.2,125.5$, $125.2,122.2,121.1,113.9,108.5,57.4,34.6,31.5,30.1 .[\alpha]_{\mathrm{D}}{ }^{20}=-125.0\left(\mathrm{c} 1.0, \mathrm{CHCl}_{3}\right) . \mathrm{HPLC}$ $($ Chiralpak IA, hexanes/isopropanol $=97 / 3$, flow rate $=1.0 \mathrm{~mL} / \mathrm{min}, \mathrm{I}=280 \mathrm{~nm}) \mathrm{t}_{\mathrm{R}}=7.1 \mathrm{~min}$ (minor), 7.4 min (major), 88\% ee. HRMS (ESI) m/z: $[\mathrm{M}+\mathrm{H}]^{+}$Calcd for $\mathrm{C}_{20} \mathrm{H}_{23} \mathrm{BrN}^{+} 356.1008$; Found 356.1006. 


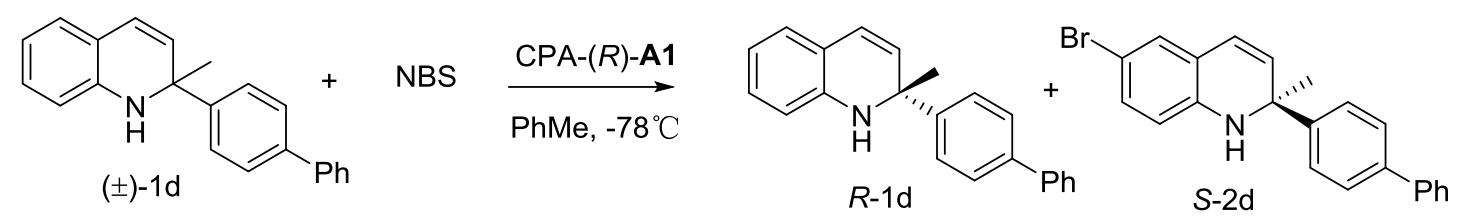

The reaction was performed on $0.2 \mathrm{mmol}$ scale with $(R)-\mathbf{A 1}(5 \mathrm{~mol} \%)$ as catalyst at $-78{ }^{\circ} \mathrm{C}$ for $12 \mathrm{~h}$ and then purified by column chromatography (petroleum ether:EtOAc $=20: 1$ ) to afford the products.

(R)-2-([1,1'-biphenyl]-4-yl)-2-methyl-1,2-dihydroquinoline $((R)-1 \mathbf{d})$

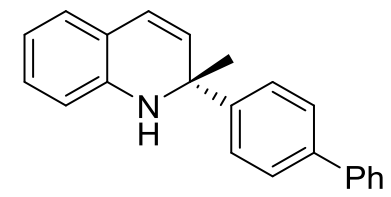

Green solid, $24.6 \mathrm{mg}, 41 \% .[\alpha]_{\mathrm{D}}^{20}=+70.4\left(\mathrm{c} 1.0, \mathrm{CHCl}_{3}\right)$. HPLC (Chiralpak IA, hexanes/isopropanol $=97 / 3$, flow rate $=1.0 \mathrm{~mL} / \mathrm{min}, \mathrm{I}=254 \mathrm{~nm}) \mathrm{t}_{\mathrm{R}}=11.4 \mathrm{~min}(\operatorname{minor}), 12.3$ $\min$ (major), $96 \%$ ee.

(S)-2-([1,1'-biphenyl]-4-yl)-6-bromo-2-methyl-1,2-dihydroquinoline ((S)-2d)<smiles>C[C@]1(c2ccc(-c3ccccc3)cc2)C=Cc2cc(Br)ccc2N1</smiles>

Yellow solid, $30.6 \mathrm{mg}, 41 \% .{ }^{1} \mathrm{H}$ NMR $(500 \mathrm{MHz}$, Chloroform- $d$ ) $\delta 7.57(\mathrm{dd}, J=7.9,1.9 \mathrm{~Hz}$, $6 \mathrm{H}), 7.44(\mathrm{t}, J=7.6 \mathrm{~Hz}, 2 \mathrm{H}), 7.38-7.30(\mathrm{~m}, 1 \mathrm{H}), 7.08(\mathrm{dd}, J=8.4,2.3 \mathrm{~Hz}, 1 \mathrm{H}), 7.03(\mathrm{~d}, J=$ $2.3 \mathrm{~Hz}, 1 \mathrm{H}), 6.35(\mathrm{~d}, J=8.4 \mathrm{~Hz}, 1 \mathrm{H}), 6.29(\mathrm{~d}, J=9.7 \mathrm{~Hz}, 1 \mathrm{H}), 5.66(\mathrm{~d}, J=9.7 \mathrm{~Hz}, 1 \mathrm{H}), 4.09$ (s, 1H), 1.76 (s, 3H). ${ }^{13} \mathrm{C}$ NMR (126 MHz, Chloroform- $d$ ) $\delta$ 147.6, 141.6, 140.7, 140.1, 131.5, $131.0,129.3,128.9,127.5,127.4,127.2,125.9,122.5,121.1,114.0,108.7,57.5,30.2 .[\alpha]_{D}{ }^{20}=$ -138.4 (c 1.0, $\left.\mathrm{CHCl}_{3}\right)$. HPLC (Chiralpak IB, hexanes/isopropanol $=97 / 3$, flow rate $=1.0$ $\mathrm{mL} / \mathrm{min}, \mathrm{I}=254 \mathrm{~nm}) \mathrm{t}_{\mathrm{R}}=16.1 \min ($ major), $17.4 \mathrm{~min}$ (minor), $78 \%$ ee. HRMS (ESI) m/z: $[\mathrm{M}+\mathrm{H}]^{+}$Calcd for $\mathrm{C}_{22} \mathrm{H}_{19} \mathrm{BrN}^{+}$376.0695; Found 376.0694. 


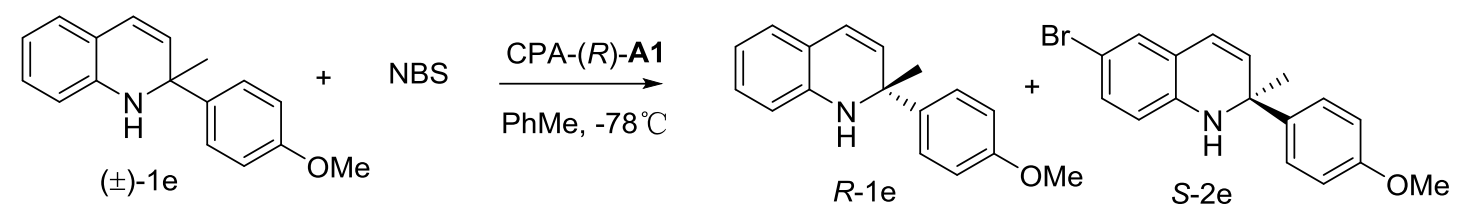

The reaction was performed on $0.2 \mathrm{mmol}$ scale with $(R)-\mathbf{A 1}(5 \mathrm{~mol} \%)$ as catalyst at $-78{ }^{\circ} \mathrm{C}$ for $24 \mathrm{~h}$ and then purified by column chromatography (petroleum ether:EtOAc $=20: 1$ ) to afford the products.

(R)-2-(4-methoxyphenyl)-2-methyl-1,2-dihydroquinoline $((R)-\mathbf{1 e})$<smiles>COc1ccc([C@]2(C)C=Cc3ccccc3N2)cc1</smiles>

Yellow solid, $19.6 \mathrm{mg}, 40 \% .[\alpha]_{\mathrm{D}}^{20}=+204.6$ (c 1.0, $\left.\mathrm{CHCl}_{3}\right)$. HPLC (Chiralpak IA, hexanes/isopropanol $=97 / 3$, flow rate $=1.0 \mathrm{~mL} / \mathrm{min}, \mathrm{I}=254 \mathrm{~nm}) \mathrm{t}_{\mathrm{R}}=9.9 \min (\operatorname{minor}), 11.4$ $\min$ (major), $98 \%$ ee.

(S)-6-bromo-2-(4-methoxyphenyl)-2-methyl-1,2-dihydroquinoline $((S)-2 \mathbf{e})$<smiles>COc1ccc(C2(C)C=Cc3cc(Br)ccc3N2)cc1</smiles>

Yellow solid, $26.3 \mathrm{mg}, 40 \% .{ }^{1} \mathrm{H}$ NMR (500 MHz, Chloroform- $d$ ) $\delta 7.42-7.39$ (m, 2H), 7.05 $(\mathrm{dd}, J=8.4,2.3 \mathrm{~Hz}, 1 \mathrm{H}), 7.01(\mathrm{~d}, J=2.3 \mathrm{~Hz}, 1 \mathrm{H}), 6.89-6.85(\mathrm{~m}, 2 \mathrm{H}), 6.30(\mathrm{~d}, J=8.4 \mathrm{~Hz}$, 1H), $6.25(\mathrm{~d}, J=9.7 \mathrm{~Hz}, 1 \mathrm{H}), 5.59(\mathrm{~d}, J=9.8 \mathrm{~Hz}, 1 \mathrm{H}), 3.79(\mathrm{~s}, 3 \mathrm{H}), 1.70(\mathrm{~s}, 3 \mathrm{H}) .{ }^{13} \mathrm{C}$ NMR (126 MHz, Chloroform-d) $\delta 158.6,141.5,140.9,131.2,131.2,129.1,126.6,122.0,120.9$, 113.8, 113.7, 108.4, 57.0, 55.3, 30.0. $[\alpha]_{\mathrm{D}}^{20}=-137.2\left(\mathrm{c} 1.0, \mathrm{CHCl}_{3}\right)$. HPLC $($ Chiralpak IB, hexanes/isopropanol $=97 / 3$, flow rate $=1.0 \mathrm{~mL} / \mathrm{min}, \mathrm{I}=254 \mathrm{~nm}$ ) $\mathrm{t}_{\mathrm{R}}=16.1 \mathrm{~min}($ major$), 17.4$ min (minor), $78 \%$ ee. HRMS (ESI) m/z: $[\mathrm{M}+\mathrm{H}]^{+}$Calcd for $\mathrm{C}_{17} \mathrm{H}_{17} \mathrm{BrNO}^{+} 330.0488$; Found 330.0488. 
<smiles>Cc1cccc(C2(C)C=Cc3ccccc3N2)c1</smiles>

$( \pm)-1 f$

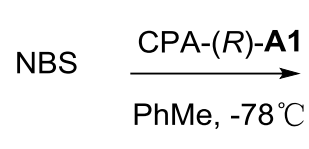

PhMe, $-78^{\circ} \mathrm{C}$<smiles>Cc1cccc([C@]2(C)C=Cc3ccccc3N2)c1</smiles>

$R-1 f$<smiles>Cc1cccc([C@]2(C)C=Cc3cc(Br)ccc3N2)c1</smiles>

The reaction was performed on $0.2 \mathrm{mmol}$ scale with $(R)-\mathbf{A 1}(5 \mathrm{~mol} \%)$ as catalyst at $-78{ }^{\circ} \mathrm{C}$ for $24 \mathrm{~h}$ and then purified by preparative TLC (petroleum ether:EtOAc $=10: 1$ ) to afford the products.

$(R)$-2-methyl-2-(m-tolyl)-1,2-dihydroquinoline ((R)-1f)<smiles>Cc1cccc([C@]2(C)C=Cc3ccccc3N2)c1</smiles>

White solid, $18.8 \mathrm{mg}, 40 \% .[\alpha]_{\mathrm{D}}^{20}=+168.2\left(\mathrm{c} 1.0, \mathrm{CHCl}_{3}\right)$. HPLC (Chiralpak IB, hexanes/isopropanol $=97 / 3$, flow rate $=1.0 \mathrm{~mL} / \mathrm{min}, \mathrm{I}=254 \mathrm{~nm}) \mathrm{t}_{\mathrm{R}}=6.3 \mathrm{~min}($ minor $), 6.6$ $\min$ (major), $98 \%$ ee.

(S)-6-bromo-2-methyl-2-(m-tolyl)-1,2-dihydroquinoline ((S)-2f)<smiles>Cc1cccc([C@]2(C)C=Cc3cc(Br)ccc3N2)c1</smiles>

White solid, $32 \mathrm{mg}, 47 \% .{ }^{1} \mathrm{H}$ NMR (400 MHz, Chloroform- $d$ ) $\delta 7.33-7.26(\mathrm{~m}, 2 \mathrm{H}), 7.23(\mathrm{~d}$, $J=7.6 \mathrm{~Hz}, 1 \mathrm{H}), 7.06(\mathrm{dt}, J=8.4,2.1 \mathrm{~Hz}, 2 \mathrm{H}), 7.01(\mathrm{~d}, J=2.3 \mathrm{~Hz}, 1 \mathrm{H}), 6.33(\mathrm{~d}, J=8.4 \mathrm{~Hz}$, $1 \mathrm{H}), 6.26(\mathrm{~d}, J=9.7 \mathrm{~Hz}, 1 \mathrm{H}), 5.62(\mathrm{~d}, J=9.8 \mathrm{~Hz}, 1 \mathrm{H}), 2.36(\mathrm{~s}, 3 \mathrm{H}), 1.71(\mathrm{~s}, 3 \mathrm{H}) .{ }^{13} \mathrm{C} \mathrm{NMR}$ (126 MHz, Chloroform-d) $\delta 148.5,141.5,138.1,131.3,131.0,129.1,128.4,127.8,126.2$, $122.3,122.2,120.9,113.9,108.4,57.5,30.1,21.7 .[\alpha]_{\mathrm{D}}{ }^{20}=-107.8\left(\mathrm{c} 1.0, \mathrm{CHCl}_{3}\right)$. HPLC $($ Chiralpak IA, hexanes/isopropanol $=97 / 3$, flow rate $=1.0 \mathrm{~mL} / \mathrm{min}, \mathrm{I}=254 \mathrm{~nm}) \mathrm{t}_{\mathrm{R}}=8.1 \mathrm{~min}$ (minor), 17.4 min (major), $93 \%$ ee. HRMS (ESI) m/z: $[\mathrm{M}+\mathrm{H}]^{+}$Calcd for $\mathrm{C}_{17} \mathrm{H}_{17} \mathrm{BrN}^{+} 314.0539$; Found 314.0536 . 


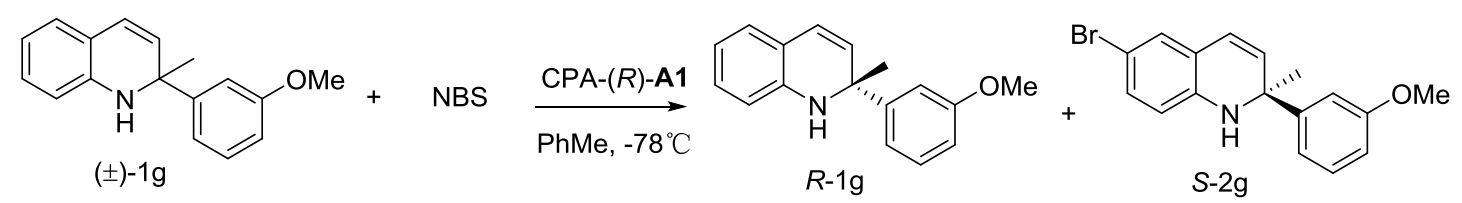

The reaction was performed on $0.2 \mathrm{mmol}$ scale with $(R)-\mathbf{A 1}(5 \mathrm{~mol} \%)$ as catalyst at $-78{ }^{\circ} \mathrm{C}$ for $22 \mathrm{~h}$ and then purified by column chromatography (petroleum ether:EtOAc $=10: 1$ ) to afford the products.

$(R)$-2-(3-methoxyphenyl)-2-methyl-1,2-dihydroquinoline $((R)-\mathbf{1 g})$<smiles>COc1cccc([C@]2(C)C=Cc3ccccc3N2)c1</smiles>

White solid, $17.2 \mathrm{mg}, 35 \%$. $[\alpha]_{\mathrm{D}}^{20}=+322.2\left(\mathrm{c} 1.0, \mathrm{CHCl}_{3}\right.$ ). HPLC (Chiralpak IA, hexanes/isopropanol $=97 / 3$, flow rate $=1.0 \mathrm{~mL} / \mathrm{min}, \mathrm{I}=254 \mathrm{~nm}$ ) $\mathrm{t}_{\mathrm{R}}=10.0 \mathrm{~min}($ minor), 10.5 $\min$ (major), $98 \%$ ee.

(S)-6-bromo-2-(3-methoxyphenyl)-2-methyl-1,2-dihydroquinoline ((S)-2g)<smiles>COc1cccc([C@]2(C)C=Cc3cc(Br)ccc3N2)c1</smiles>

White solid, $26.1 \mathrm{mg}, 40 \% .{ }^{1} \mathrm{H}$ NMR (500 MHz, Chloroform- $d$ ) $\delta 7.26$ (s, 1H), $7.09-7.02$ (m, 3H), $7.00(\mathrm{~s}, 1 \mathrm{H}), 6.78(\mathrm{~d}, J=8.1 \mathrm{~Hz}, 1 \mathrm{H}), 6.33(\mathrm{~d}, J=8.4 \mathrm{~Hz}, 1 \mathrm{H}), 6.26(\mathrm{~d}, J=9.8 \mathrm{~Hz}, 1 \mathrm{H})$, $5.62(\mathrm{~d}, J=9.7 \mathrm{~Hz}, 1 \mathrm{H}), 3.79(\mathrm{~s}, 3 \mathrm{H}), 1.70(\mathrm{~s}, 3 \mathrm{H}) .{ }^{13} \mathrm{C}$ NMR (126 MHz, Chloroform- $d$ ) $\delta$ $159.8,150.3,141.6,131.4,130.9,129.7,129.3,122.5,121.1,117.7,114.0,112.0,111.9$, 108.6, 57.6, 55.4, 30.2. $[\alpha]_{\mathrm{D}}^{20}=-107.6$ (c 1.0, $\left.\mathrm{CHCl}_{3}\right)$. HPLC (Chiralpak ID, hexanes/isopropanol $=97 / 3$, flow rate $=1.0 \mathrm{~mL} / \mathrm{min}, \mathrm{I}=254 \mathrm{~nm}$ ) $\mathrm{t}_{\mathrm{R}}=7.5 \mathrm{~min}$ (major), $8.4 \mathrm{~min}$ (minor), 94\% ee. HRMS (ESI) m/z: $[\mathrm{M}+\mathrm{H}]^{+}$Calcd for $\mathrm{C}_{17} \mathrm{H}_{17} \mathrm{BrNO}^{+}$330.0488; Found 330.0489 . 


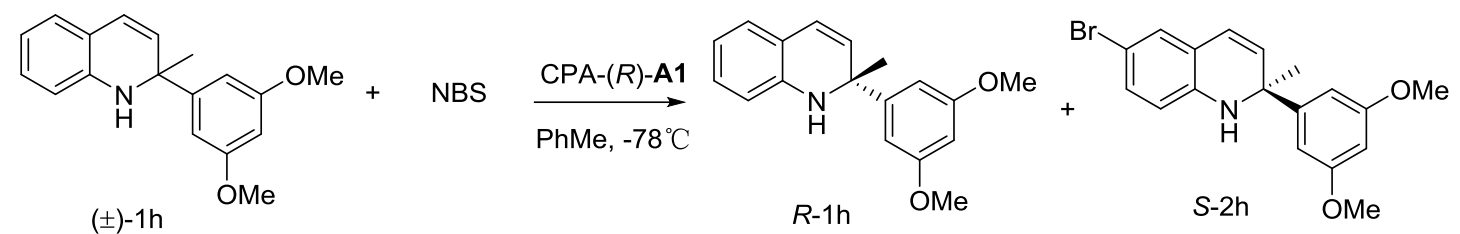

The reaction was performed on $0.2 \mathrm{mmol}$ scale with $(R)-\mathrm{A} 1(5 \mathrm{~mol} \%)$ as catalyst at $-78^{\circ} \mathrm{C}$ for $18 \mathrm{~h}$ and then purified by column chromatography (petroleum ether:EtOAc $=7: 1$ ) to afford the products.

(R)-2-(3,5-dimethoxyphenyl)-2-methyl-1,2-dihydroquinoline $((R)-\mathbf{1 h})$<smiles>COc1cc(OC)cc([C@]2(C)C=Cc3ccccc3N2)c1</smiles>

White solid, $16.2 \mathrm{mg}, 40 \% .[\alpha]_{\mathrm{D}}^{20}=+36.2\left(\mathrm{c} 1.0, \mathrm{CHCl}_{3}\right)$. HPLC (Chiralpak ID, hexanes/isopropanol $=97 / 3$, flow rate $=1.0 \mathrm{~mL} / \mathrm{min}, \mathrm{I}=254 \mathrm{~nm}) \mathrm{t}_{\mathrm{R}}=10.1 \mathrm{~min}(\operatorname{minor}), 12.1$ $\min$ (major), $99 \%$ ee.

(S)-6-bromo-2-(3,5-dimethoxyphenyl)-2-methyl-1,2-dihydroquinoline ((S)-2h)<smiles>COc1cc(OC)cc([C@]2(C)C=Cc3cc(Br)ccc3N2)c1</smiles>

White solid, $29.5 \mathrm{mg}, 41 \% .{ }^{1} \mathrm{H}$ NMR (500 MHz, Chloroform- $d$ ) $\delta 7.05(\mathrm{dd}, J=8.4,2.2 \mathrm{~Hz}$, 1H), $7.00(\mathrm{~d}, J=2.3 \mathrm{~Hz}, 1 \mathrm{H}), 6.69-6.53(\mathrm{~m}, 2 \mathrm{H}), 6.39-6.30(\mathrm{~m}, 2 \mathrm{H}), 6.26(\mathrm{~d}, J=9.7 \mathrm{~Hz}$, $1 \mathrm{H}), 5.61(\mathrm{~d}, J=9.7 \mathrm{~Hz}, 1 \mathrm{H}), 3.77(\mathrm{~s}, 6 \mathrm{H}), 1.67(\mathrm{~s}, 3 \mathrm{H}) .{ }^{13} \mathrm{C}$ NMR $(126 \mathrm{MHz}$, Chloroform- $d)$ $\delta 161.0,151.1,141.6,131.4,130.8,129.3,122.6,121.1,114.0,108.7,104.1,98.4,57.7,55.5$, 30.2. $[\alpha]_{\mathrm{D}}{ }^{20}=-14.2\left(\mathrm{c} 1.0, \mathrm{CHCl}_{3}\right)$. HPLC (Chiralpak IB, hexanes/isopropanol $=97 / 3$, flow rate $=1.0 \mathrm{~mL} / \mathrm{min}, \mathrm{I}=254 \mathrm{~nm}) \mathrm{t}_{\mathrm{R}}=13.7 \mathrm{~min}$ (minor), $14.7 \mathrm{~min}$ (major), $79 \%$ ee. HRMS (ESI) $\mathrm{m} / \mathrm{z}$ : $[\mathrm{M}+\mathrm{H}]^{+}$Calcd for $\mathrm{C}_{18} \mathrm{H}_{19} \mathrm{BrNO}_{2}{ }^{+}$360.0594; Found 360.0592 . 


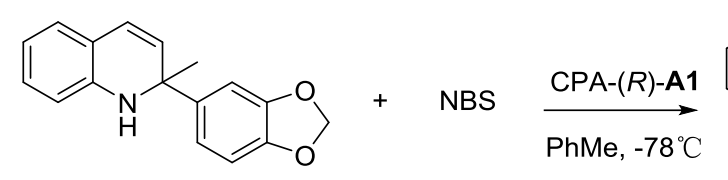

$( \pm)-1 \mathrm{i}$

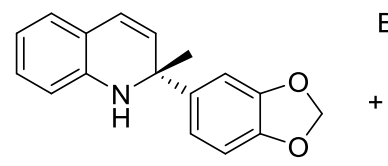

$R-1 \mathrm{i}$

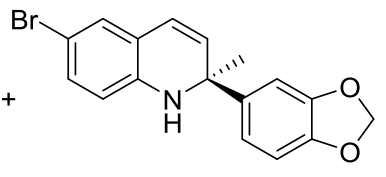

S-2i

The reaction was performed on $0.2 \mathrm{mmol}$ scale with $(R)-\mathbf{A 1}(5 \mathrm{~mol} \%)$ as catalyst at $-78{ }^{\circ} \mathrm{C}$ for $22 \mathrm{~h}$ and then purified by column chromatography (petroleum ether:EtOAc $=7: 1$ ) to afford products.

(R)-2-(benzo[d][1,3]dioxol-5-yl)-2-methyl-1,2-dihydroquinoline $((R)-1 \mathbf{i})$<smiles>C[C@]1(c2ccc3c(c2)OCO3)C=Cc2ccccc2N1</smiles>

Yellow solid, $22.2 \mathrm{mg}, 41 \% \cdot[\alpha]_{\mathrm{D}}^{20}=+208.2\left(\mathrm{c} 1.0, \mathrm{CHCl}_{3}\right)$. HPLC (Chiralpak IB, hexanes/isopropanol $=97 / 3$, flow rate $=1.0 \mathrm{~mL} / \mathrm{min}, \mathrm{I}=254 \mathrm{~nm}) \mathrm{t}_{\mathrm{R}}=10.8 \mathrm{~min}(\operatorname{minor}), 11.8$ $\min$ (major), $96 \%$ ee.

(S)-2-(benzo[d][1,3]dioxol-5-yl)-6-bromo-2-methyl-1,2-dihydroquinoline $((S)-2 \mathbf{i})$<smiles>C[C@]1(c2ccc3c(c2)OCO3)C=Cc2cc(Br)ccc2N1</smiles>

White solid, $31.4 \mathrm{mg}, 45 \% .{ }^{1} \mathrm{H}$ NMR (400 MHz, Chloroform- $d$ ) $\delta 7.08-6.97$ (m, 3H), 6.92 (dd, $J=8.2,1.9 \mathrm{~Hz}, 1 \mathrm{H}), 6.75(\mathrm{~d}, J=8.2 \mathrm{~Hz}, 1 \mathrm{H}), 6.34-6.07(\mathrm{~m}, 2 \mathrm{H}), 5.93(\mathrm{~s}, 2 \mathrm{H}), 5.57(\mathrm{~d}$, $J=9.8 \mathrm{~Hz}, 1 \mathrm{H}), 4.00(\mathrm{~s}, 1 \mathrm{H}), 1.67$ (s, 3H). ${ }^{13} \mathrm{C}$ NMR (101 MHz, Chloroform- $d$ ) $\delta$ 148.0, 146.6, 143.0, 141.5, 131.4, 131.0, 129.3, 122.3, 121.0, 118.4, 114.0, 108.6, 108.0, 106.8, 101.2, 57.4, 30.3. $[\alpha]_{\mathrm{D}}{ }^{20}=-96.0\left(\mathrm{c} 1.0, \mathrm{CHCl}_{3}\right)$. HPLC $($ Chiralpak IB, hexanes/isopropanol = $97 / 3$, flow rate $=1.0 \mathrm{~mL} / \mathrm{min}, \mathrm{I}=254 \mathrm{~nm}$ ) $\mathrm{t}_{\mathrm{R}}=14.5 \mathrm{~min}$ (major), $16.6 \mathrm{~min}$ (minor), $83 \%$ ee. HRMS (ESI) m/z: [M+H] $]^{+}$Calcd for $\mathrm{C}_{17} \mathrm{H}_{15} \mathrm{BrNO}_{2}{ }^{+}$344.0281; Found 344.0283. 


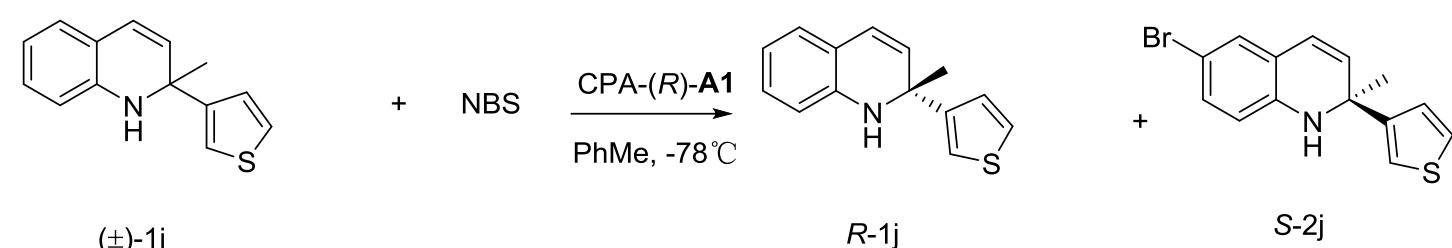

The reaction was performed on $0.2 \mathrm{mmol}$ scale with $(R)-\mathbf{A 1}(5 \mathrm{~mol} \%)$ as catalyst at $-78{ }^{\circ} \mathrm{C}$ for $16 \mathrm{~h}$ and then purified by column chromatography (petroleum ether:EtOAc $=15: 1$ ) to afford the products.

(R)-2-methyl-2-(thiophen-3-yl)-1,2-dihydroquinoline $((R)-\mathbf{1 j})$<smiles>C[C@]1(c2ccsc2)C=Cc2ccccc2N1</smiles>

Green oil, $19 \mathrm{mg}, 42 \% .[\alpha]_{\mathrm{D}}^{20}=+86.0$ (c 1.0, $\left.\mathrm{CHCl}_{3}\right)$. HPLC (Chiralpak IA, hexanes/isopropanol $=97 / 3$, flow rate $=1.0 \mathrm{~mL} / \mathrm{min}, \mathrm{I}=254 \mathrm{~nm}$ ) $\mathrm{t}_{\mathrm{R}}=7.4 \mathrm{~min}$ (major), $8.6 \mathrm{~min}$ (minor), $85 \%$ ee.

(S)-6-bromo-2-methyl-2-(thiophen-3-yl)-1,2-dihydroquinoline ((S)-2j)<smiles>C[C@]1(c2ccsc2)C=Cc2cc(Br)ccc2N1</smiles>

Green solid, $26 \mathrm{mg}, 44 \% .{ }^{1} \mathrm{H}$ NMR $(400 \mathrm{MHz}$, Chloroform- $d$ ) $\delta 7.28(\mathrm{~d}, J=3.3 \mathrm{~Hz}, 1 \mathrm{H}), 7.13$ $(\mathrm{dd}, J=7.2,3.9 \mathrm{~Hz}, 2 \mathrm{H}), 7.06-6.96(\mathrm{~m}, 2 \mathrm{H}), 6.28(\mathrm{dd}, J=14.4,9.1 \mathrm{~Hz}, 2 \mathrm{H}), 5.60(\mathrm{~d}, J=9.7$ $\mathrm{Hz}, 1 \mathrm{H}), 4.05$ (s, 1H), 1.71 (s, 3H). ${ }^{13} \mathrm{C}$ NMR (126 MHz, Chloroform- $d$ ) $\delta$ 150.2, 141.6, 131.4, $130.7,129.3,126.4,126.2,122.6,121.2,119.7,114.1,108.8,55.7,30.4 .[\alpha]_{D}^{20}=-70.8(\mathrm{c} 1.0$, $\left.\mathrm{CHCl}_{3}\right) . \mathrm{HPLC}($ Chiralpak IB, hexanes/isopropanol $=97 / 3$, flow rate $=1.0 \mathrm{~mL} / \mathrm{min}, \mathrm{I}=254$ $\mathrm{nm}) \mathrm{t}_{\mathrm{R}}=10.9 \min$ (minor), 11.6 min (major), $93 \%$ ee. HRMS (ESI) m/z: $[\mathrm{M}+\mathrm{H}]^{+}$Calcd for $\mathrm{C}_{14} \mathrm{H}_{13} \mathrm{BrNS}^{+}$305.9947; Found 305.9919. 


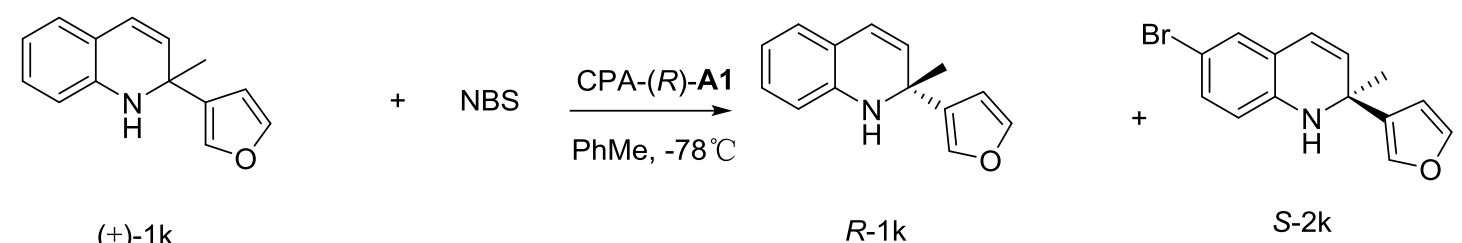

The reaction was performed on $0.2 \mathrm{mmol}$ scale with $(R)$-A1 $(5 \mathrm{~mol} \%)$ as catalyst at $-78{ }^{\circ} \mathrm{C}$ for $20 \mathrm{~h}$ and then purified by column chromatography (petroleum ether:EtOAc $=15: 1$ ) to afford the products.

(R)-2-(furan-3-yl)-2-methyl-1,2-dihydroquinoline $((R)-\mathbf{1 k})$<smiles>C[C@]1(c2ccoc2)C=Cc2ccccc2N1</smiles>

White oil, $20 \mathrm{mg}, 47 \% .[\alpha]_{\mathrm{D}}^{20}=+59.4$ (c 1.0, $\left.\mathrm{CHCl}_{3}\right)$. HPLC (Chiralpak IA, hexanes/isopropanol $=97 / 3$, flow rate $=1.0 \mathrm{~mL} / \mathrm{min}, \mathrm{I}=280 \mathrm{~nm}$ ) $\mathrm{t}_{\mathrm{R}}=7.2 \mathrm{~min}$ (major), $8.1 \mathrm{~min}$ (minor), $94 \%$ ee.

(S)-6-bromo-2-(furan-3-yl)-2-methyl-1,2-dihydroquinoline ((S)-2k)<smiles>C[C@]1(c2ccoc2)C=Cc2cc(Br)ccc2N1</smiles>

White solid, $30 \mathrm{mg}, 48 \% .{ }^{1} \mathrm{H}$ NMR (400 MHz, Chloroform- $d$ ) $\delta 7.39-7.27$ (m, 2H), $7.08-$ $6.99(\mathrm{~m}, 2 \mathrm{H}), 6.53-6.36(\mathrm{~m}, 1 \mathrm{H}), 6.29(\mathrm{t}, J=9.2 \mathrm{~Hz}, 2 \mathrm{H}), 5.57(\mathrm{~d}, J=9.7 \mathrm{~Hz}, 1 \mathrm{H}), 3.96(\mathrm{~s}$, 1H), 1.62 (s, 3H). ${ }^{13} \mathrm{C}$ NMR (101 MHz, Chloroform- $d$ ) $\delta$ 143.6, 141.6, 137.9, 133.8, 131.3, $130.4,129.2,122.9,121.4,114.3,109.0,108.8,52.7,29.8 .[\alpha]_{D}{ }^{20}=-27.6\left(\mathrm{c} 1.0, \mathrm{CHCl}_{3}\right)$. HPLC $($ Chiralpak IC, hexanes/isopropanol $=97 / 3$, flow rate $=1.0 \mathrm{~mL} / \mathrm{min}, \mathrm{I}=280 \mathrm{~nm}) \mathrm{t}_{\mathrm{R}}=$ 5.9 min (major), 7.4 min (minor), $79 \%$ ee. HRMS (ESI) m/z: $[\mathrm{M}+\mathrm{H}]^{+}$Calcd for $\mathrm{C}_{14} \mathrm{H}_{13} \mathrm{BrNO}^{+}$ 290.0175; Found 290.0174. 
<smiles>CCC1(c2ccccc2)C=Cc2ccccc2N1</smiles>

$( \pm)-11$

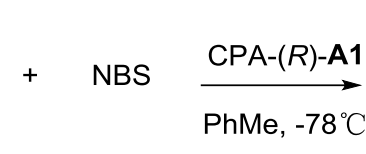

PhMe, $-78^{\circ} \mathrm{C}$

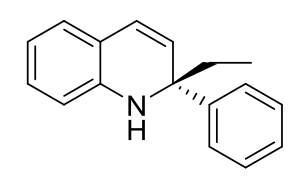

$R-11$

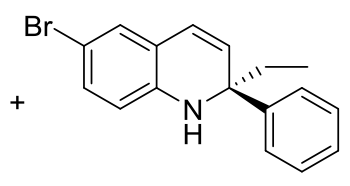

S-2I

The reaction was performed on $0.2 \mathrm{mmol}$ scale with $(R)-\mathbf{A 1}(5 \mathrm{~mol} \%)$ as catalyst at $-78{ }^{\circ} \mathrm{C}$ for $15 \mathrm{~h}$ and then purified by preparative TLC (petroleum ether:EtOAc $=10: 1$ ) to afford the products.

(R)-2-ethyl-2-phenyl-1,2-dihydroquinoline $((R)$-11)<smiles>CC[C@@]1(c2ccccc2)C=Cc2ccccc2N1</smiles>

Yellow oil, $19.7 \mathrm{mg}, 42 \% .[\alpha]_{\mathrm{D}}^{20}=+212.2\left(\mathrm{c} 1.0, \mathrm{CHCl}_{3}\right)$. HPLC (Chiralpak IA, hexanes/isopropanol $=97 / 3$, flow rate $=1.0 \mathrm{~mL} / \mathrm{min}, \mathrm{I}=254 \mathrm{~nm}$ ) $\mathrm{t}_{\mathrm{R}}=5.9 \mathrm{~min}$ (major), $6.4 \mathrm{~min}$ (minor), $82 \%$ ee.

(S)-6-bromo-2-ethyl-2-phenyl-1,2-dihydroquinoline ((S)-2l)<smiles>CC[C@@]1(c2ccccc2)C=Cc2cc(Br)ccc2N1</smiles>

White solid, $31.8 \mathrm{mg}, 48 \% .{ }^{1} \mathrm{H}$ NMR (400 MHz, Chloroform- $d$ ) $\delta 7.42(\mathrm{~d}, J=7.8 \mathrm{~Hz}, 2 \mathrm{H})$, 7.34 (t, $J=7.6 \mathrm{~Hz}, 2 \mathrm{H}), 7.23(\mathrm{t}, J=7.1 \mathrm{~Hz}, 1 \mathrm{H}), 7.06(\mathrm{dd}, J=8.4,2.3 \mathrm{~Hz}, 1 \mathrm{H}), 6.98(\mathrm{~d}, J=$ $2.3 \mathrm{~Hz}, 1 \mathrm{H}), 6.37(\mathrm{~d}, J=8.4 \mathrm{~Hz}, 1 \mathrm{H}), 6.31(\mathrm{~d}, J=9.8 \mathrm{~Hz}, 1 \mathrm{H}), 5.59(\mathrm{~d}, J=9.8 \mathrm{~Hz}, 1 \mathrm{H}), 4.02$ (s, 1H), $2.07(\mathrm{dq}, J=14.6,7.3 \mathrm{~Hz}, 1 \mathrm{H}), 1.89$ (dq, $J=14.6,7.4 \mathrm{~Hz}, 1 \mathrm{H}), 0.99$ (t, $J=7.4 \mathrm{~Hz}$, 3H). ${ }^{13} \mathrm{C}$ NMR (101 MHz, Chloroform- $d$ ) $\delta 148.3,142.3,131.4,129.5$, 129.3, 128.7, 127.0, $125.4,123.6,121.1,113.7,108.3,61.1,35.5,8.8 .[\alpha]_{\mathrm{D}}{ }^{20}=-117.8\left(\mathrm{c} 1.0, \mathrm{CHCl}_{3}\right) . \mathrm{HPLC}$ $($ Chiralpak IB, hexanes/isopropanol $=97 / 3$, flow rate $=1.0 \mathrm{~mL} / \mathrm{min}, \mathrm{I}=254 \mathrm{~nm}) \mathrm{t}_{\mathrm{R}}=9.6 \mathrm{~min}$ (major), $11.6 \mathrm{~min}$ (minor), 85\% ee. HRMS (ESI) m/z: $[\mathrm{M}+\mathrm{H}]^{+}$Calcd for $\mathrm{C}_{17} \mathrm{H}_{17} \mathrm{BrN}^{+} 314.0539$; Found 314.0539. 


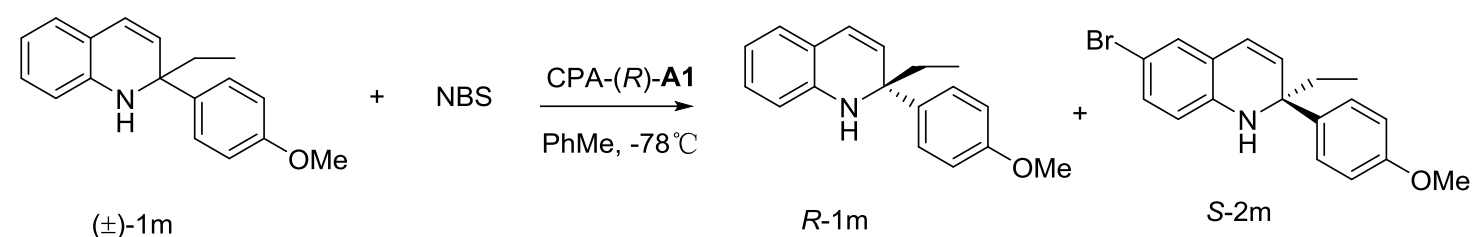

The reaction was performed on $0.2 \mathrm{mmol}$ scale with $(R)-\mathbf{A 1}(5 \mathrm{~mol} \%)$ as catalyst at $-78{ }^{\circ} \mathrm{C}$ for $13 \mathrm{~h}$ and then purified by column chromatography (petroleum ether:EtOAc $=10: 1$ ) to afford the products.

(R)-2-ethyl-2-(4-methoxyphenyl)-1,2-dihydroquinoline ((R)-1m)<smiles>CCC1(c2ccc(OC)cc2)C=Cc2ccccc2N1</smiles>

Yellow oil, $19.8 \mathrm{mg}, 38 \% .[\alpha]_{\mathrm{D}}^{20}=+321.8\left(\mathrm{c} 1.0, \mathrm{CHCl}_{3}\right)$. HPLC (Chiralpak IA, hexanes/isopropanol $=97 / 3$, flow rate $=1.0 \mathrm{~mL} / \mathrm{min}, \mathrm{I}=254 \mathrm{~nm}$ ) $t_{\mathrm{R}}=8.9 \mathrm{~min}$ (major), $9.8 \mathrm{~min}$ (minor), $86 \%$ ee.

(S)-6-bromo-2-ethyl-2-(4-methoxyphenyl)-1,2-dihydroquinoline ((S)-2m)<smiles>CC[C@](C)(c1ccc(OC)cc1)C1C=Cc2cc(Br)ccc2N1</smiles>

White solid, $27.9 \mathrm{mg}, 42 \% .{ }^{1} \mathrm{H}$ NMR (500 MHz, Chloroform- $d$ ) $\delta 7.39-7.31$ (m, 2H), 7.05 $(\mathrm{dd}, J=8.4,2.3 \mathrm{~Hz}, 1 \mathrm{H}), 6.98(\mathrm{~d}, J=2.2 \mathrm{~Hz}, 1 \mathrm{H}), 6.92-6.83(\mathrm{~m}, 2 \mathrm{H}), 6.34(\mathrm{~d}, J=8.5 \mathrm{~Hz}$, 1H), $6.30(\mathrm{~d}, J=9.8 \mathrm{~Hz}, 1 \mathrm{H}), 5.53(\mathrm{~d}, J=9.8 \mathrm{~Hz}, 1 \mathrm{H}), 3.97(\mathrm{~s}, 1 \mathrm{H}), 3.79(\mathrm{~s}, 3 \mathrm{H}), 2.03(\mathrm{dq}, J$ $=14.5,7.1 \mathrm{~Hz}, 1 \mathrm{H}), 1.88(\mathrm{dq}, J=14.5,7.4 \mathrm{~Hz}, 1 \mathrm{H}), 0.99(\mathrm{t}, J=7.4 \mathrm{~Hz}, 3 \mathrm{H}) .{ }^{13} \mathrm{C}$ NMR $(126$ MHz, Chloroform- $d$ ) $\delta 158.4,142.3,140.7,131.3,129.7,129.3,126.6,123.2,121.0,113.9$, 113.6, 108.1, 60.6, 55.4, 35.4, 8.8. $[\alpha]_{\mathrm{D}}{ }^{20}=-168.2\left(\mathrm{c} 1.0, \mathrm{CHCl}_{3}\right)$. HPLC (Chiralpak IA, hexanes/isopropanol $=97 / 3$, flow rate $=1.0 \mathrm{~mL} / \mathrm{min}, \mathrm{I}=280 \mathrm{~nm}) \mathrm{t}_{\mathrm{R}}=7.8 \mathrm{~min}($ minor), 8.3 
min (major), $83 \%$ ee. HRMS (ESI) m/z: $[\mathrm{M}+\mathrm{H}]^{+}$Calcd for $\mathrm{C}_{18} \mathrm{H}_{19} \mathrm{BrNO}^{+}$344.0645; Found 344.0644 .

OMe $+{ }_{N B S}$

$( \pm)-1 n$

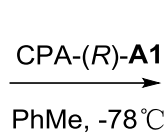

PhMe, $-78^{\circ} \mathrm{C}$

$R-1 n$

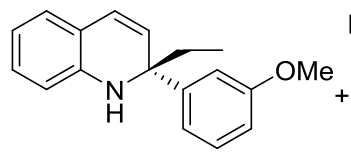

R-1n

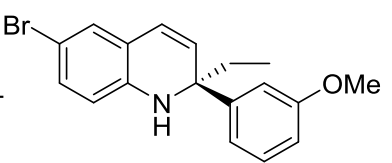

$S-2 n$

The reaction was performed on $0.2 \mathrm{mmol}$ scale with $(R)-\mathbf{A 1}(5 \mathrm{~mol} \%)$ as catalyst at $-78{ }^{\circ} \mathrm{C}$ for $13 \mathrm{~h}$ and then purified by column chromatography (petroleum ether:EtOAc $=10: 1$ ) to afford the products.

(R)-2-ethyl-2-(3-methoxyphenyl)-1,2-dihydroquinoline ((R)-1n)<smiles>CC[C@@]1(c2cccc(OC)c2)C=Cc2ccccc2N1</smiles>

Yellow oil, $25 \mathrm{mg}, 47 \% .[\alpha]_{\mathrm{D}}^{20}=+173.8\left(\mathrm{c} 1.0, \mathrm{CHCl}_{3}\right)$. HPLC (Chiralpak IB, hexanes/isopropanol $=97 / 3$, flow rate $=1.0 \mathrm{~mL} / \mathrm{min}, \mathrm{I}=254 \mathrm{~nm}) \mathrm{t}_{\mathrm{R}}=13.7 \mathrm{~min}(\operatorname{minor}), 15.2$ $\min$ (major), $89 \%$ ee.

(S)-6-bromo-2-ethyl-2-(3-methoxyphenyl)-1,2-dihydroquinoline ((S)-2n)<smiles>CC[C@](C)(c1cccc(OC)c1)C1C=Cc2cc(Br)ccc2N1</smiles>

White solid, $30 \mathrm{mg}, 43 \% .{ }^{1} \mathrm{H}$ NMR $(500 \mathrm{MHz}$, Chloroform- $d) \delta 7.25(\mathrm{~d}, J=5.8 \mathrm{~Hz}, 1 \mathrm{H}), 7.05$ (dd, $J=8.4,2.3 \mathrm{~Hz}, 1 \mathrm{H}), 7.03-6.93(\mathrm{~m}, 3 \mathrm{H}), 6.76(\mathrm{dd}, J=8.2,2.5 \mathrm{~Hz}, 1 \mathrm{H}), 6.37(\mathrm{~d}, J=8.4$ Hz, 1H), 6.31 (d, $J=9.8 \mathrm{~Hz}, 1 \mathrm{H}), 5.59$ (d, $J=9.8 \mathrm{~Hz}, 1 \mathrm{H}), 4.02(\mathrm{~s}, 1 \mathrm{H}), 3.78$ (s, 3H), 2.03 (dt, $J=14.6,7.3 \mathrm{~Hz}, 1 \mathrm{H}), 1.87(\mathrm{dq}, J=14.6,7.4 \mathrm{~Hz}, 1 \mathrm{H}), 0.97(\mathrm{~s}, 3 \mathrm{H}) .{ }^{13} \mathrm{C} \mathrm{NMR}(126 \mathrm{MHz}$, Chloroform- $d$ ) $\delta 159.8,150.0,142.3,131.4,129.7,129.4,129.3,123.6,121.1,117.6,113.7$, 112.1, 111.5, 108.3, 61.1, 55.3, 35.6, 8.8. $[\alpha]_{\mathrm{D}}{ }^{20}=-168.2\left(\mathrm{c} 1.0, \mathrm{CHCl}_{3}\right)$. HPLC (Chiralpak IC, hexanes/isopropanol $=97 / 3$, flow rate $=1.0 \mathrm{~mL} / \mathrm{min}, \mathrm{I}=280 \mathrm{~nm}) \mathrm{t}_{\mathrm{R}}=6.8 \mathrm{~min}($ minor $), 7.5$ 
min (major), $88 \%$ ee. HRMS (ESI) m/z: $[\mathrm{M}+\mathrm{H}]^{+}$Calcd for $\mathrm{C}_{18} \mathrm{H}_{19} \mathrm{BrNO}^{+} 344.0645$; Found 344.0644 .<smiles>CC1(c2ccccc2)CCc2ccccc2N1</smiles>

$( \pm)-10$

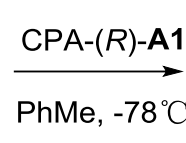

PhMe, $-78^{\circ} \mathrm{C}$

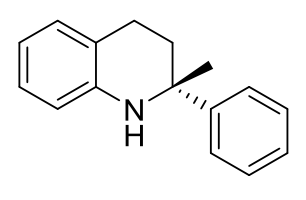

$R-10$

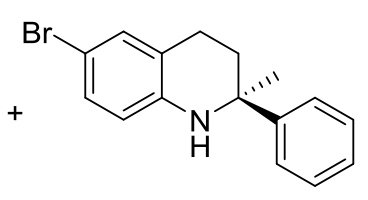

S-2o

The reaction was performed on $0.2 \mathrm{mmol}$ scale with $(R)-\mathbf{A 1}(5 \mathrm{~mol} \%)$ as catalyst at $-78{ }^{\circ} \mathrm{C}$ for $12 \mathrm{~h}$ and then purified by column chromatography (petroleum ether:EtOAc $=20: 1$ ) to afford the products.

(R)-2-methyl-2-phenyl-1,2,3,4-tetrahydroquinoline $((R)-10)$<smiles>C[C@]1(c2ccccc2)CCc2ccccc2N1</smiles>

Yellow oil, $22 \mathrm{mg}, 49 \% .[\alpha]_{\mathrm{D}}{ }^{20}=+134$ (c 1.0, $\left.\mathrm{CHCl}_{3}\right)$. HPLC (Chiralpak IA, hexanes/isopropanol $=97 / 3$, flow rate $=1.0 \mathrm{~mL} / \mathrm{min}, \mathrm{I}=254 \mathrm{~nm}$ ) $\mathrm{t}_{\mathrm{R}}=5.4 \mathrm{~min}$ (major), $5.9 \mathrm{~min}$ (minor), $91 \%$ ee.

(S)-6-bromo-2-methyl-2-phenyl-1,2,3,4-tetrahydroquinoline ((S)-2o)<smiles>C[C@]1(c2ccccc2)CCc2cc(Br)ccc2N1</smiles>

White solid, $28.1 \mathrm{mg}, 46 \% .{ }^{1} \mathrm{H}$ NMR (500 MHz, Chloroform- $d$ ) $\delta 7.37-7.33$ (m, 2H), 7.30 (t, $J=7.7 \mathrm{~Hz}, 2 \mathrm{H}), 7.24-7.19(\mathrm{~m}, 1 \mathrm{H}), 7.11(\mathrm{dd}, J=8.5,2.3 \mathrm{~Hz}, 1 \mathrm{H}), 7.02(\mathrm{~d}, J=2.4 \mathrm{~Hz}, 1 \mathrm{H})$, $6.50(\mathrm{~d}, J=8.5 \mathrm{~Hz}, 1 \mathrm{H}), 4.16(\mathrm{~s}, 1 \mathrm{H}), 2.57(\mathrm{dt}, J=16.4,4.6 \mathrm{~Hz}, 1 \mathrm{H}), 2.29$ (ddd, $J=16.5$, 11.4, $5.2 \mathrm{~Hz}, 1 \mathrm{H}), 2.21(\mathrm{dt}, J=12.9,4.7 \mathrm{~Hz}, 1 \mathrm{H}), 1.88(\mathrm{ddd}, J=12.9,11.4,4.9 \mathrm{~Hz}, 1 \mathrm{H}), 1.58$ (s, 3H). ${ }^{13} \mathrm{C}$ NMR (126 MHz, Chloroform- $d$ ) $\delta$ 147.7, 142.9, 131.7, 129.6, 128.4, 126.5, 125.3, 122.5, 114.9, 108.1, 55.6, 35.0, 30.6, 24.2. $[\alpha]_{\mathrm{D}}{ }^{20}=-55.4\left(\mathrm{c} 1.0, \mathrm{CHCl}_{3}\right)$. HPLC (Chiralpak IC, 
hexanes/isopropanol $=97 / 3$, flow rate $=1.0 \mathrm{~mL} / \mathrm{min}, \mathrm{I}=254 \mathrm{~nm}) \mathrm{t}_{\mathrm{R}}=6.8 \mathrm{~min}($ minor $), 7.5$ min (major), $88 \%$ ee. HRMS (ESI) m/z: $[\mathrm{M}+\mathrm{H}]^{+}$Calcd for $\mathrm{C}_{16} \mathrm{H}_{17} \mathrm{BrN}^{+}$302.0539; Found 302.0540.

\section{Kinetic resolution of DHQ through asymmetric iodonation}<smiles>CC1(c2ccccc2)C=Cc2ccccc2N1</smiles>

$( \pm)-1 \mathrm{a}$

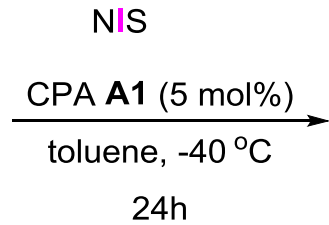

$s=22$<smiles>CC1(c2ccccc2)C=Cc2ccccc2N1</smiles>

$(R)-1 \mathrm{a}$

$42 \%, 84 \%$ ee<smiles>CC1(c2ccccc2)C=Cc2cc(I)ccc2N1</smiles>

(S)-3a

$37 \%, 79 \%$ ee

In a $8 \mathrm{~mL}$ vial containing a magnetic stir bar, was added racemic $1 \mathbf{1 a}(0.1 \mathrm{mmol})$ and CPA (R)-A1 (3.6 mg, $0.01 \mathrm{mmol}, 5 \mathrm{~mol} \%)$ under $\mathrm{N}_{2}$ atmosphere. After adding dry toluene (2.5 mL), the mixture was cooled to $-40{ }^{\circ} \mathrm{C}$ and stirred for 30 minutes. Then a solution of NIS (14 mg, $0.06 \mathrm{mmol}, 0.6$ equiv. $)$ in toluene $(2.5 \mathrm{~mL})$ was added dropwisely at this temperature. After stirring at $-40{ }^{\circ} \mathrm{C}$ for $10 \mathrm{~h}$, the reaction mixture was quenched with saturated $\mathrm{NaHSO}_{3}$ aqueous solution $(1 \mathrm{~mL})$ and then extracted with EtOAc for 3 times. The combined organic layer was washed with brine, dried over $\mathrm{Na}_{2} \mathrm{SO}_{4}$, filtered through Celite and concentrated in vacuo to give a residue, which was purified by column chromatography (petroleum ether:EtOAc $=20: 1$ ) to afford recovered $(R)-\mathbf{1 a}(9 \mathrm{mg}, 42 \%, 84 \%$ ee) and $(S)-\mathbf{3 a}(26 \mathrm{mg}, 37 \%)$.

(S)-6-iodo-2-methyl-2-pheyl-1,2-dihydroquinoline ((S)-3a)<smiles>C[C@]1(c2ccccc2)C=Cc2cc(I)ccc2N1</smiles>

${ }^{1} \mathrm{H}$ NMR (400 MHz, Chloroform- $d$ ) $\delta 7.48(\mathrm{~d}, J=7.7 \mathrm{~Hz}, 2 \mathrm{H}), 7.35(\mathrm{t}, J=7.5 \mathrm{~Hz}, 2 \mathrm{H}), 7.26-$ $7.20(\mathrm{~m}, 2 \mathrm{H}), 7.18(\mathrm{~d}, J=2.0 \mathrm{~Hz}, 1 \mathrm{H}), 6.24(\mathrm{dd}, J=9.0,5.3 \mathrm{~Hz}, 2 \mathrm{H}), 5.60(\mathrm{~d}, J=9.8 \mathrm{~Hz}, 1 \mathrm{H})$, $4.06(\mathrm{~s}, 1 \mathrm{H}), 1.72(\mathrm{~s}, 3 \mathrm{H}) .{ }^{13} \mathrm{C}$ NMR (101 MHz, Chloroform- $d$ ) $\delta$ 148.6, 142.3, 137.3, 135.1, $130.8,128.6,127.2,125.5,122.3,121.7,114.6,57.6,30.3 .[\alpha]_{\mathrm{D}}^{20}=-54.4\left(\mathrm{c} 1.0, \mathrm{CHCl}_{3}\right)$. HPLC $($ Chiralpak IA, hexanes/isopropanol $=97 / 3$, flow rate $=1.0 \mathrm{~mL} / \mathrm{min}, \mathrm{I}=254 \mathrm{~nm}) \mathrm{t}_{\mathrm{R}}=$ 
9.9 min (minor), 10.7 min (major), $79 \%$ ee. HRMS (ESI) m/z: $[\mathrm{M}+\mathrm{H}]^{+}$Calcd for $\mathrm{C}_{16} \mathrm{H}_{15} \mathrm{IN}^{+}$ 348.2044; Found 348.2039.

\section{1 mmol-scale kinetic resolution of DHQ 1a}<smiles>CC1(c2ccccc2)C=Cc2ccccc2N1</smiles>

$( \pm)-1 \mathrm{a}$<smiles>[11CH3]</smiles>

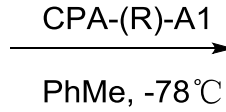

$\mathrm{PhMe},-78^{\circ} \mathrm{C}$<smiles>C[C@]1(c2ccccc2)C=Cc2ccccc2N1</smiles>

$(R)-1 \mathrm{a}$<smiles>C[C@]1(c2ccccc2)C=Cc2cc(Br)ccc2N1</smiles>

$(S)-2 a$

To a $100 \mathrm{~mL}$ vial containing a magnetic stir bar, was added racemic $1 \mathbf{a}(221 \mathrm{mg}, 1.0 \mathrm{mmol})$ and CPA $(R)$-A1 (15.0 mg, $0.02 \mathrm{mmol}, 2 \mathrm{~mol} \%)$ under $\mathrm{N}_{2}$ atmosphere. After adding dry toluene (25 $\mathrm{mL}$ ), the mixture was cooled to $-78{ }^{\circ} \mathrm{C}$ and stirred for 30 minutes. Then a solution of NBS (107 mg, $0.6 \mathrm{mmol}, 0.6$ equiv.) in toluene $(25 \mathrm{~mL})$ was added dropwisely at this temperature. After stirring at $-78{ }^{\circ} \mathrm{C}$ for $20 \mathrm{~h}$, the reaction mixture was quenched with saturated $\mathrm{NaHSO}_{3}$ aqueous solution $(10 \mathrm{~mL})$, extracted with EtOAc for 3 times. The combined organic layer was washed with brine, dried over $\mathrm{Na}_{2} \mathrm{SO}_{4}$, filtered through Celite and concentrated in vacuo to give a residue, which was purified by column chromatography (petroleum ether:EtOAc $=20: 1$ ) to afford recovered $(R)-\mathbf{1 a}(86.6 \mathrm{mg}, 40 \%, 92 \%$ ee) and $(S)-\mathbf{2 a}(120 \mathrm{mg}, 40 \%, 94 \%$ ee).

\section{Transformations of chiral products:}<smiles>C[C@]1(c2ccccc2)C=Cc2cc(Br)ccc2N1</smiles>

$(S)-2 a$ $94 \%$ ee

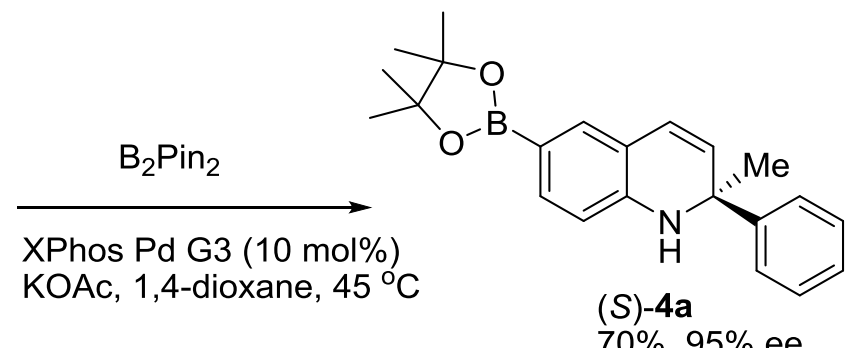

$70 \%, 95 \%$ ee

General procedure ${ }^{3}$ : To a solution of $\mathbf{2 a}(30 \mathrm{mg}, 0.1 \mathrm{mmol}, 94 \%$ ee) in 1,4-dioxane ( $3 \mathrm{~mL})$ was added potassium acetate ( $29 \mathrm{mg}, 0.3 \mathrm{mmol}, 3$ equiv), bis(pinacolato)diboron (127 mg, 0.5 mmol, 5 equiv) and XPhos Pd G3 (8.5 mg, $10 \mathrm{~mol} \%)$ at $\mathrm{rt}$ under $\mathrm{N}_{2}$ atmosphere. After stirring at $45{ }^{\circ} \mathrm{C}$ (heated by an oil bath) for $10 \mathrm{~h}$, EtOAc $(2 \mathrm{~mL})$ and HOAc $(1 \mathrm{~mL})$ were added and 
the mixture was stirred for 30 minutes at rt. Removal of the organic solvents in vacuo provided a residue, which was purified by column chromatography (300-400 mesh silica gel, petroleum ether/EtOAc $=30 / 1)$ to afford the product $4 \mathbf{a}(24 \mathrm{mg}, 70 \%, 95 \%$ ee $)$ as a white solid. ${ }^{1} \mathrm{H}$ NMR (500 MHz, Chloroform- $d$ ) $\delta 7.53-7.44(\mathrm{~m}, 3 \mathrm{H}), 7.36(\mathrm{~d}, J=13.7 \mathrm{~Hz}, 1 \mathrm{H})$, $7.32(\mathrm{~d}, J=7.6 \mathrm{~Hz}, 2 \mathrm{H}), 7.23(\mathrm{t}, J=7.3 \mathrm{~Hz}, 1 \mathrm{H}), 6.41(\mathrm{~d}, J=7.9 \mathrm{~Hz}, 1 \mathrm{H}), 6.34(\mathrm{~d}, J=9.8 \mathrm{~Hz}$, 1H), $5.53(\mathrm{~d}, J=9.7 \mathrm{~Hz}, 1 \mathrm{H}), 4.21(\mathrm{~s}, 1 \mathrm{H}), 1.73(\mathrm{~s}, 3 \mathrm{H}), 1.32(\mathrm{~s}, 12 \mathrm{H}) .{ }^{13} \mathrm{C}$ NMR $(101 \mathrm{MHz}$, Chloroform- $d$ ) $\delta 148.9,145.4,136.3,133.9,129.3,128.6,127.1,125.5,123.3,118.2,111.7$, 83.6, 83.4, 57.8, 30.4, 25.2, 25.0. HPLC (Chiralpak IB, hexanes/isopropanol = 97/3, flow rate $=1.0 \mathrm{~mL} / \mathrm{min}, \mathrm{I}=254 \mathrm{~nm}$ ) $\mathrm{t}_{\mathrm{R}}=5.7 \mathrm{~min}$ (minor), $7.5 \mathrm{~min}$ (major), 95\% ee. HRMS (ESI) m/z: $[\mathrm{M}+\mathrm{H}]^{+}$Calcd for $\mathrm{C}_{22} \mathrm{H}_{27} \mathrm{BNO}_{2}{ }^{+}$348.2129; Found 348.2129.

\section{One-pot stepwise brominative kinetic resolution and boronation}<smiles>[R]CC([R])([Al])Nc1ccccc1C#C</smiles>

$( \pm)-1$

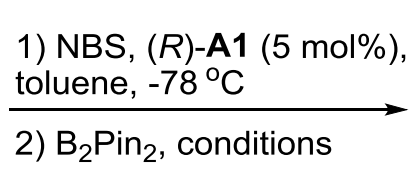

2) $\mathrm{B}_{2} \mathrm{Pin}_{2}$, conditions<smiles>[R]C1Cc2ccccc2NC([R])([18F])C1</smiles>

$(R)-1$<smiles>[R]CC1([Al])Nc2ccc(C[Se][B])cc2CC1[R]</smiles>

(S)-4

The kinetic resolution was performed according to the standard procedure. Due to the challenges in separating the SM and product, the mixture was directly subjected into the boronation reaction.

General procedure: In a $25 \mathrm{~mL}$ vial containing a magnetic stir bar, was added racemic $\mathbf{1}(0.2$ mmol) and CPA (R)-A1 (7.2 mg, $0.01 \mathrm{mmol}, 5 \mathrm{~mol} \%)$ under $\mathrm{N}_{2}$ atmosphere. After adding dry toluene $(5 \mathrm{~mL})$, the mixture was cooled to $-78^{\circ} \mathrm{C}$ and stirred for 30 minutes. Then a solution of NBS (21 mg, $0.12 \mathrm{mmol}, 0.6$ equiv.) in toluene $(5 \mathrm{~mL})$ was added dropwisely at this temperature. After stirring at $-78{ }^{\circ} \mathrm{C}$ for $12 \sim 24 \mathrm{~h}$, the reaction mixture was quenched with saturated $\mathrm{NaHSO}_{3}$ aqueous solution $(1 \mathrm{~mL})$, extracted with EtOAc for 3 times. The combined organic layer was washed with brine, dried over $\mathrm{Na}_{2} \mathrm{SO}_{4}$, filtered through Celite and concentrated in vacuo to give a residue. To a solution of the above residue in 1,4-dioxane (3 $\mathrm{mL}$ ) was added potassium acetate ( $29 \mathrm{mg}, 0.3 \mathrm{mmol}, 3$ equiv), bis(pinacolato)diboron (127 
mg, 0.5 mmol, 5 equiv) and XPhos Pd G3 (8.5 mg, 10 mol\%) at $\mathrm{rt}$ under $\mathrm{N}_{2}$ atmosphere. After stirring at $45{ }^{\circ} \mathrm{C}$ (heated by an oil bath) for $10 \mathrm{~h}$, EtOAc $(2 \mathrm{~mL})$ and HOAc $(1 \mathrm{~mL})$ were added and the mixture was stirred for 30 minutes at rt. Removal of the organic solvents in vacuo provided a residue, which was purified by column chromatography (300-400 mesh silica gel, petroleum ether/EtOAc $=30 / 1$ to $10 / 1)$ to afford the product $(R)-\mathbf{1}$ and $(S)-\mathbf{4}$.

$(R)$-2,3-dimethyl-2-phenyl-1,2-dihydroquinoline $((R)-\mathbf{1 p})$<smiles>CC1=Cc2ccccc2N[C@@]1(C)c1ccccc1</smiles>

Yellow solid, $20.5 \mathrm{mg}, 43 \% .[\alpha]_{\mathrm{D}}^{20}=+10.4\left(\mathrm{c} 1.0, \mathrm{CHCl}_{3}\right)$. HPLC (Chiralpak IB, hexanes/isopropanol $=97 / 3$, flow rate $=1.0 \mathrm{~mL} / \mathrm{min}, \mathrm{I}=254 \mathrm{~nm}) \mathrm{t}_{\mathrm{R}}=5.7 \mathrm{~min}($ minor), 7.5 $\min$ (major), $84 \%$ ee.

(S)-2,3-dimethyl-2-phenyl-6-(4,4,5,5-tetramethyl-1,3,2-dioxaborolan-2-yl)-1,2-dihydroquinol -ine $((S)-\mathbf{4 p})$

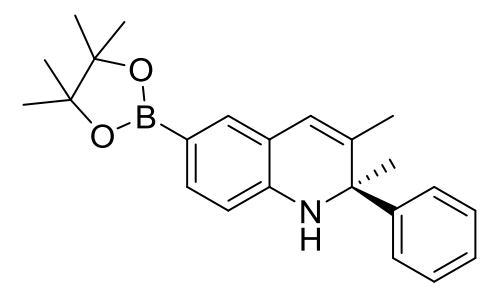

White solid, $28 \mathrm{mg}, 38 \%$. ${ }^{1} \mathrm{H}$ NMR (500 MHz, Chloroform- $d$ ) $\delta 7.56(\mathrm{~d}, J=8.0 \mathrm{~Hz}, 2 \mathrm{H}), 7.41$ $(\mathrm{d}, J=7.9 \mathrm{~Hz}, 1 \mathrm{H}), 7.37-7.30(\mathrm{~m}, 3 \mathrm{H}), 7.26-7.22(\mathrm{~m}, 1 \mathrm{H}), 6.32(\mathrm{~d}, J=7.8 \mathrm{~Hz}, 1 \mathrm{H}), 6.21(\mathrm{~s}$ $1 \mathrm{H}), 4.06(\mathrm{~s}, 1 \mathrm{H}), 1.77$ (d, $J=1.3 \mathrm{~Hz}, 3 \mathrm{H}), 1.56(\mathrm{~s}, 3 \mathrm{H}), 1.33$ (d, $J=1.5 \mathrm{~Hz}, 12 \mathrm{H}) .{ }^{13} \mathrm{C}$ NMR (126 MHz, Chloroform- $d$ ) $\delta 147.2,144.7,135.6,135.5,132.8,128.4,127.2,126.5,121.8$, $119.2,111.2,83.3,61.0,26.6,25.0,25.0,19.6 . \quad[\alpha]_{D}^{20}=-5.4(\mathrm{c} 1.0$, Chloroform- $d)$. HPLC $($ Chiralpak IB, hexanes/isopropanol $=97 / 3$, flow rate $=1.0 \mathrm{~mL} / \mathrm{min}, \mathrm{I}=254 \mathrm{~nm}) \mathrm{t}_{\mathrm{R}}=6.2 \mathrm{~min}$ (major), 8.5 min (minor), $85 \%$ ee. HRMS (ESI) m/z: $[\mathrm{M}+\mathrm{H}]^{+}$Calcd for $\mathrm{C}_{23} \mathrm{H}_{29} \mathrm{BNO}_{2}{ }^{+} 362.2286$; Found 362.2286 .

(R)-2-(3,5-dimethylphenyl)-2-methyl-1,2-dihydroquinoline ((R)-1q) 


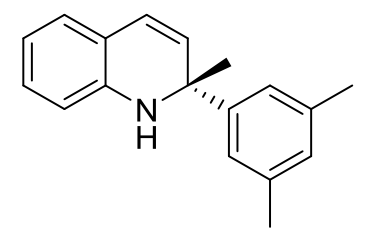

White solid, $21.2 \mathrm{mg}, 42 \% .[\alpha]_{\mathrm{D}}^{20}=+18.2\left(\mathrm{c} 1.0, \mathrm{CHCl}_{3}\right)$. HPLC (Chiralpak IC, hexanes/isopropanol $=97 / 3$, flow rate $=1.0 \mathrm{~mL} / \mathrm{min}, \mathrm{I}=254 \mathrm{~nm}$ ) $\mathrm{t}_{\mathrm{R}}=5.0 \mathrm{~min}$ (major), $5.3 \mathrm{~min}$ (minor), $91 \%$ ee.

(S)-2-(3,5-dimethylphenyl)-2-methyl-6-(4,4,5,5-tetramethyl-1,3,2-dioxaborolan-2-yl)-1,2-dih ydroquinoline $((S)-\mathbf{4 q})$

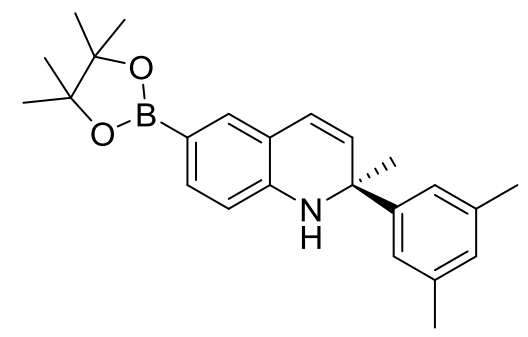

White solid, $29.4 \mathrm{mg}, 39 \% .{ }^{1} \mathrm{H}$ NMR $\left(500 \mathrm{MHz}, \mathrm{CDCl}_{3}\right) \delta 7.46(\mathrm{~d}, J=7.9 \mathrm{~Hz}, 1 \mathrm{H}), 7.37(\mathrm{~s}$, 1H), $7.10(\mathrm{~s}, 2 \mathrm{H}), 6.88(\mathrm{~s}, 1 \mathrm{H}), 6.41(\mathrm{~d}, J=7.9 \mathrm{~Hz}, 1 \mathrm{H}), 6.33(\mathrm{~d}, J=9.8 \mathrm{~Hz}, 1 \mathrm{H}), 5.50(\mathrm{~d}, J=$ $9.7 \mathrm{~Hz}, 1 \mathrm{H}), 4.15(\mathrm{~s}, 1 \mathrm{H}), 2.30(\mathrm{~s}, 6 \mathrm{H}), 1.70(\mathrm{~s}, 3 \mathrm{H}), 1.32$ (s, 12H). ${ }^{13} \mathrm{C}$ NMR $(126 \mathrm{MHz}$, $\left.\mathrm{CDCl}_{3}\right) \delta 148.9,145.4,138.1,136.2,133.8,129.4,128.7,123.5,123.0,118.1,111.7,83.3$, 57.7, 30.5, 25.0, 21.6. $[\alpha]_{\mathrm{D}}{ }^{20}=-24.8\left(\mathrm{c} 1.0, \mathrm{CHCl}_{3}\right)$. HPLC (Chiralpak IC, hexanes/isopropanol $=97 / 3$, flow rate $=1.0 \mathrm{~mL} / \mathrm{min}, \mathrm{I}=210 \mathrm{~nm}$ ) $t_{R}=7.1 \mathrm{~min}$ (major), $8.3 \mathrm{~min}$ (minor), $85 \%$ ee. HRMS (ESI) m/z: $[\mathrm{M}+\mathrm{H}]^{+}$Calcd for $\mathrm{C}_{24} \mathrm{H}_{31} \mathrm{BNO}_{2}{ }^{+}$376.2442; Found 376.2441.

(2R,3R,4R)-6-bromo-3-iodo-4-methoxy-2-methyl-2-phenyl-1,2,3,4-tetrahydroquinoline $((S)-5 a)$<smiles>[M]C1(c2ccccc2)C=Cc2cc(Br)ccc2N1</smiles>

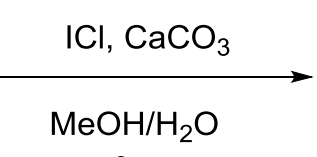
$-30{ }^{\circ} \mathrm{C} \sim 0{ }^{\circ} \mathrm{C}$

(S)-2a, 96\% ee<smiles>COC1c2cc(Br)ccc2NC(C)(c2ccccc2)C1I</smiles>

(S)-5a $95 \%, 10: 1 \mathrm{dr}, 95 \%$ ee 
To a solution of $(S)-\mathbf{2 a}(24.9 \mathrm{mg}, 0.083 \mathrm{mmol}, 1.0$ equiv.) in $\mathrm{MeOH}(1.5 \mathrm{~mL})$ was successively added $\mathrm{H}_{2} \mathrm{O}(0.3 \mathrm{~mL}), \mathrm{CaCO}_{3}(12.4 \mathrm{mg}, 0.124 \mathrm{mmol}, 1.5$ equiv. $)$ at rt. After cooling the mixture to $-30{ }^{\circ} \mathrm{C}$, a solution of $\mathrm{ICl}(14.1 \mathrm{mg}, 0.087 \mathrm{mmol}, 1.05$ equiv.) in $\mathrm{MeOH}$ $(1.0 \mathrm{~mL})$ was added dropwisely at this temperature then the mixture was slowly warmed to 0 ${ }^{\circ} \mathrm{C}$. After stirring for $30 \mathrm{~min}$ at $0{ }^{\circ} \mathrm{C}$, the mixture was quenched with saturated sodium thiosulfate aqueous solution, extracted with EtOAc for 3 times. The combined organic layer was washed with brine, dried over $\mathrm{Na}_{2} \mathrm{SO}_{4}$, filtered and concentrated in vacuo to give a residue, which was purified by flash chromatography $(300 \sim 400$ mesh silica gel, $\mathrm{PE} / \mathrm{EtOAc}=$ 20/1) to afford the product $(S)-\mathbf{5 a}$ as a light yellow solid $(36.1 \mathrm{mg}, 95 \%, \mathrm{dr}=10 / 1,95 \%$ ee)

${ }^{1} \mathrm{H}$ NMR (500 MHz, Chloroform- $\left.d\right) \delta 7.42-7.33(\mathrm{~m}, 2 \mathrm{H}), 7.34-7.25(\mathrm{~m}, 3 \mathrm{H}), 7.27-7.20$ $(\mathrm{m}, 2 \mathrm{H}), 6.55(\mathrm{dd}, J=8.6,2.0 \mathrm{~Hz}, 1 \mathrm{H}), 4.98(\mathrm{~d}, J=5.3 \mathrm{~Hz}, 1 \mathrm{H}), 4.74(\mathrm{~d}, J=5.4 \mathrm{~Hz}, 1 \mathrm{H})$, $4.25(\mathrm{~s}, 1 \mathrm{H}), 3.15$ (s, 3H), 1.77 (s, 3H). ${ }^{13} \mathrm{C}$ NMR (126 MHz, Chloroform- $d$ ) $\delta$ 144.1, 141.9, $132.9,132.4,128.4,127.4,125.8,120.0,115.9,109.6,81.9,59.0,55.7,40.3,30.6 .[\alpha]_{\mathrm{D}}{ }^{20}=$ -124 (c 1.0, $\mathrm{CHCl}_{3}$ ). HPLC (Chiralpak IA, hexanes/isopropanol $=90 / 10$, flow rate $=1.0$ $\mathrm{mL} / \mathrm{min}, \mathrm{I}=254 \mathrm{~nm}) \mathrm{t}_{\mathrm{R}}=6.7 \min ($ minor), $8.5 \min ($ major), $95 \%$ ee. HRMS $(\mathrm{ESI}) \mathrm{m} / \mathrm{z}$ : $[\mathrm{M}+\mathrm{H}]^{+}$Calcd for $\mathrm{C}_{17} \mathrm{H}_{18} \mathrm{BrINO}^{+}$457.9611; Found 457.9603.

(S)-6-bromo-8-iodo-2-methyl-2-phenyl-1,2,3,4-tetrahydroquinoline $((S)$-6o)

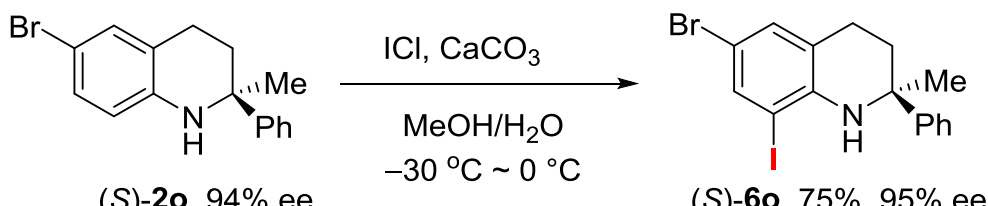

(S)-20, $94 \%$ ee

(S)-6o, $75 \%, 95 \%$ ee

To a solution of $(S)-\mathbf{2 0}(28.0 \mathrm{mg}, 0.093 \mathrm{mmol}, 1.0$ equiv., $94 \%$ ee $)$ in $\mathrm{MeOH}(1.5 \mathrm{~mL})$ was successively added $\mathrm{H}_{2} \mathrm{O}(0.3 \mathrm{~mL})$ and $\mathrm{CaCO}_{3}(14.0 \mathrm{mg}, 0.14 \mathrm{mmol}, 1.5$ equiv. $)$ at rt. After cooling the mixture to $-30{ }^{\circ} \mathrm{C}$, a solution of $\mathrm{ICl}(14.2 \mathrm{mg}, 0.088 \mathrm{mmol}, 0.95$ equiv.) in $\mathrm{MeOH}$ $(1.0 \mathrm{~mL})$ was added dropwisely then the mixture was slowly warmed to $0{ }^{\circ} \mathrm{C}$. After stirring at $0{ }^{\circ} \mathrm{C}$ for $5 \mathrm{~h}$, the reaction mixture was quenched with saturated sodium thiosulfate aqueous solution, extracted with EtOAc for 3 times. The combined organic layer was washed with brine, dried over $\mathrm{Na}_{2} \mathrm{SO}_{4}$, filtered and concentrated in vacuo to give a residue, which was 
purified by flash chromatography $(300 \sim 400$ mesh silica gel, PE/EtOAc $=100 / 1)$ to afford the product $(S)-60(29.8 \mathrm{mg}, 75 \%, 95 \%$ ee $)$ as colorless oil. ${ }^{1} \mathrm{H}$ NMR (500 MHz, Chloroform- $\left.d\right) \delta$ $7.63(\mathrm{~s}, 1 \mathrm{H}), 7.31(\mathrm{t}, J=7.5 \mathrm{~Hz}, 2 \mathrm{H}), 7.28-7.19(\mathrm{~m}, 3 \mathrm{H}), 6.99(\mathrm{~s}, 1 \mathrm{H}), 4.66(\mathrm{~s}, 1 \mathrm{H}), 2.53(\mathrm{dt}, J$ $=16.3,4.5 \mathrm{~Hz}, 1 \mathrm{H}), 2.34-2.18(\mathrm{~m}, 2 \mathrm{H}), 1.86(\mathrm{td}, J=12.3,4.7 \mathrm{~Hz}, 1 \mathrm{H}), 1.63(\mathrm{~s}, 3 \mathrm{H}) .{ }^{13} \mathrm{C} \mathrm{NMR}$ (126 MHz, Chloroform- $d$ ) $\delta 147.2,142.4,138.1,131.9,128.7,126.8,125.3,123.2,108.1,84.0$, 57.0, 34.9, 31.2, 25.0. $[\alpha]_{\mathrm{D}}{ }^{20}=-231\left(\mathrm{c} 1.0, \mathrm{CHCl}_{3}\right)$. HPLC (Chiralpak IB, hexanes/isopropanol $=95 / 5$, flow rate $=1.0 \mathrm{~mL} / \mathrm{min}, \mathrm{I}=254 \mathrm{~nm}$ ) $\mathrm{t}_{\mathrm{R}}=4.6 \mathrm{~min}$ (major), $5.5 \mathrm{~min}$ (minor), $95 \%$ ee. HRMS (ESI) m/z: [M+H] $]^{+}$Calcd for $\mathrm{C}_{16} \mathrm{H}_{16} \mathrm{BrIN}^{+}$427.9505; Found 427.9499.

\section{References:}

1. Chen, Y.; Zhu, C.; Guo, Z.; Liu, W.; Yang, X., Asymmetric Synthesis of Hydroquinolines with $\alpha, \alpha$-Disubstitution through Organocatalyzed Kinetic Resolution. Angew. Chem. Int. Ed. 2021, 60, 5268-5272.

2. Negishi, E.; Swanson, D. R.; Rousset, C. J., Clean and convenient procedure for converting primary alkyl iodides and .alpha.,.omega.-diiodoalkanes into the corresponding alkyllithium derivatives by treatment with tert-butyllithium. J. Org. Chem. 1990, 55, 5406-5409.

3. Heparegenix, G.; Praefke, B. K., P.; Selig, R.; Albrecht, W.; Laufer, S., WO2019/149738, 2019, A1. 


\section{X-Ray structures}

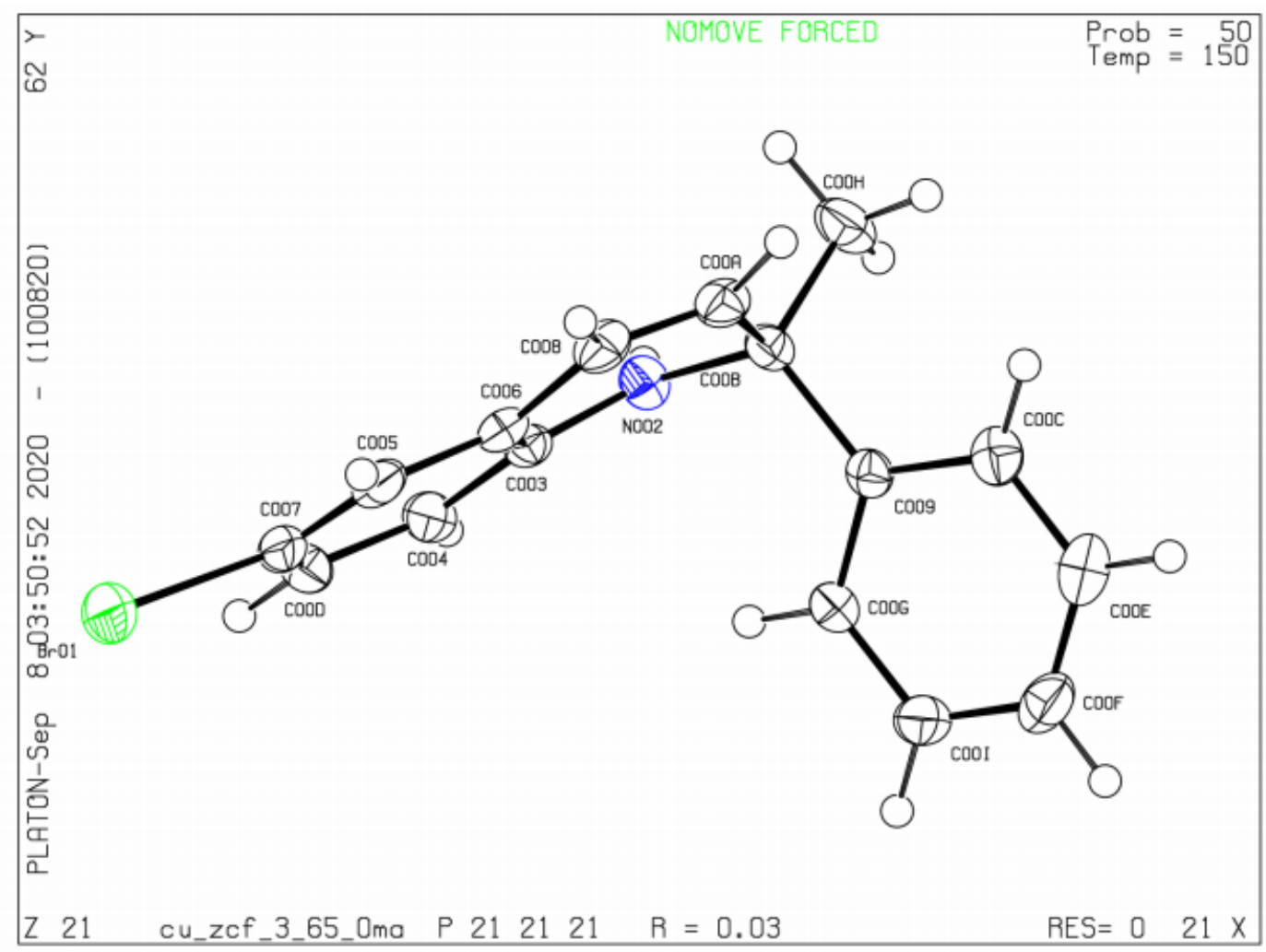

X-ray structure of (S)-2a (CCDC number 2067699, ellipsoids: 50\% probability)

The single crystal of (S)-2a was cultivated through the slow volatilization method using dichloromethane as solvents. 
Table S1. Crystal data for $(S)-\mathbf{2 a}$

\begin{tabular}{|c|c|}
\hline Identification code & cu_zcf_3_65_0ma \\
\hline Empirical formula & $\mathrm{C}_{16} \mathrm{H}_{14} \mathrm{BrN}$ \\
\hline Formula weight & 300.19 \\
\hline Temperature/K & 150.0 \\
\hline Crystal system & orthorhombic \\
\hline Space group & $\mathrm{P} 22_{1} 2_{1}$ \\
\hline $\mathrm{a} / \AA$ & $5.6978(3)$ \\
\hline $\mathrm{b} / \AA$ & $11.8696(6)$ \\
\hline $\mathrm{c} / \AA ̊ \AA$ & $19.8309(11)$ \\
\hline$\alpha /{ }^{\circ}$ & 90 \\
\hline$\beta /{ }^{\circ}$ & 90 \\
\hline$\gamma /{ }^{\circ}$ & 90 \\
\hline Volume $/ \AA^{3}$ & $1341.18(12)$ \\
\hline $\mathrm{Z}$ & 4 \\
\hline$\rho_{\text {calc }} \mathrm{g} / \mathrm{cm}^{3}$ & 1.487 \\
\hline$\mu / \mathrm{mm}^{-1}$ & 4.003 \\
\hline $\mathrm{F}(000)$ & 608.0 \\
\hline Crystal size $/ \mathrm{mm}^{3}$ & $0.2 \times 0.15 \times 0.1$ \\
\hline Radiation & $\mathrm{CuK} \alpha(\lambda=1.54178)$ \\
\hline $2 \Theta$ range for data collection ${ }^{\circ}$ & 8.682 to 158.26 \\
\hline Index ranges & $-6 \leq \mathrm{h} \leq 7,-15 \leq \mathrm{k} \leq 15,-25 \leq 1 \leq 25$ \\
\hline Reflections collected & 39054 \\
\hline Independent reflections & $2871\left[\mathrm{R}_{\mathrm{int}}=0.0360, \mathrm{R}_{\mathrm{sigma}}=0.0163\right]$ \\
\hline
\end{tabular}




\section{HPLC traces}

(R)-2-methyl-2-phenyl-1,2-dihydroquinoline ((R)-1a)<smiles>C[C@]1(c2ccccc2)C=Cc2ccccc2N1</smiles>
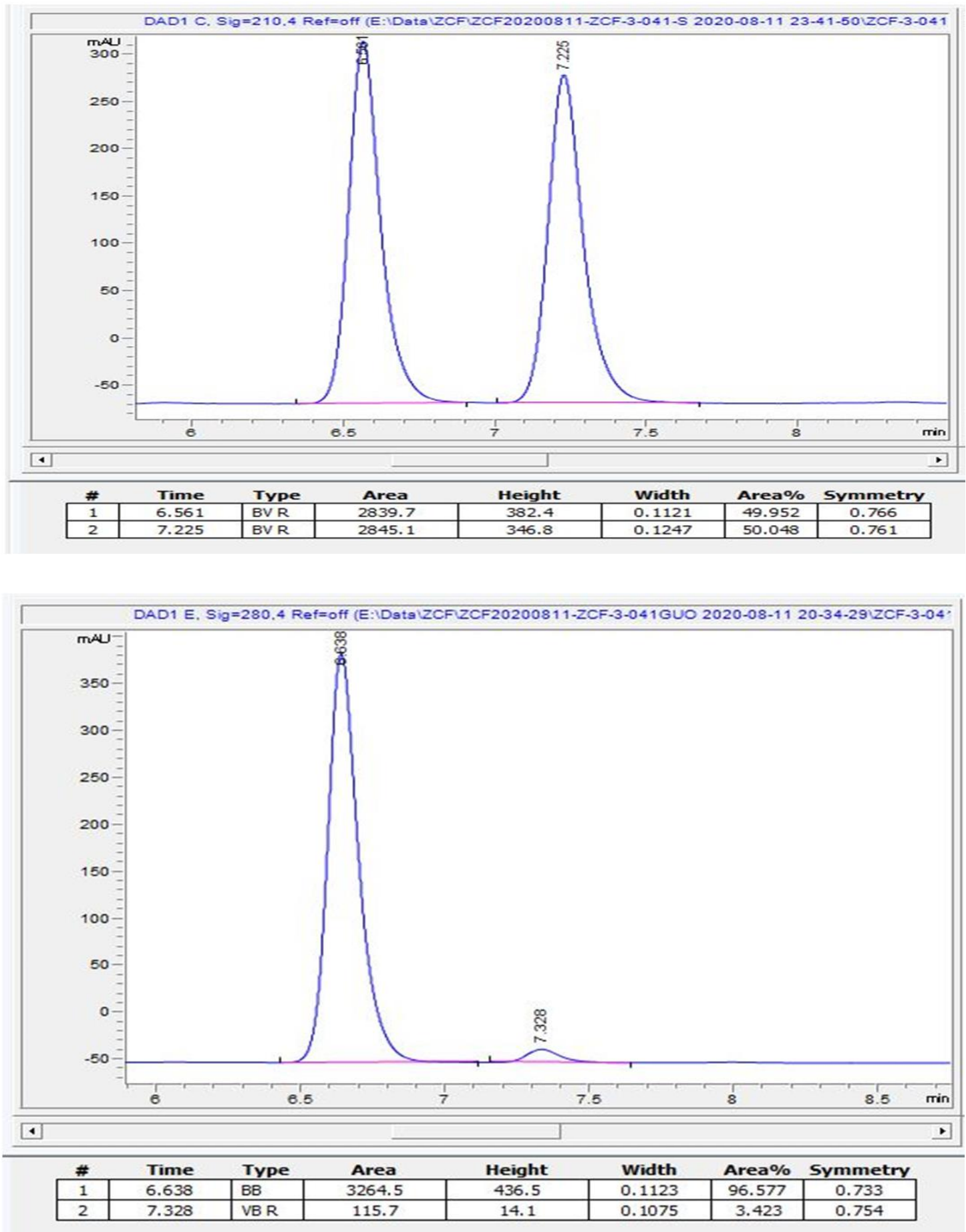
(S)-6-bromo-2-methyl-2-phenyl-1,2-dihydroquinoline ((S)-2a)<smiles>C[C@]1(c2ccccc2)C=Cc2cc(Br)ccc2N1</smiles>

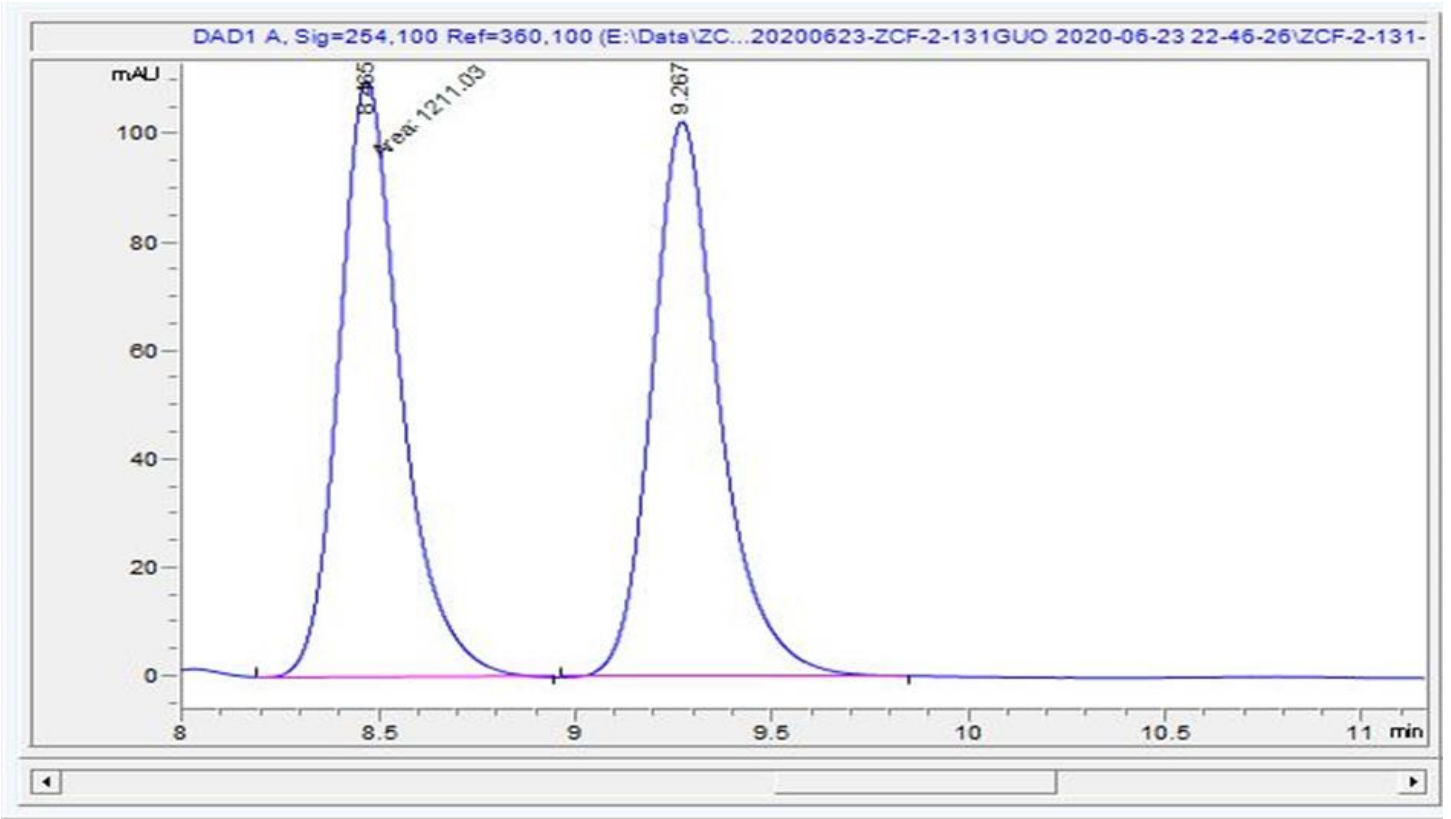

\begin{tabular}{|c|c|c|c|c|c|c|c|}
\hline \# & Time & Type & Area & Height & Width & Area\% & Symmetry \\
\hline 1 & 8.465 & FM & 1211 & 110.3 & 0.183 & 49.699 & 0.774 \\
\hline 2 & 9.267 & $B B$ & 1225.7 & 102.6 & 0.1793 & 50.301 & 0.76 \\
\hline
\end{tabular}

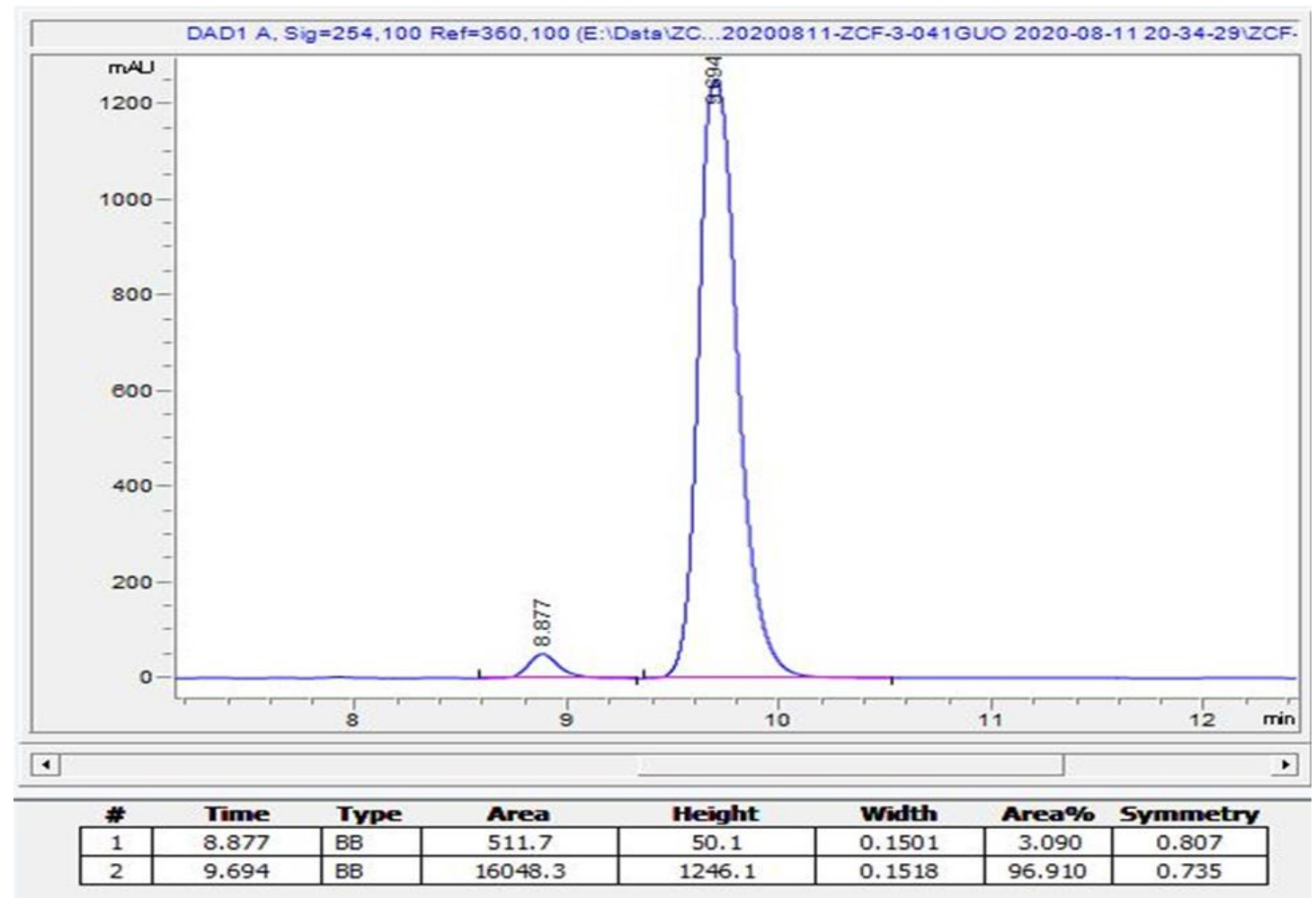


(R)-2-methyl-2-(p-tolyl)-1,2-dihydroquinoline ((R)-1b)
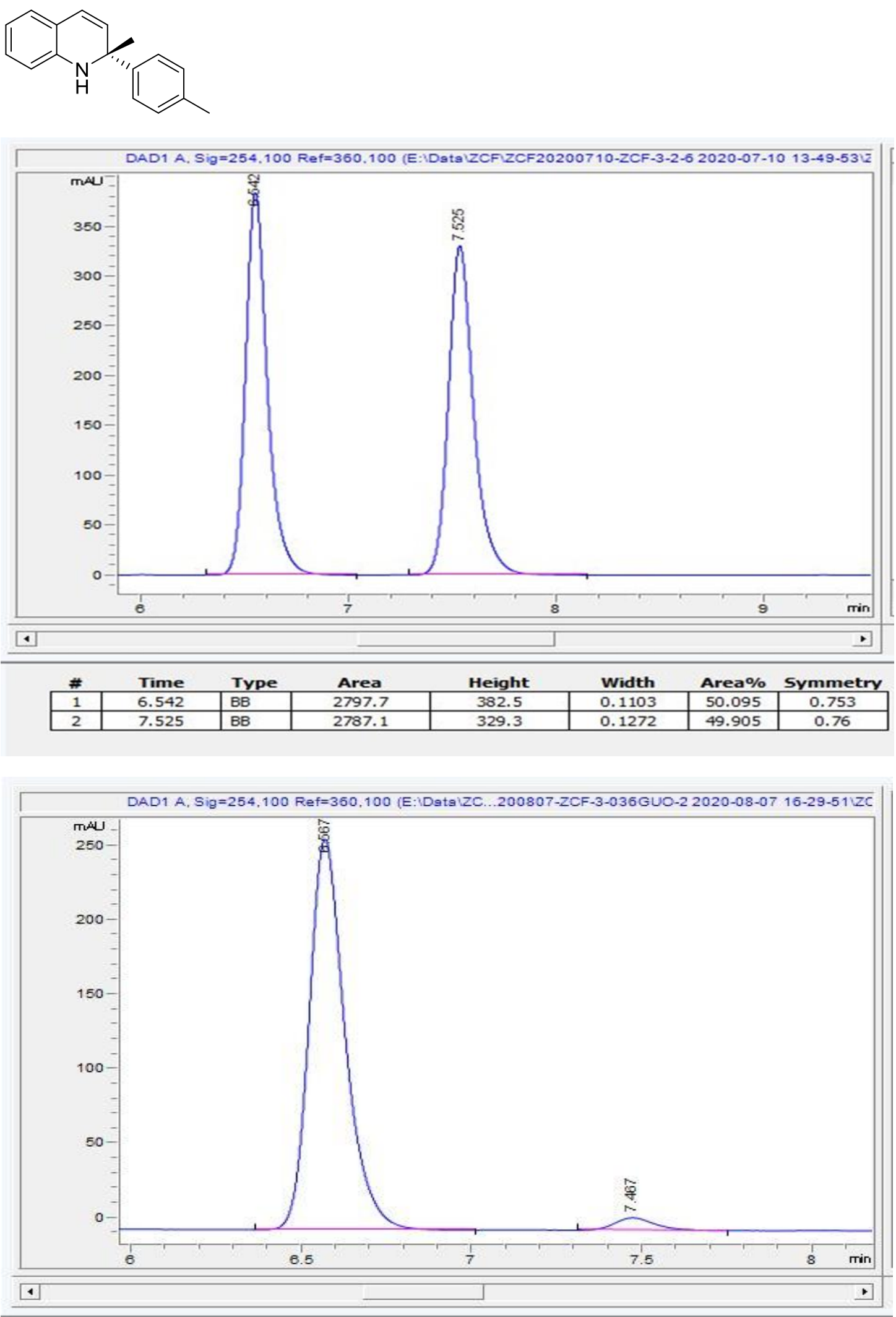

\begin{tabular}{|c|c|l|c|c|c|c|c|} 
\# & Time & \multicolumn{1}{c}{ Type } & Area & Height & \multicolumn{1}{c}{ Width } & \multicolumn{2}{c}{ Area\% } \\
\hline 1 & 6.567 & BB & 1934.1 & 263.2 & 0.1102 & 96.532 & 0.732 \\
\hline 2 & 7.467 & BB & 69.5 & 8.4 & 0.098 & 3.468 & 0.755 \\
\hline
\end{tabular}


(S)-6-bromo-2-methyl-2-(p-tolyl)-1,2-dihydroquinoline ((S)-2b)<smiles>Cc1ccc([C@]2(C)C=Cc3cc(Br)ccc3N2)cc1</smiles>

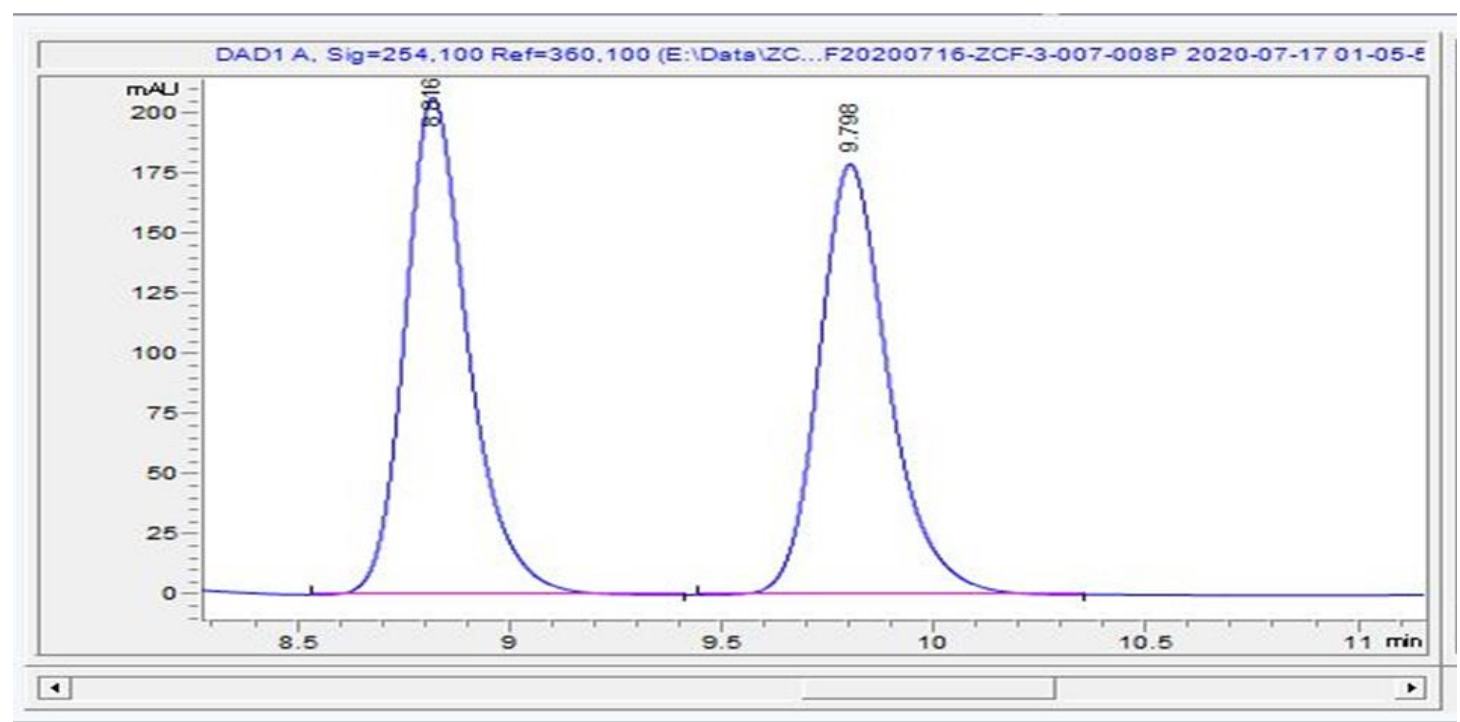

\begin{tabular}{|c|c|l|c|c|c|c|c|}
\hline & Time & \multicolumn{1}{c}{ Type } & Area & Height & \multicolumn{1}{c}{ Width } & \multicolumn{1}{c}{ Area\% Symmetry } \\
\hline 1 & 8.816 & BB & 2153.7 & 208.2 & 0.1567 & 51.173 & 0.75 \\
\hline 2 & 9.798 & BB & 2055 & 180.3 & 0.1715 & 48.827 & 0.78 \\
\hline
\end{tabular}

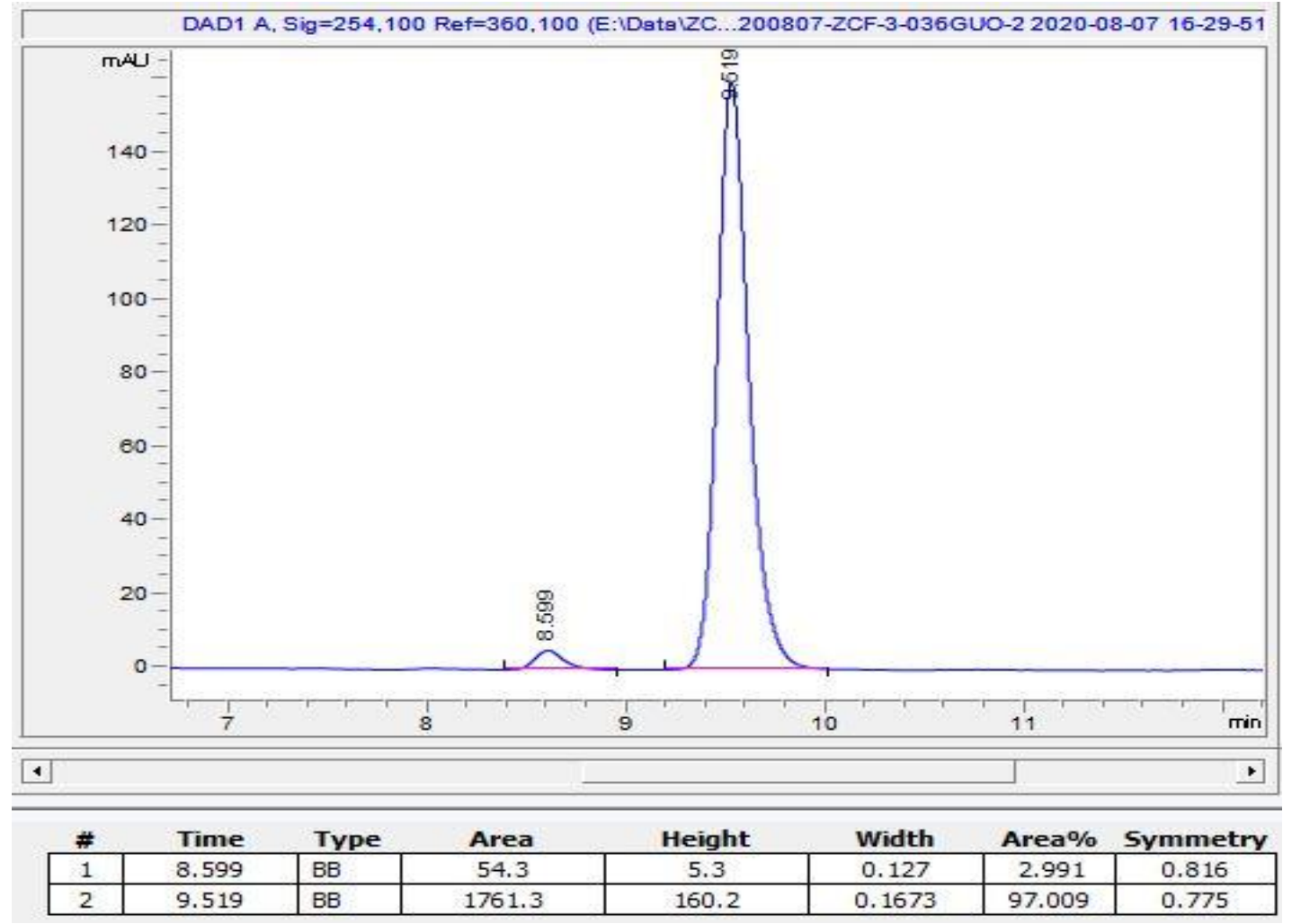


(R)-2-(4-(tert-butyl)phenyl)-2-methyl-1,2-dihydroquinoline $((R)-1 \mathbf{c})$<smiles>CC(C)(C)c1ccc([C@]2(C)C=Cc3ccccc3N2)cc1</smiles>
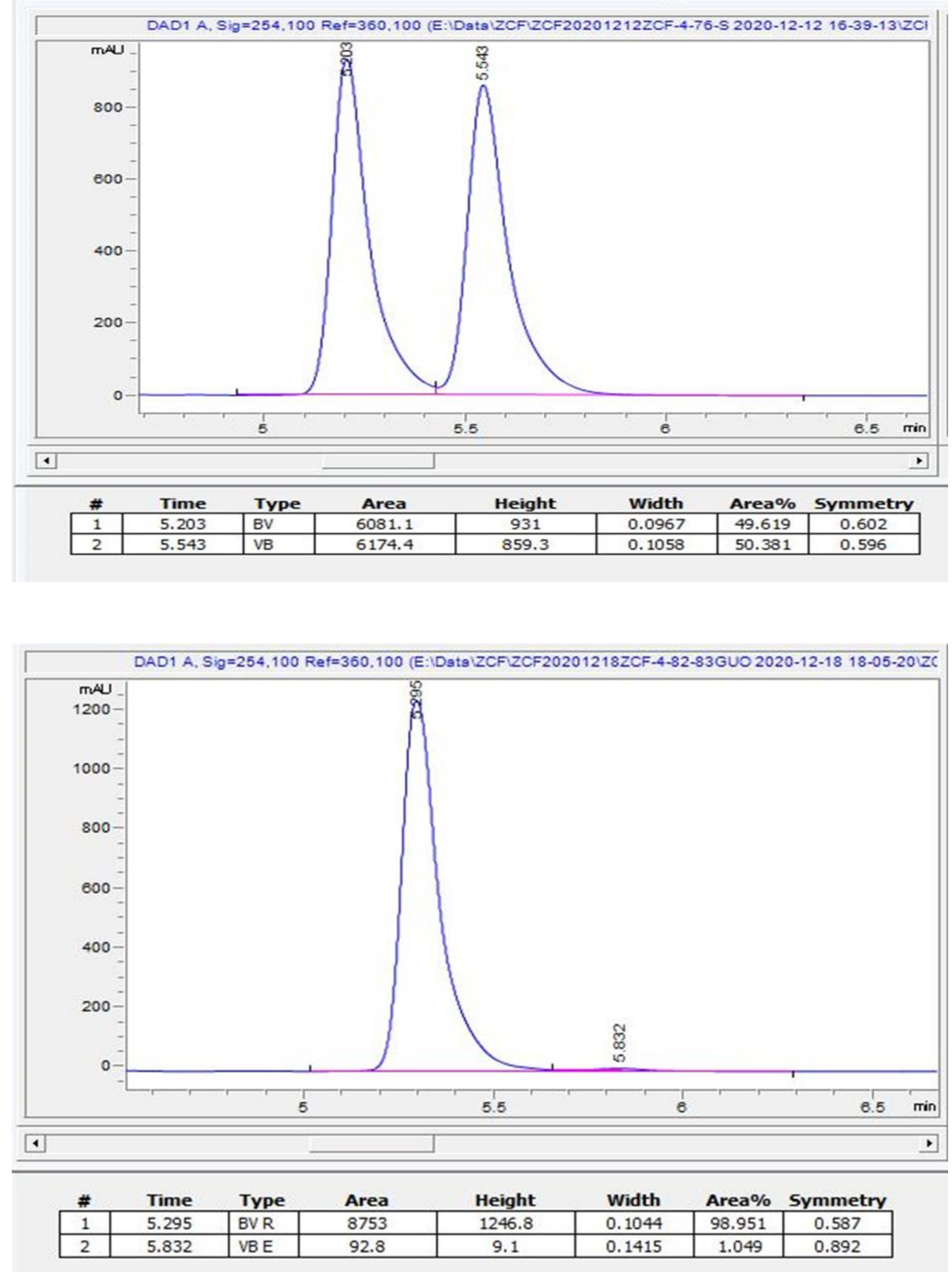
(S)-6-bromo-2-(4-(tert-butyl)phenyl)-2-methyl-1,2-dihydroquinoline ((S)-2c)<smiles>CC(C)(C)c1ccc([C@]2(C)C=Cc3cc(Br)ccc3N2)cc1</smiles>

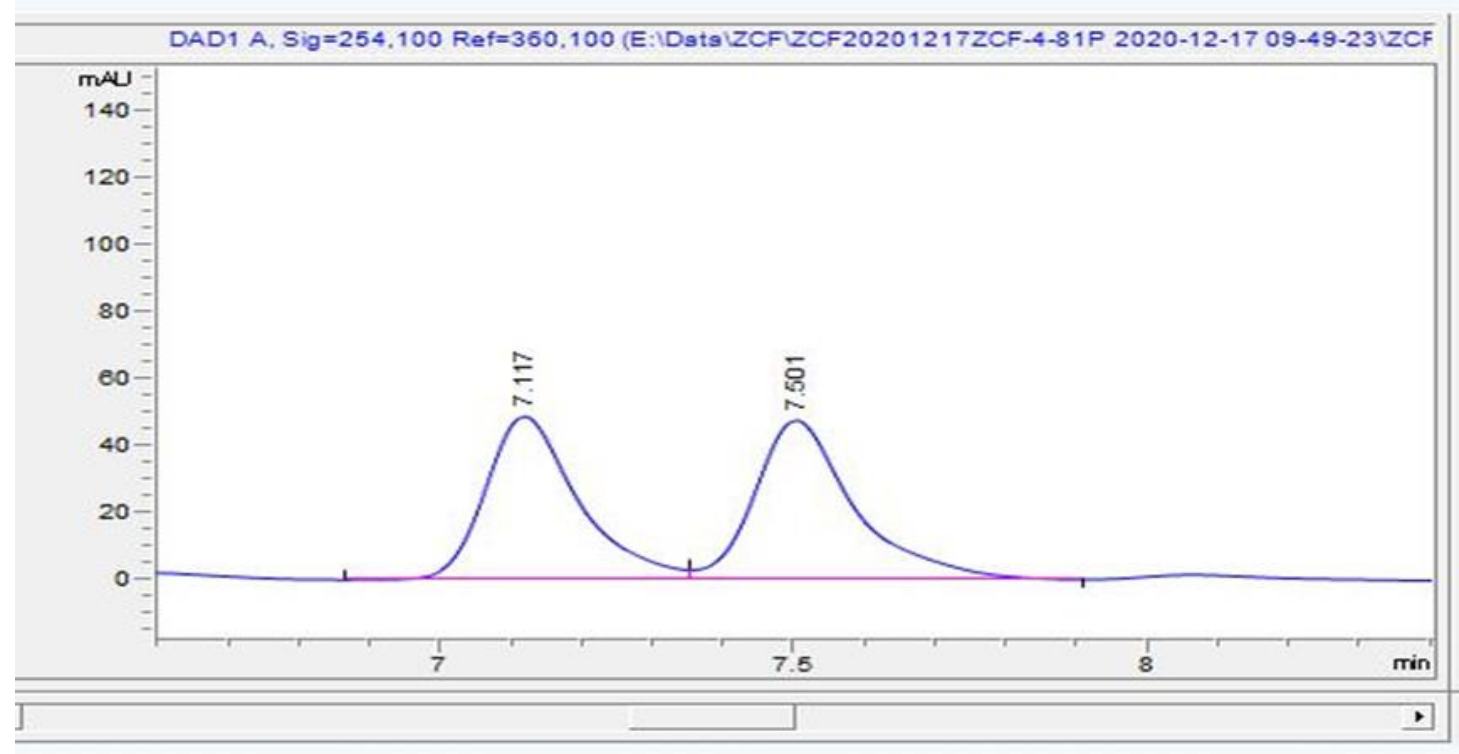

\begin{tabular}{|c|c|l|c|c|c|c|c|}
\multicolumn{1}{r}{ \# } & Time & \multicolumn{1}{c}{ Type } & Area & \multicolumn{1}{c}{ Height } & \multicolumn{2}{c}{ Width } & \multicolumn{2}{c}{ Area\% $\%$ Symmetry } \\
\hline 1 & 7.117 & BV & 446.7 & 48.8 & 0.1364 & 48.905 & 0.679 \\
\hline 2 & 7.501 & VB & 466.7 & 47.6 & 0.1449 & 51.095 & 0.669 \\
\hline
\end{tabular}

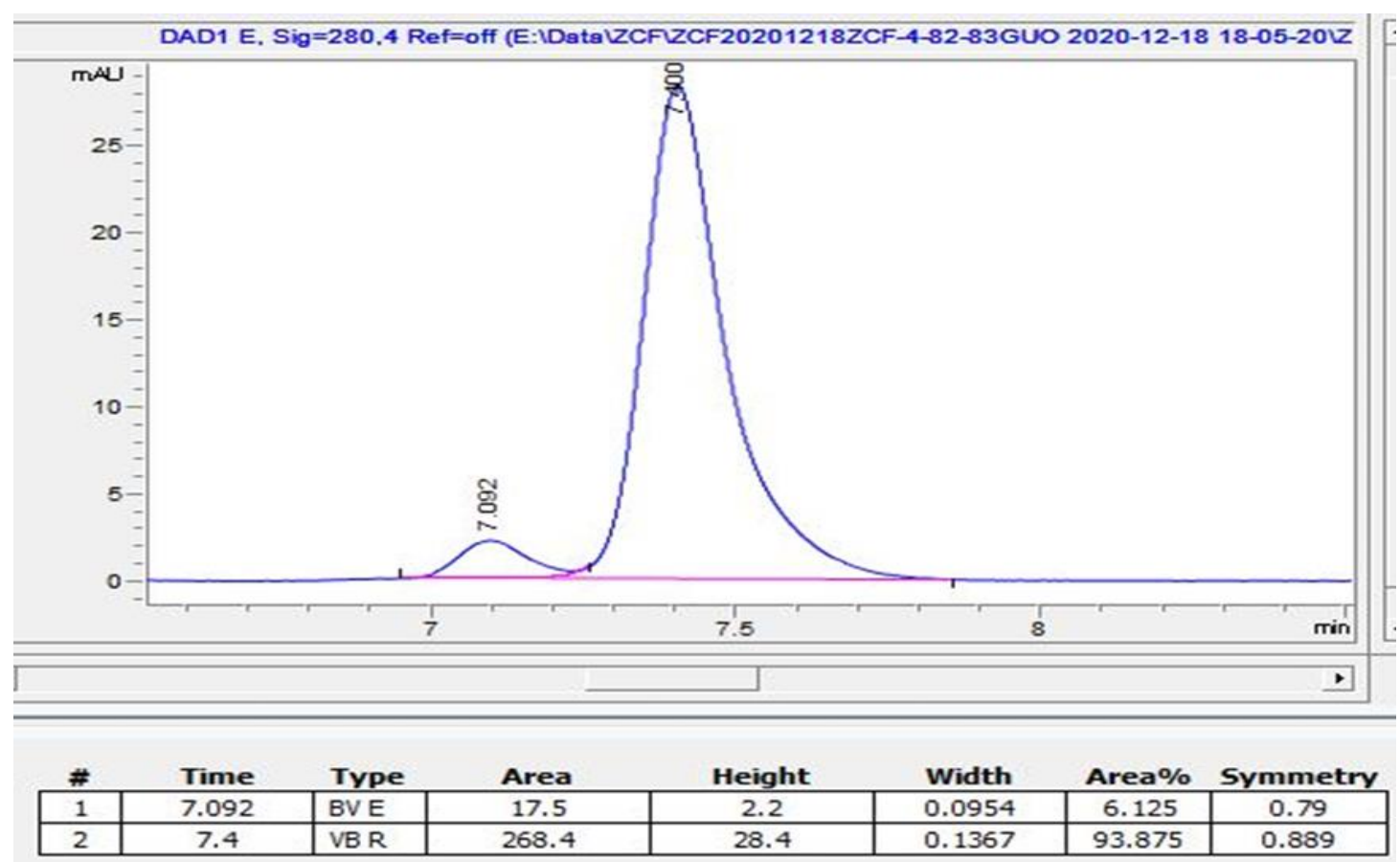


$(R)$-2-([1,1'-biphenyl]-4-yl)-2-methyl-1,2-dihydroquinoline $((R)-\mathbf{1 d})$<smiles>C[C@]1(c2ccc(-c3ccccc3)cc2)C=Cc2ccccc2N1</smiles>
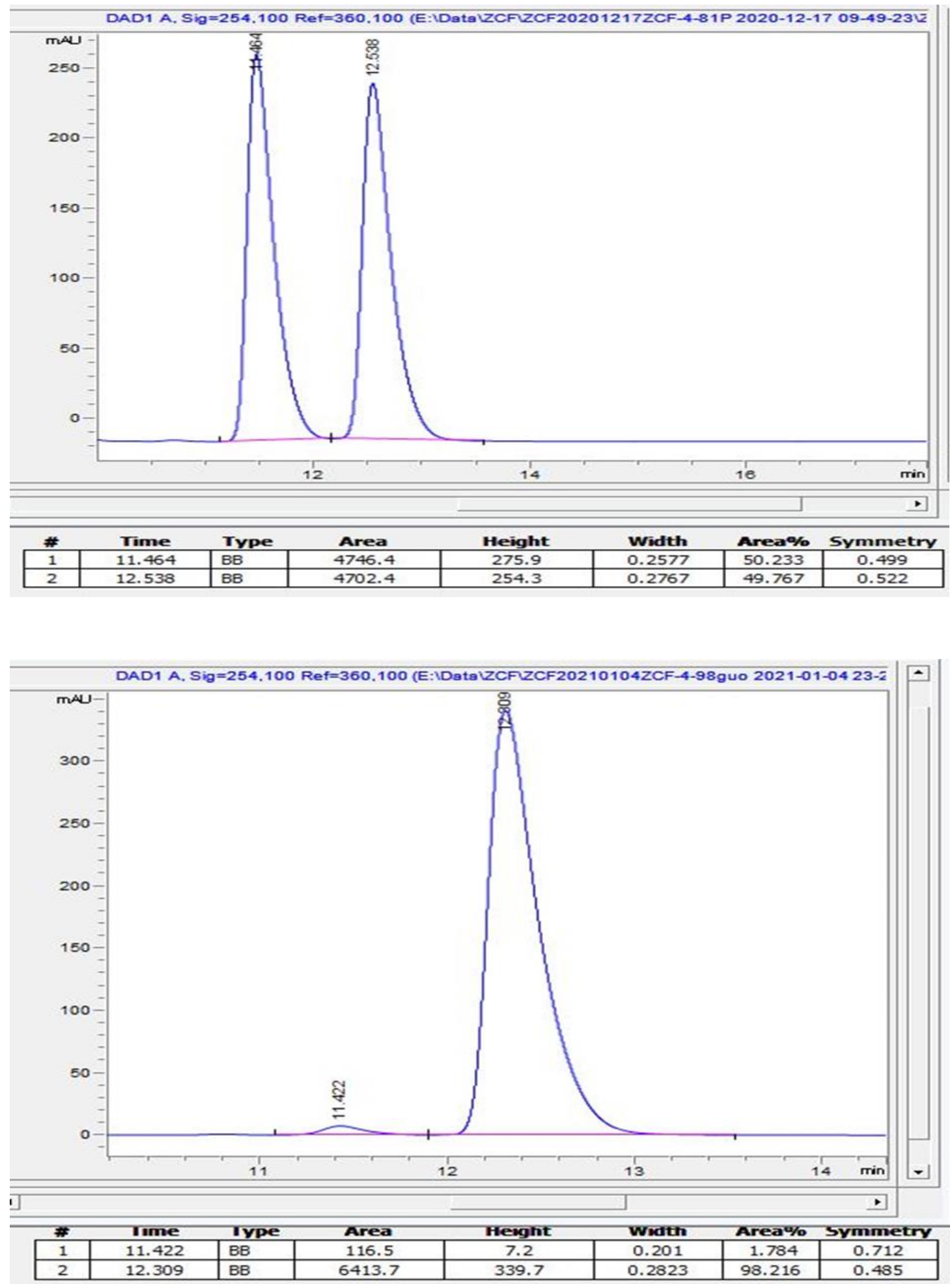
(S)-2-([1,1'-biphenyl]-4-yl)-6-bromo-2-methyl-1,2-dihydroquinoline ((S)-2d)<smiles>C[C@]1(c2ccc(-c3ccccc3)cc2)C=Cc2cc(Br)ccc2N1</smiles>
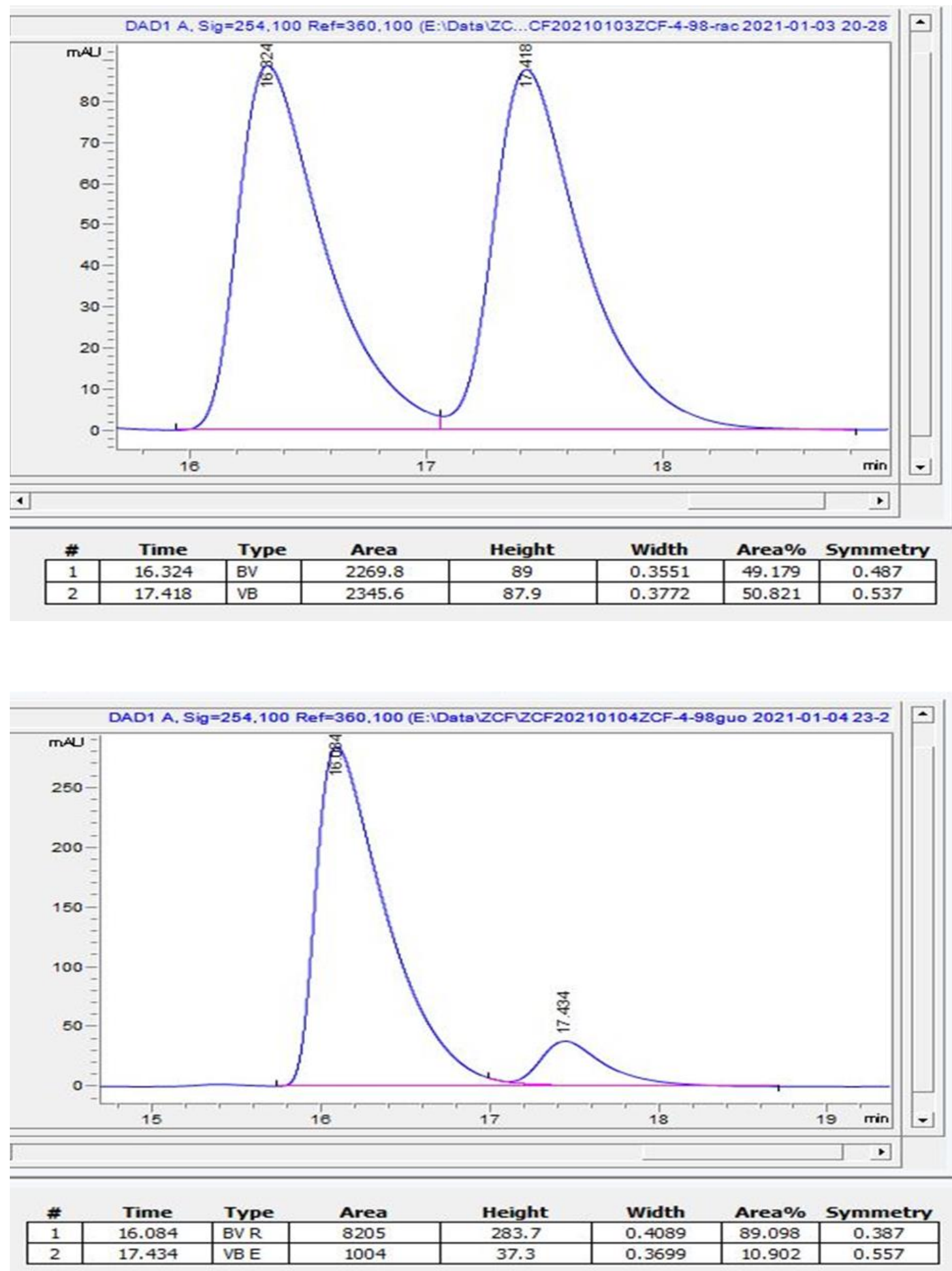
(R)-2-(4-methoxyphenyl)-2-methyl-1,2-dihydroquinoline $((R)-\mathbf{1 e})$<smiles>COc1ccc(C2(C)C=Cc3ccccc3N2)cc1</smiles>
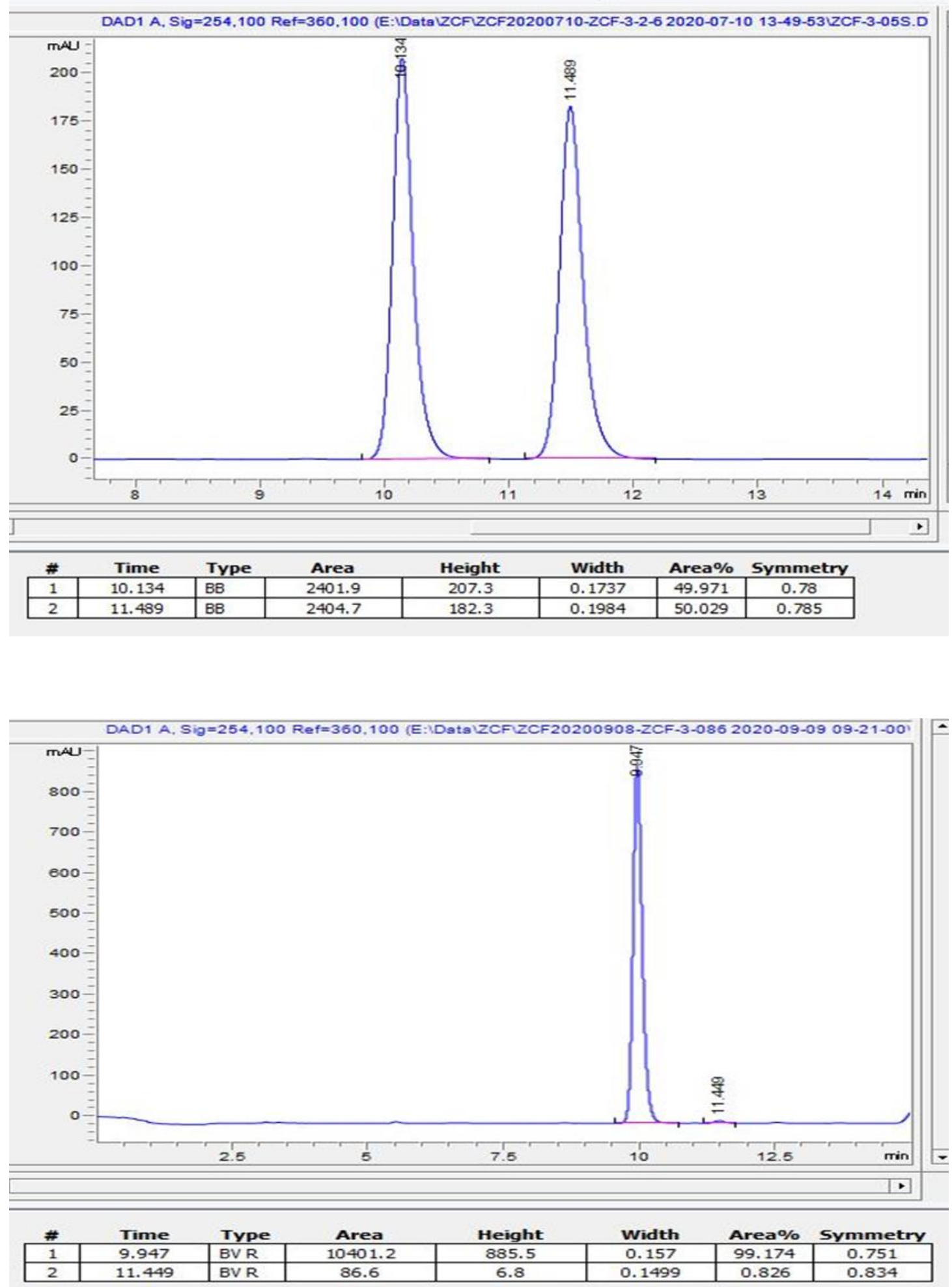
(S)-6-bromo-2-(4-methoxyphenyl)-2-methyl-1,2-dihydroquinoline ((S)-2e)<smiles>COc1ccc([C@]2(C)C=Cc3cc(Br)ccc3N2)cc1</smiles>
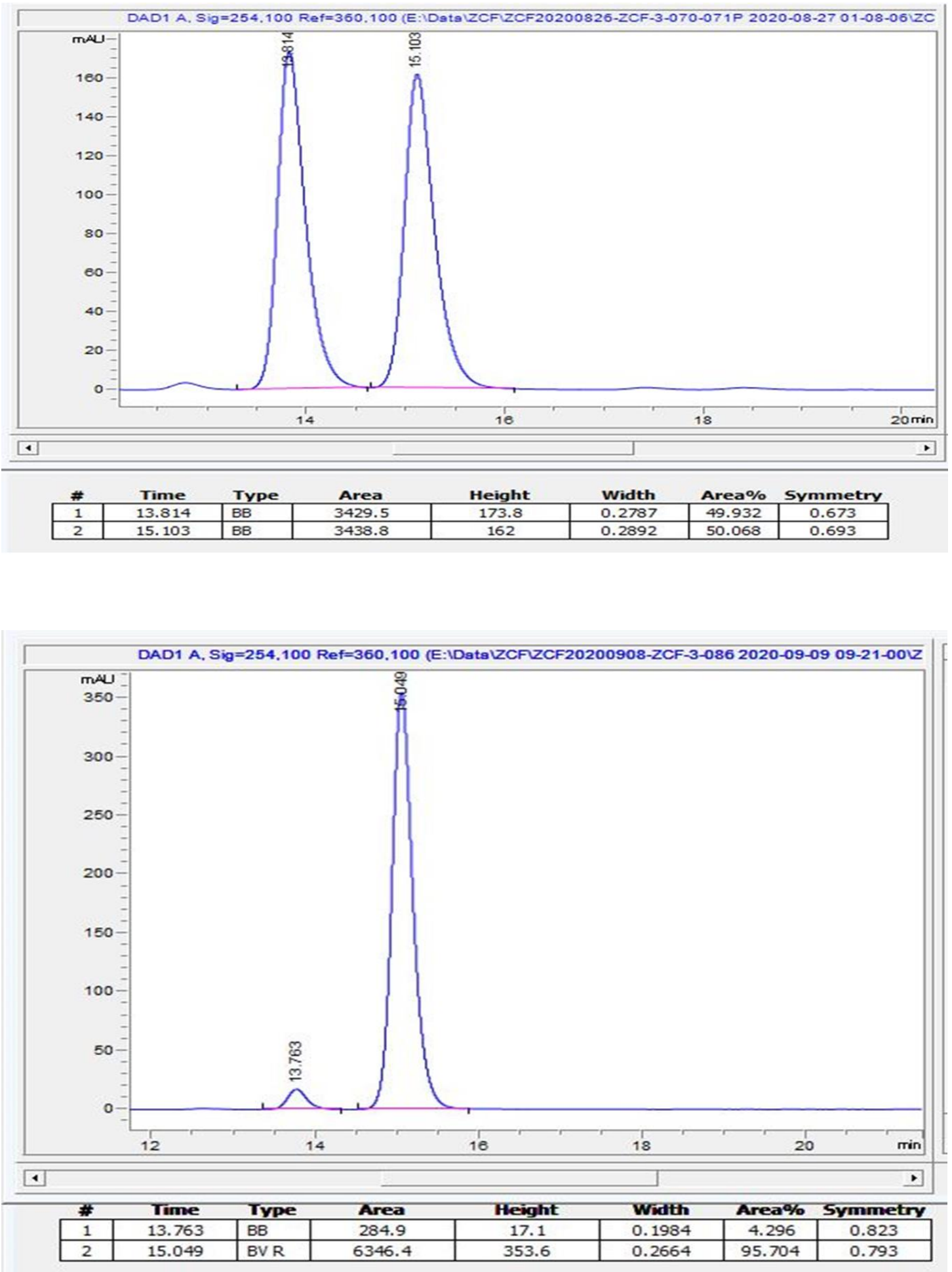
(R)-2-methyl-2-(m-tolyl)-1,2-dihydroquinoline ((R)-1f)<smiles>Cc1cccc([C@]2(C)C=Cc3ccccc3N2)c1</smiles>
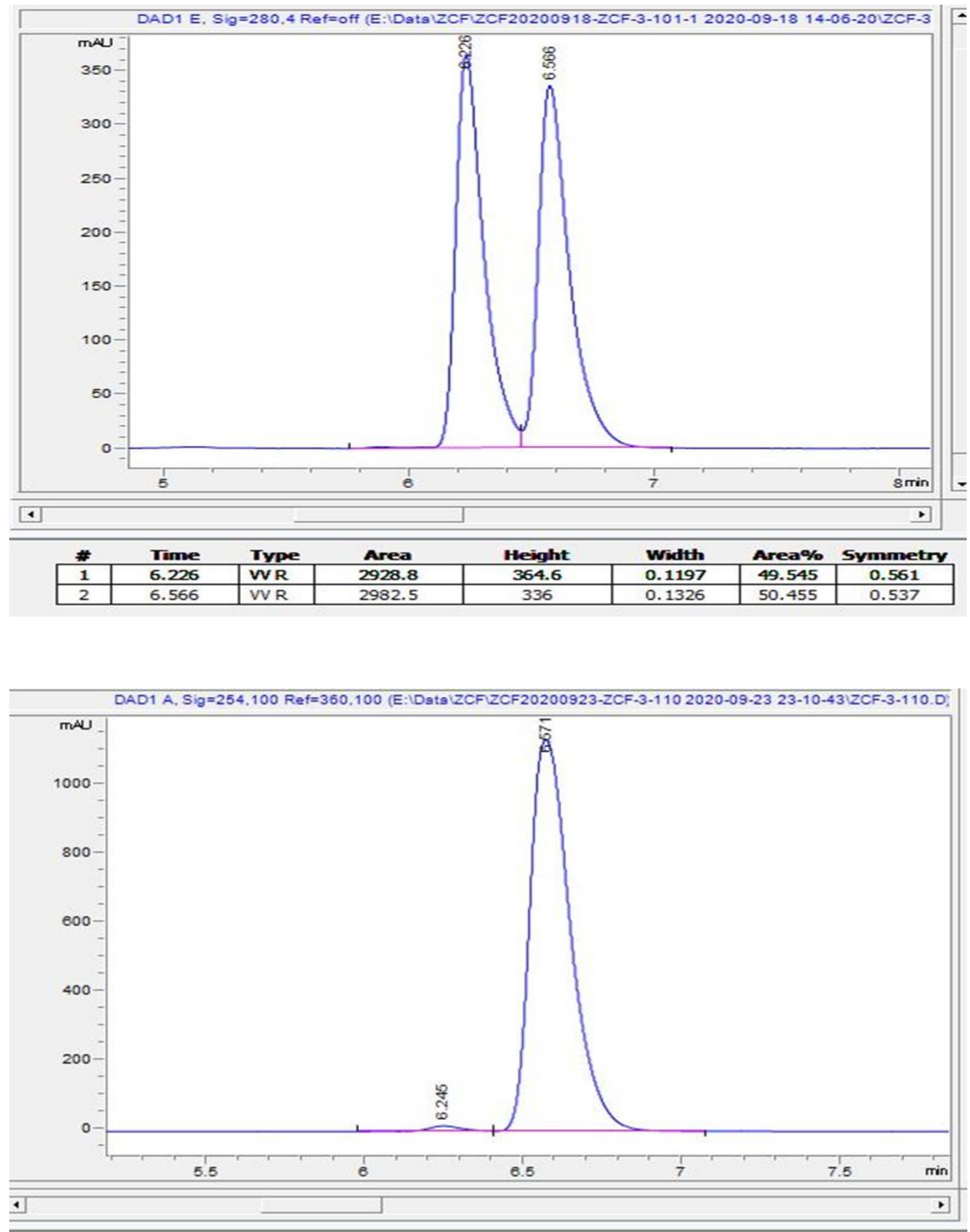

\begin{tabular}{|c|c|l|c|c|c|c|c|} 
\# & Time & \multicolumn{1}{c}{ Type } & Area & Height & Width & \multicolumn{1}{c}{ Area\% Symmetry } \\
\hline 1 & 6.245 & VVR & 115.8 & 15.5 & 0.107 & 1.139 & 0.774 \\
\hline 2 & 6.571 & VB & 10051.3 & 1136.6 & 0.1087 & 98.861 & 0.602 \\
\hline
\end{tabular}


(S)-6-bromo-2-methyl-2-(m-tolyl)-1,2-dihydroquinoline ((S)-2f)<smiles>Cc1cccc([C@]2(C)C=Cc3cc(Br)ccc3N2)c1</smiles>
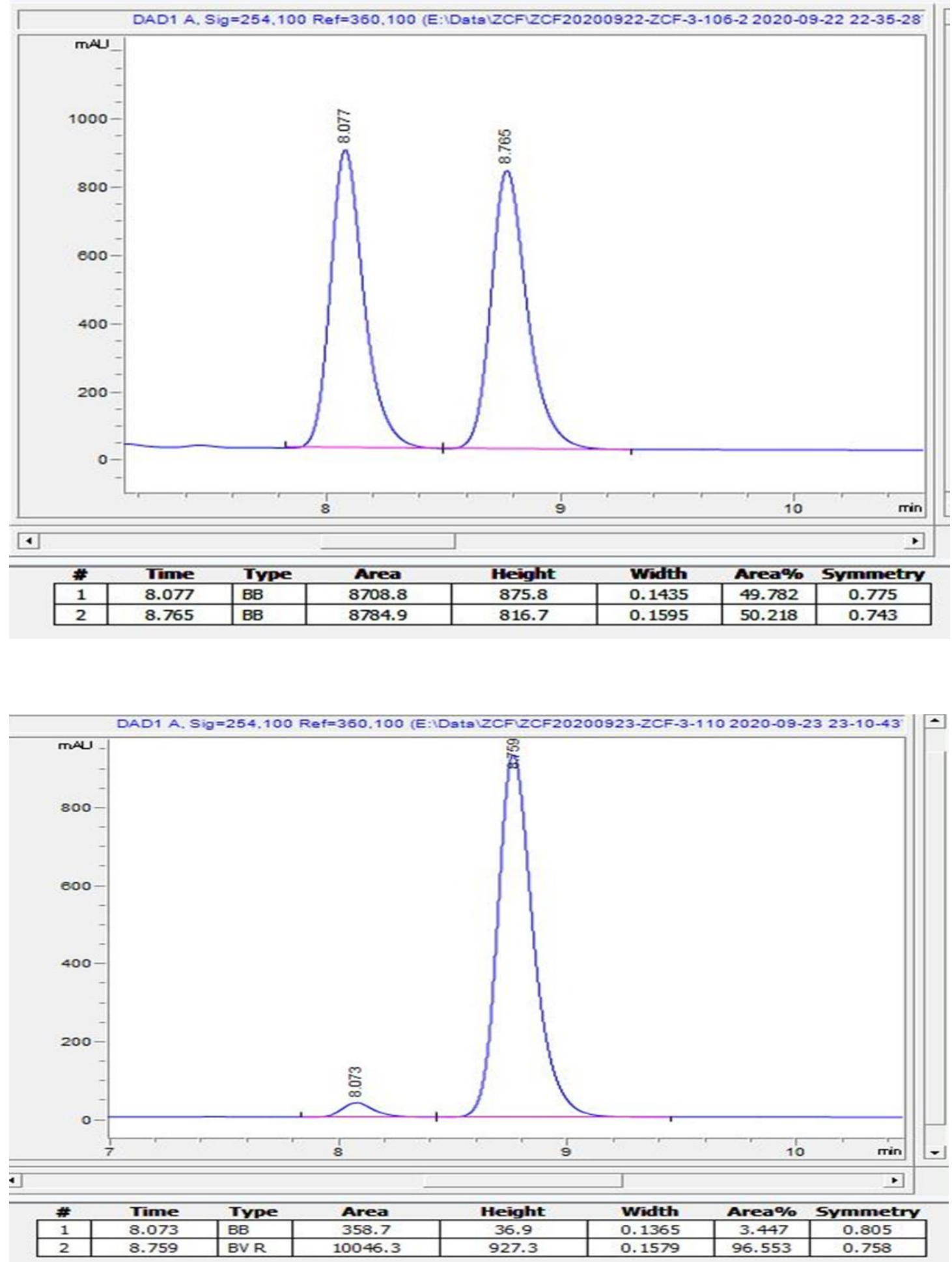
(R)-2-(3-methoxyphenyl)-2-methyl-1,2-dihydroquinoline $((R)-\mathbf{1 g})$<smiles>COc1cccc([C@]2(C)C=Cc3ccccc3N2)c1</smiles>
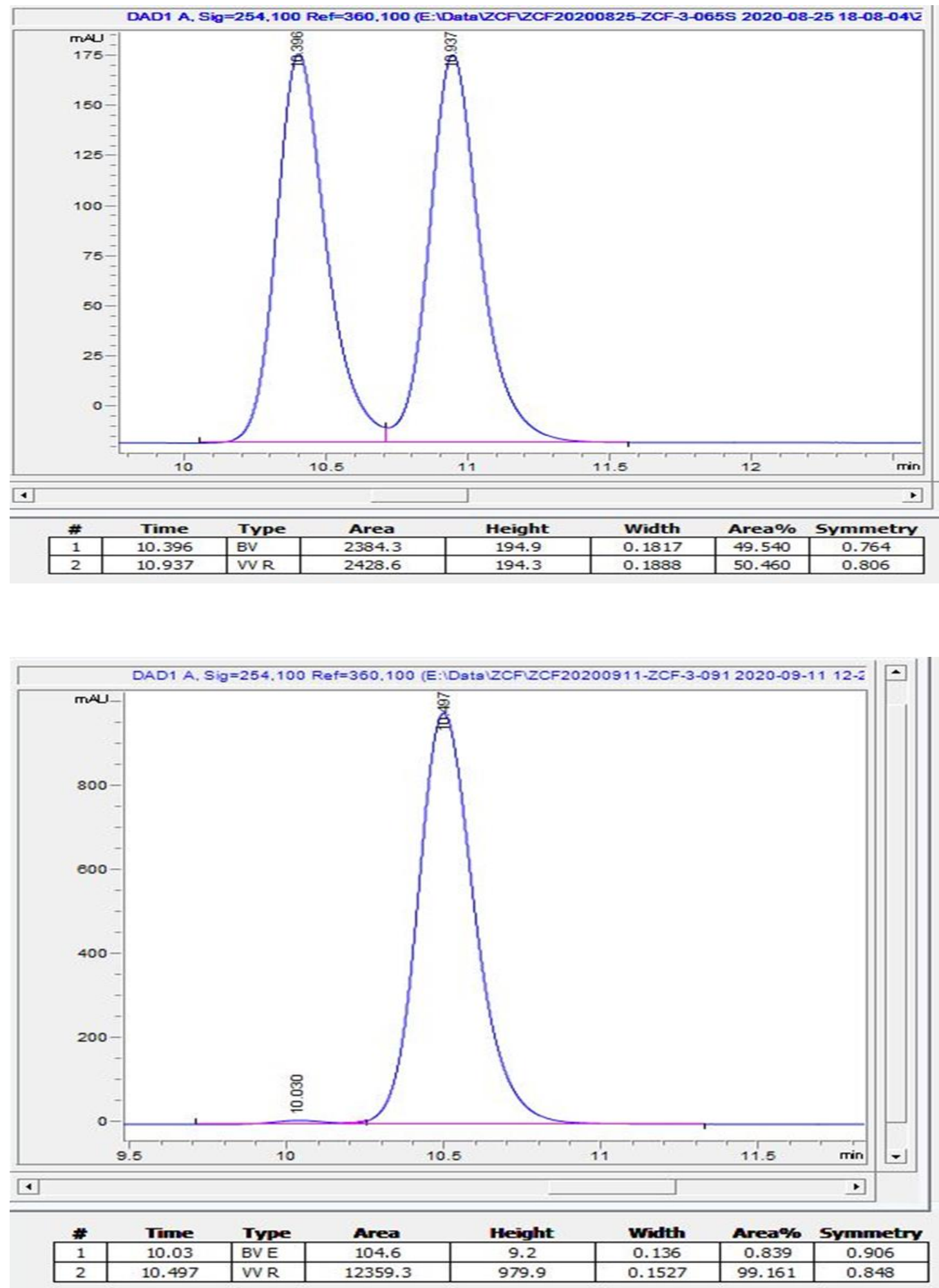
(S)-6-bromo-2-(3-methoxyphenyl)-2-methyl-1,2-dihydroquinoline ((S)-2g)<smiles>COc1cccc([C@]2(C)C=Cc3cc(Br)ccc3N2)c1</smiles>
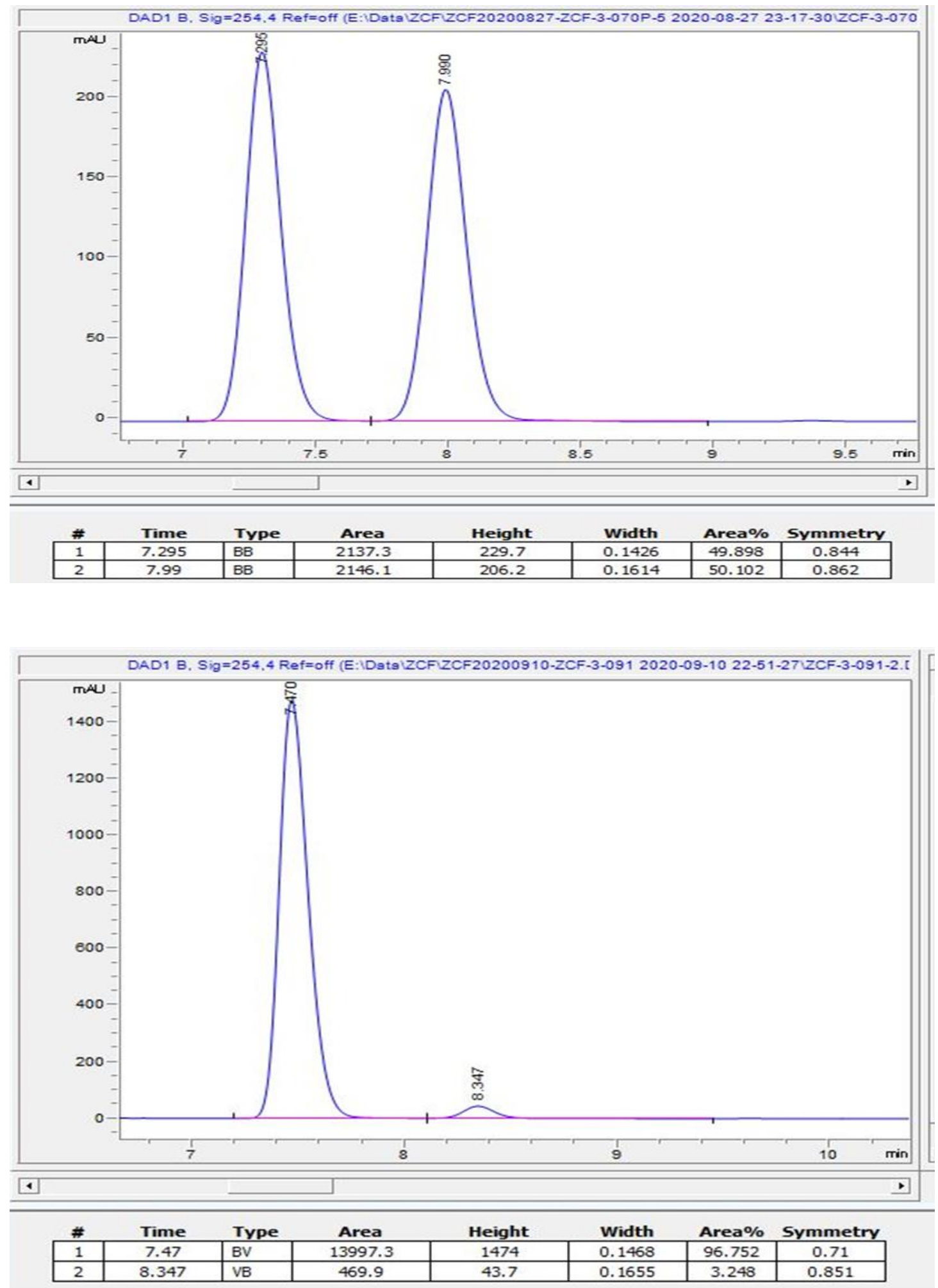
$(R)$-2-(3,5-dimethoxyphenyl)-2-methyl-1,2-dihydroquinoline $((R)-\mathbf{1 h})$<smiles>COc1cc(OC)cc([C@]2(C)C=Cc3ccccc3N2)c1</smiles>
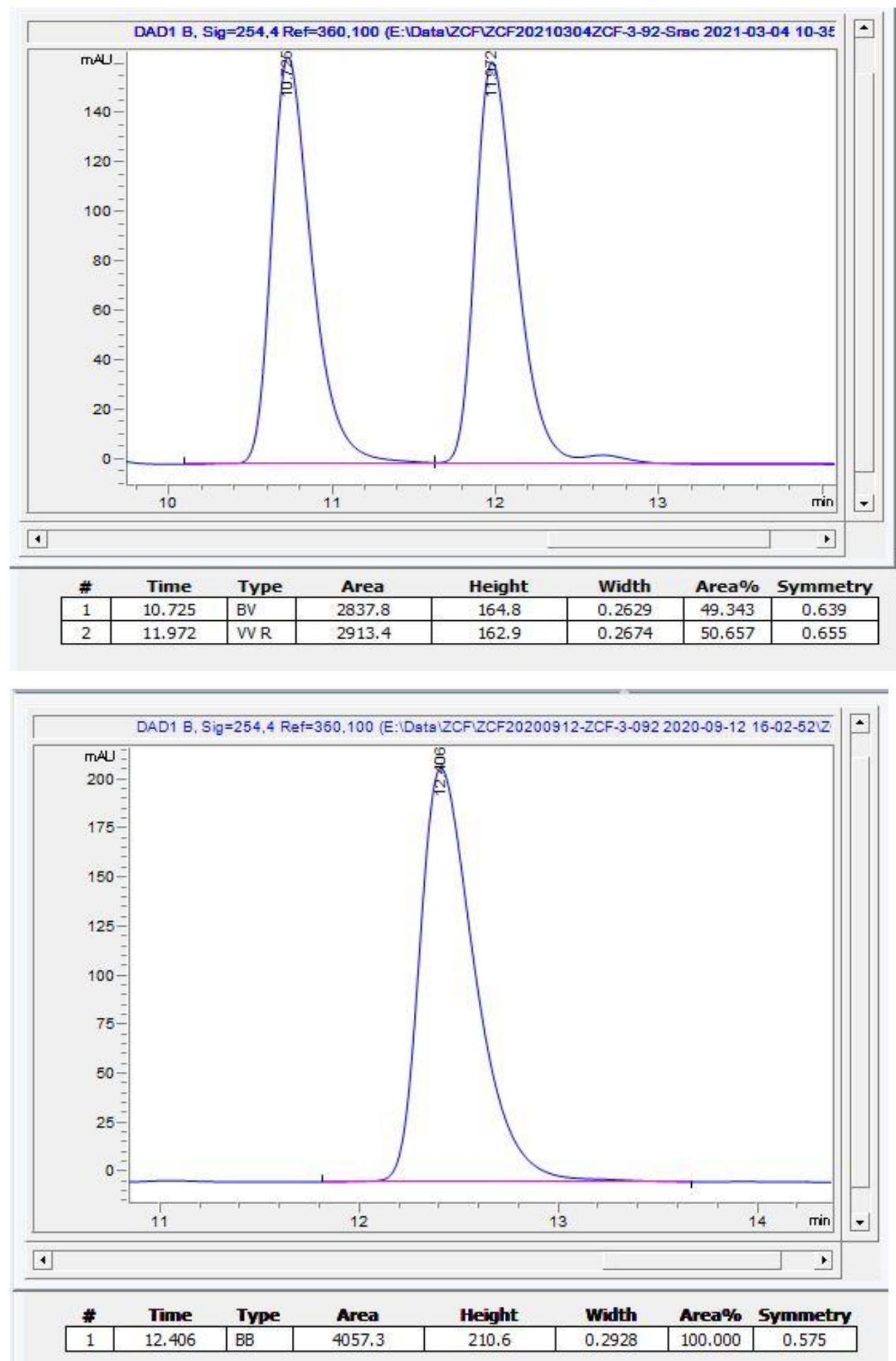
(S)-6-bromo-2-(3,5-dimethoxyphenyl)-2-methyl-1,2-dihydroquinoline ((S)-2h)<smiles>COc1cc(OC)cc([C@]2(C)C=Cc3cc(Br)ccc3N2)c1</smiles>
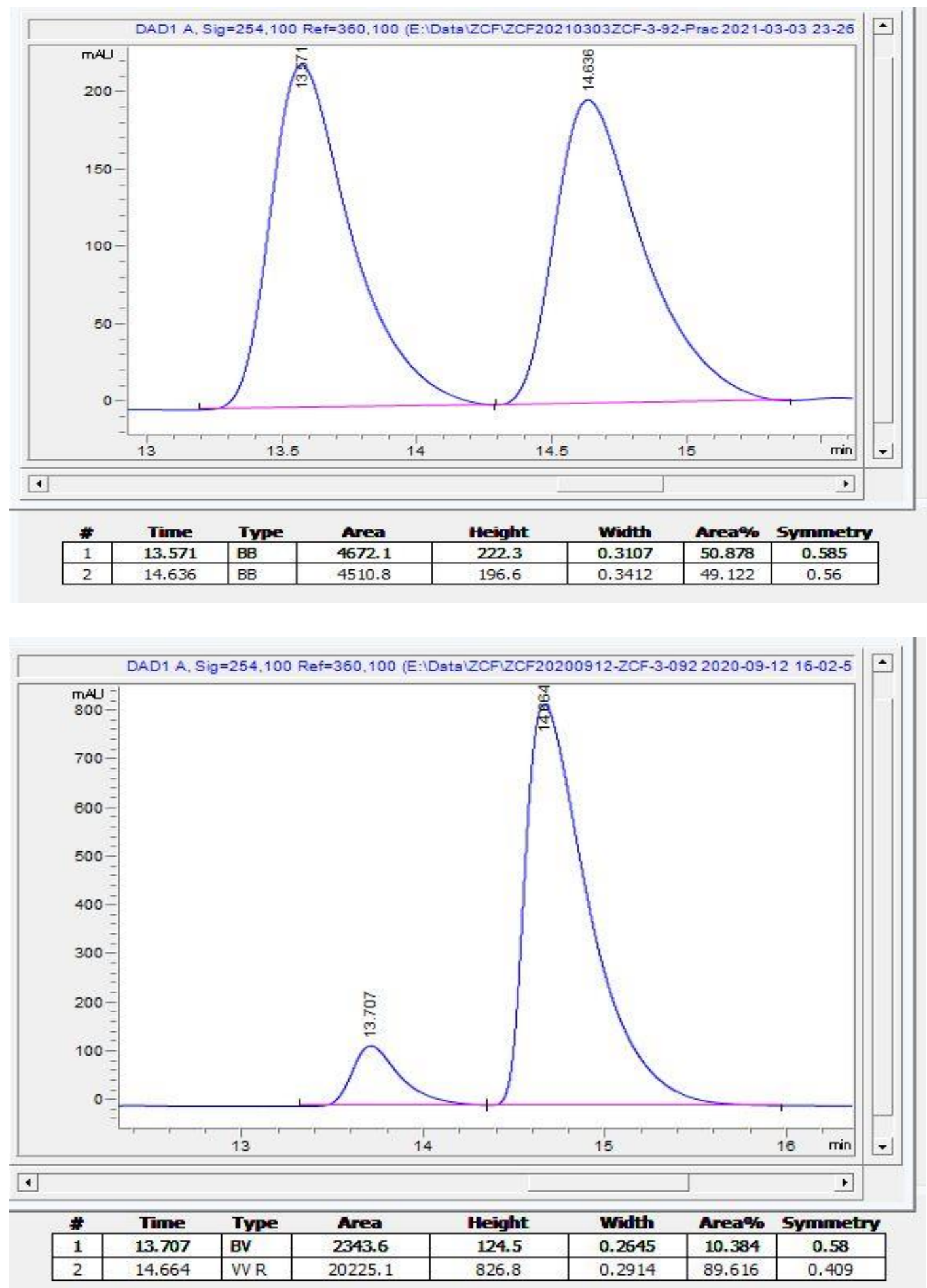
(R)-2-(benzo[d][1,3]dioxol-5-yl)-2-methyl-1,2-dihydroquinoline $((R)-1 \mathbf{i})$<smiles>CC1(c2ccc3c(c2)OCO3)C=Cc2ccccc2N1</smiles>
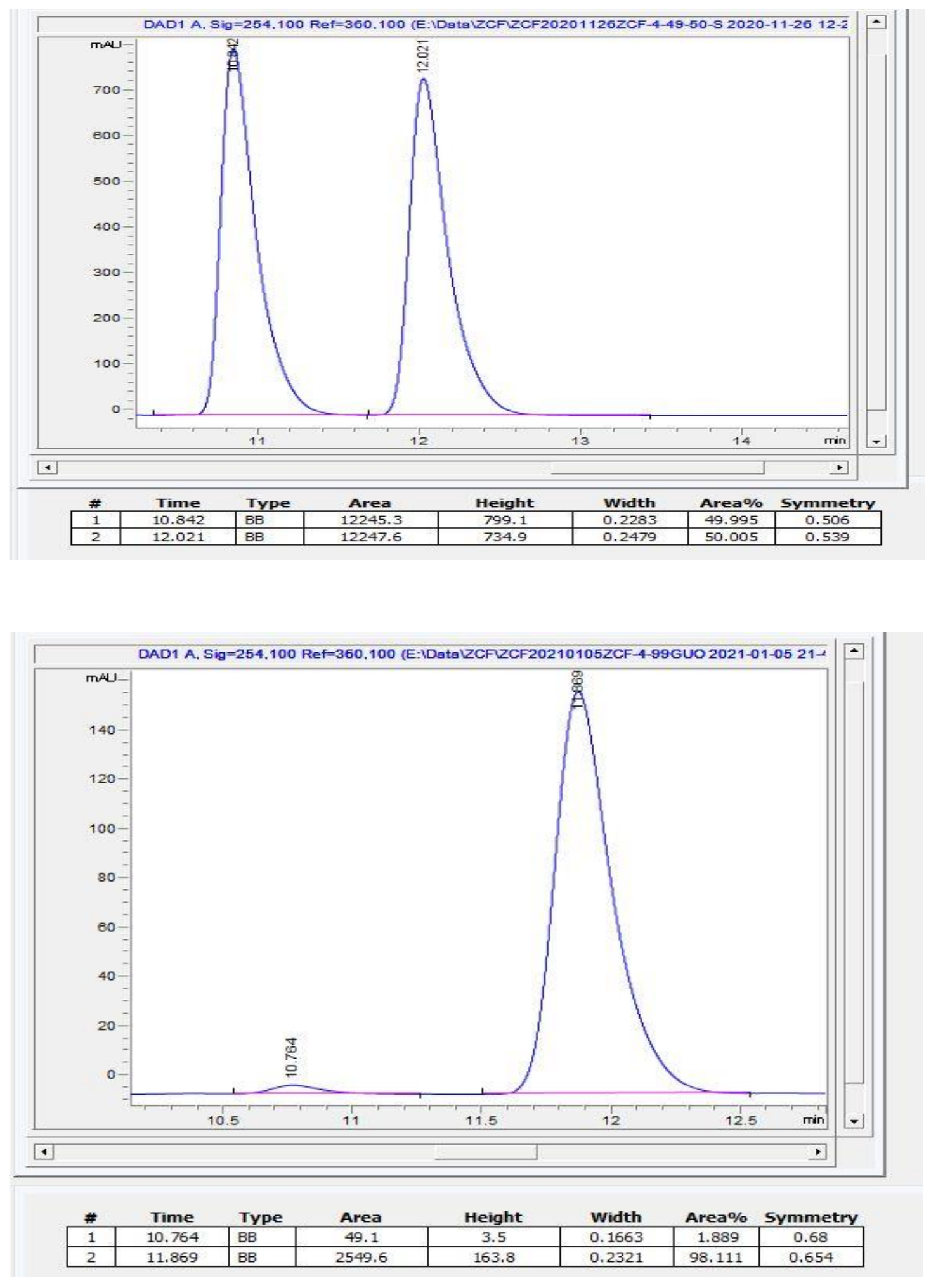
(S)-2-(benzo[d][1,3]dioxol-5-yl)-6-bromo-2-methyl-1,2-dihydroquinoline ((S)-2i)<smiles>C[C@]1(c2ccc3c(c2)OCO3)C=Cc2cc(Br)ccc2N1</smiles>
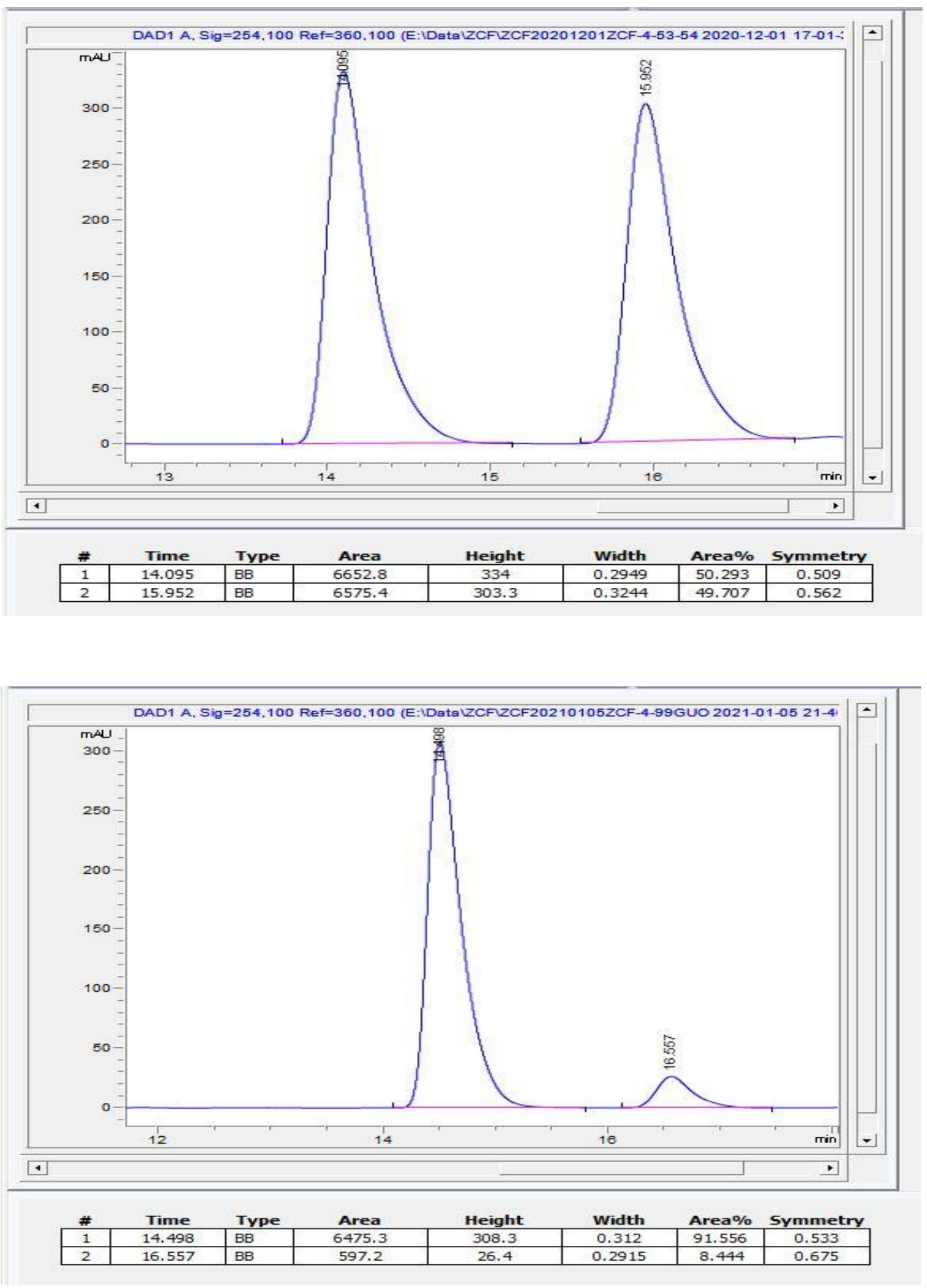
$(R)$-2-methyl-2-(thiophen-3-yl)-1,2-dihydroquinoline $((R)-\mathbf{1 j})$<smiles>CC1(c2ccsc2)C=Cc2ccccc2N1</smiles>

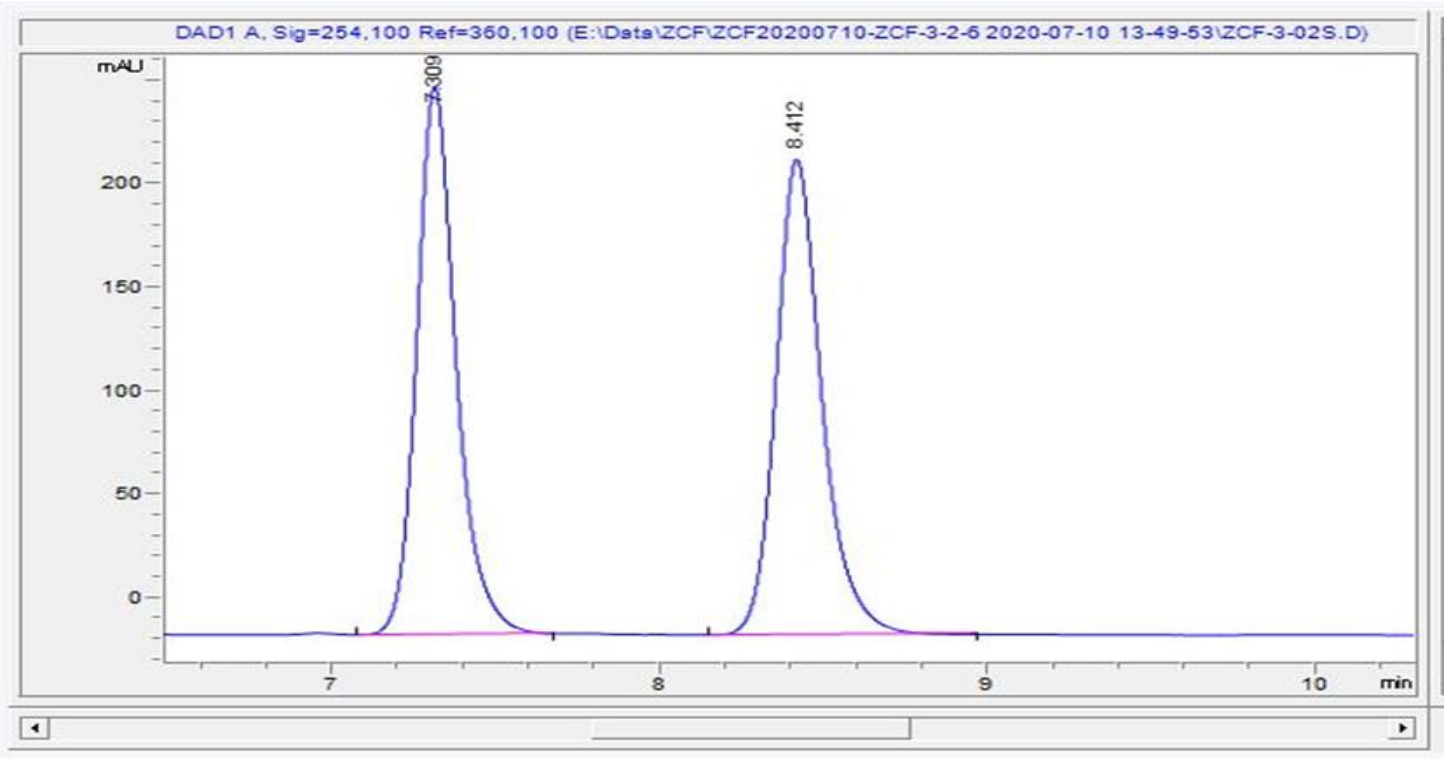

\begin{tabular}{|c|c|l|c|c|c|c|c|} 
\# & \multicolumn{1}{c}{ Time } & \multicolumn{1}{c}{ Type } & Area & Height & Width & Area\% $\%$ Symmetry \\
\hline 1 & 7.309 & BB & 2214.6 & 265.6 & 0.1263 & 49.681 & 0.792 \\
\hline 2 & 8.412 & BB & 2243 & 230.6 & 0.146 & 50.319 & 0.778 \\
\hline
\end{tabular}

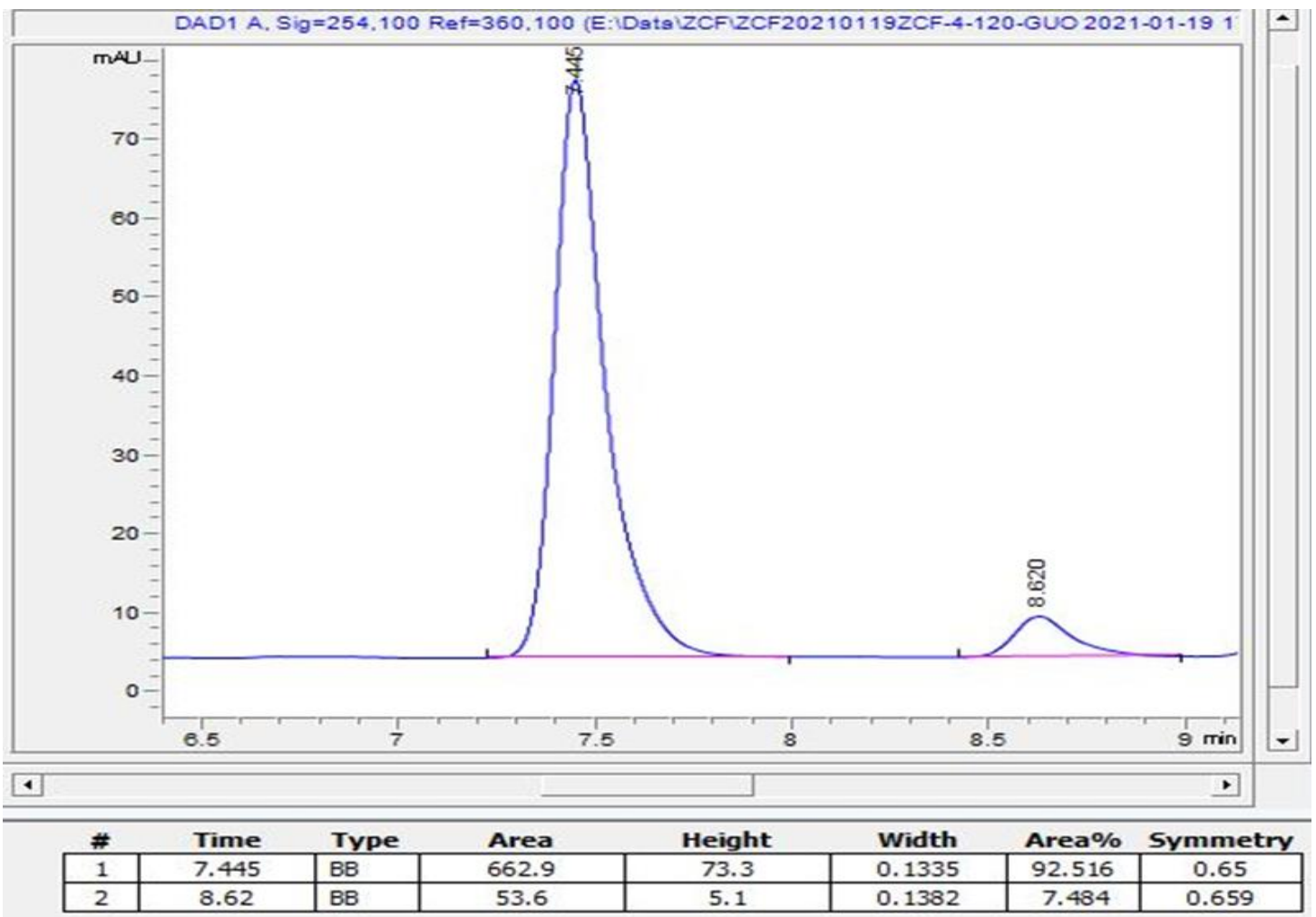


(S)-6-bromo-2-methyl-2-(thiophen-3-yl)-1,2-dihydroquinoline ((S)-2j)<smiles>C[C@]1(c2ccsc2)C=Cc2cc(Br)ccc2N1</smiles>
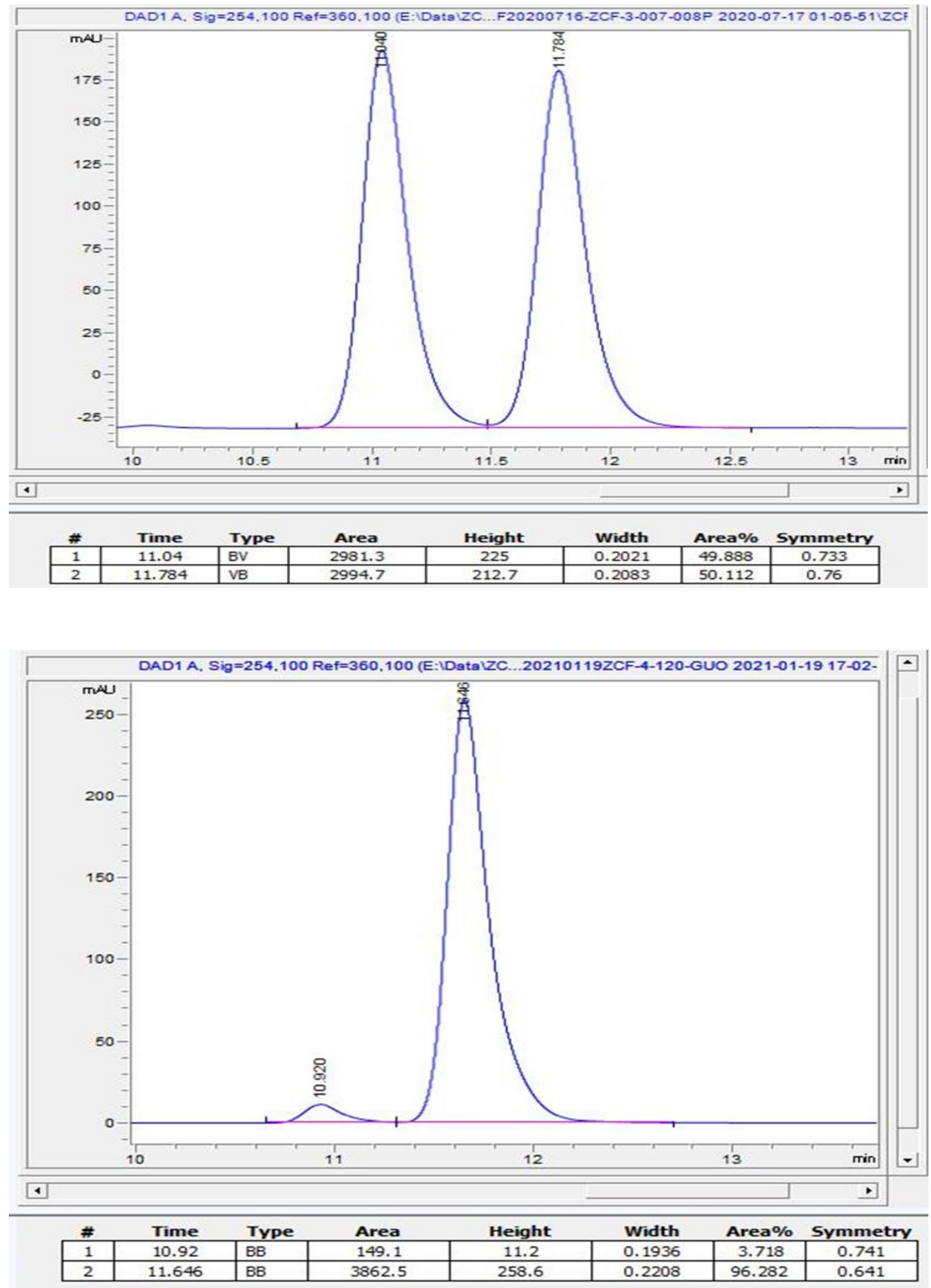
(R)-2-(furan-3-yl)-2-methyl-1,2-dihydroquinoline $((R)-1 \mathbf{k})$<smiles>C[C@]1(c2ccoc2)C=Cc2ccccc2N1</smiles>

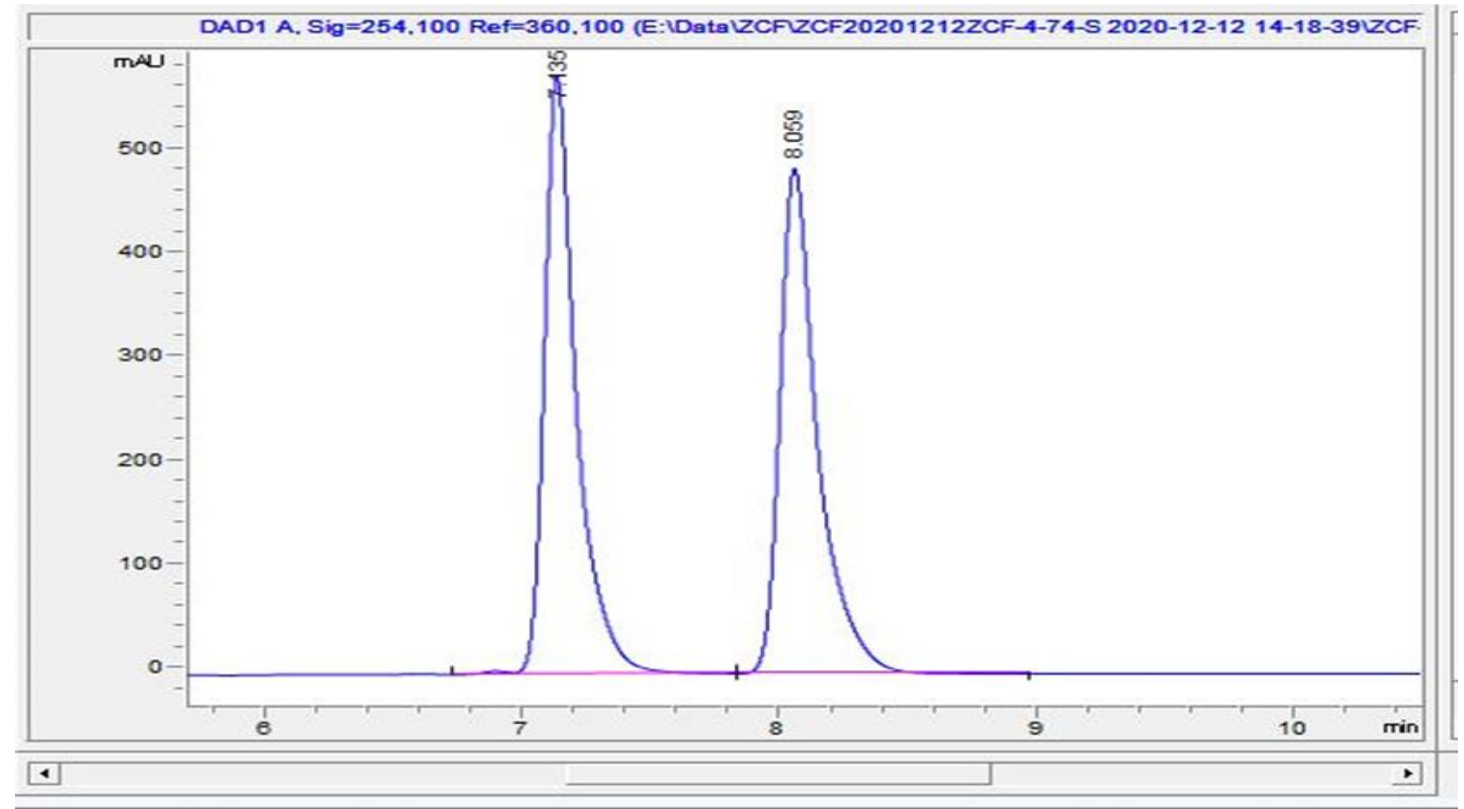

\begin{tabular}{|c|c|l|c|c|c|c|c|}
\multicolumn{2}{l}{ \# } & Time & \multicolumn{1}{c}{ Type } & Area & \multicolumn{1}{c}{ Height } & Width & \multicolumn{1}{c}{ Area\% $\%$ Symmetry } \\
\hline 1 & 7.135 & VB R & 4992.4 & 576.9 & 0.127 & 50.162 & 0.612 \\
\hline 2 & 8.059 & BB & 4960.1 & 486.9 & 0.1499 & 49.838 & 0.577 \\
\hline
\end{tabular}

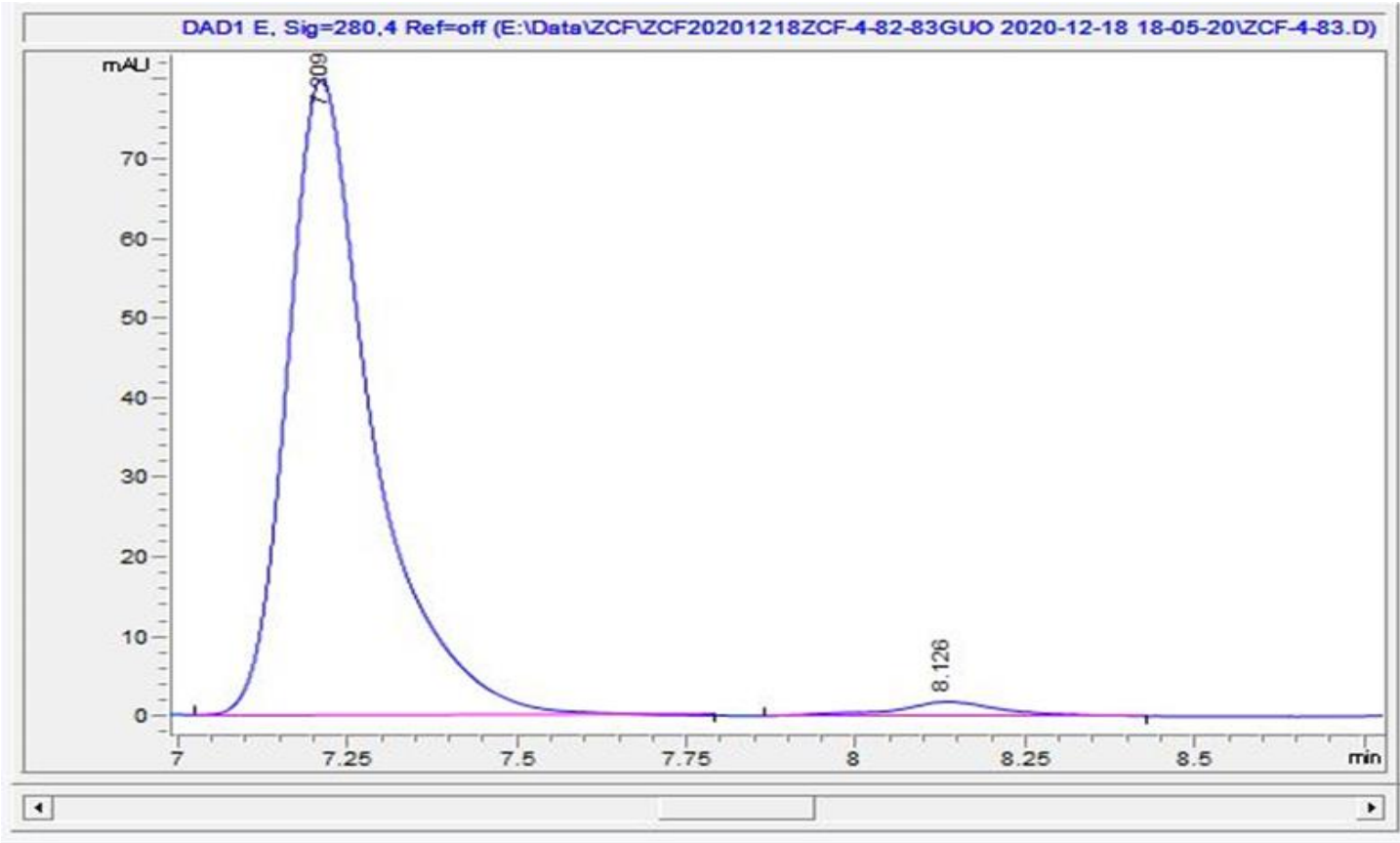

\begin{tabular}{|l|c|l|c|c|c|c|c|} 
\# & \multicolumn{1}{c}{ Time } & \multicolumn{1}{c}{ Type } & Area & Height & Width & \multicolumn{1}{c|}{ Area\% Symmetry } \\
\hline 1 & 7.209 & VB & 699.9 & 80 & 0.1291 & 97.233 & 0.608 \\
\hline 2 & 8.126 & BB & 19.9 & 1.8 & 0.1317 & 2.767 & 0.873 \\
\hline
\end{tabular}


(S)-6-bromo-2-(furan-3-yl)-2-methyl-1,2-dihydroquinoline ((S)-2k)<smiles>C[C@]1(c2ccoc2)C=Cc2cc(Br)ccc2N1</smiles>

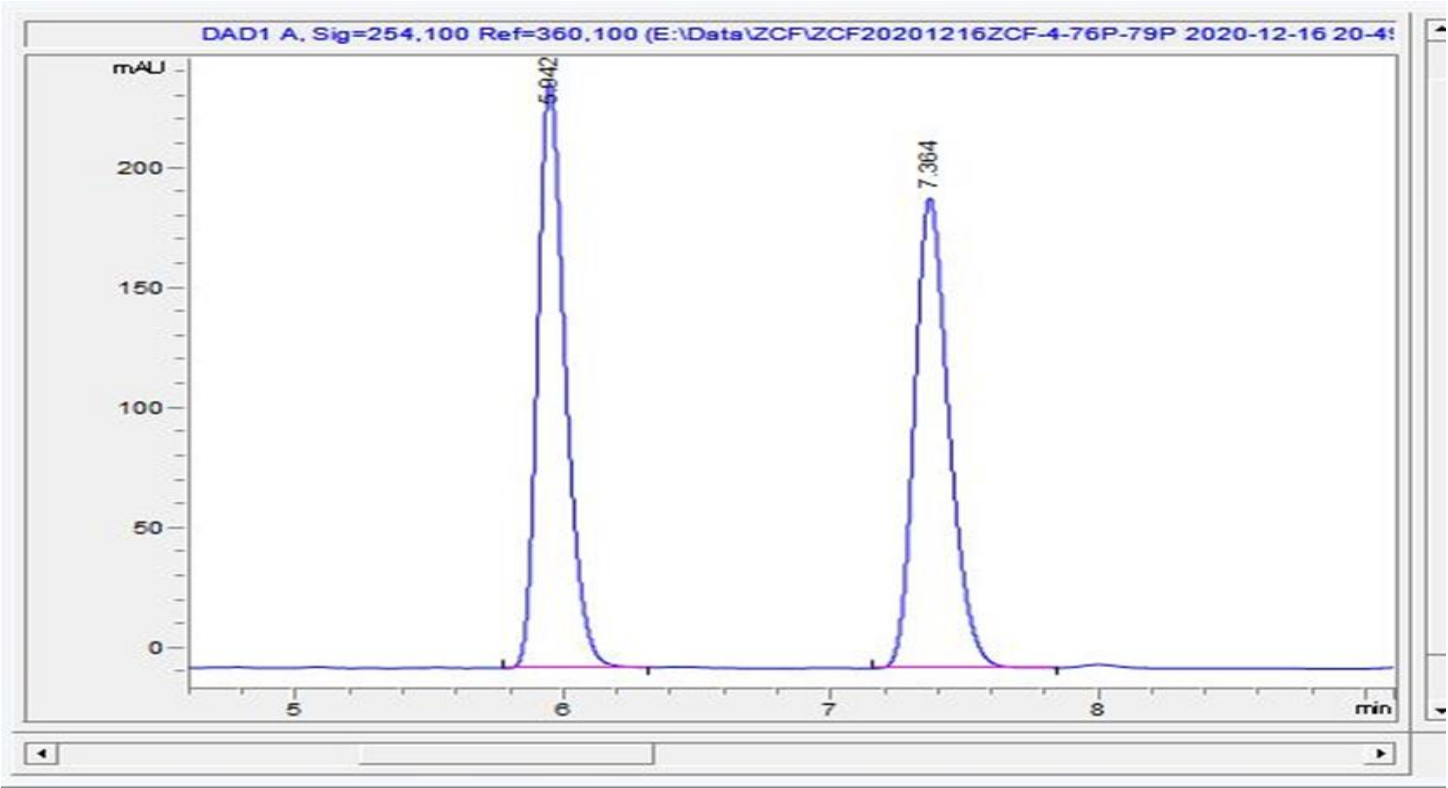

\begin{tabular}{|c|c|l|c|c|c|c|c|}
\hline & Time & \multicolumn{1}{c}{ Type } & Area & Height & Width & \multicolumn{1}{c}{ Area\% Symmetry } \\
\hline 1 & 5.942 & BB & 1751.5 & 243.7 & 0.1098 & 49.982 & 0.704 \\
\hline 2 & 7.364 & BB & 1752.7 & 196 & 0.1368 & 50.018 & 0.745 \\
\hline
\end{tabular}

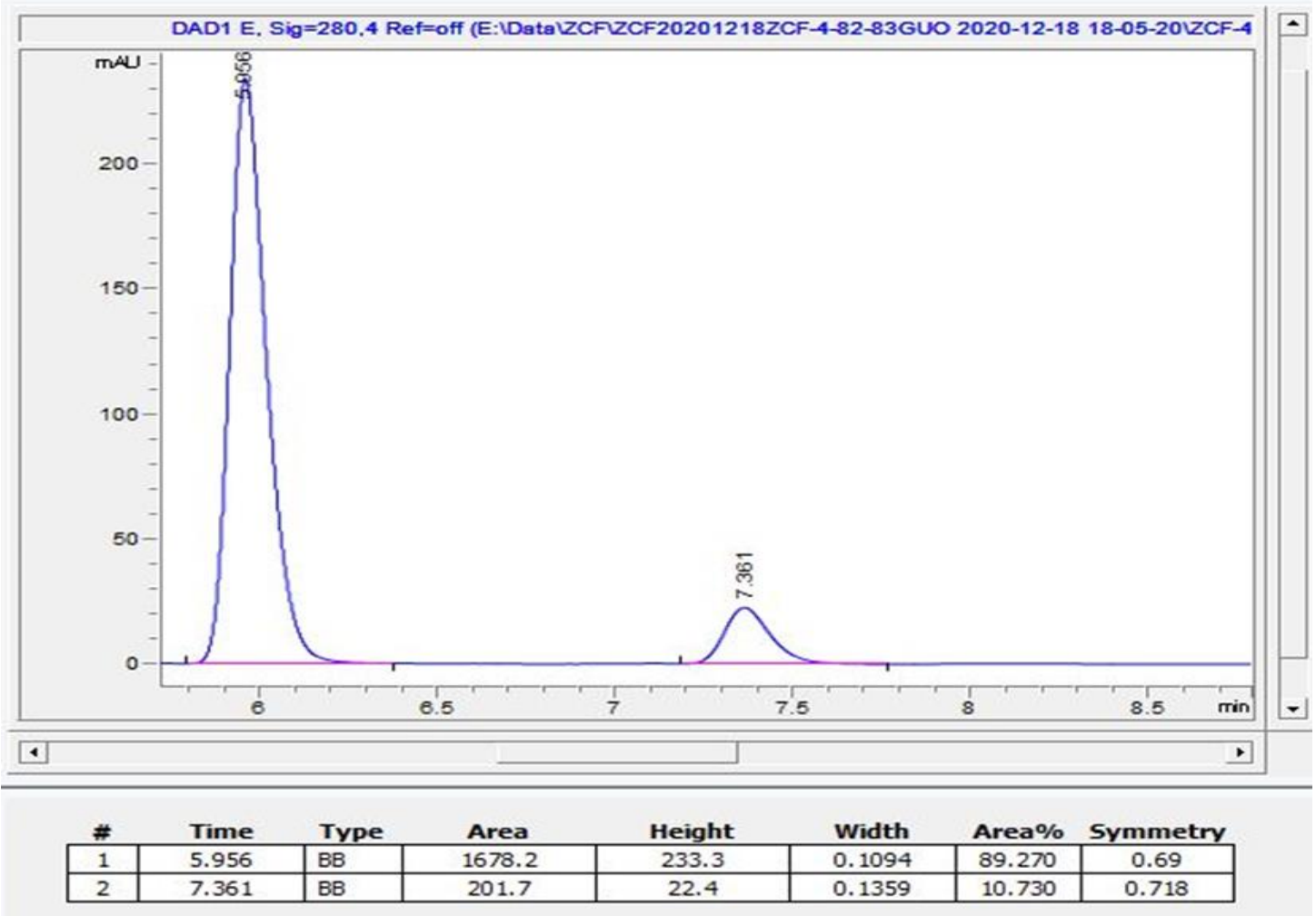


$(R)$-2-ethyl-2-phenyl-1,2-dihydroquinoline $((R)$-11)<smiles>CC[C@@]1(c2ccccc2)C=Cc2ccccc2N1</smiles>

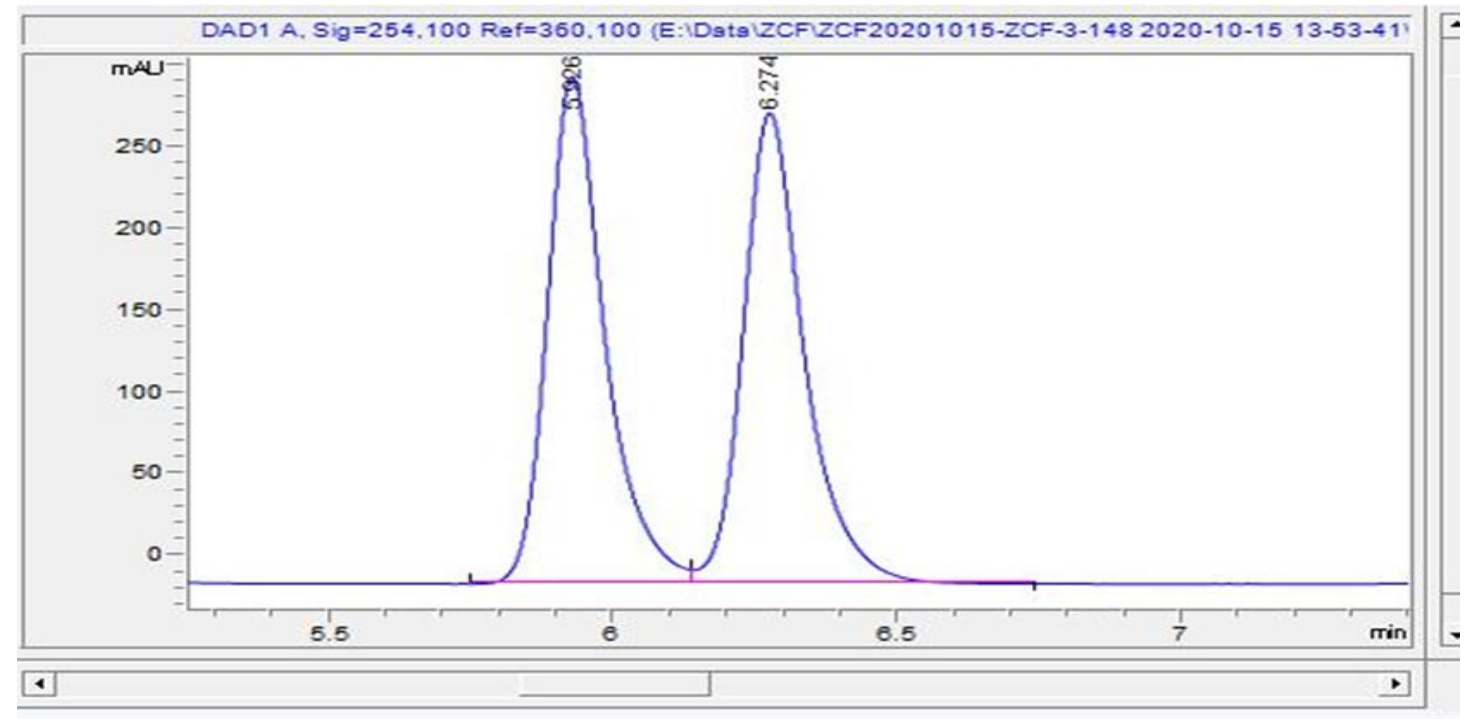

\begin{tabular}{|c|c|l|l|c|c|c|c|} 
\# & Time & \multicolumn{1}{c}{ Type } & \multicolumn{1}{c}{ Area } & Height & \multicolumn{1}{c}{ Width } & \multicolumn{1}{c}{ Area\% Symmetry } \\
\hline 1 & 5.926 & BV & 2191.2 & 310.9 & 0.1052 & 50.164 & 0.704 \\
\hline 2 & 6.274 & VV R & 2176.9 & 288.6 & 0.113 & 49.836 & 0.714 \\
\hline
\end{tabular}

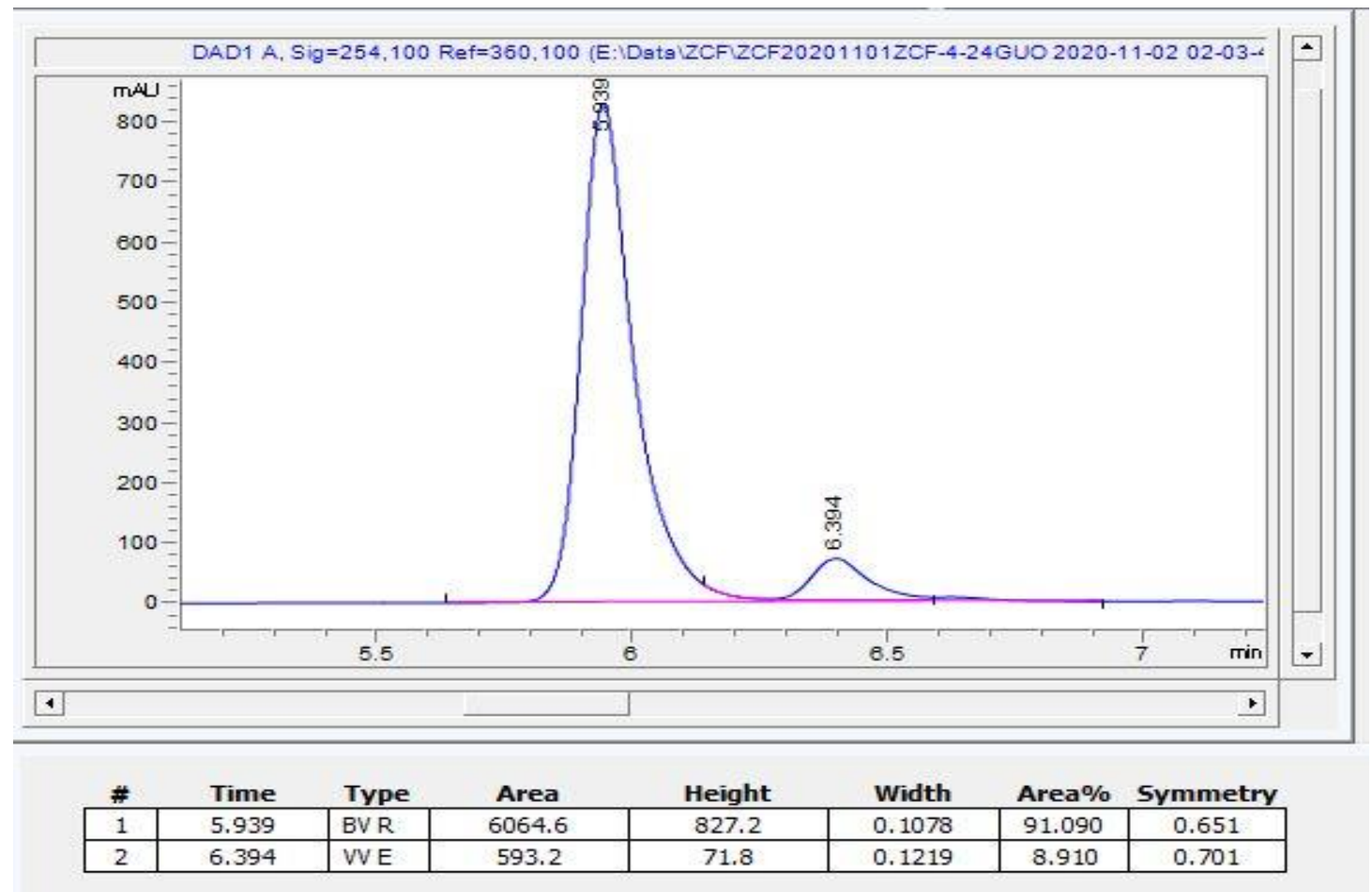


(S)-6-bromo-2-ethyl-2-phenyl-1,2-dihydroquinoline ((S)-2l)<smiles>CC[C@@]1(c2ccccc2)C=Cc2cc(Br)ccc2N1</smiles>

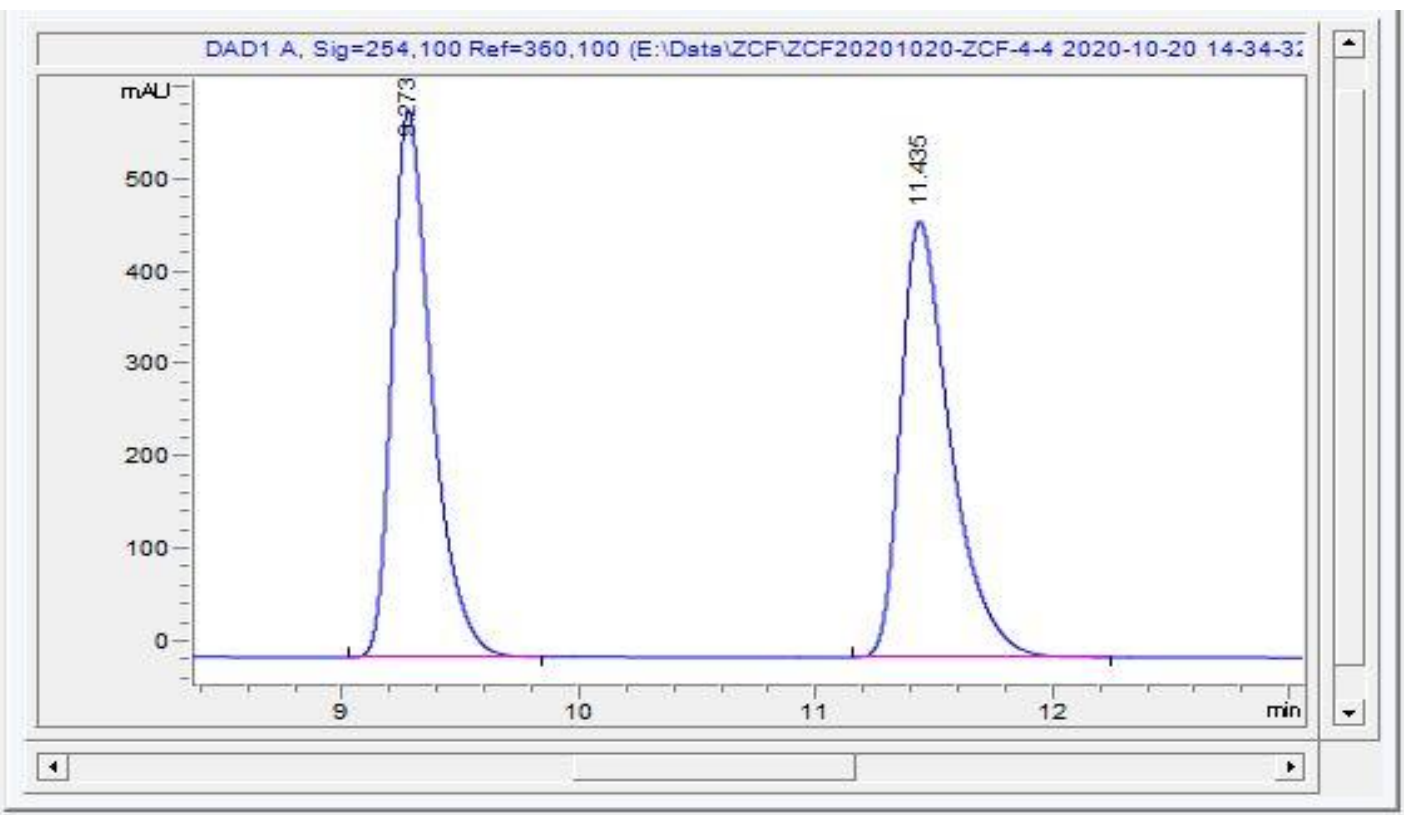

\begin{tabular}{|l|c|l|c|c|c|c|c|} 
\# & \multicolumn{1}{c}{ Time } & \multicolumn{1}{c}{ Type } & Area & Height & \multicolumn{1}{c}{ width } & \multicolumn{2}{c}{ Area\% } \\
\hline 1 & 9.273 & BV R & 6906.4 & 595 & 0.1659 & 49.926 & 0.641 \\
\hline 2 & 11.435 & BV R & 6926.9 & 474.9 & 0.2017 & 50.074 & 0.576 \\
\hline
\end{tabular}

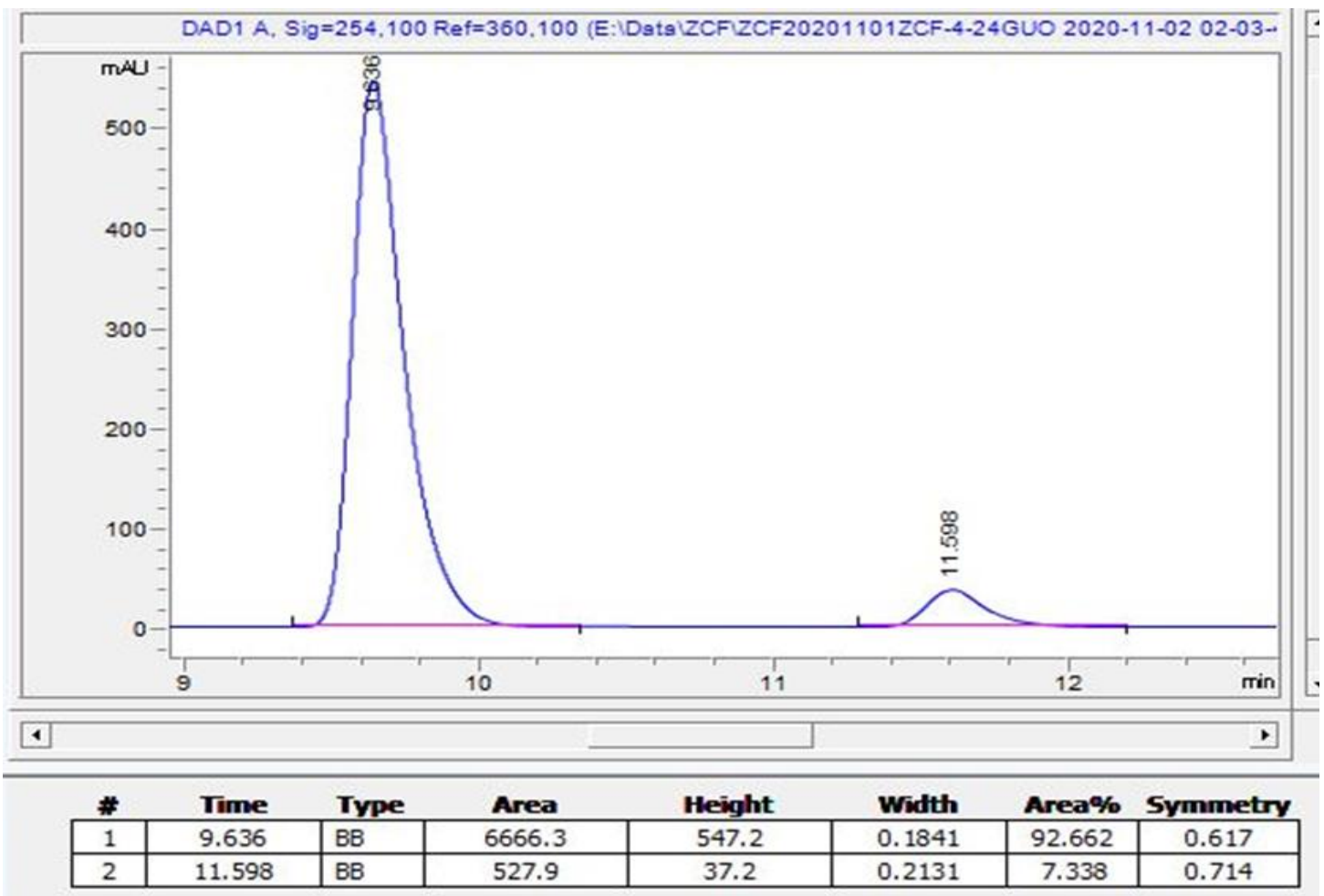


(R)-2-ethyl-2-(4-methoxyphenyl)-1,2-dihydroquinoline ((R)-1 m)<smiles>CC[C@@]1(c2ccc(OC)cc2)C=Cc2ccccc2N1</smiles>

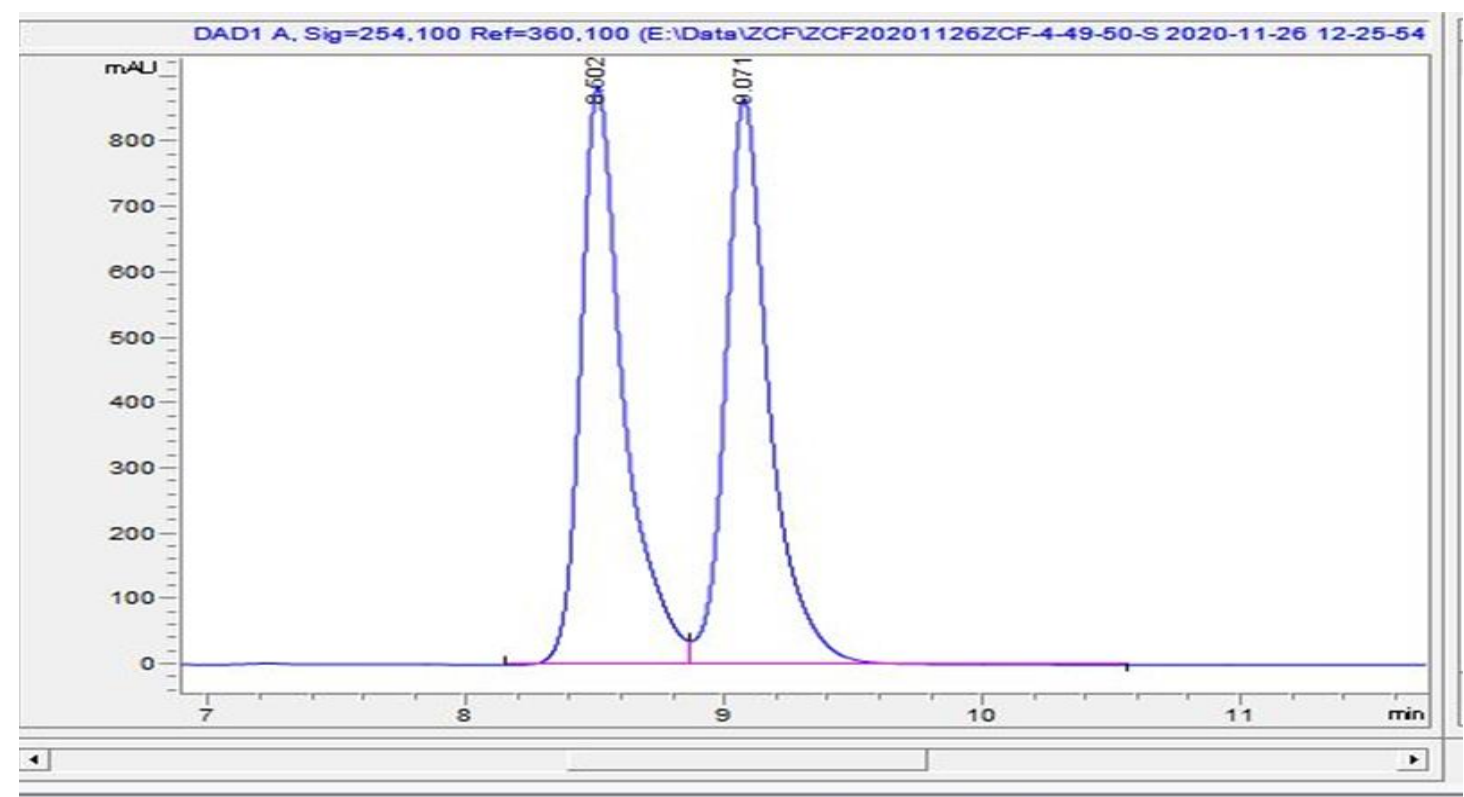

\begin{tabular}{|c|c|l|c|c|c|c|c|}
\multicolumn{1}{|l}{ Time } & \multicolumn{1}{c}{ Type } & Area & Height & Width & \multicolumn{1}{c}{ Area\% Symmetry } \\
\hline 1 & 8.502 & BV & 10334.7 & 883.5 & 0.172 & 50.235 & 0.602 \\
\hline 2 & 9.071 & VB & 10237.8 & 863.8 & 0.1753 & 49.765 & 0.69 \\
\hline
\end{tabular}

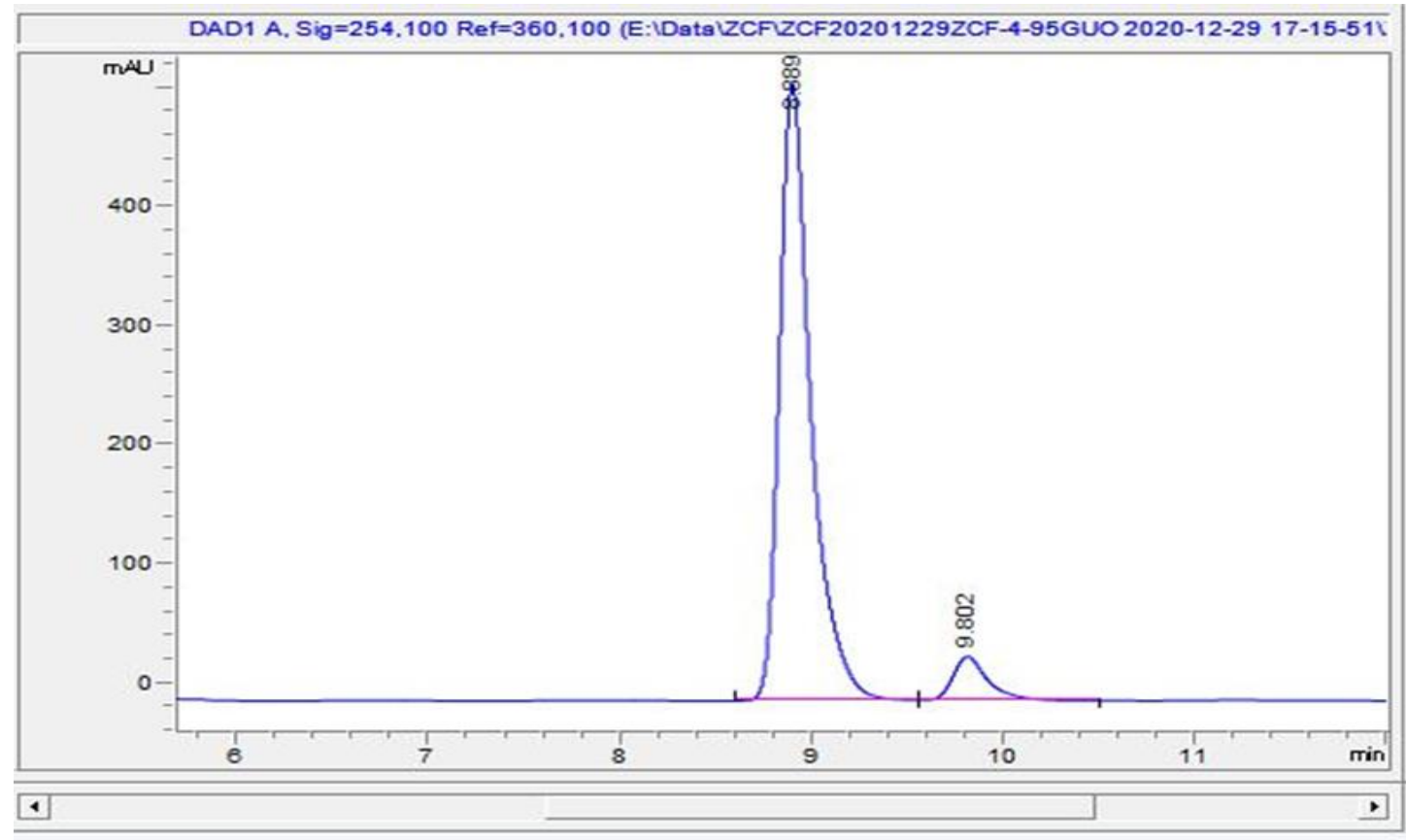

\begin{tabular}{|c|c|l|c|c|c|c|c|} 
\# & \multicolumn{1}{c}{ Time } & \multicolumn{1}{c}{ Type } & Area & Height & \multicolumn{1}{c}{ Width } & \multicolumn{1}{c}{ Area\% } & Symmetry \\
\hline 1 & 8.889 & BB & 5882.1 & 516 & 0.17 & 92.802 & 0.627 \\
\hline 2 & 9.802 & B & 456.2 & 36.5 & 0.1828 & 7.198 & 0.65 \\
\hline
\end{tabular}


(S)-6-bromo-2-ethyl-2-(4-methoxyphenyl)-1,2-dihydroquinoline ((S)-2m)<smiles>CCC(CC)(c1ccc(OC)cc1)C1C=Cc2cc(Br)ccc2N1</smiles>

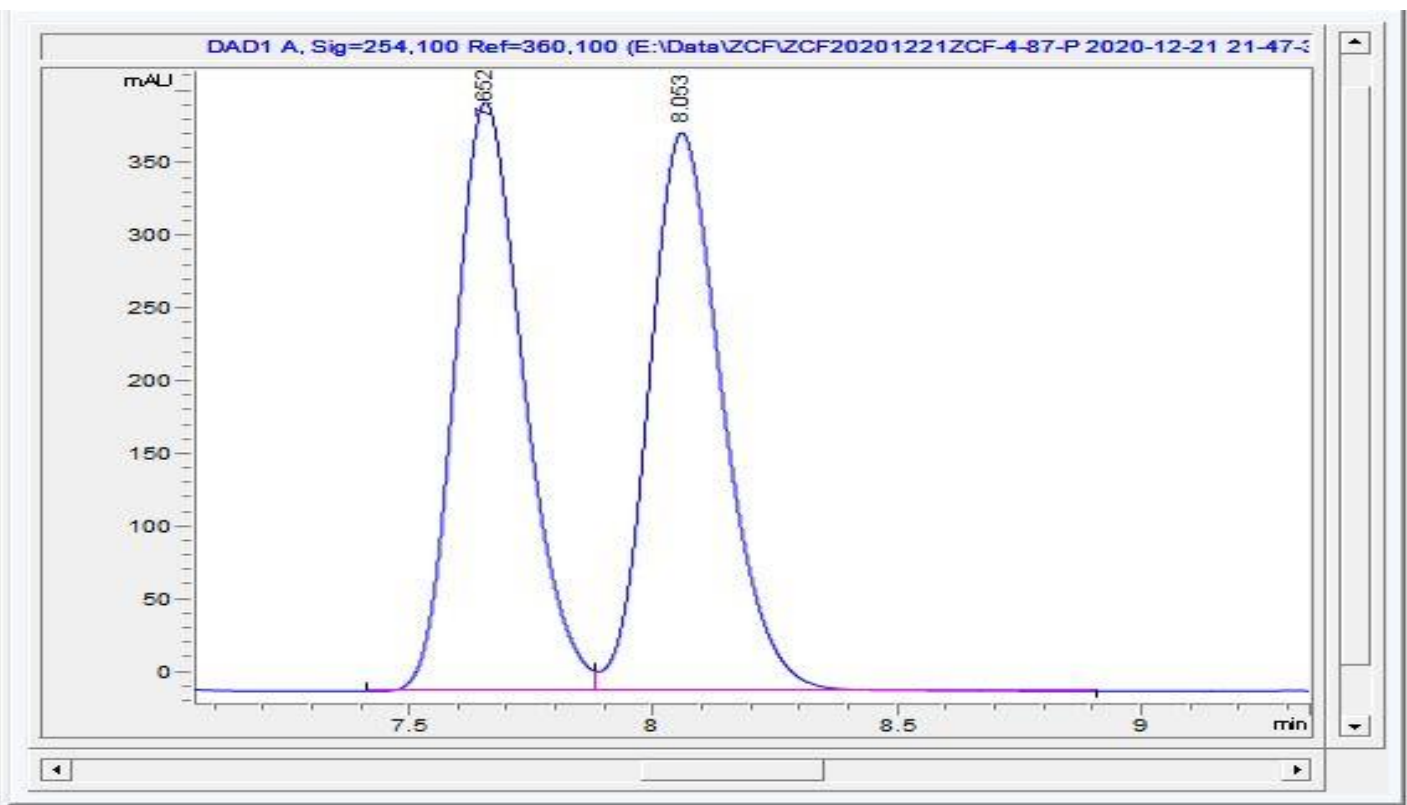

\begin{tabular}{|c|c|l|c|c|c|c|c|} 
\# & \multicolumn{1}{c}{ Time } & \multicolumn{1}{c}{ Type } & Area & Height & Width & \multicolumn{2}{c}{ Area\% Symmetry } \\
\hline 1 & 7.652 & BV & 3958.1 & 406.1 & 0.1487 & 49.639 & 0.754 \\
\hline 2 & 8.053 & VB & 4015.6 & 385.2 & 0.1601 & 50.361 & 0.754 \\
\hline
\end{tabular}

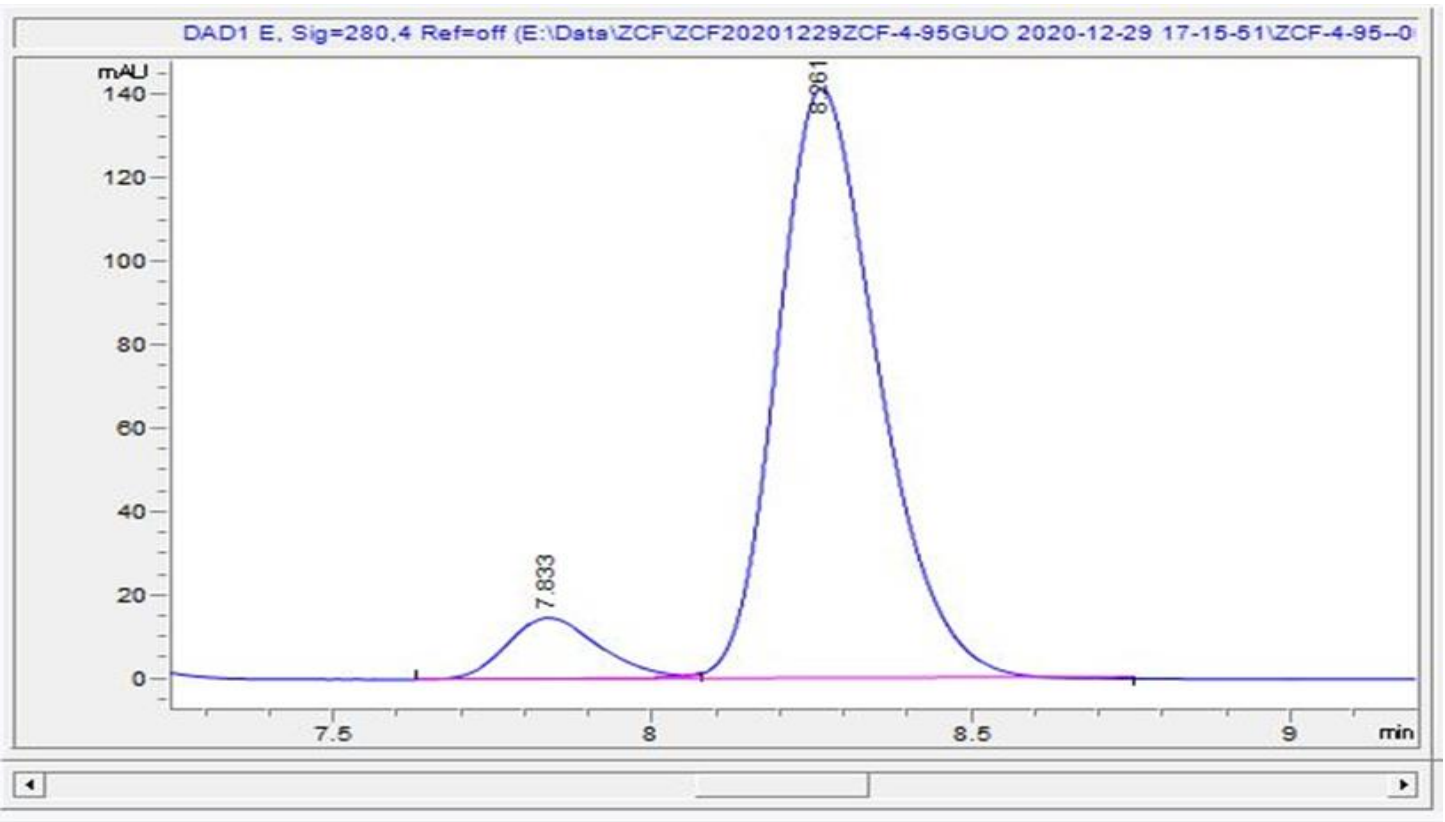

\begin{tabular}{|c|c|l|c|c|c|c|c|} 
\# & \multicolumn{1}{c}{ Time } & \multicolumn{1}{c}{ Type } & Area & Height & Width & \multicolumn{1}{c}{ Area\% $\%$ Symmetry } \\
\hline 1 & 7.833 & BVE & 149.1 & 14.7 & 0.1432 & 8.729 & 0.759 \\
\hline 2 & 8.261 & VB R & 1559.5 & 141.5 & 0.1695 & 91.271 & 1.172 \\
\hline
\end{tabular}


(R)-2-ethyl-2-(3-methoxyphenyl)-1,2-dihydroquinoline ((R)-1n)<smiles>CC[C@]1(c2cccc(OC)c2)C=Cc2ccccc2N1</smiles>
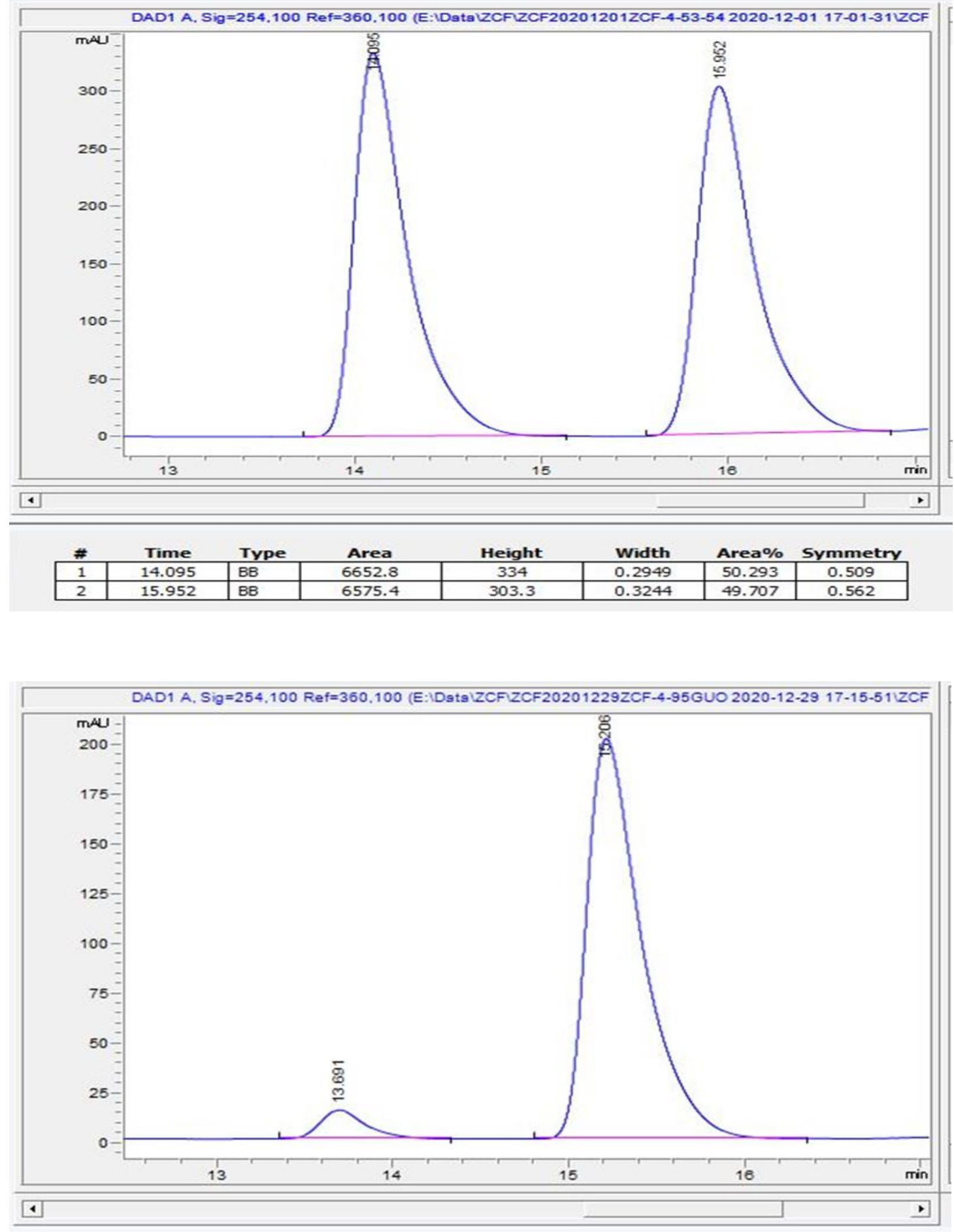

\begin{tabular}{|c|c|c|c|c|c|c|c|}
\hline$\#$ & Time & Type & Area & Height & Width & Area $\%$ & Symmetry \\
\hline 1 & 13.691 & $B B$ & 260.6 & 14.5 & 0.2432 & 5.569 & 0.687 \\
\hline 2 & 15.206 & $B B$ & 4419.3 & 201.9 & 0.3209 & 94.431 & 0.516 \\
\hline
\end{tabular}


(S)-6-bromo-2-ethyl-2-(3-methoxyphenyl)-1,2-dihydroquinoline ((S)-2n)<smiles>CC[C@](C)(c1cccc(OC)c1)C1C=Cc2cc(Br)ccc2N1</smiles>

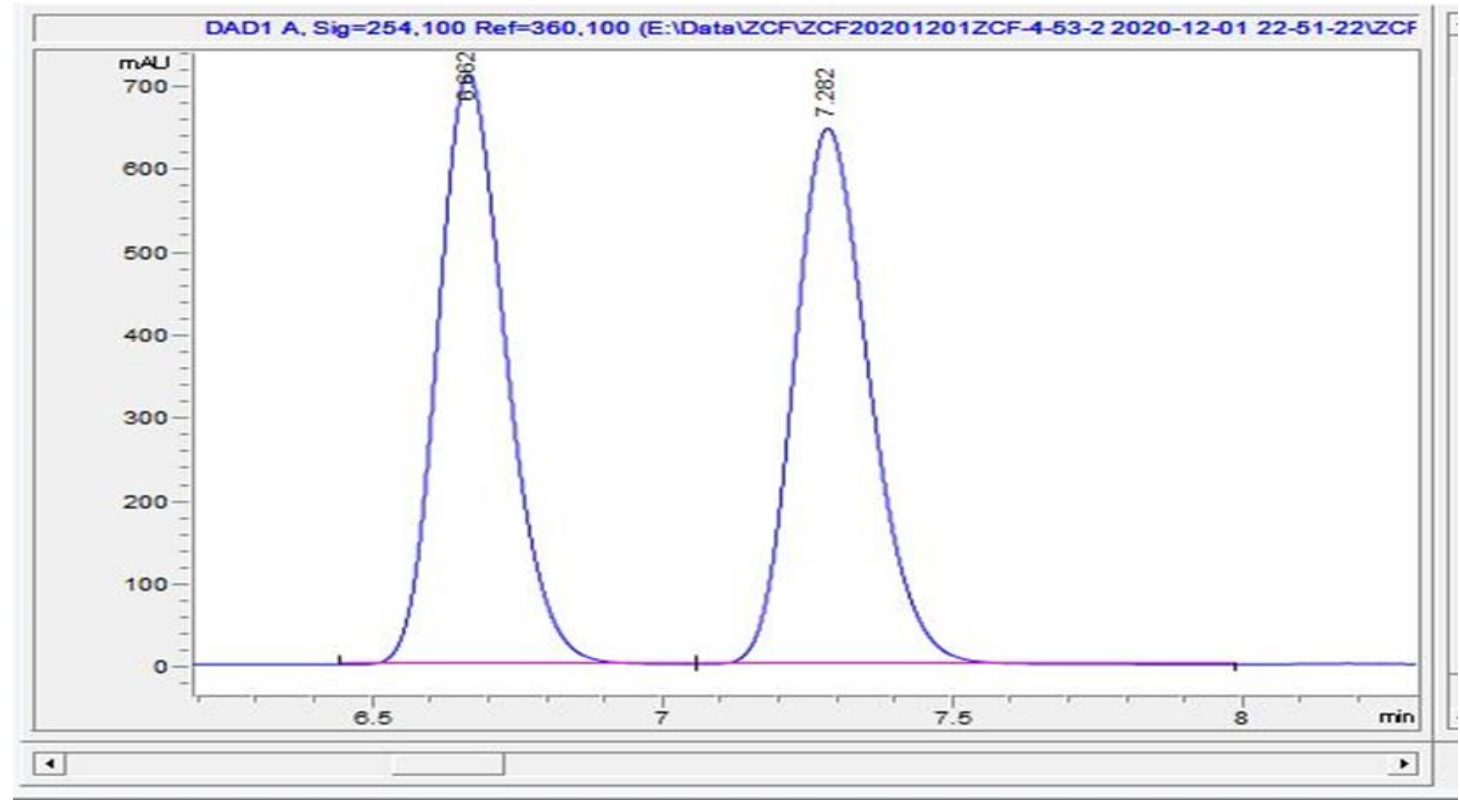

\begin{tabular}{|c|c|c|c|c|c|c|c|}
\hline \# & Time & Type & Area & Height & Width & Area $\%$ & Symmetry \\
\hline 1 & 6.662 & BB & 5813.3 & 712.8 & 0.1262 & 49.928 & 0.774 \\
\hline 2 & 7.282 & BB & 5830.1 & 647.7 & 0.1395 & 50.072 & 0.772 \\
\hline
\end{tabular}

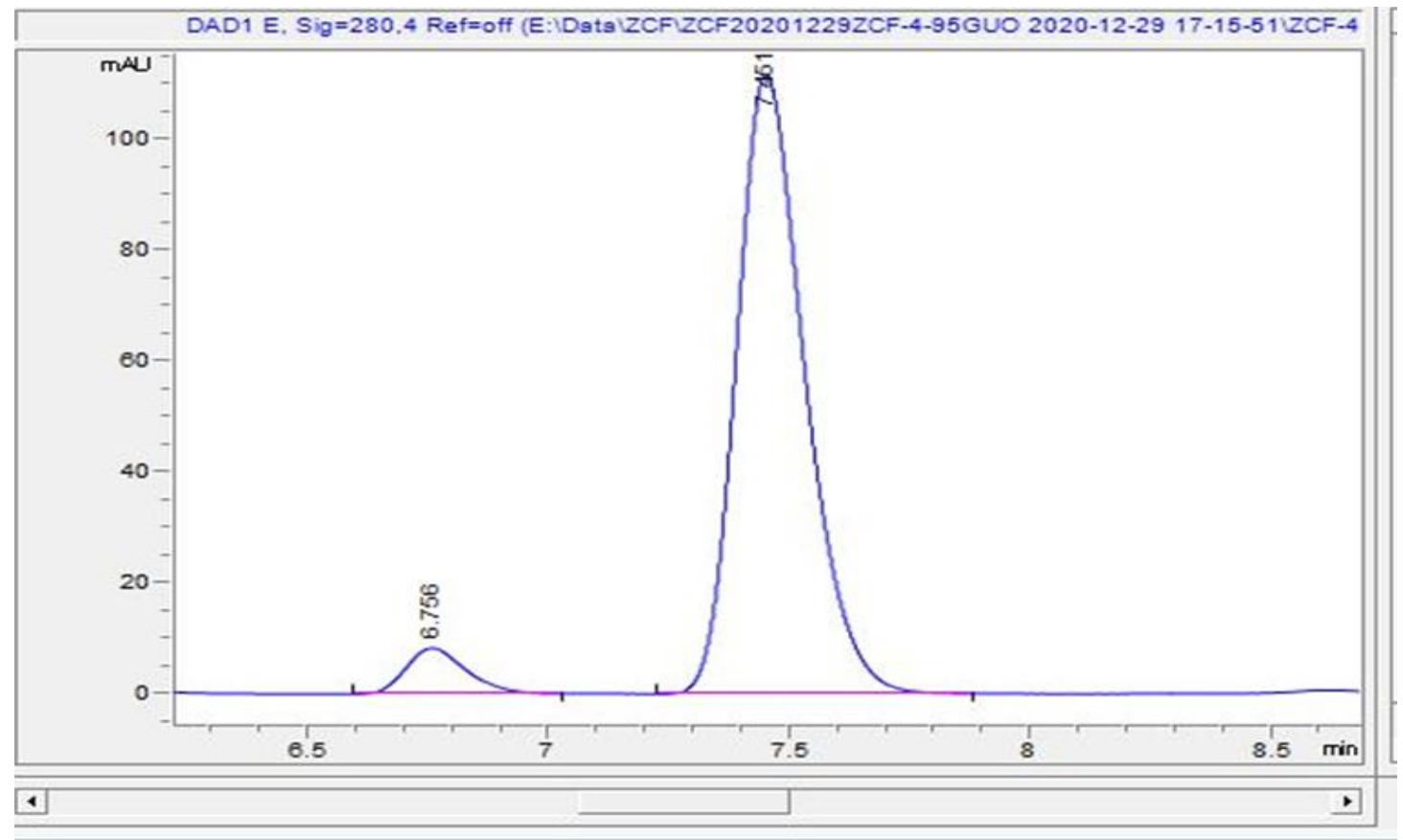

\begin{tabular}{|c|c|c|c|c|c|c|c|}
\hline \# & Time & Type & Area & Height & Width & Area\% & Symmetry \\
\hline 1 & 6.756 & $\mathrm{BB}$ & 71.4 & 8.2 & 0.1215 & 6.116 & 0.736 \\
\hline 2 & 7.451 & $B B$ & 1096 & 111.6 & 0.1506 & 93.884 & 0.725 \\
\hline
\end{tabular}


(R)-2-methyl-2-phenyl-1,2,3,4-tetrahydroquinoline $((R)$-1o)<smiles>C[C@]1(c2ccccc2)CCc2ccccc2N1</smiles>
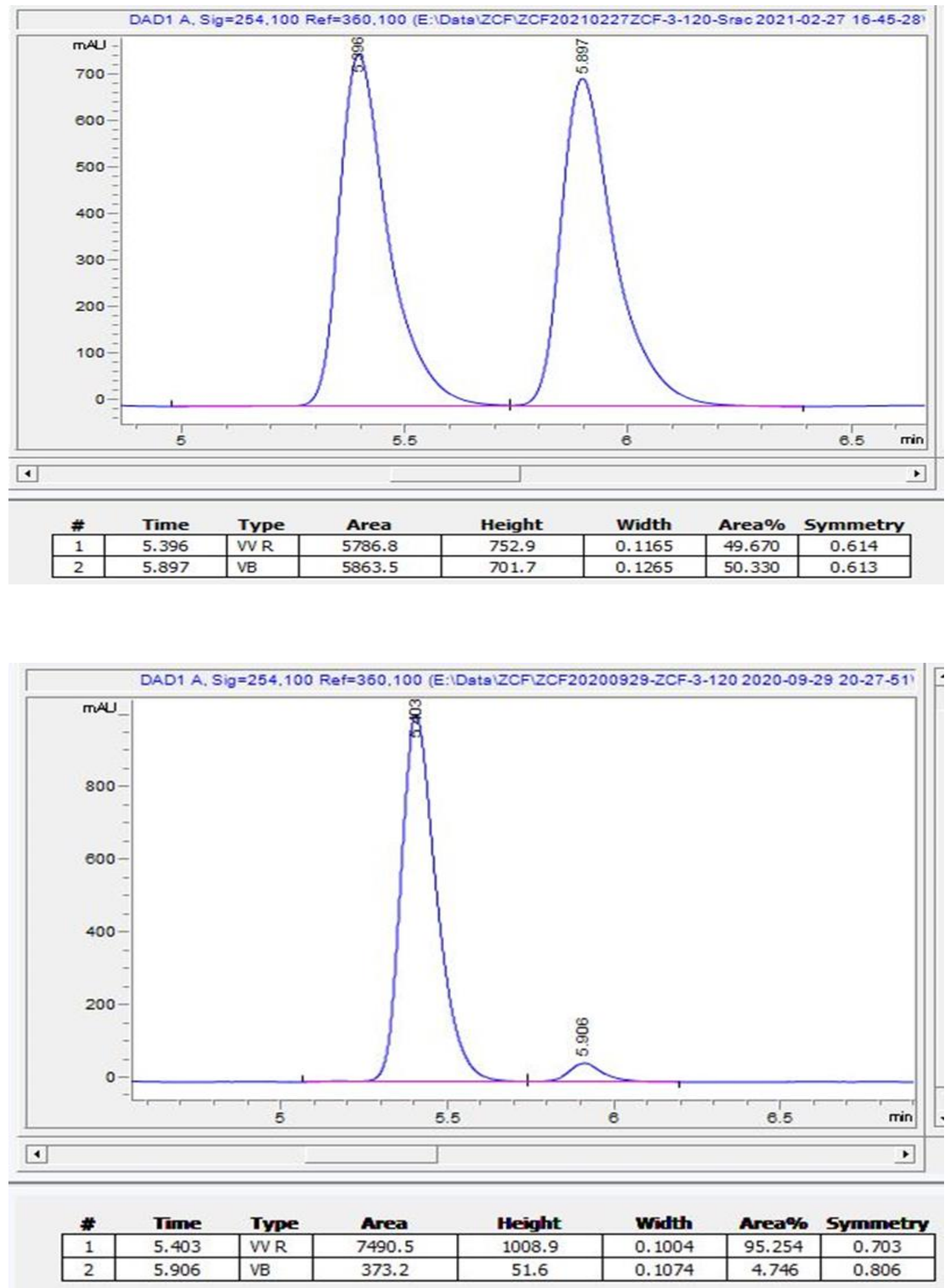
(S)-6-bromo-2-methyl-2-phenyl-1,2,3,4-tetrahydroquinoline ((S)-2o)<smiles>C[C@]1(c2ccccc2)CCc2cc(Br)ccc2N1</smiles>
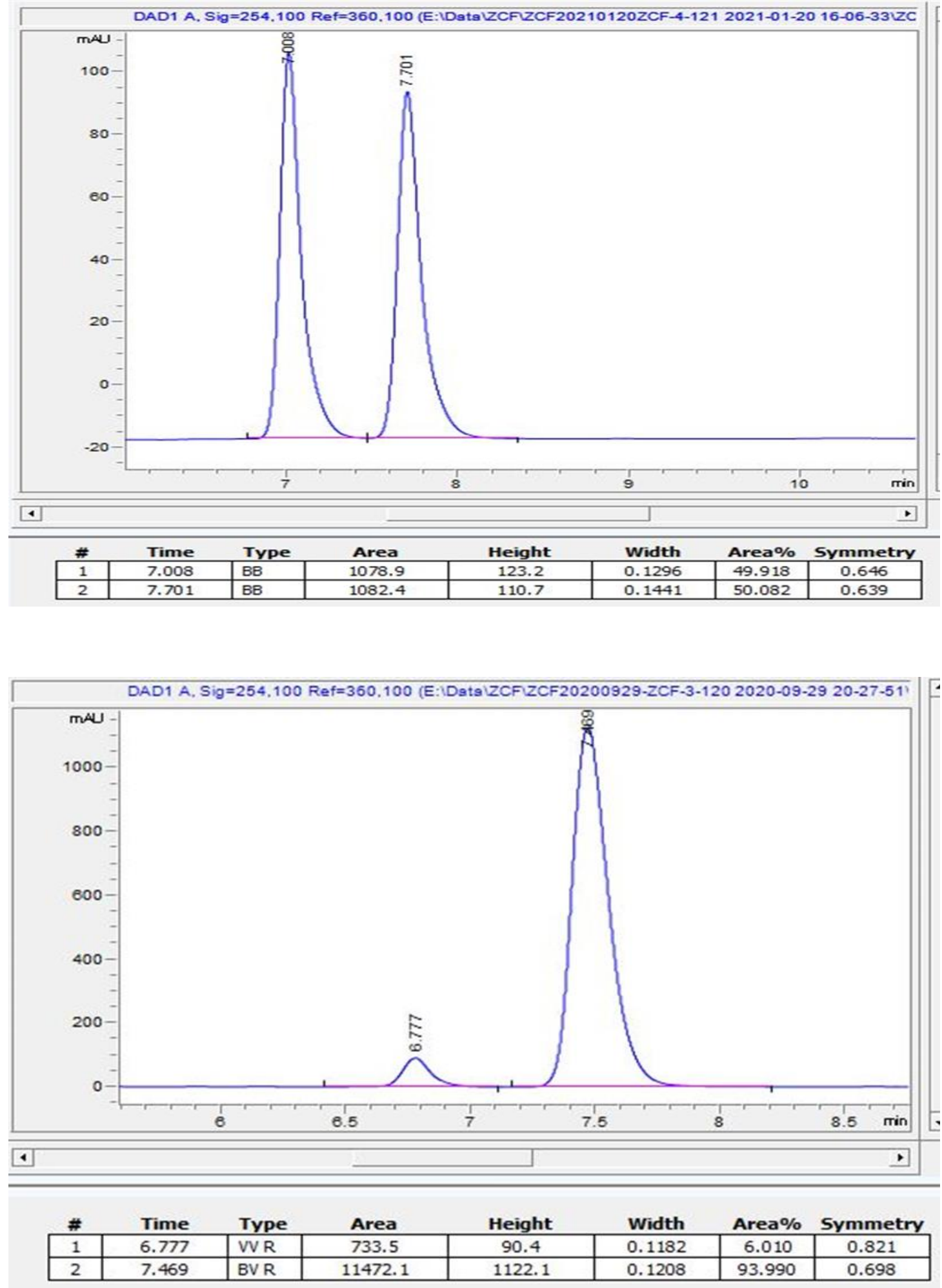
(S)-6-iodo-2-methyl-2-pheyl-1,2-dihydroquinoline ((S)-3a)<smiles>C[C@]1(c2ccccc2)C=Cc2cc(I)ccc2N1</smiles>
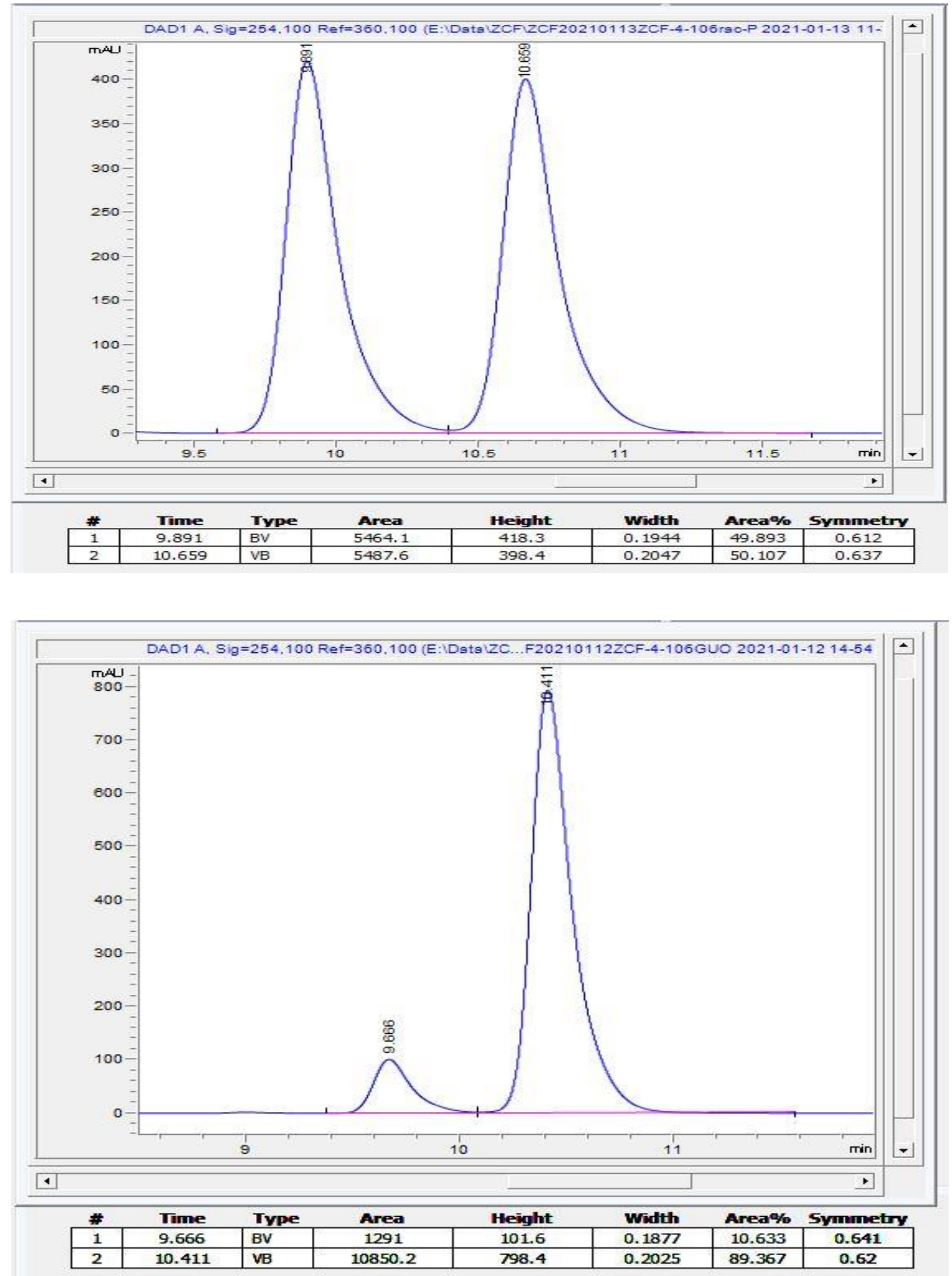
(S)-2-methyl-2-phenyl-6-(4,4,5,5-tetramethyl-1,3,2-dioxaborolan-2-yl)-1,2-dihydroquinoline $((S)-\mathbf{4 a})$
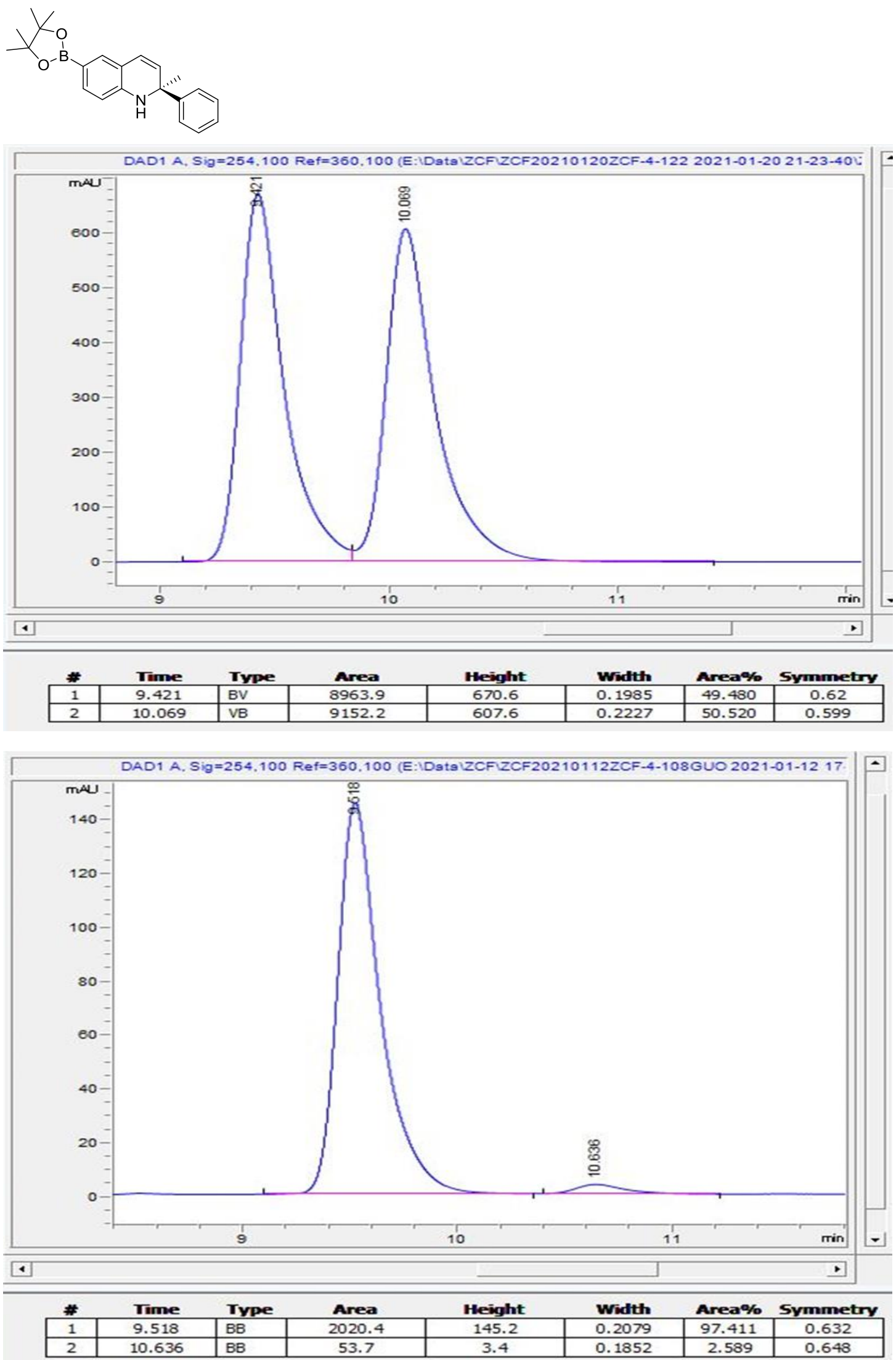
$(R)$-2,3-dimethyl-2-phenyl-1,2-dihydroquinoline $((R)$-1p)<smiles>CC1=Cc2ccccc2N[C@]1(C)c1ccccc1</smiles>
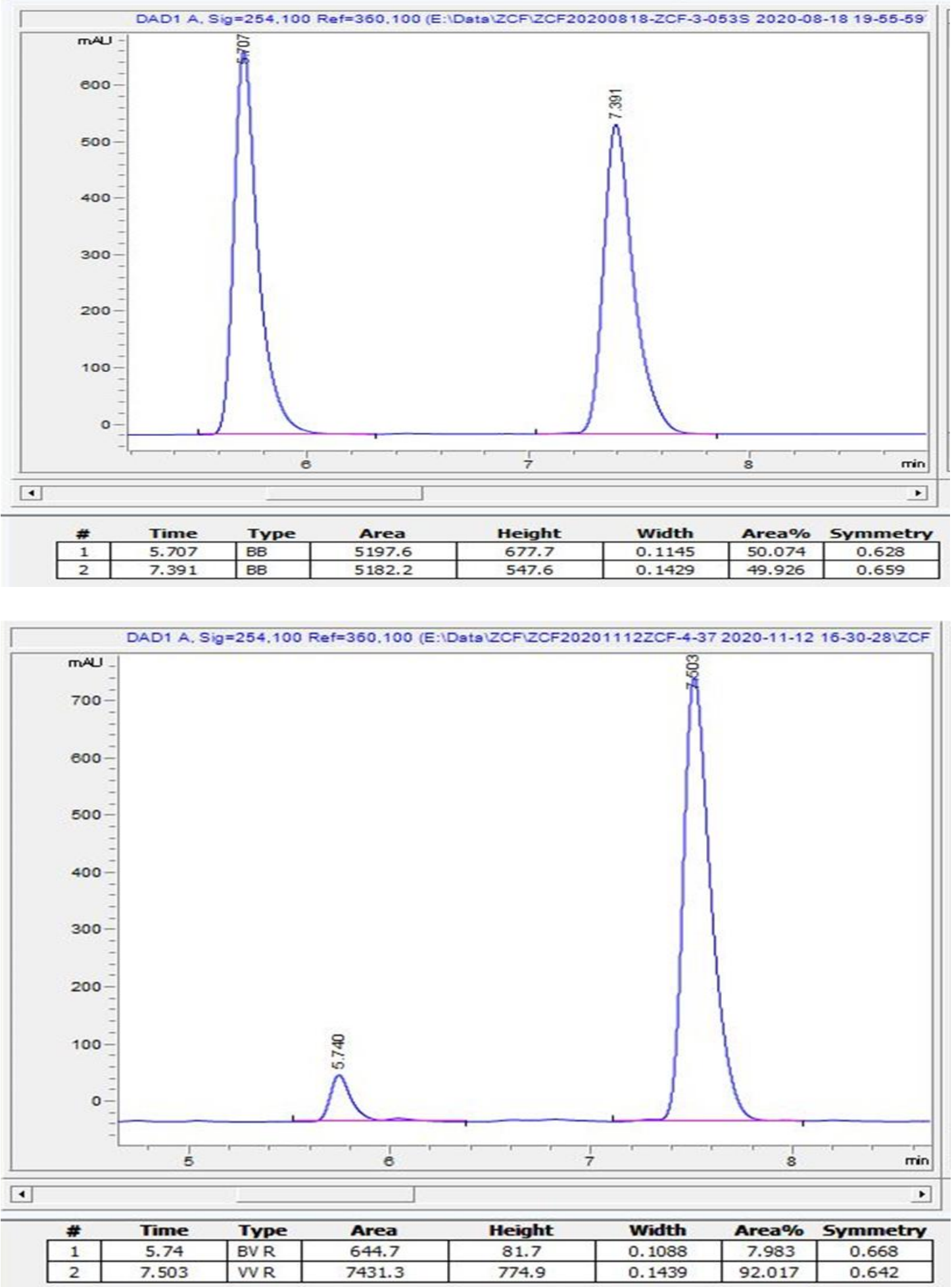
(S)-2,3-dimethyl-2-phenyl-6-(4,4,5,5-tetramethyl-1,3,2-dioxaborolan-2-yl)-1,2-dihydroquinol -ine $((S)-\mathbf{4 p})$<smiles>CC1=Cc2cc(B3OC(C)(C)C(C)(C)O3)ccc2NC1(c1ccccc1)c1ccccc1</smiles>
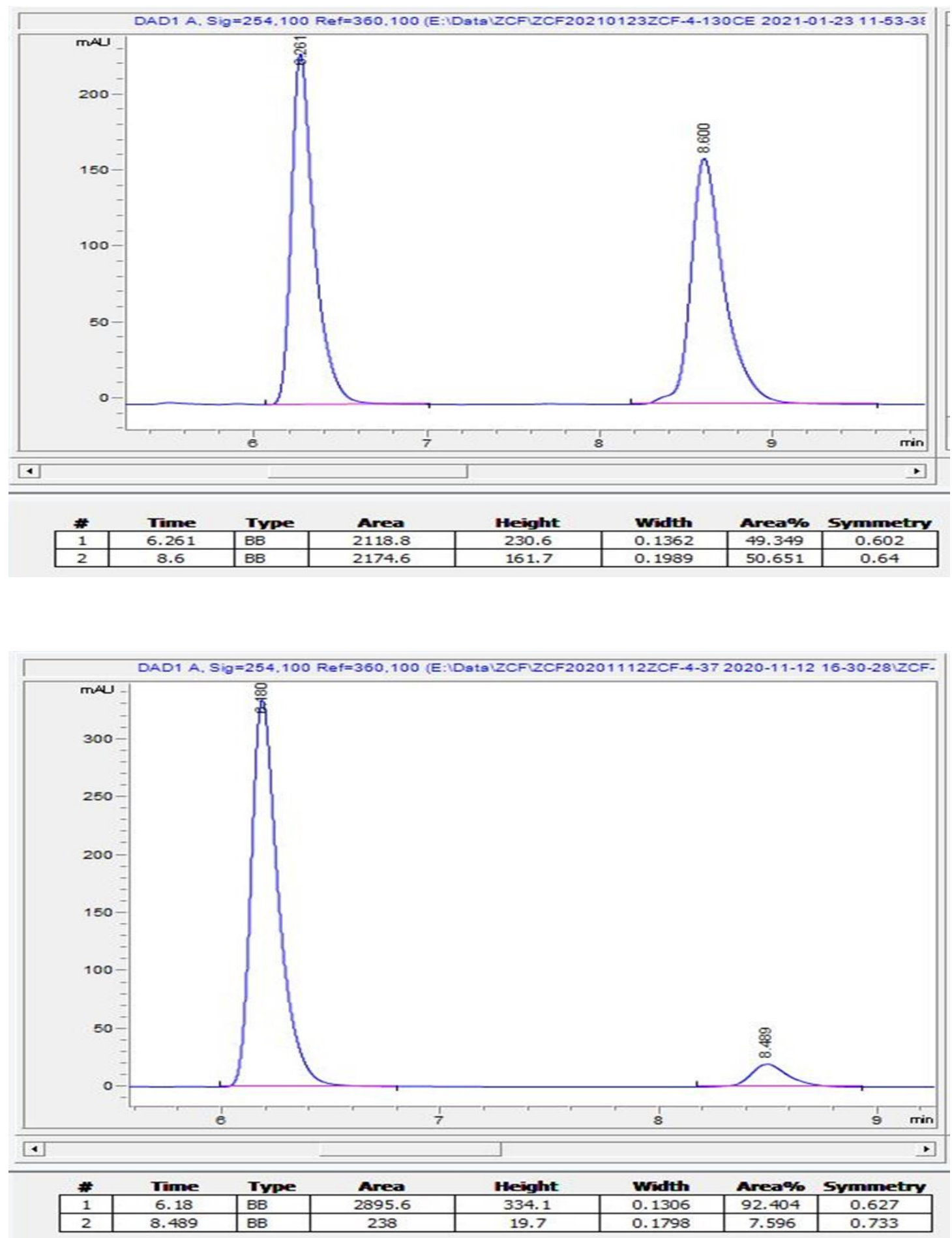
$(R)$-2-(3,5-dimethylphenyl)-2-methyl-1,2-dihydroquinoline ((R)-1q)<smiles>Cc1cc(C)cc(C2(C)C=Cc3ccccc3N2)c1</smiles>
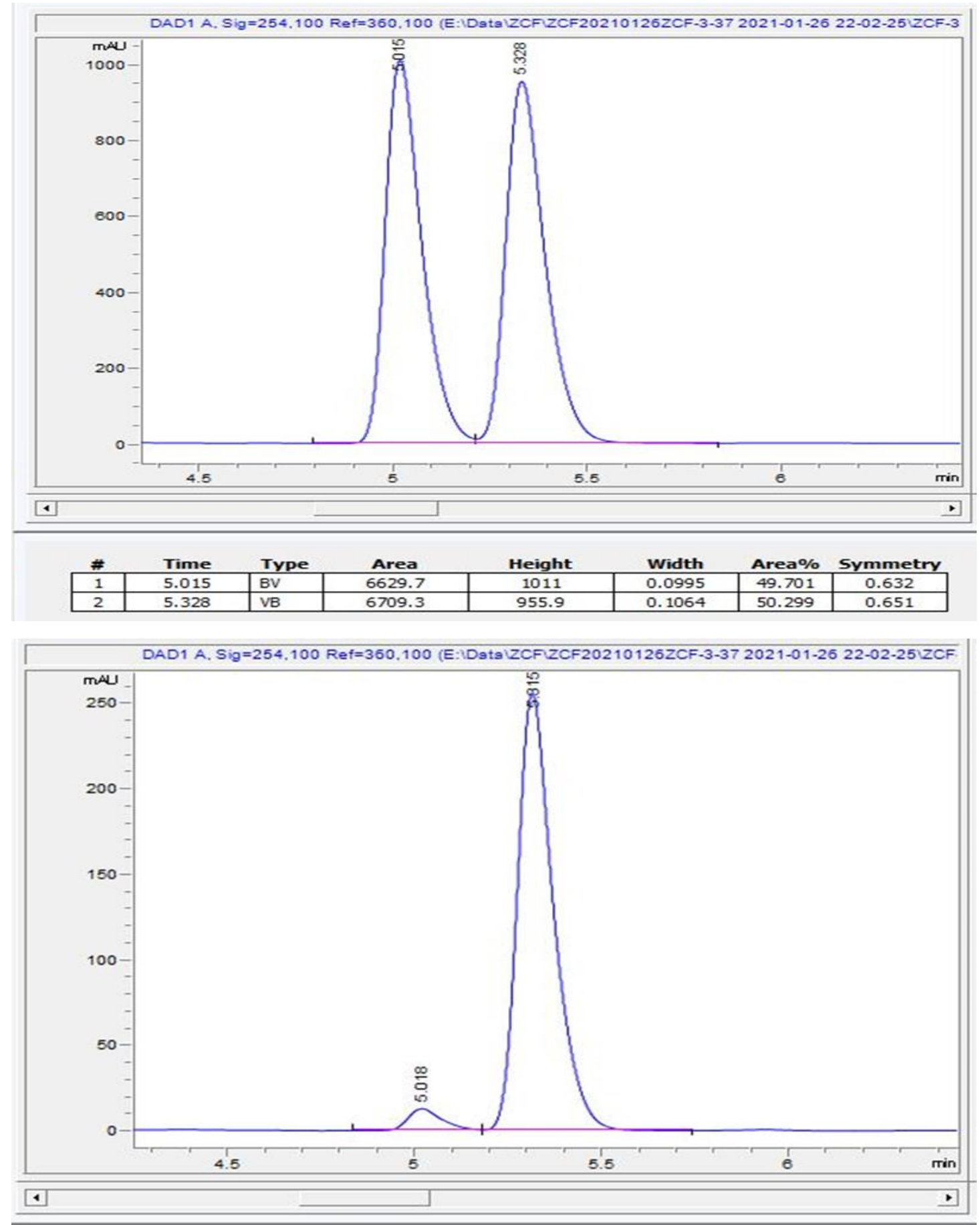

\begin{tabular}{|c|c|l|c|c|c|c|c|} 
\# & \multicolumn{1}{c}{ Time } & \multicolumn{1}{c}{ Type } & Area & Height & Width & \multicolumn{2}{c}{ Area\% $\%$ Symmetry } \\
\hline 1 & 5.018 & BV & 81.8 & 12.8 & 0.0949 & 4.532 & 0.652 \\
\hline 2 & 5.315 & VB & 1722.7 & 256.3 & 0.1009 & 95.468 & 0.655 \\
\hline
\end{tabular}


(S)-2-(3,5-dimethylphenyl)-2-methyl-6-(4,4,5,5-tetramethyl-1,3,2-dioxaborolan-2-yl)-1,2-dih ydroquinoline $((S)-\mathbf{4 q})$<smiles>Cc1cc(C)cc([C@]2(C)C=Cc3cc(B4OC(C)(C)C(C)(C)O4)ccc3N2)c1</smiles>
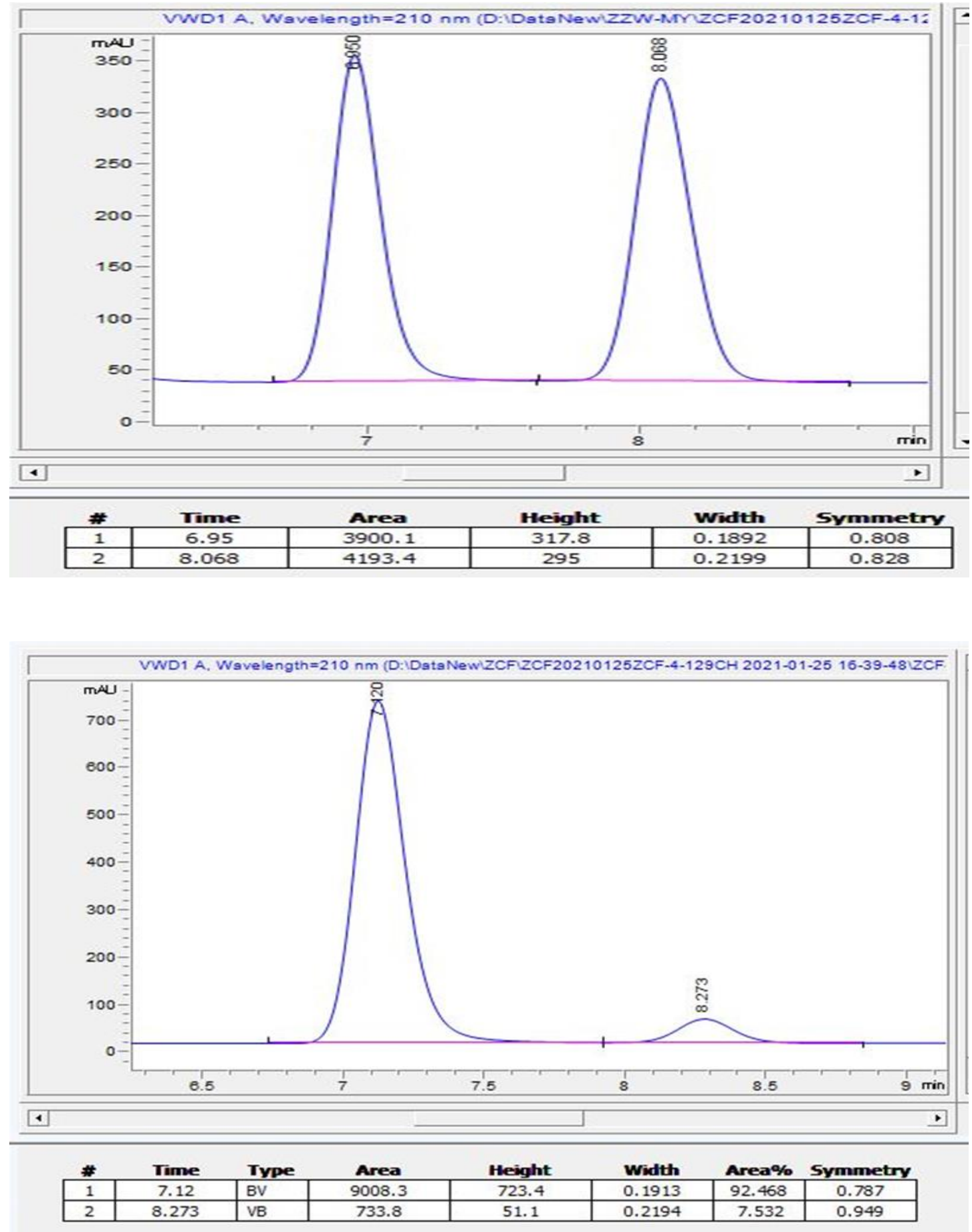
(2R,3R,4R)-6-bromo-3-iodo-4-methoxy-2-methyl-2-phenyl-1,2,3,4-tetrahydroquinoline $((S)-5 a)$<smiles>COC1c2cc(Br)ccc2NC(C)(c2ccccc2)C1I</smiles>
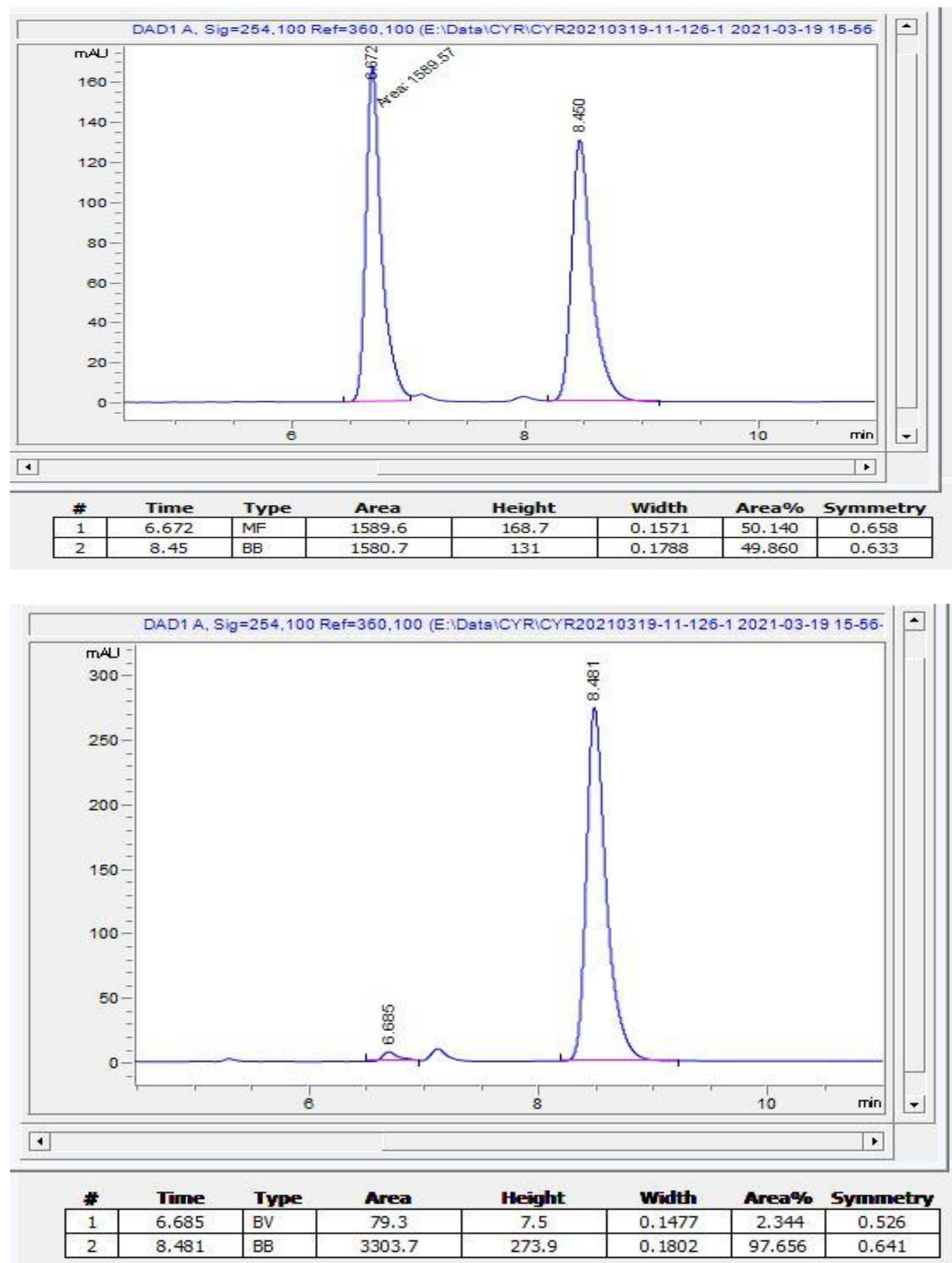
(S)-6-bromo-8-iodo-2-methyl-2-phenyl-1,2,3,4-tetrahydroquinoline $((S)-60)$<smiles>C[C@]1(c2ccccc2)CCc2cc(Br)cc(I)c2N1</smiles>
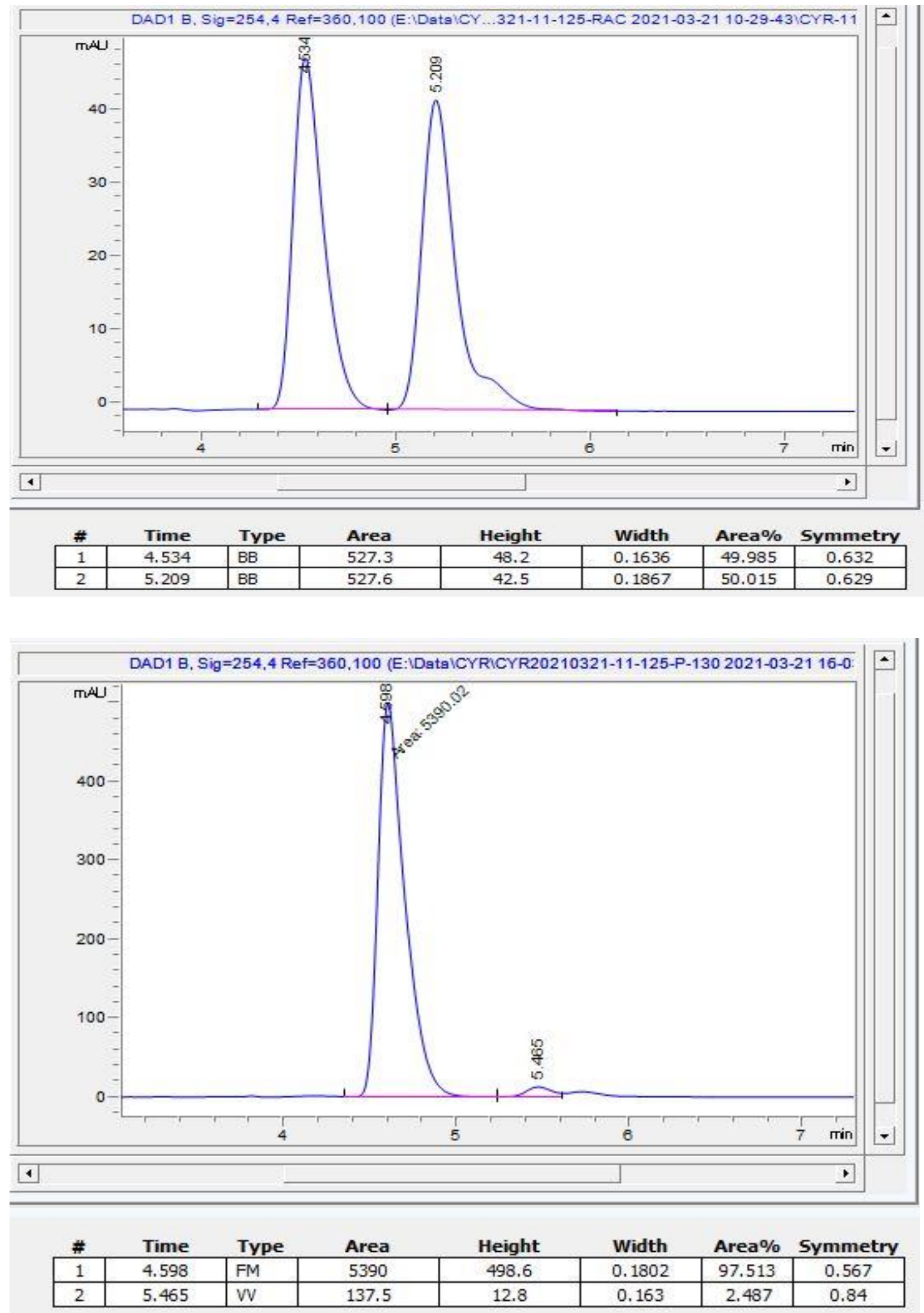


\section{NMR spectra}

$(R)$-2-methyl-2-phenyl-1,2-dihydroquinoline $((R)$-1a)
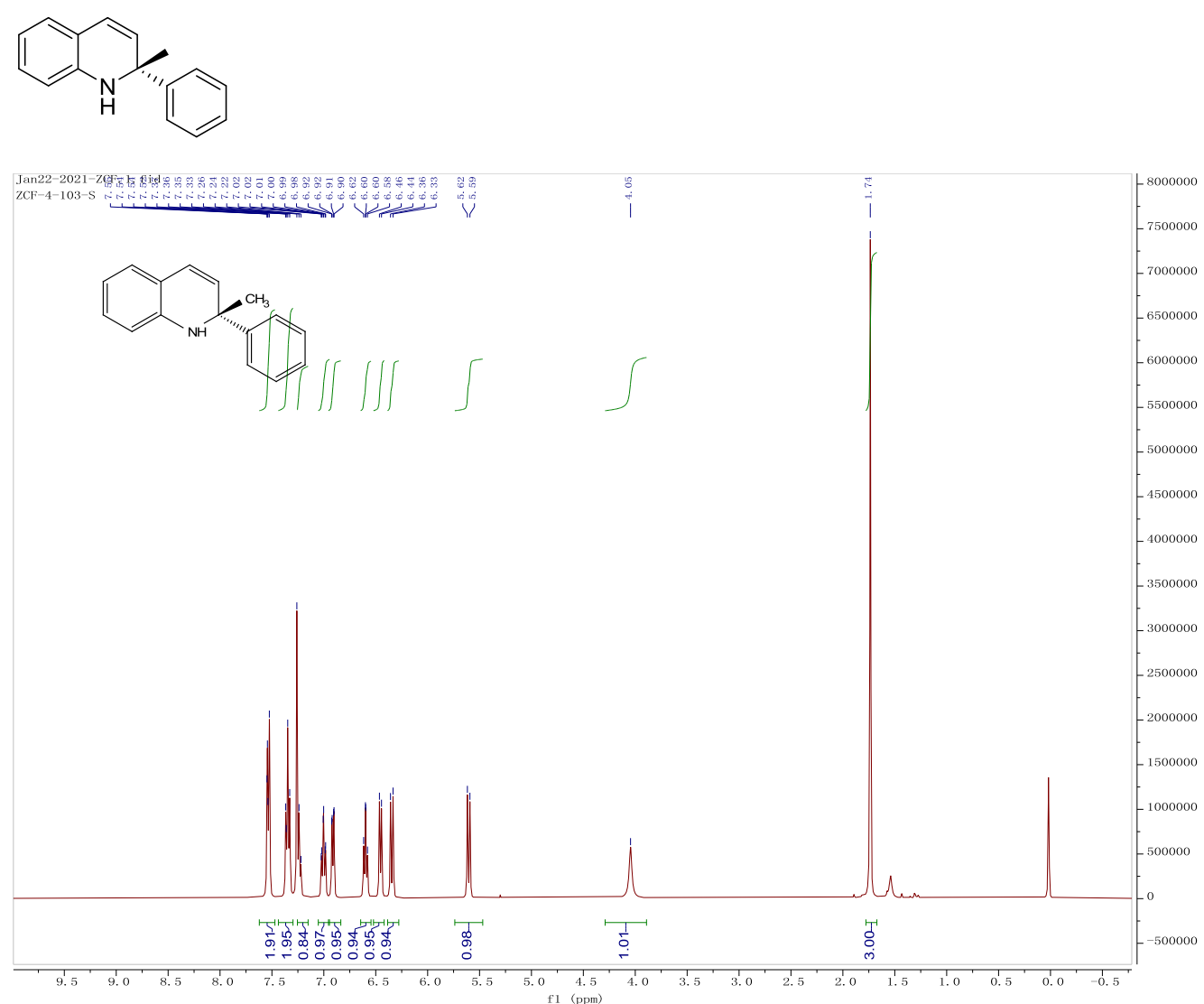

${ }^{1} \mathrm{H}$ NMR(400 MHz, Chloroform- $d$ )

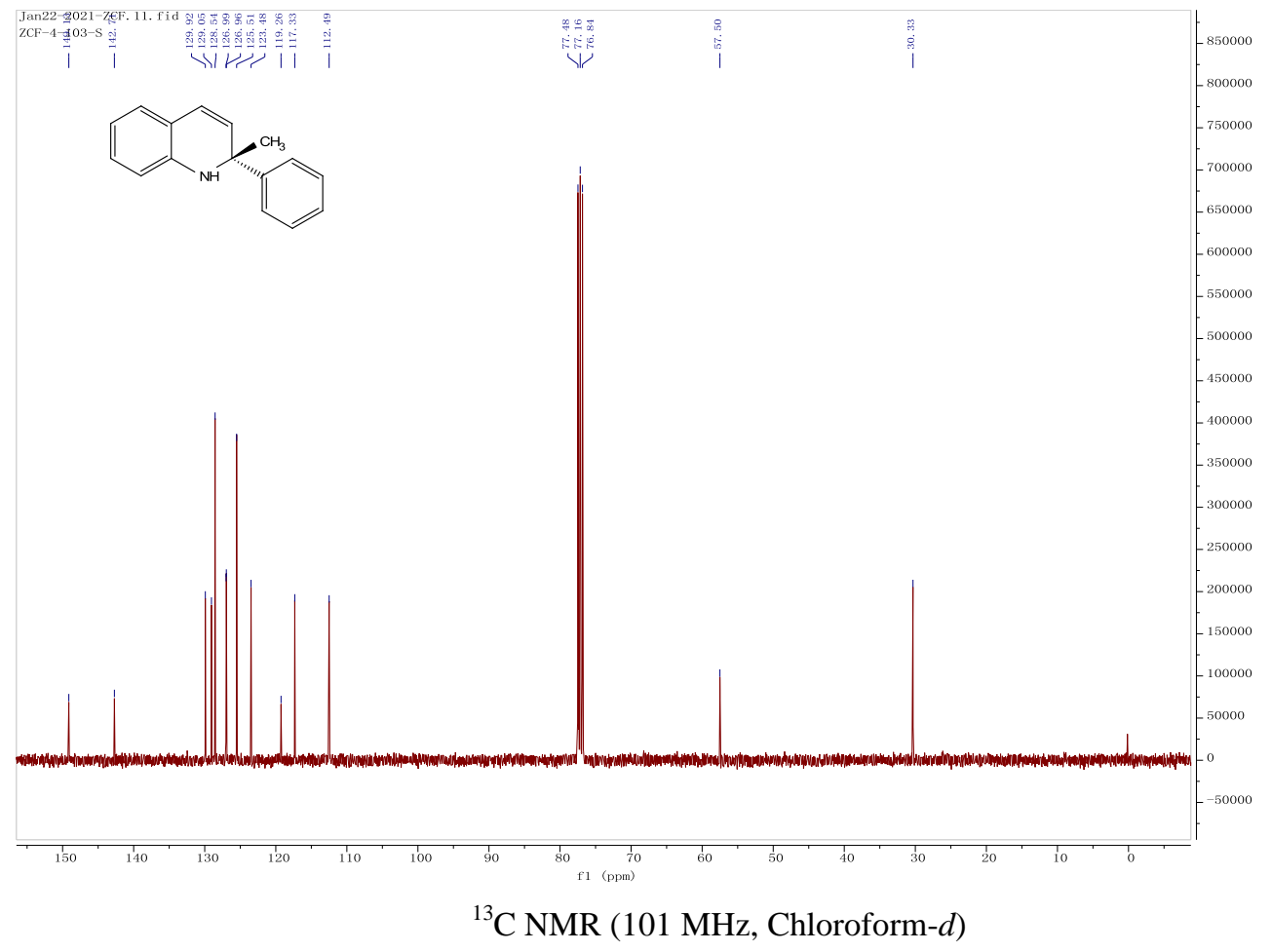


(S)-6-bromo-2-methyl-2-phenyl-1,2-dihydroquinoline ((S)-2a)

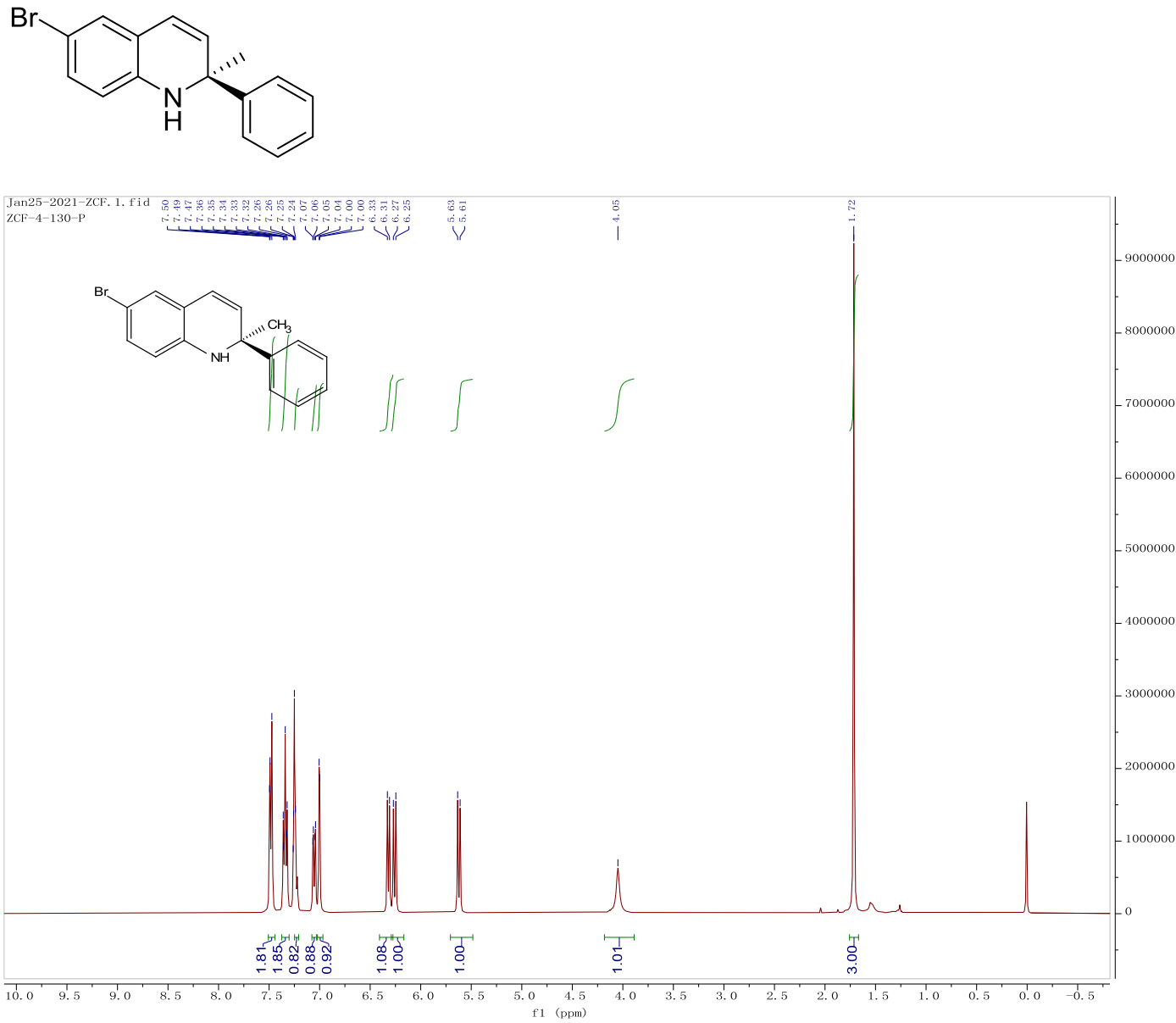

${ }^{1} \mathrm{H}$ NMR(400 MHz, Chloroform- $d$ )

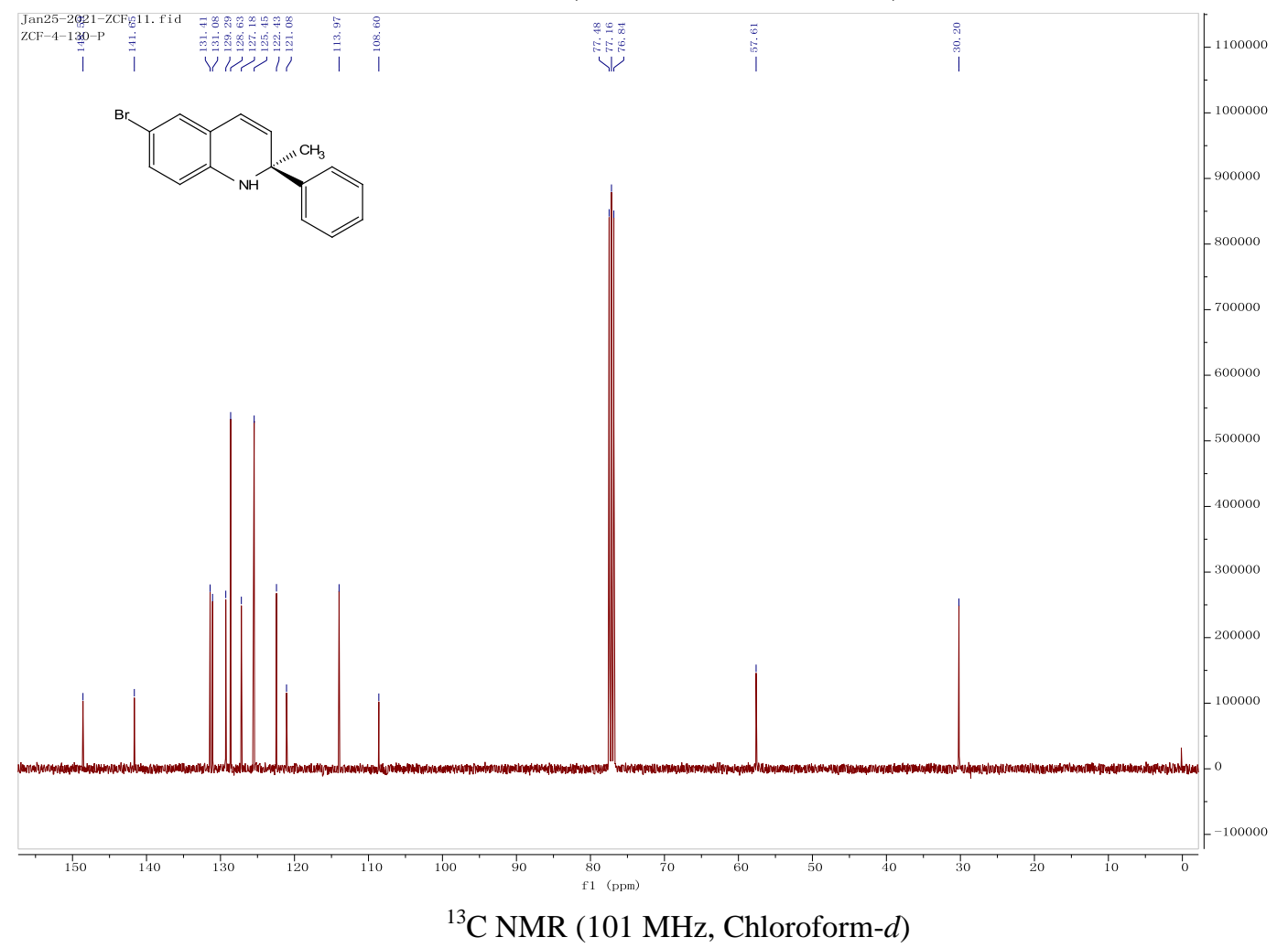


(R)-2-methyl-2-(p-tolyl)-1,2-dihydroquinoline $((R)$-1b)

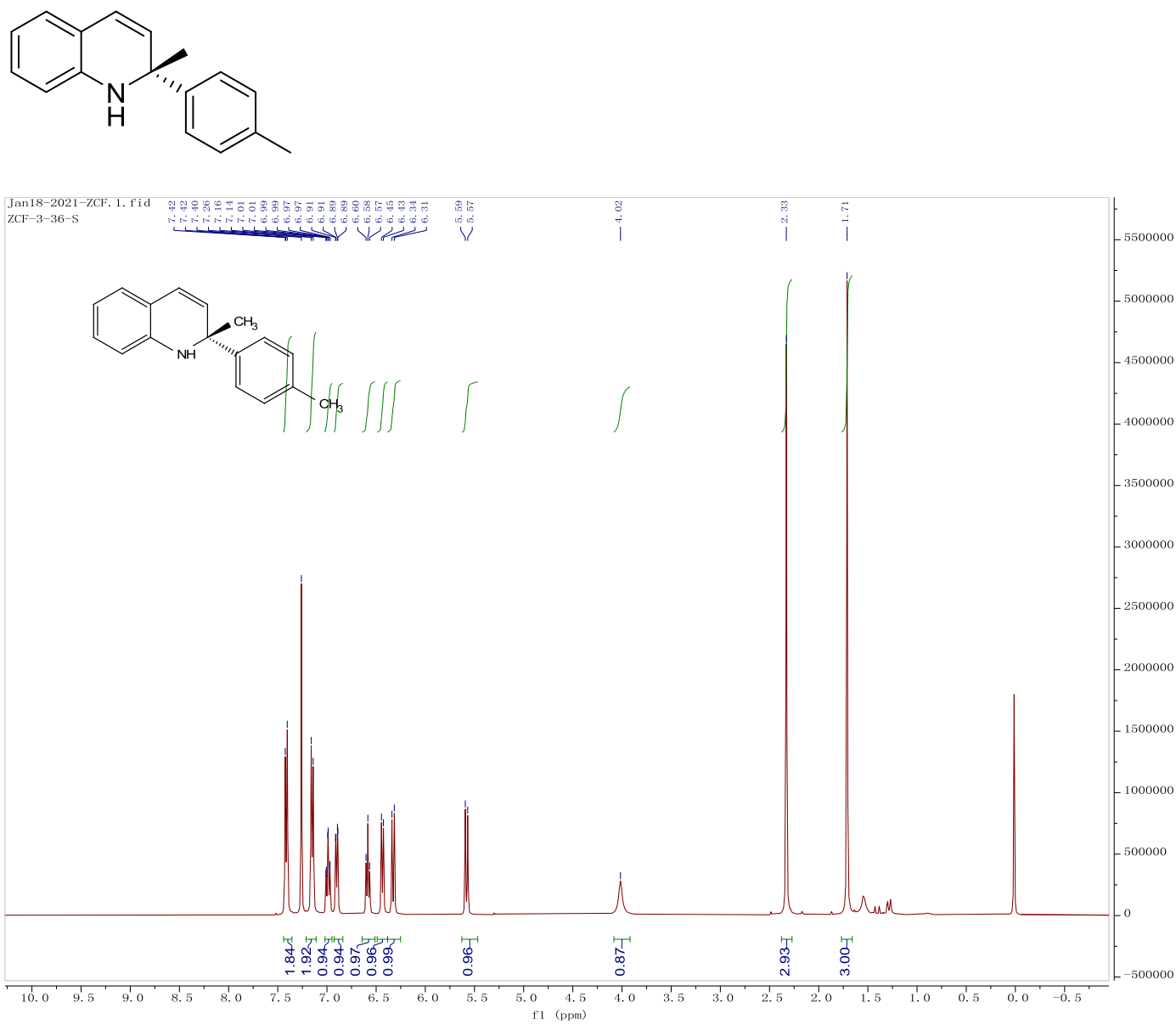

${ }^{1} \mathrm{H}$ NMR(400 MHz, Chloroform- $d$ )

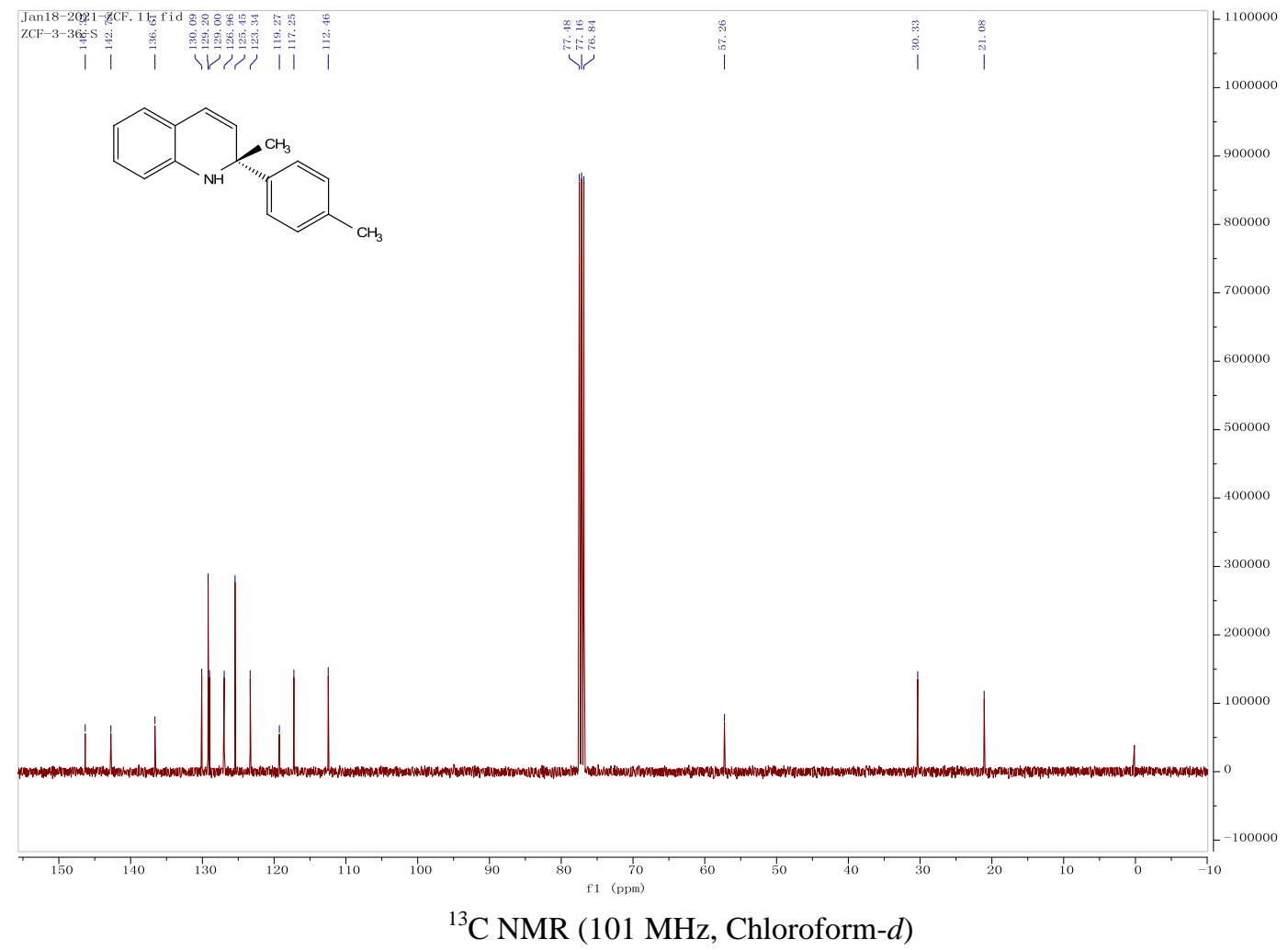


(S)-6-bromo-2-methyl-2-(p-tolyl)-1,2-dihydroquinoline ((S)-2b )

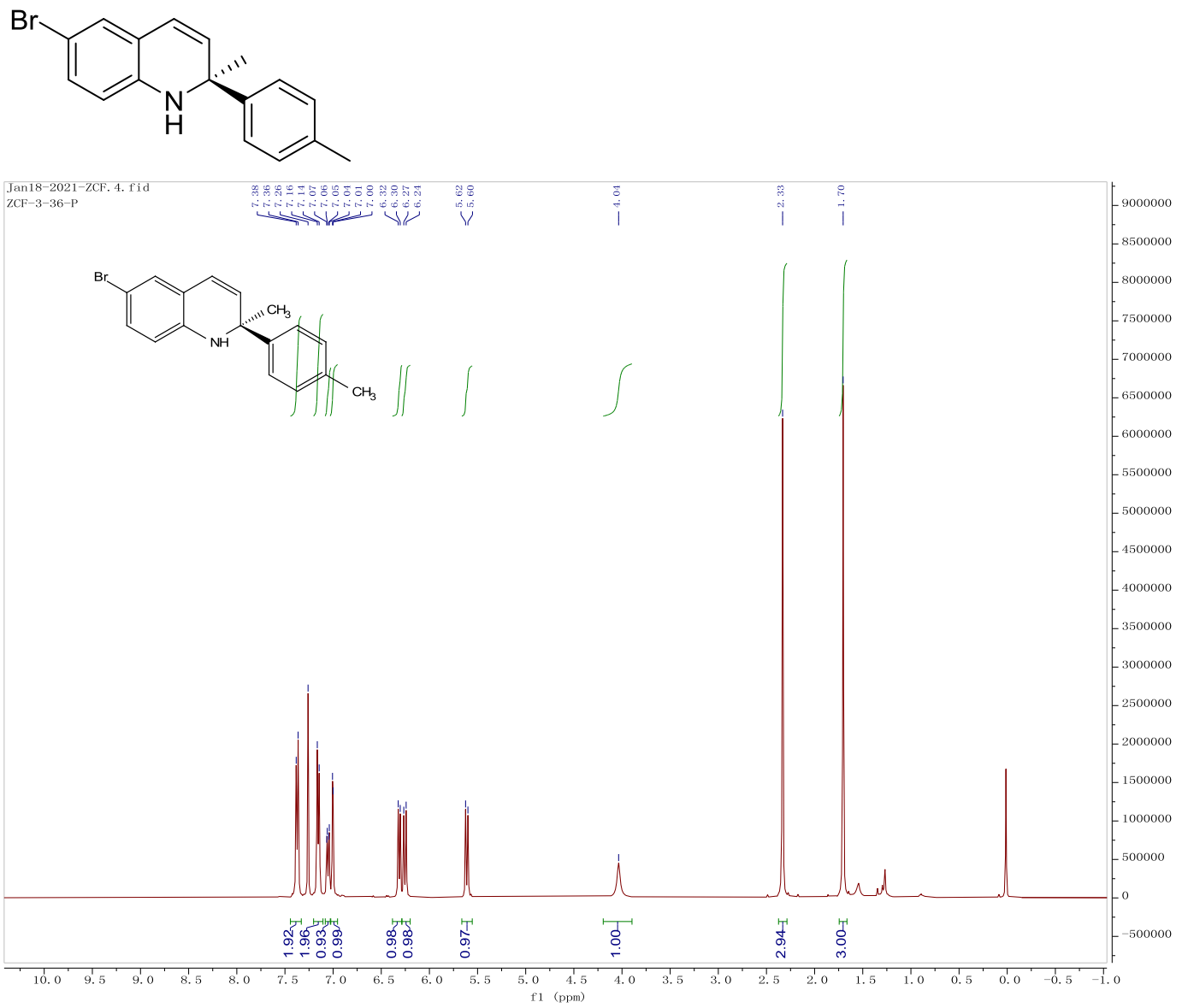

${ }^{1} \mathrm{H}$ NMR(400 MHz, Chloroform- $d$ )

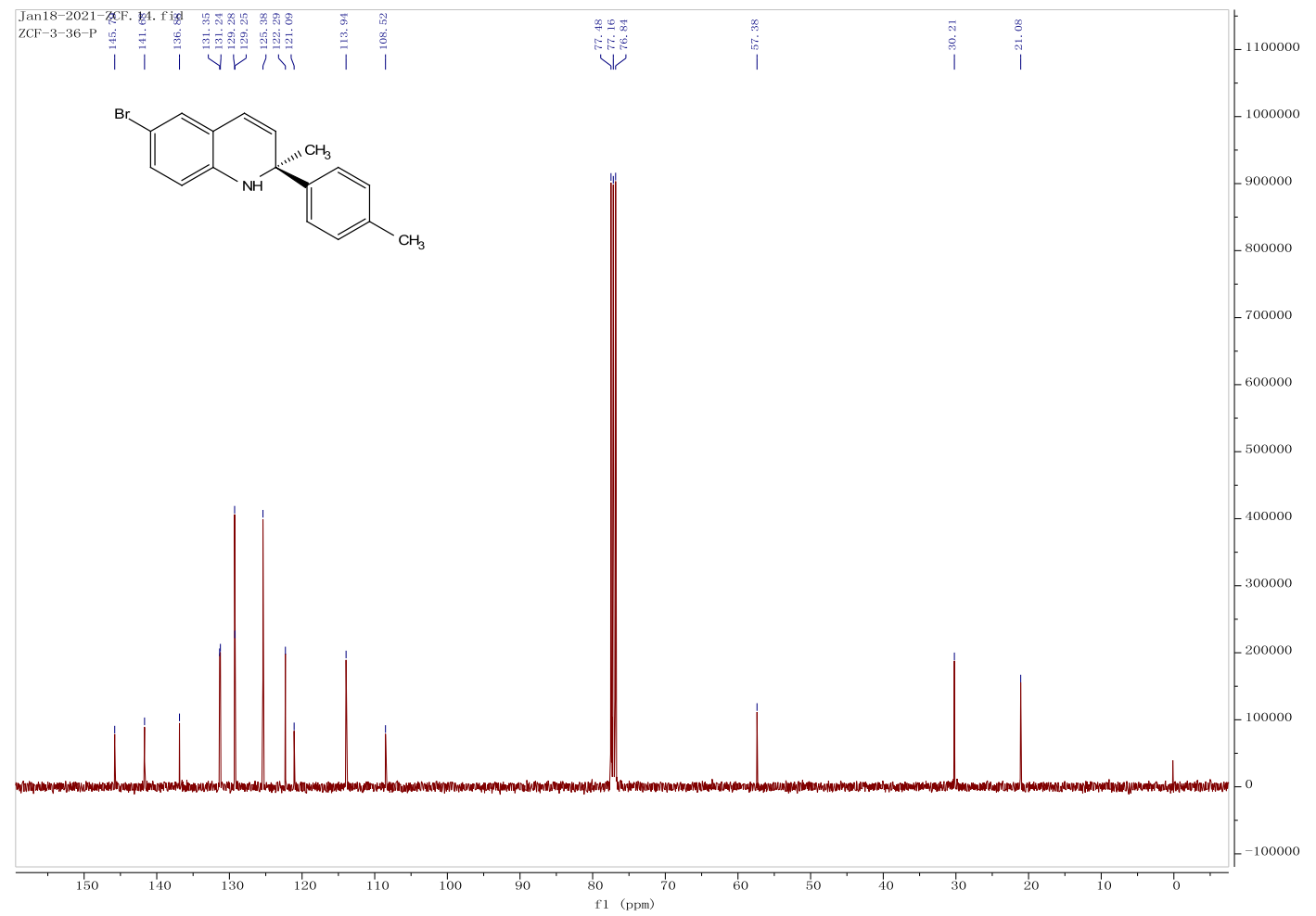

${ }^{13} \mathrm{C}$ NMR (101 MHz, Chloroform- $d$ ) 
(R)-2-(4-(tert-butyl)phenyl)-2-methyl-1,2-dihydroquinoline ((R)-1c)<smiles>CC(C)(C)c1ccc(C2(C)C=Cc3ccccc3N2)cc1</smiles>

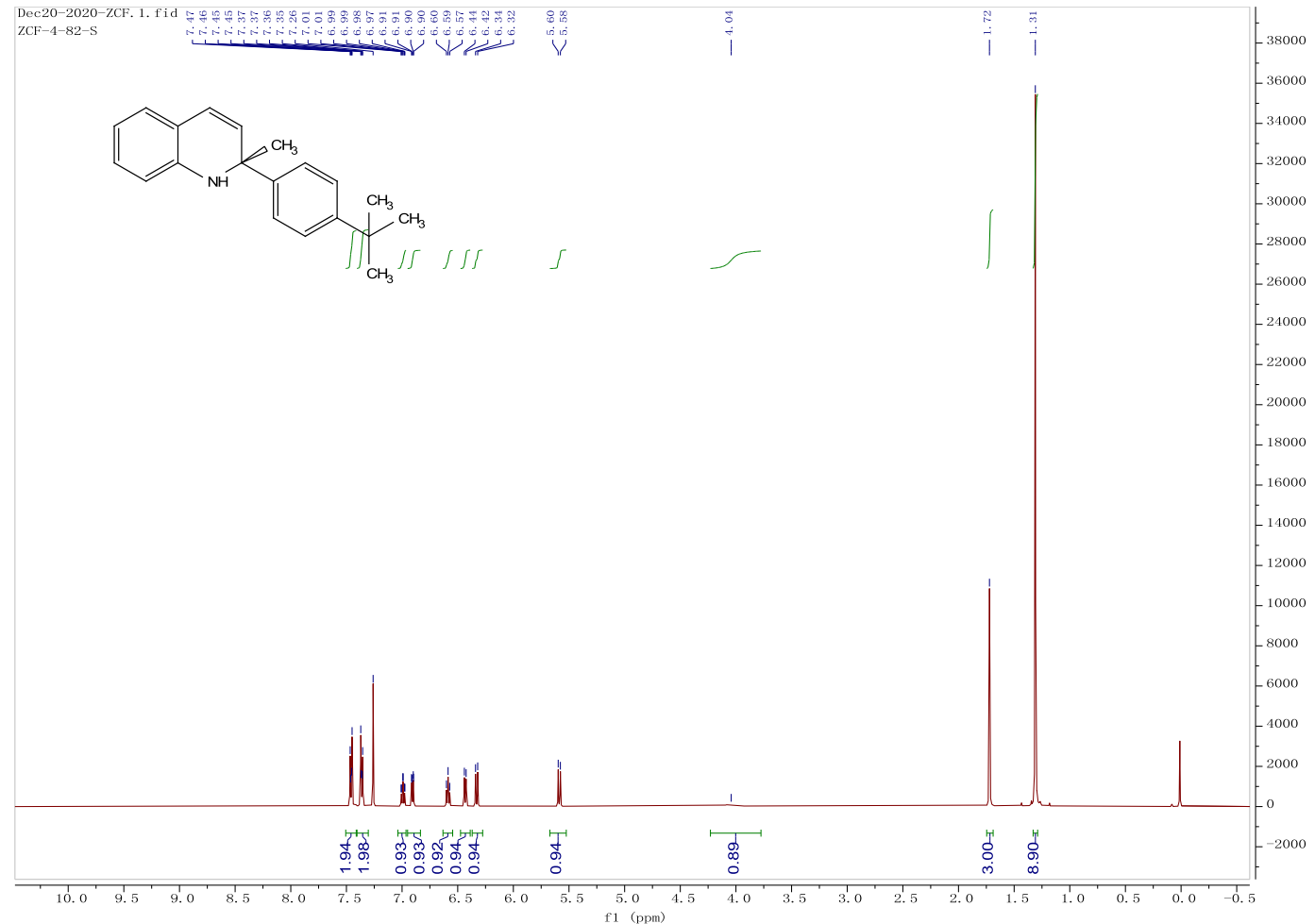

${ }^{1} \mathrm{H}$ NMR(500 MHz, Chloroform- $d$ )

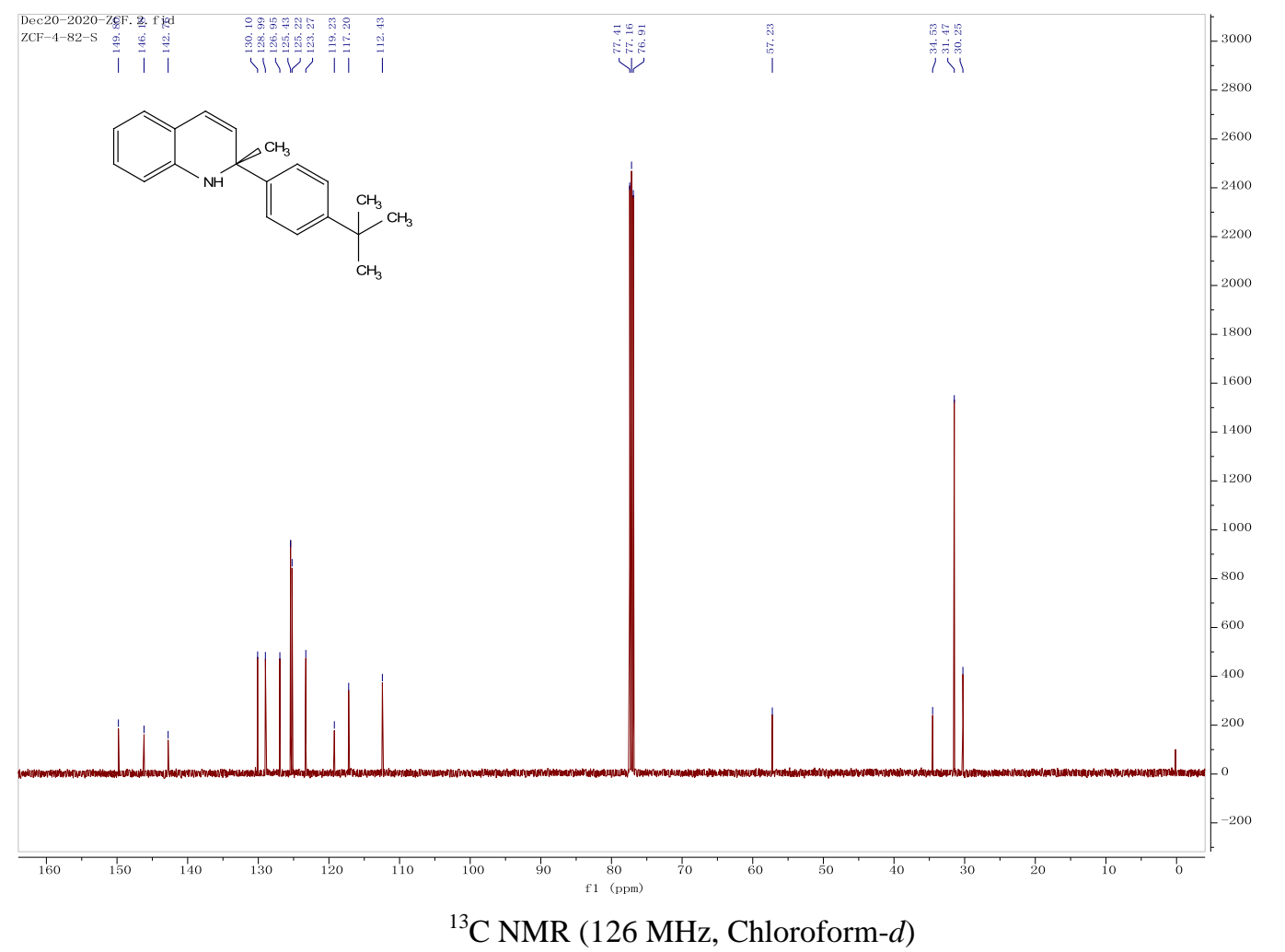


(S)-6-bromo-2-(4-(tert-butyl)phenyl)-2-methyl-1,2-dihydroquinoline ((S)-2c)<smiles>CC(C)(C)c1ccc([C@]2(C)C=Cc3cc(Br)ccc3N2)cc1</smiles>

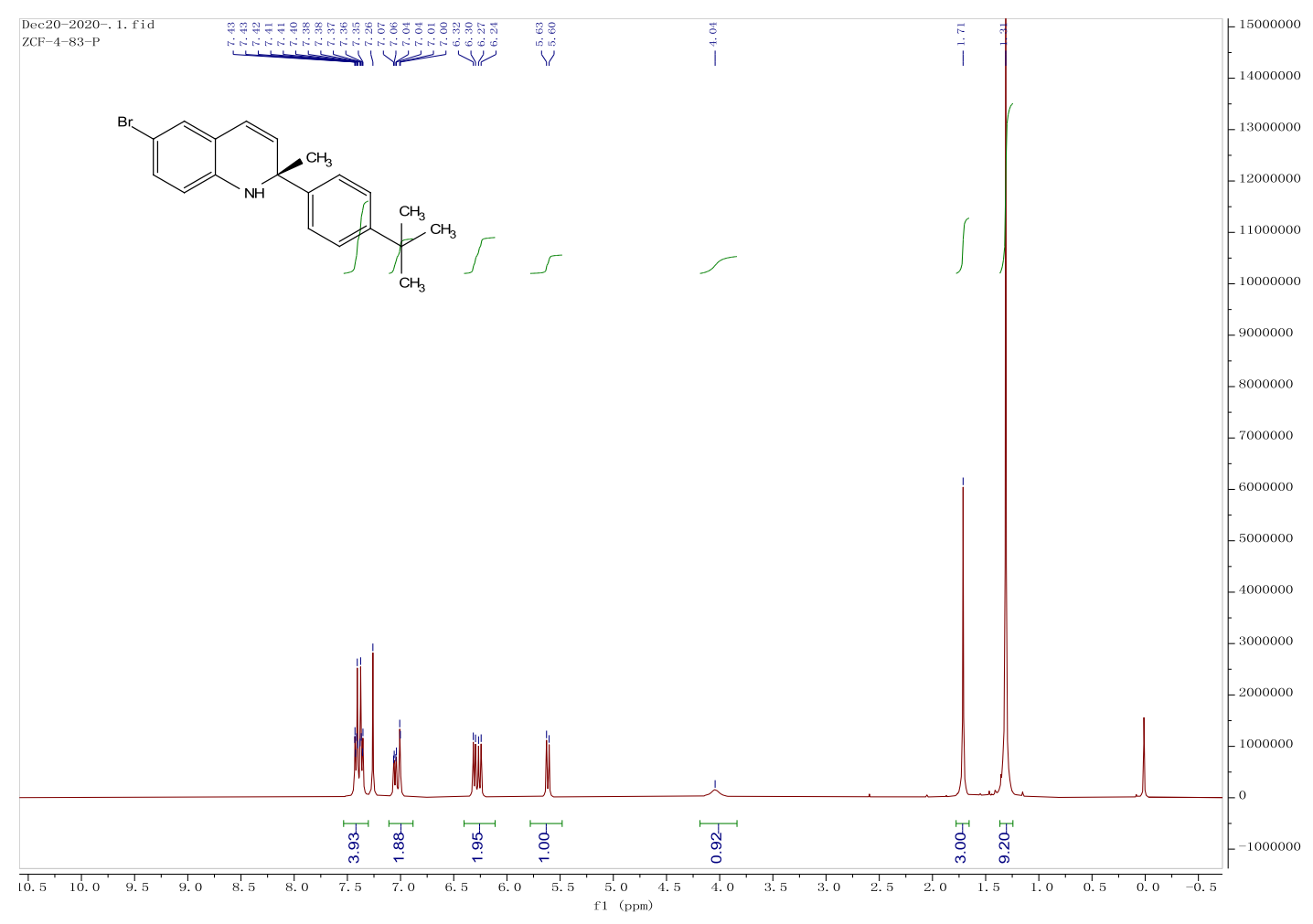

${ }^{1} \mathrm{H}$ NMR(400 MHz, Chloroform- $d$ )

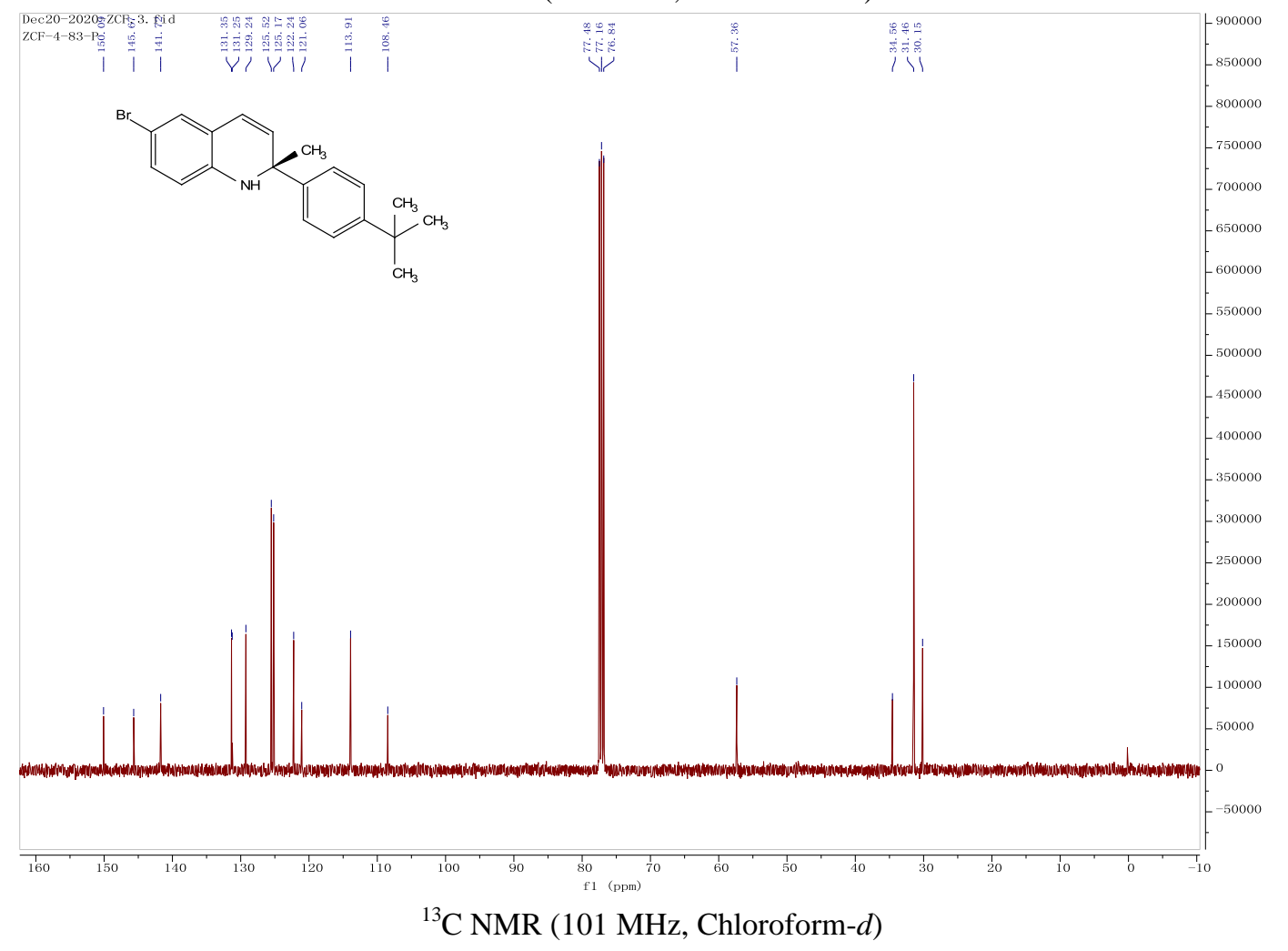


(R)-2-([1,1'-biphenyl]-4-yl)-2-methyl-1,2-dihydroquinoline ((R)-1d)<smiles>C[C@]1(c2ccc(-c3ccccc3)cc2)C=Cc2ccccc2N1</smiles>

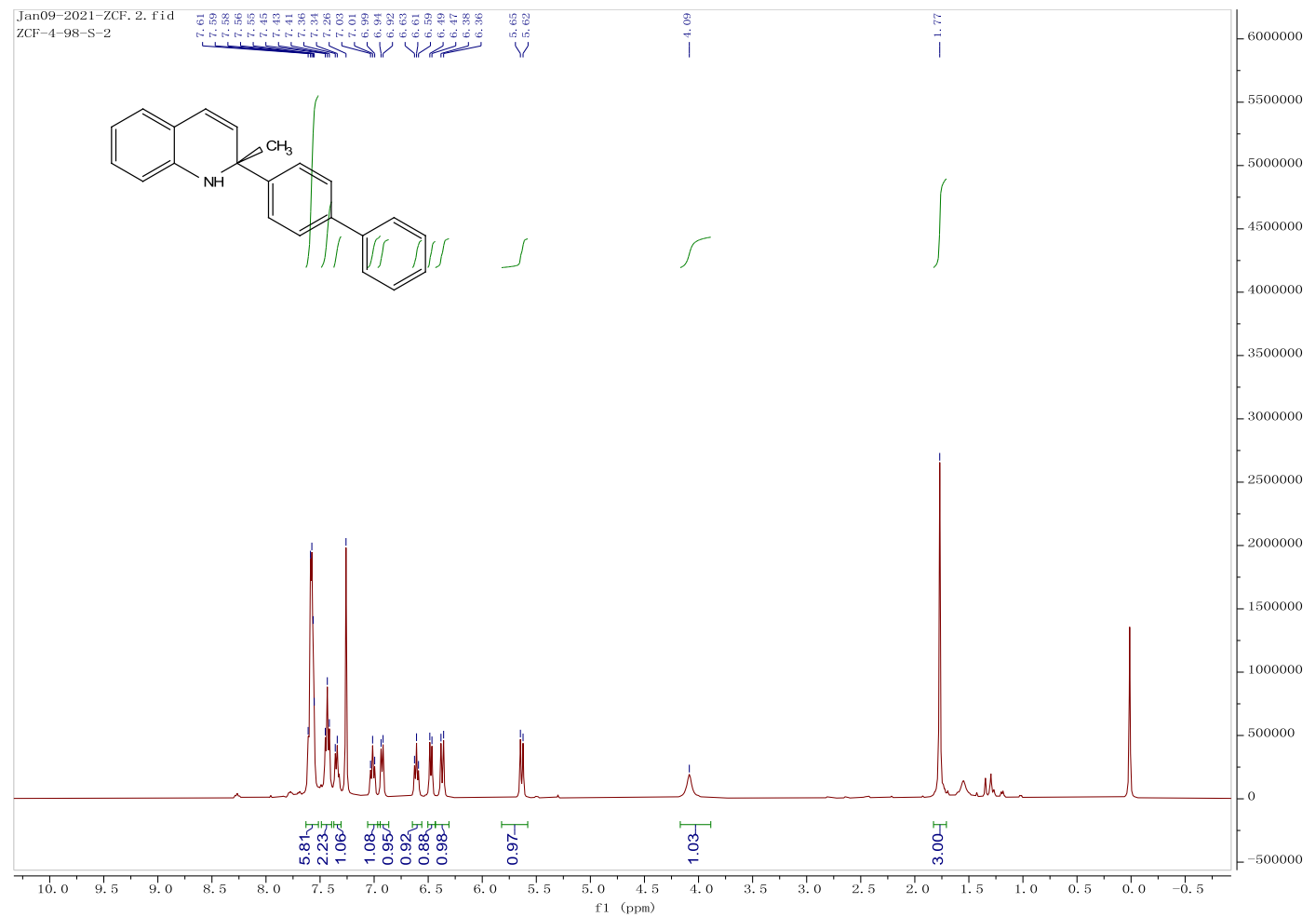

${ }^{1} \mathrm{H}$ NMR(400 MHz, Chloroform- $d$ )

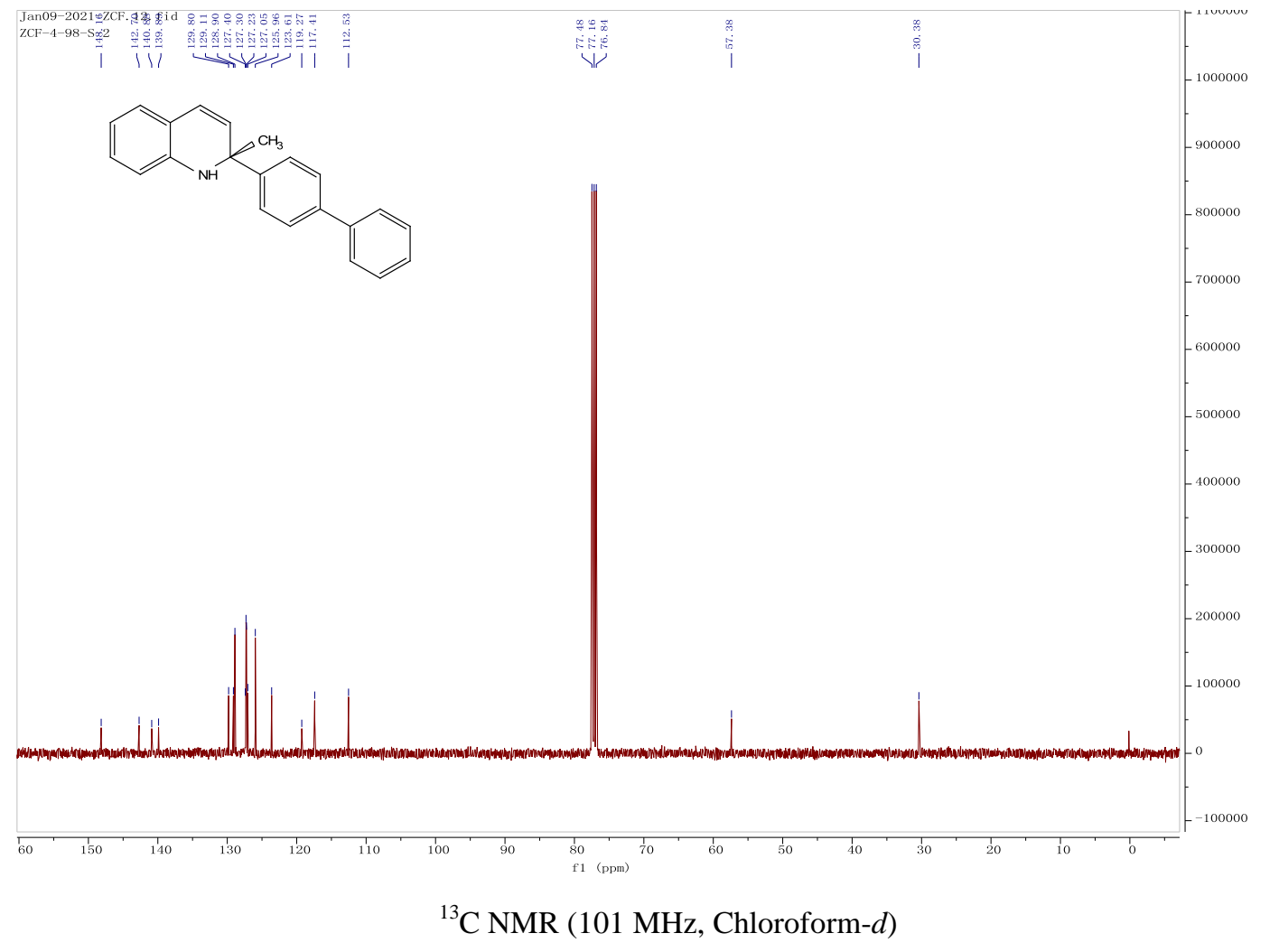


(S)-2-([1,1'-biphenyl]-4-yl)-6-bromo-2-methyl-1,2-dihydroquinoline ((S)-2d)<smiles>C[C@]1(c2ccc(-c3ccccc3)cc2)C=Cc2cc(Br)ccc2N1</smiles>

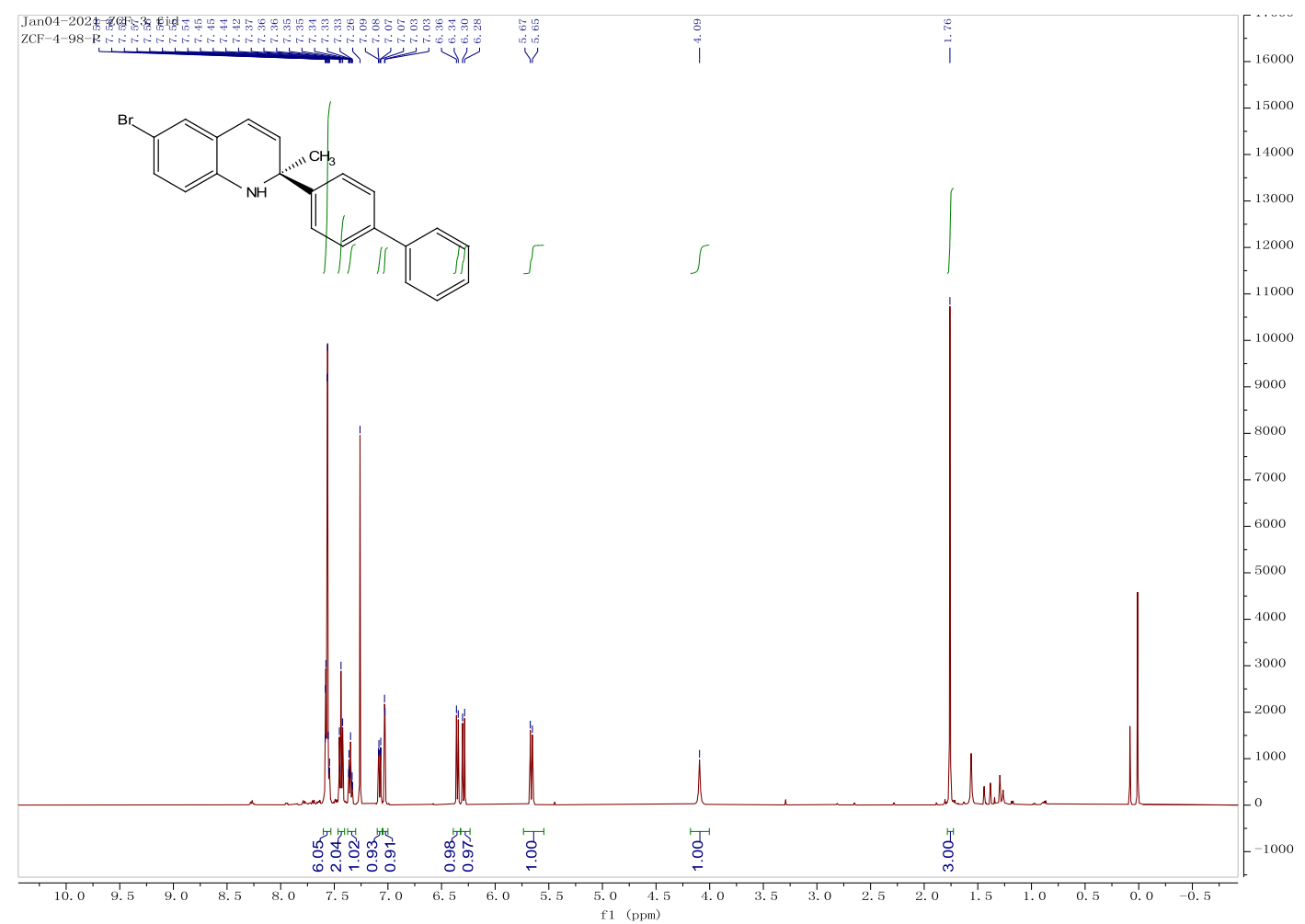

${ }^{1} \mathrm{H}$ NMR $(500 \mathrm{MHz}$, Chloroform- $d)$

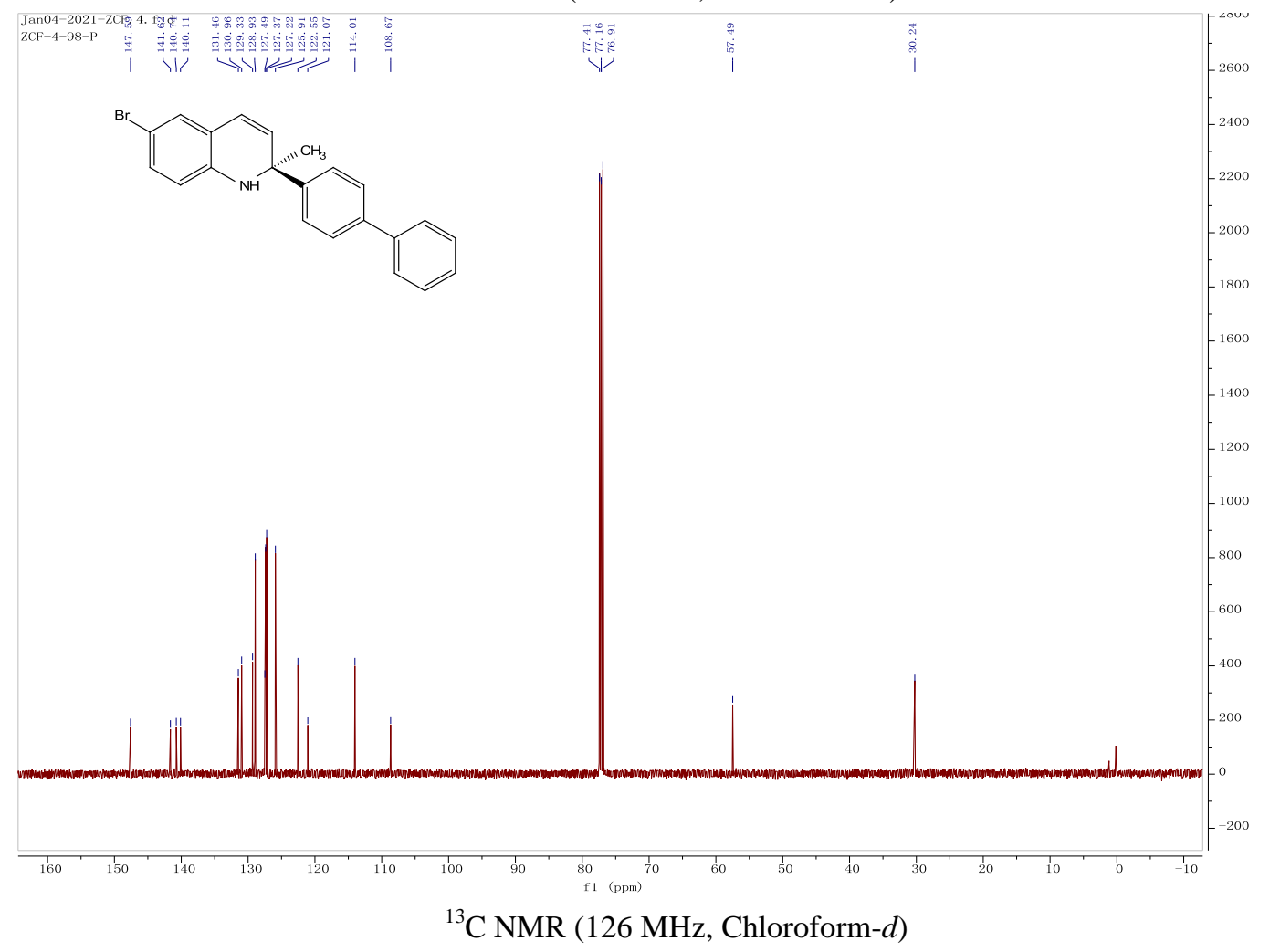


(R)-2-(4-methoxyphenyl)-2-methyl-1,2-dihydroquinoline ((R)-1e)<smiles>COc1ccc([C@]2(C)C=Cc3ccccc3N2)cc1</smiles>

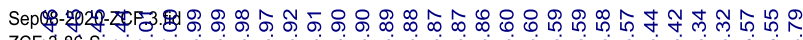
ZCFA3-8G-S
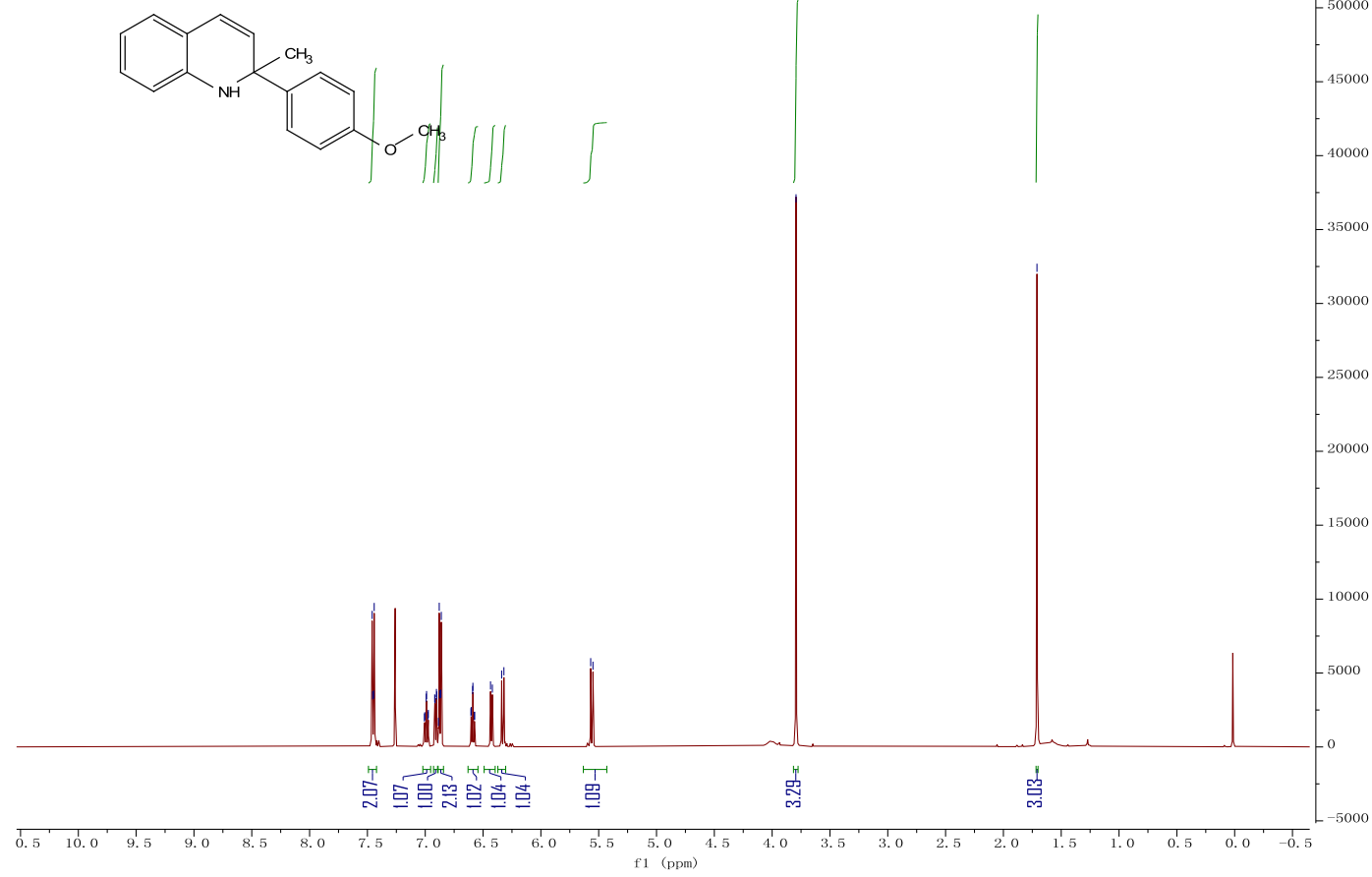

${ }^{1} \mathrm{H}$ NMR(500 MHz, Chloroform- $d$ )

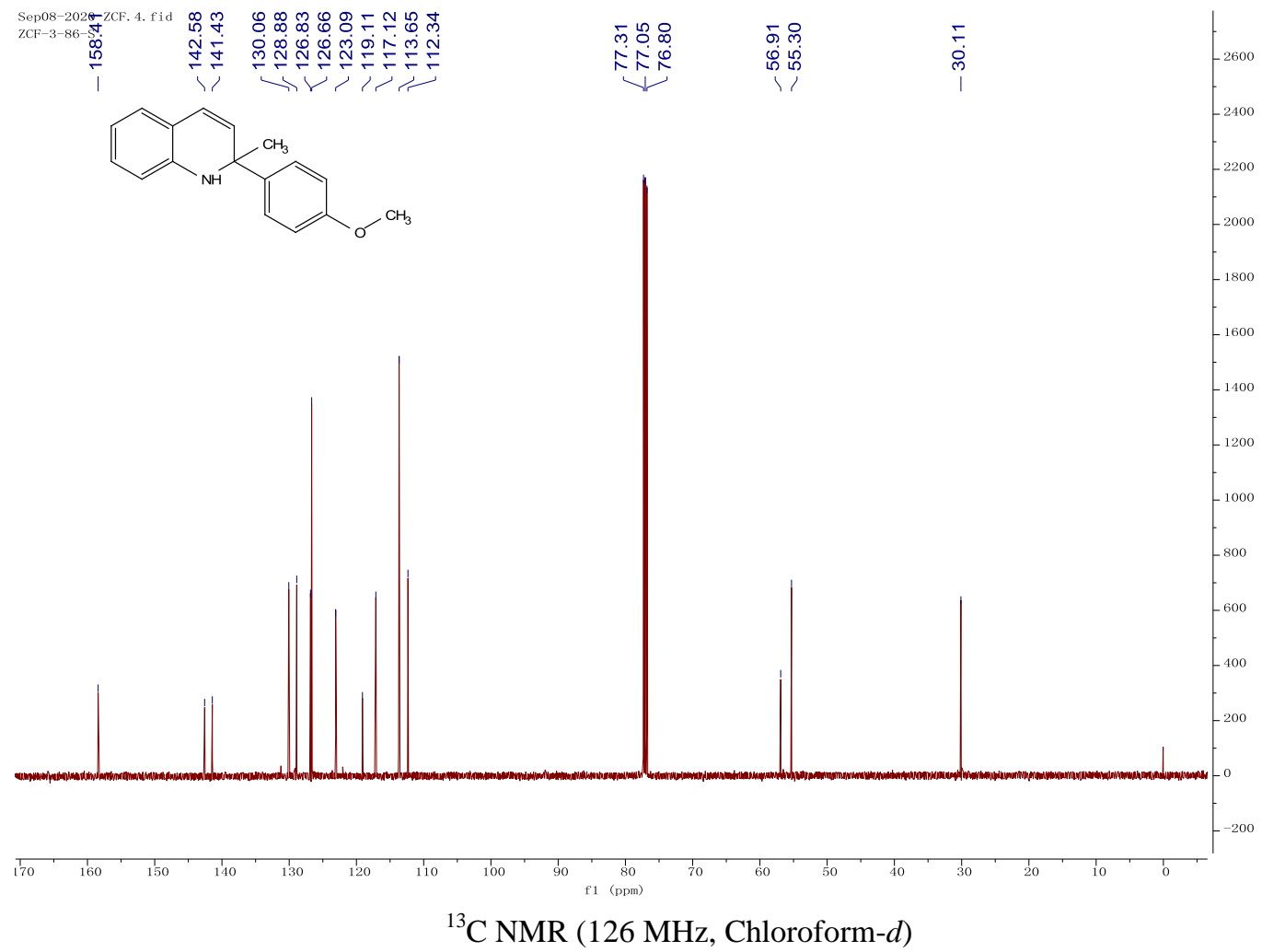


(S)-6-bromo-2-(4-methoxyphenyl)-2-methyl-1,2-dihydroquinoline ((S)-2e)
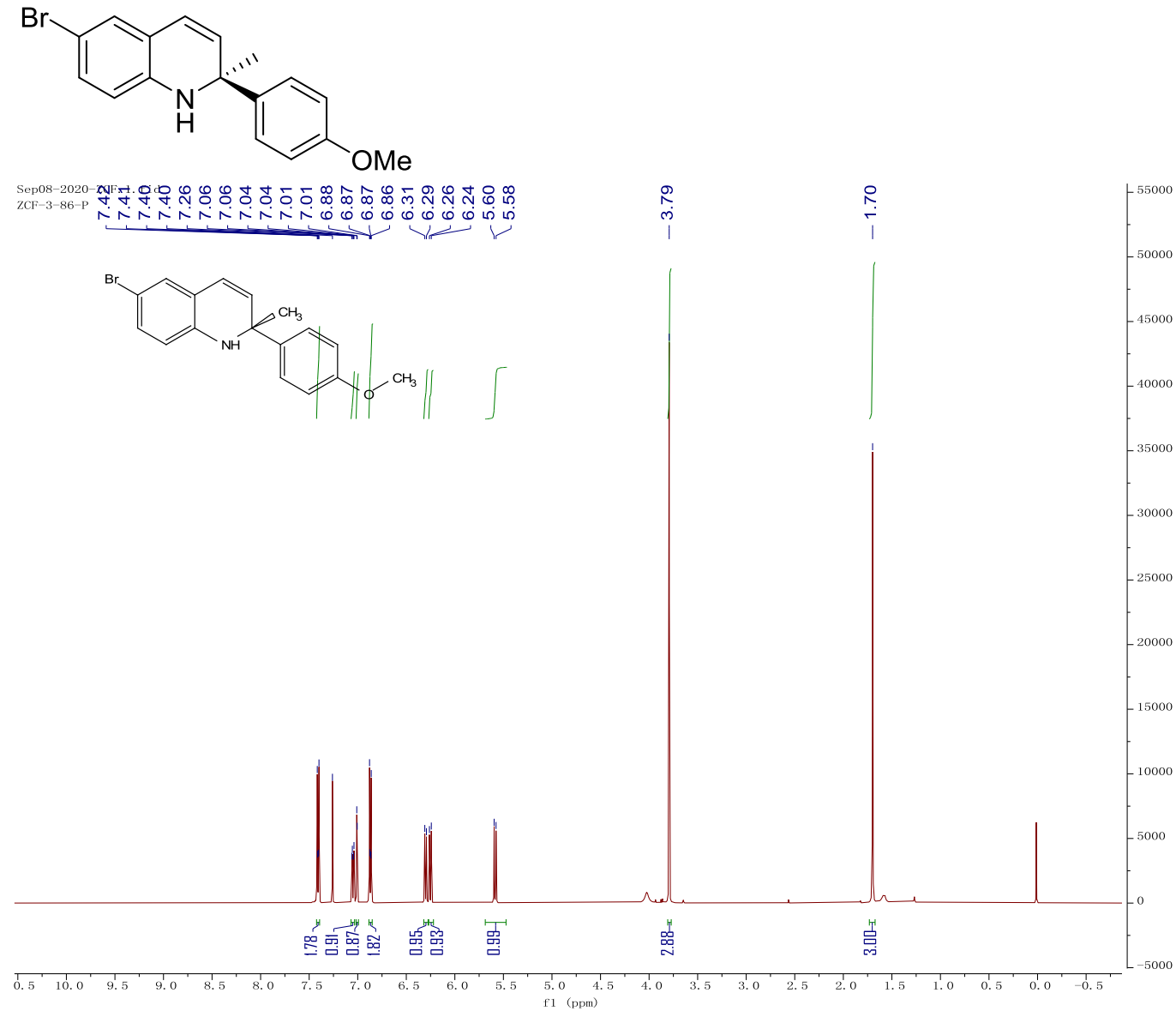

${ }^{1} \mathrm{H}$ NMR(500 MHz, Chloroform- $d$ )

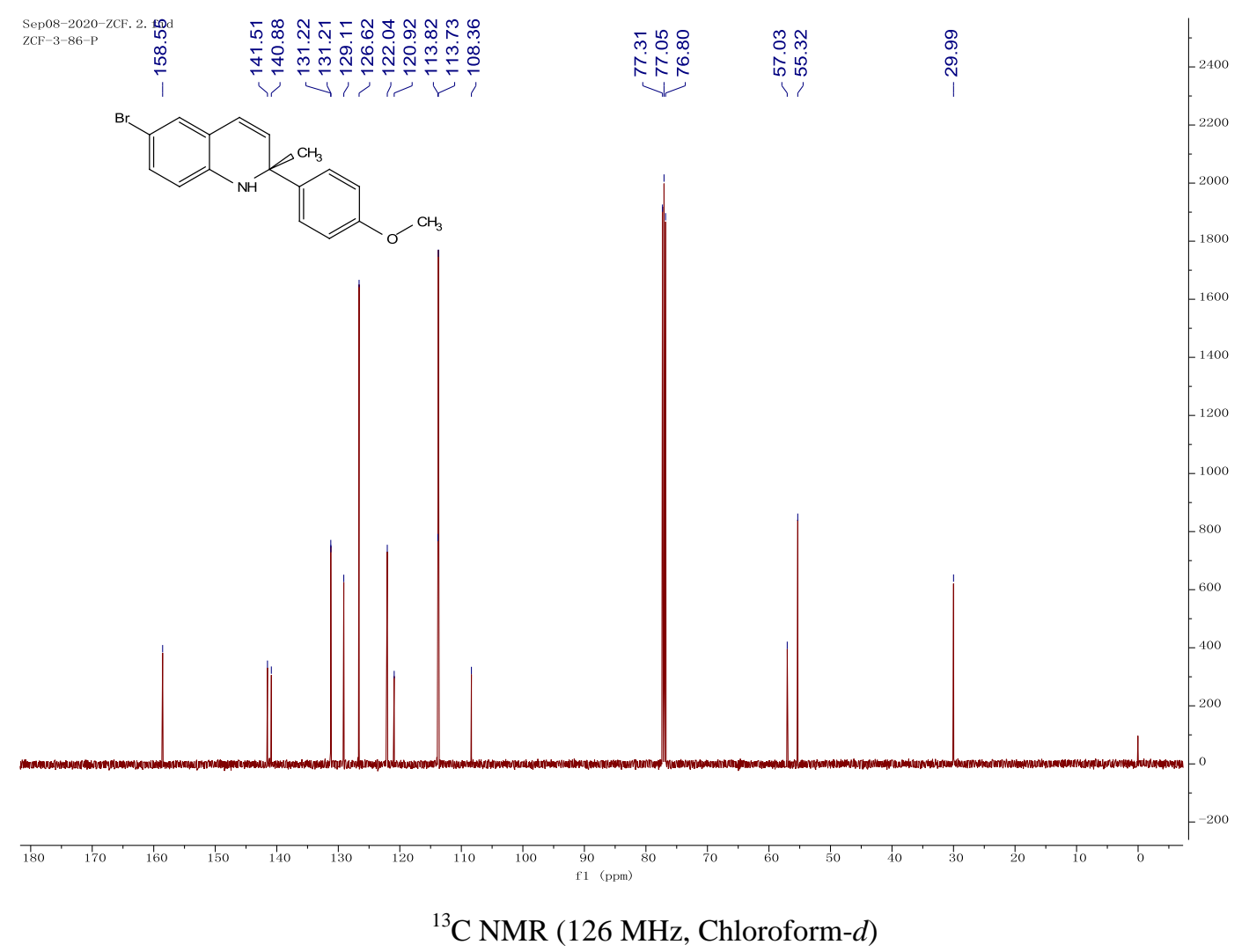


(R)-2-methyl-2-(m-tolyl)-1,2-dihydroquinoline ((R)-1f)<smiles>Cc1cccc([C@]2(C)C=Cc3ccccc3N2)c1</smiles>

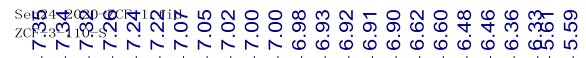
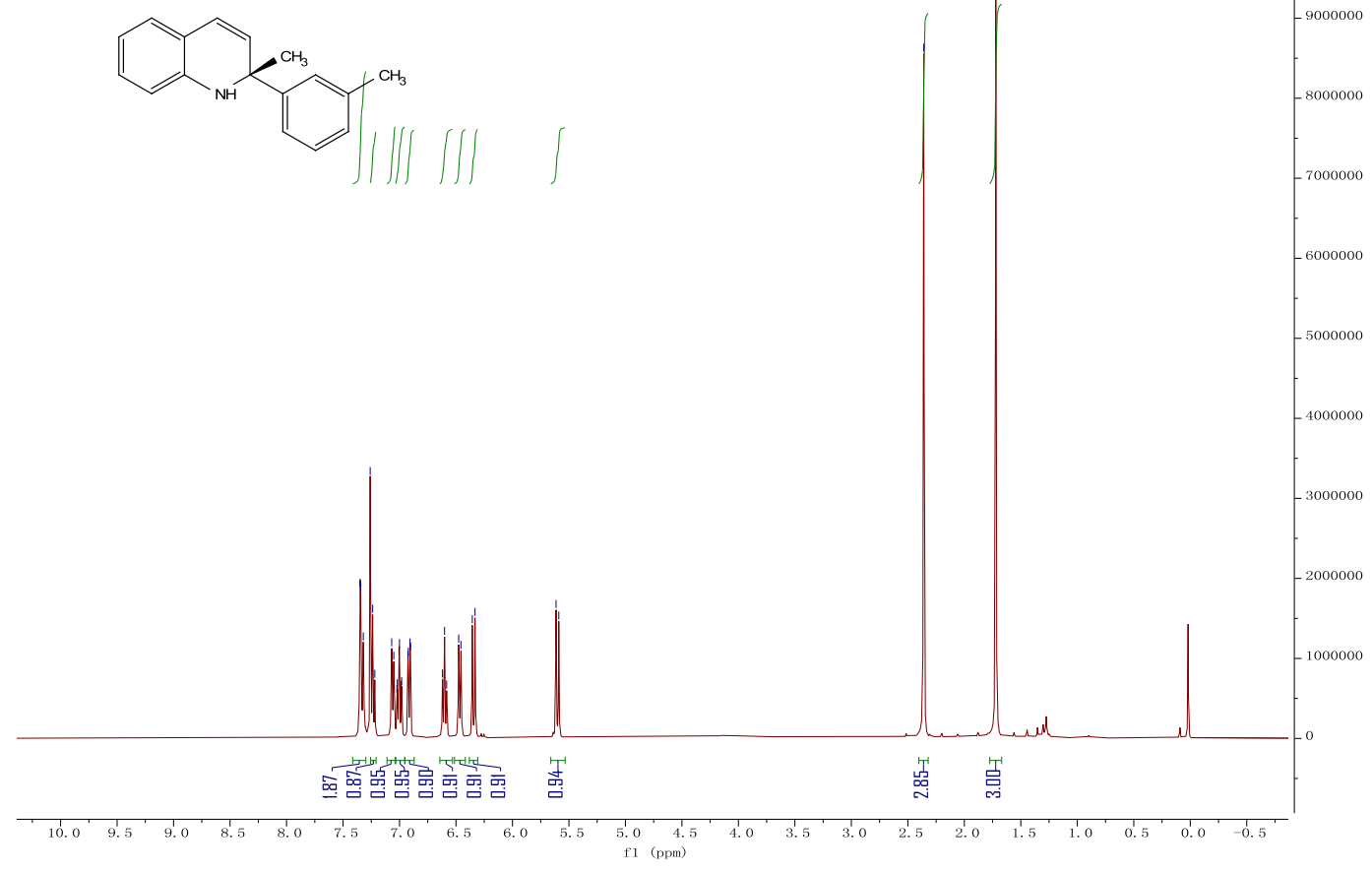

${ }^{1} \mathrm{H}$ NMR(400 MHz, Chloroform- $d$ )
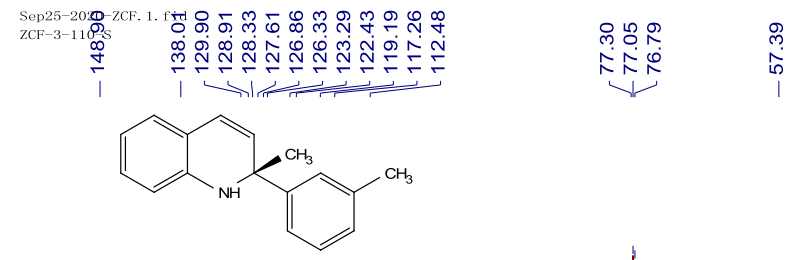

$\begin{array}{ll}0 & \hat{0} \\ \dot{0} & \bar{\kappa} \\ 1 & 1\end{array}$

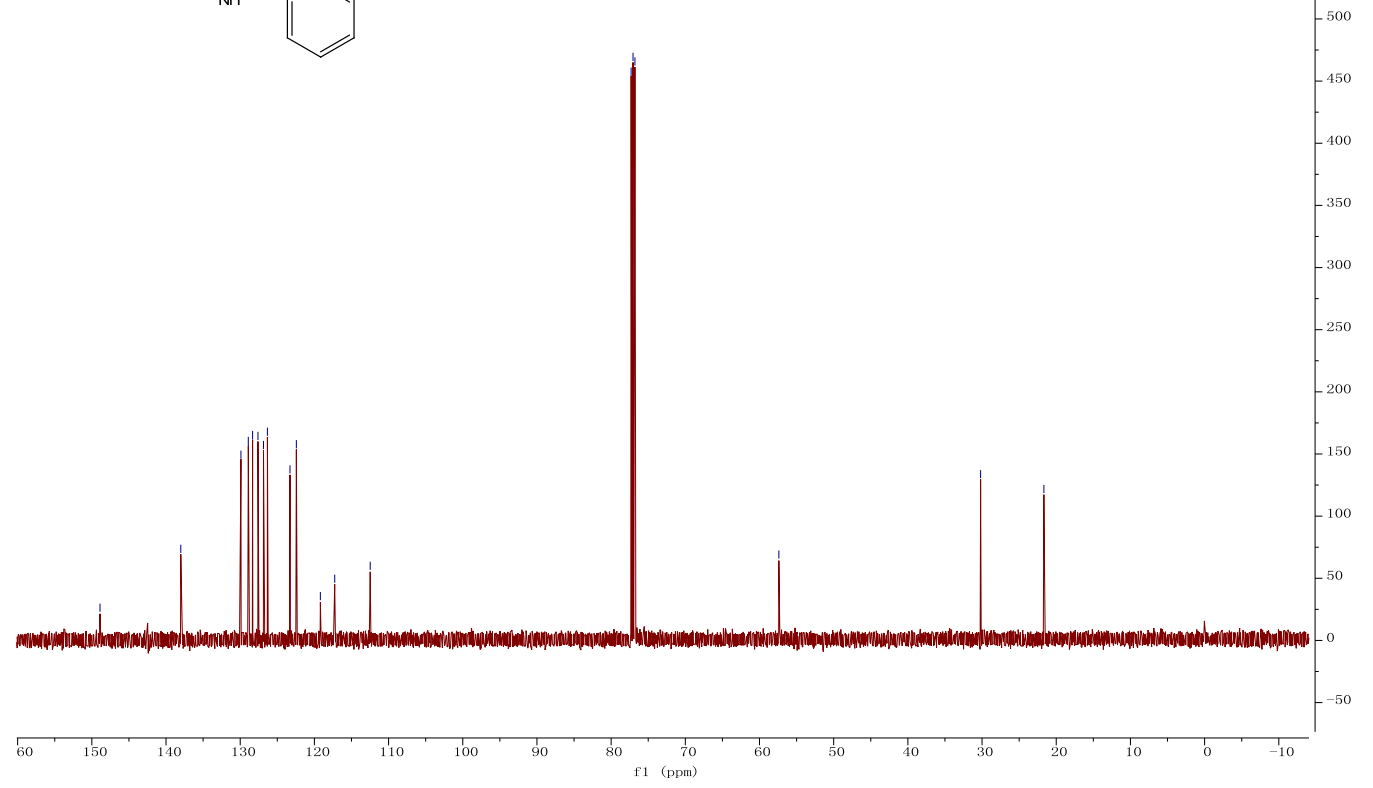

${ }^{13} \mathrm{C}$ NMR (126 MHz, Chloroform- $d$ ) 
(S)-6-bromo-2-methyl-2-(m-tolyl)-1,2-dihydroquinoline ((S)-2f)<smiles>Cc1cccc([C@]2(C)C=Cc3cc(Br)ccc3N2)c1</smiles>

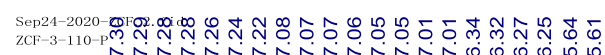

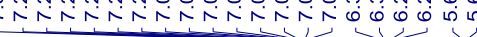<smiles>C#CC(C)(C)c1ccc(C2(C)C=Cc3cc(Br)ccc3N2)cc1</smiles>

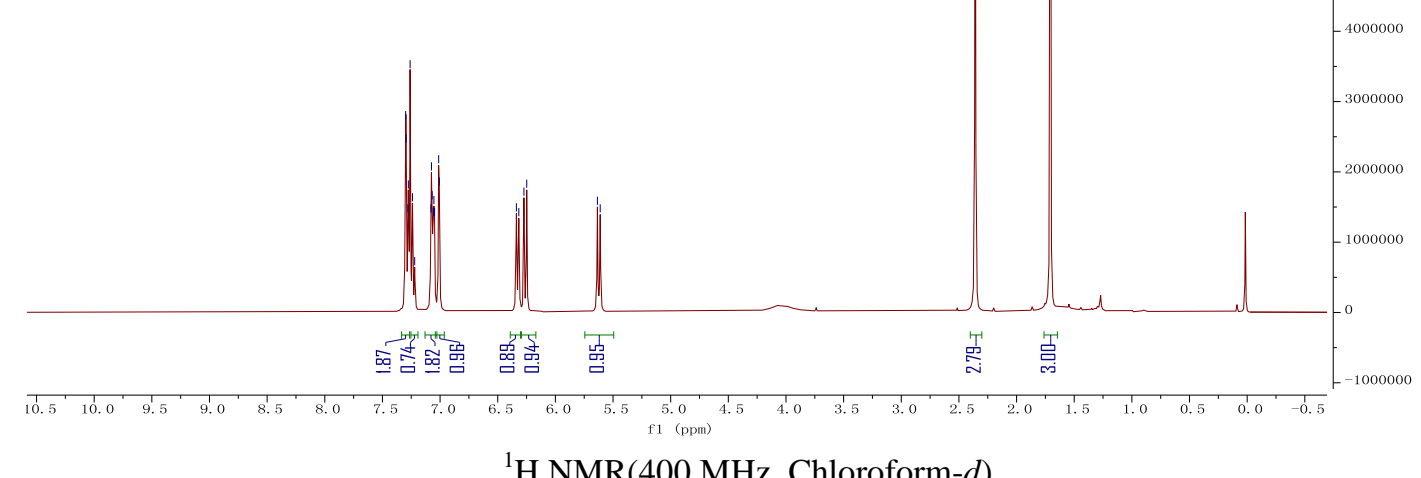

${ }^{1} \mathrm{H}$ NMR(400 MHz, Chloroform- $d$ )

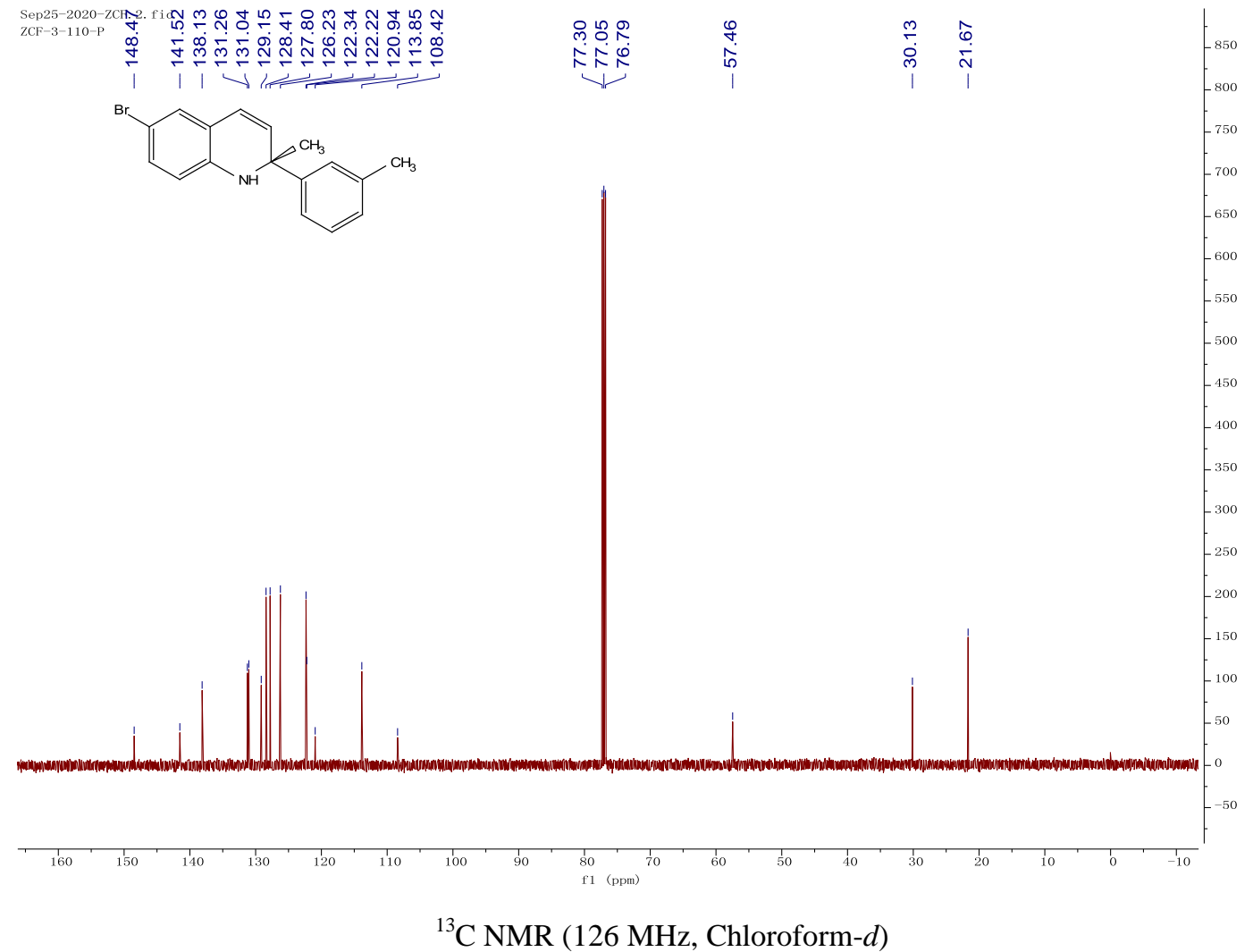


$(R)$-2-(3-methoxyphenyl)-2-methyl-1,2-dihydroquinoline $((R)-1 \mathbf{g})$<smiles>COc1cccc([C@]2(C)C=Cc3ccccc3N2)c1</smiles>

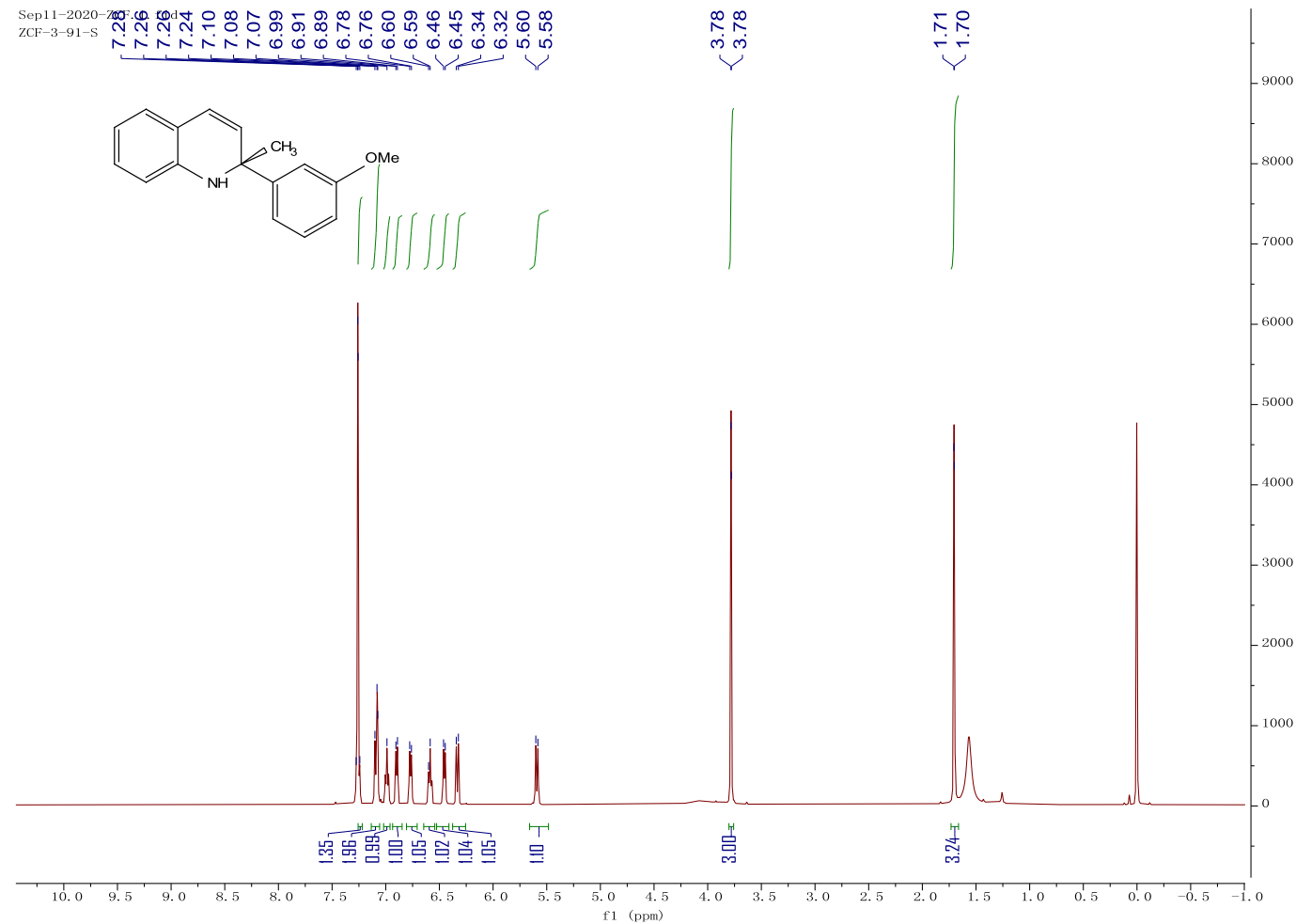

${ }^{1} \mathrm{H}$ NMR $(500 \mathrm{MHz}$, Chloroform- $d$ )

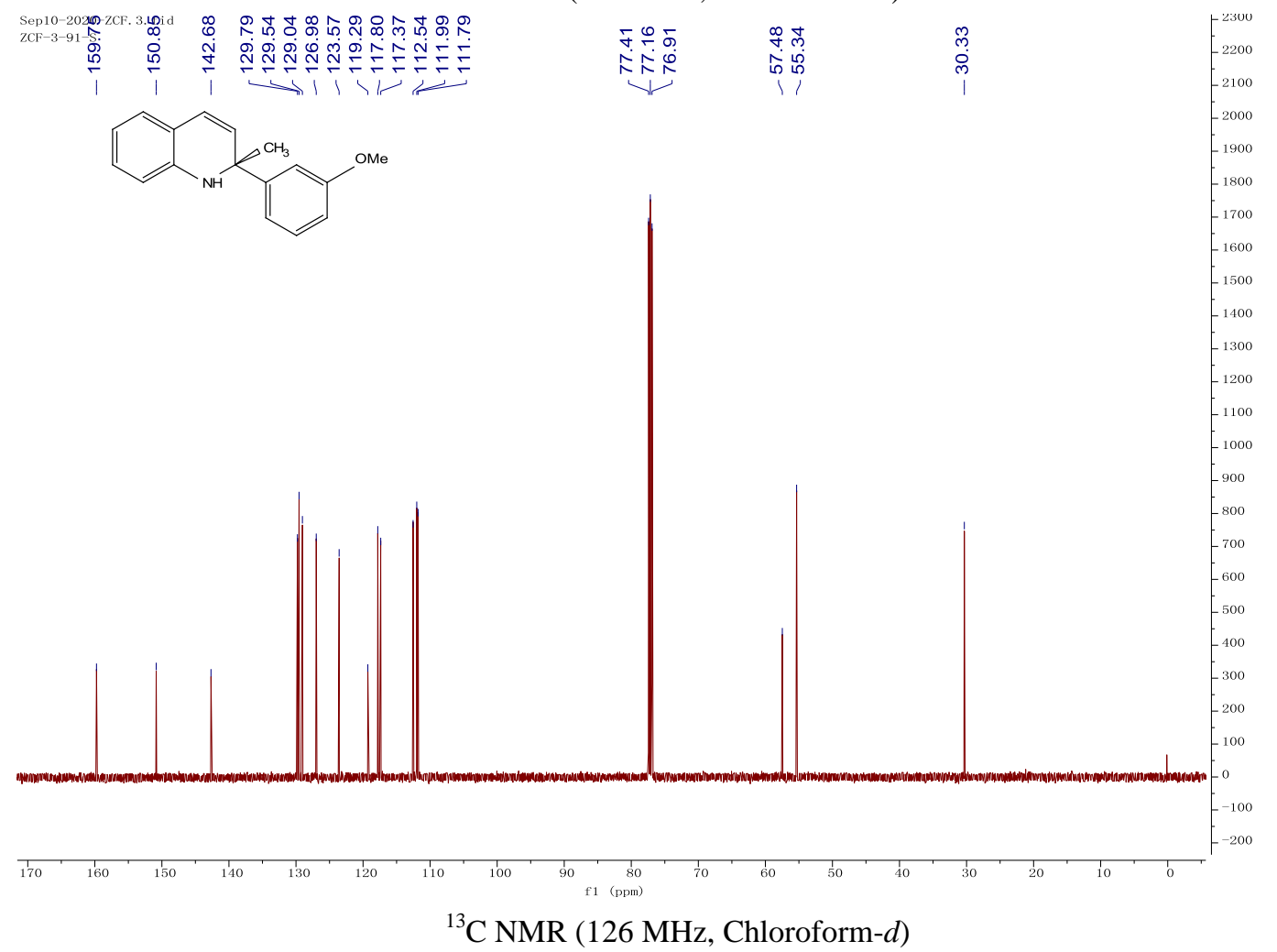


(S)-6-bromo-2-(3-methoxyphenyl)-2-methyl-1,2-dihydroquinoline ((S)-2g)<smiles>COc1cccc([C@]2(C)C=Cc3cc(Br)ccc3N2)c1</smiles>

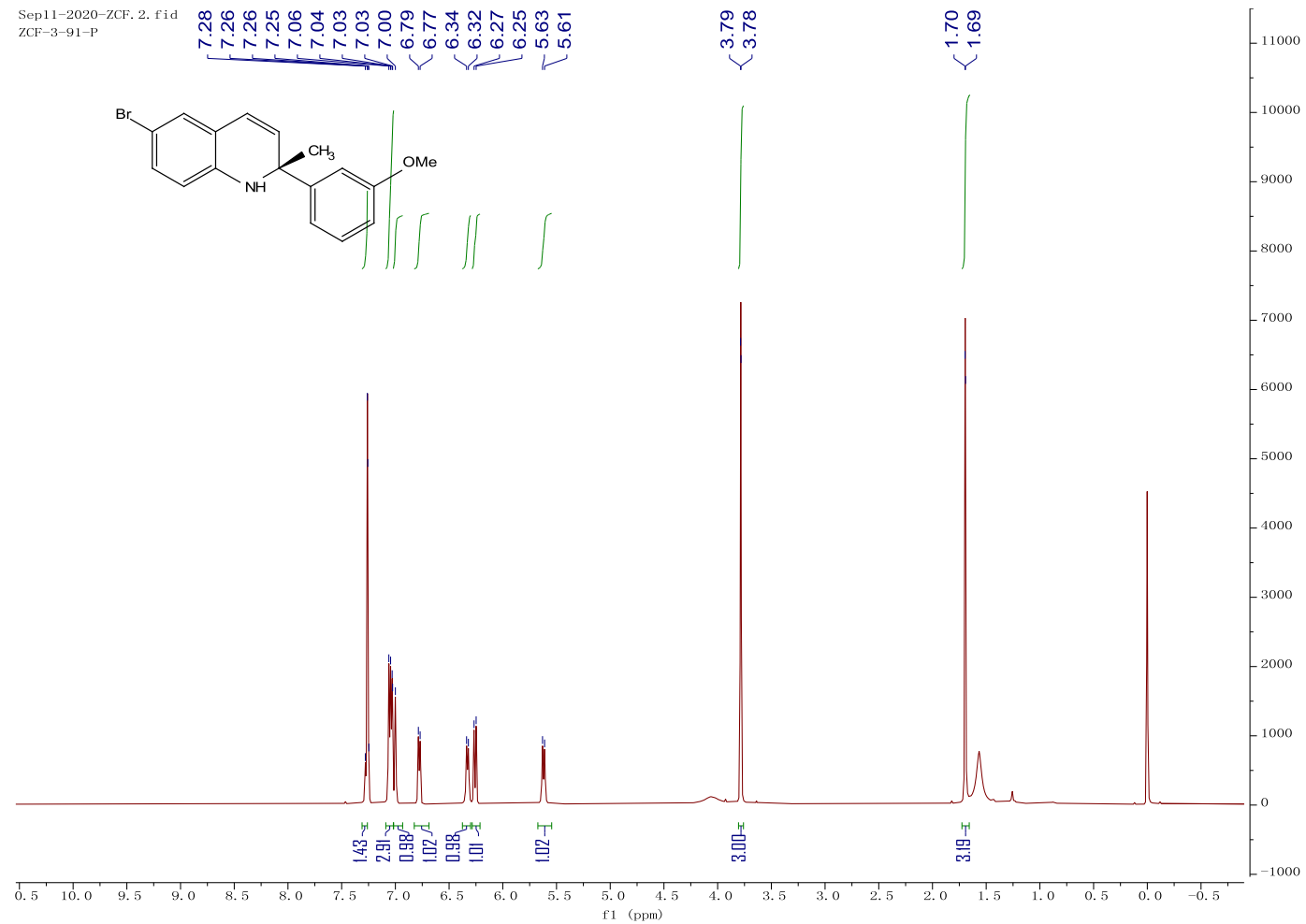

${ }^{1} \mathrm{H}$ NMR(500 MHz, Chloroform- $d$ )

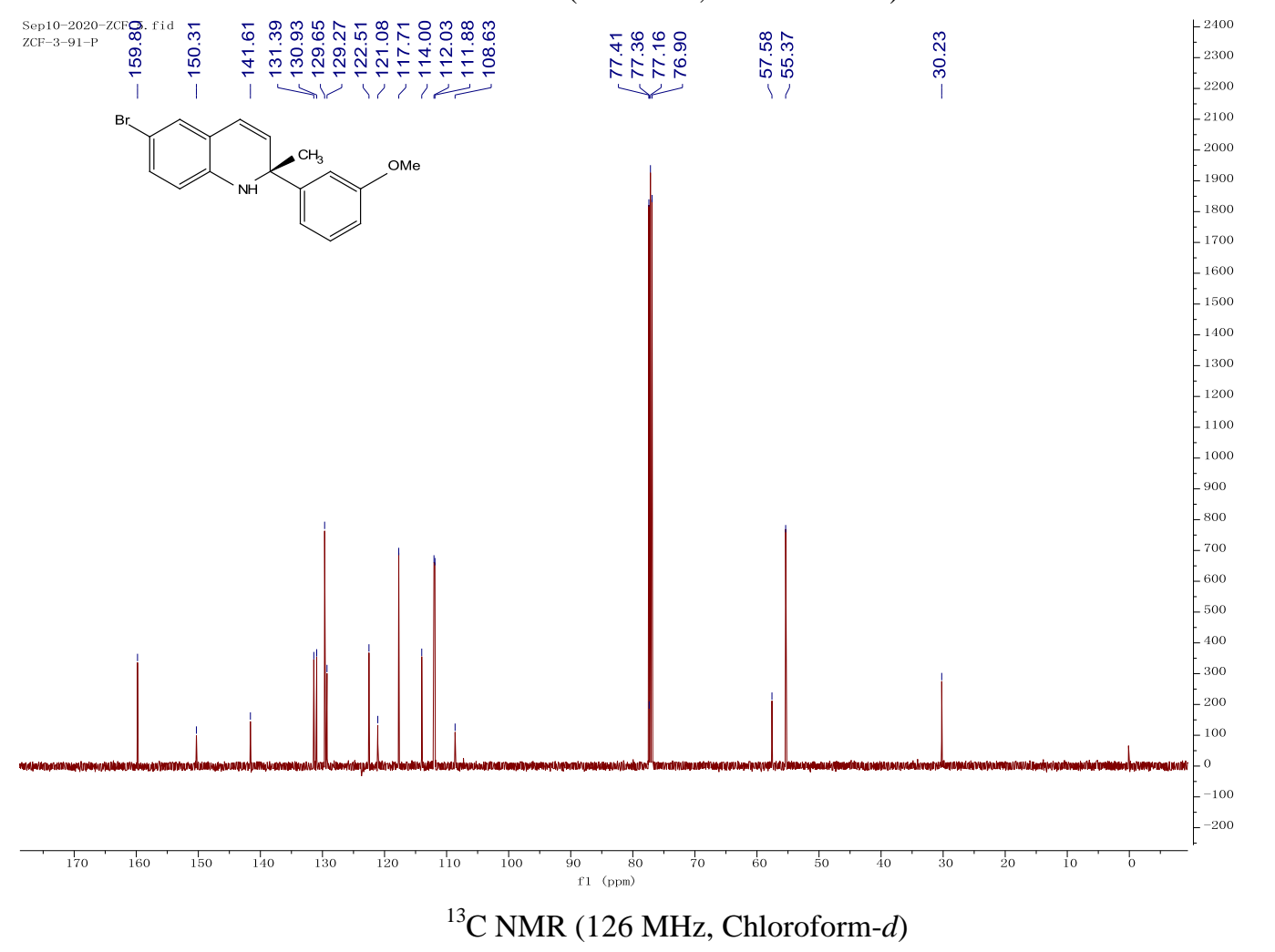


(R)-2-(3,5-dimethoxyphenyl)-2-methyl-1,2-dihydroquinoline $((R)$-1h)

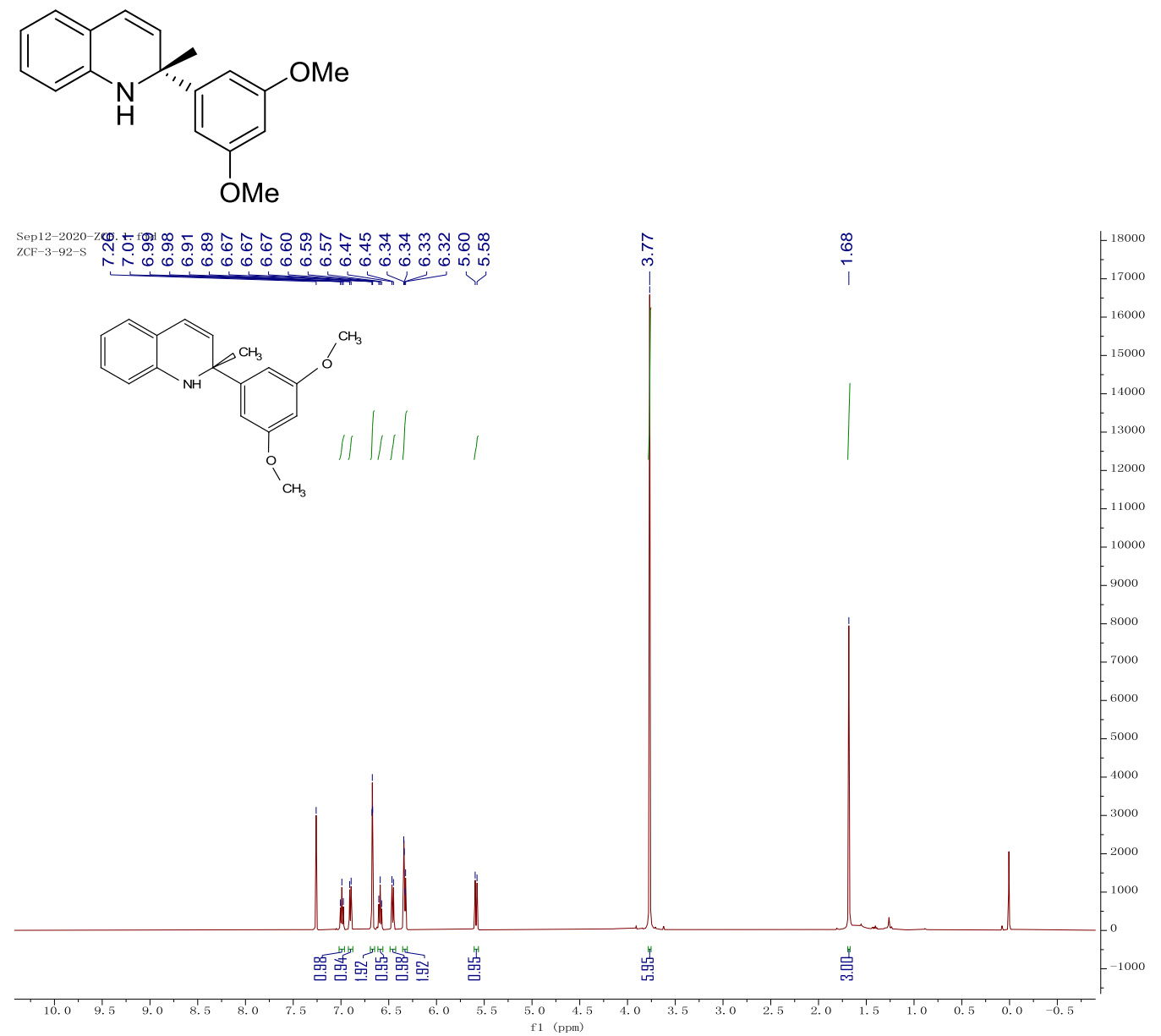

${ }^{1} \mathrm{H}$ NMR(500 MHz, Chloroform- $d$ )

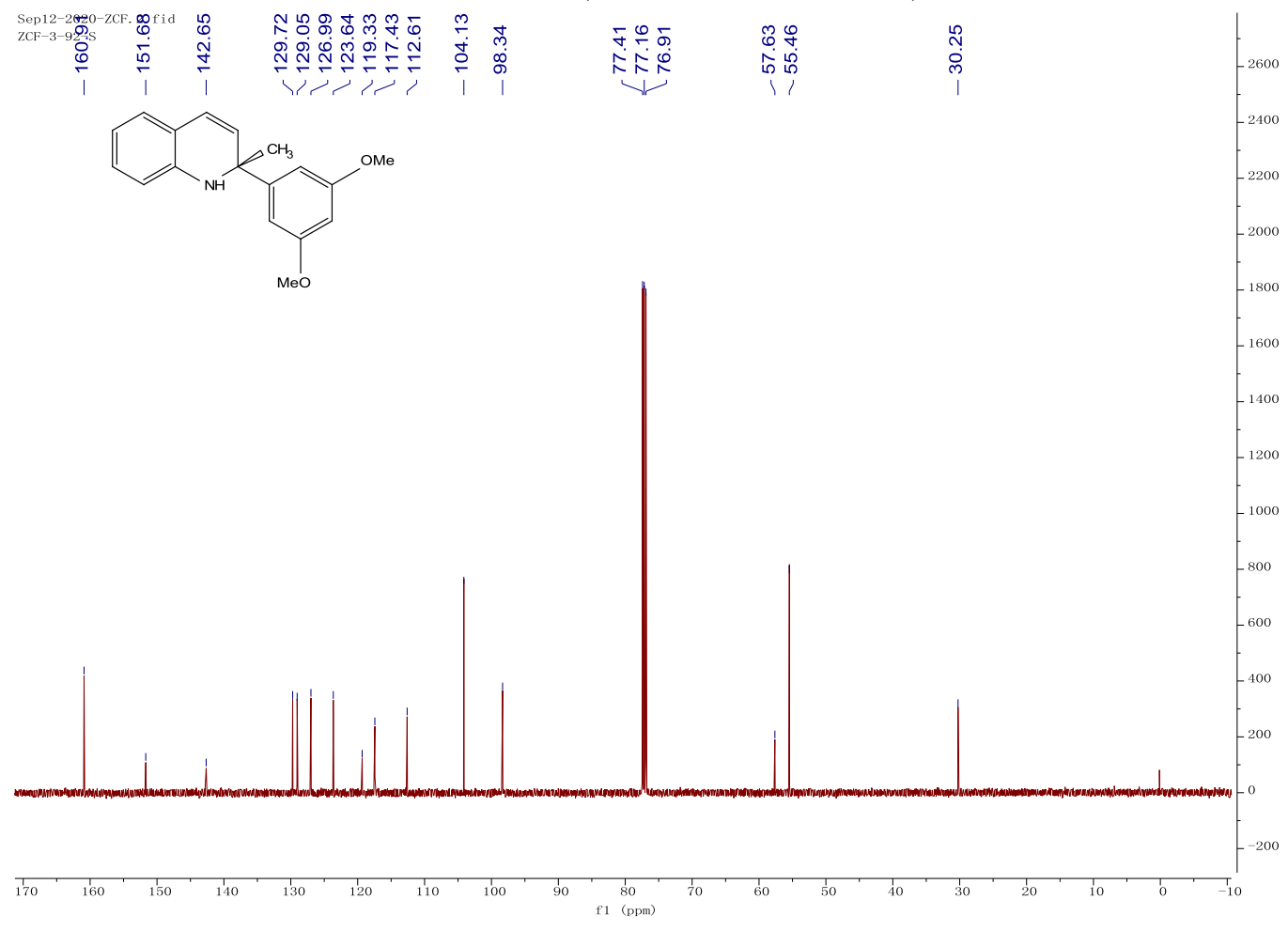

${ }^{13} \mathrm{C}$ NMR (126 MHz, Chloroform- $d$ ) 
(S)-6-bromo-2-(3,5-dimethoxyphenyl)-2-methyl-1,2-dihydroquinoline ((R)-2h)<smiles>COc1cc(OC)cc([C@]2(C)C=Cc3cc(Br)ccc3N2)c1</smiles>

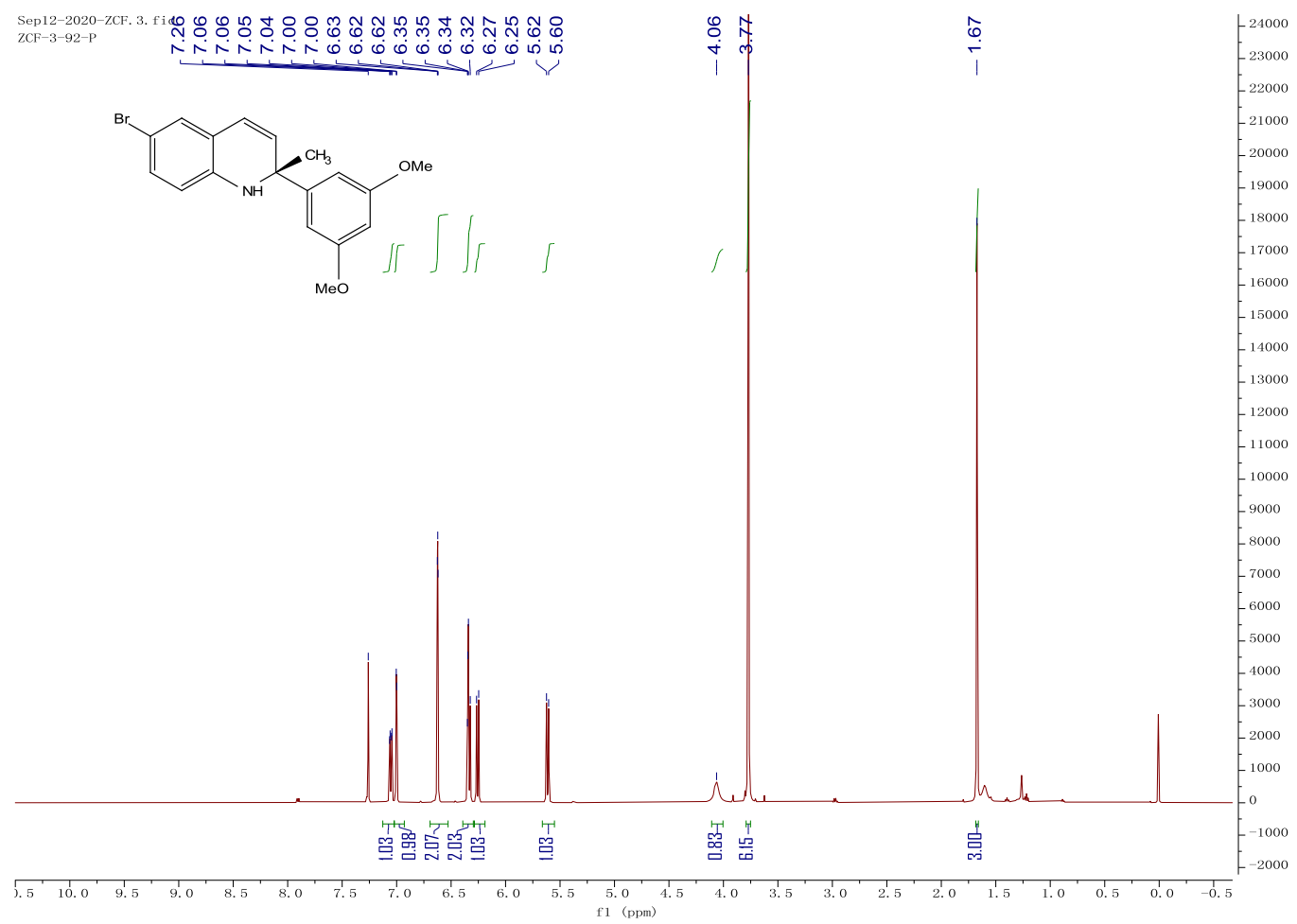

${ }^{1} \mathrm{H}$ NMR(500 MHz, Chloroform- $d$ )

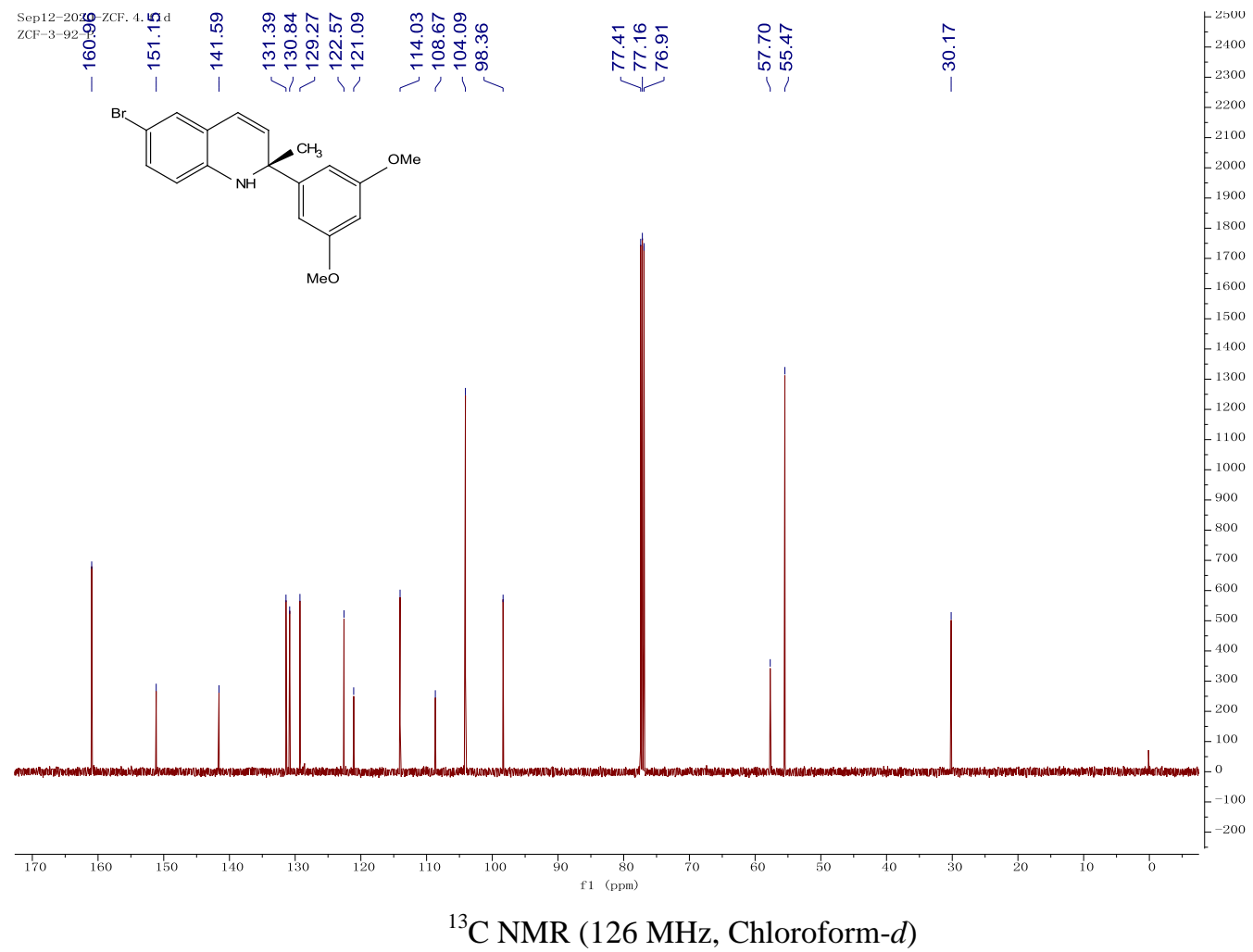


$(R)$-2-(benzo[d][1,3]dioxol-5-yl)-2-methyl-1,2-dihydroquinoline $((R)-1 \mathbf{i})$<smiles>C[C@]1(c2ccc3c(c2)OCO3)C=Cc2ccccc2N1</smiles>

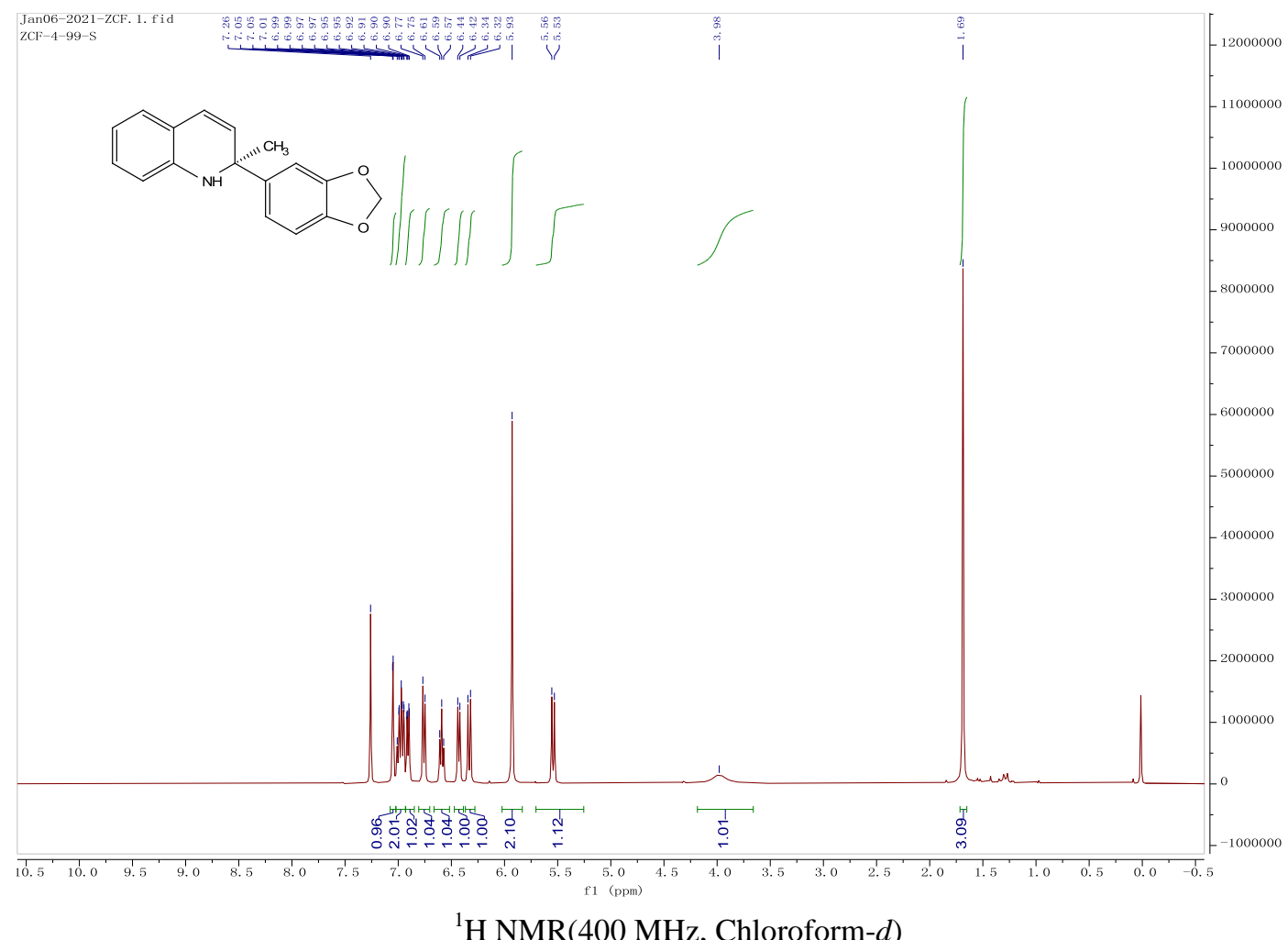

${ }^{1} \mathrm{H}$ NMR(400 MHz, Chloroform- $d$ )

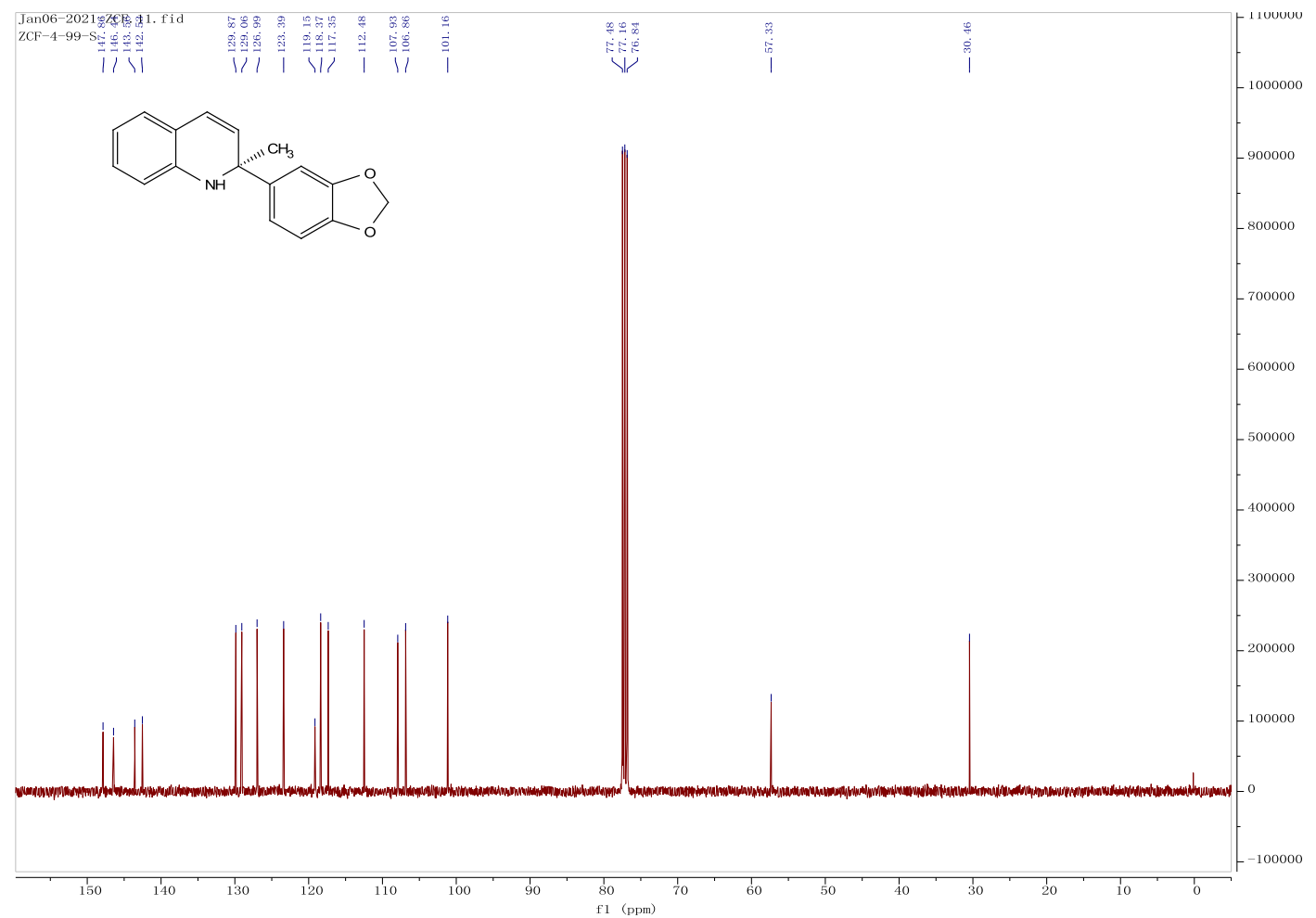

${ }^{13} \mathrm{C}$ NMR (101 MHz, Chloroform- $d$ ) 
(S)-2-(benzo[d][1,3]dioxol-5-yl)-6-bromo-2-methyl-1,2-dihydroquinoline ((S)-2i)

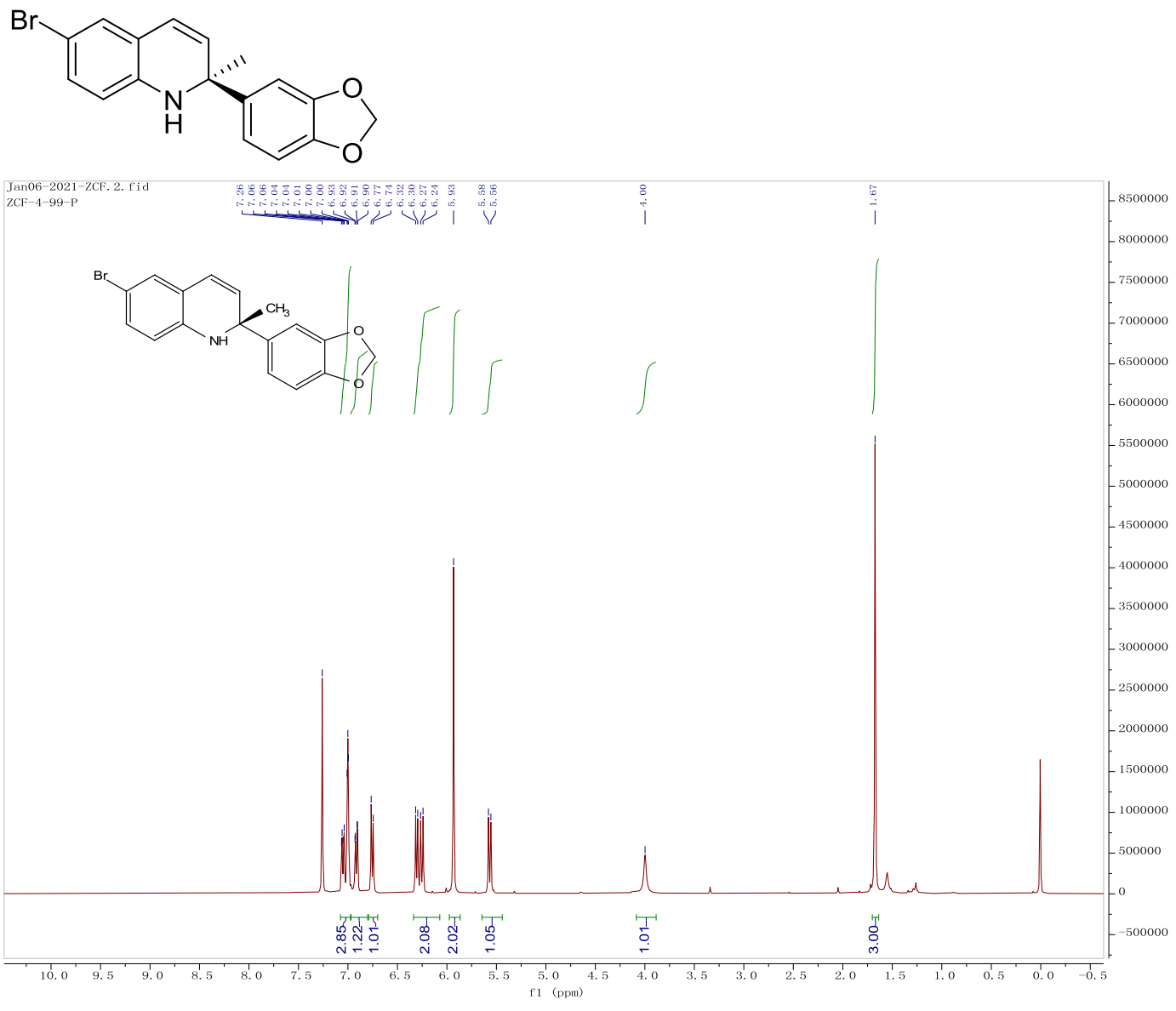

${ }^{1} \mathrm{H}$ NMR(400 MHz, Chloroform- $d$ )

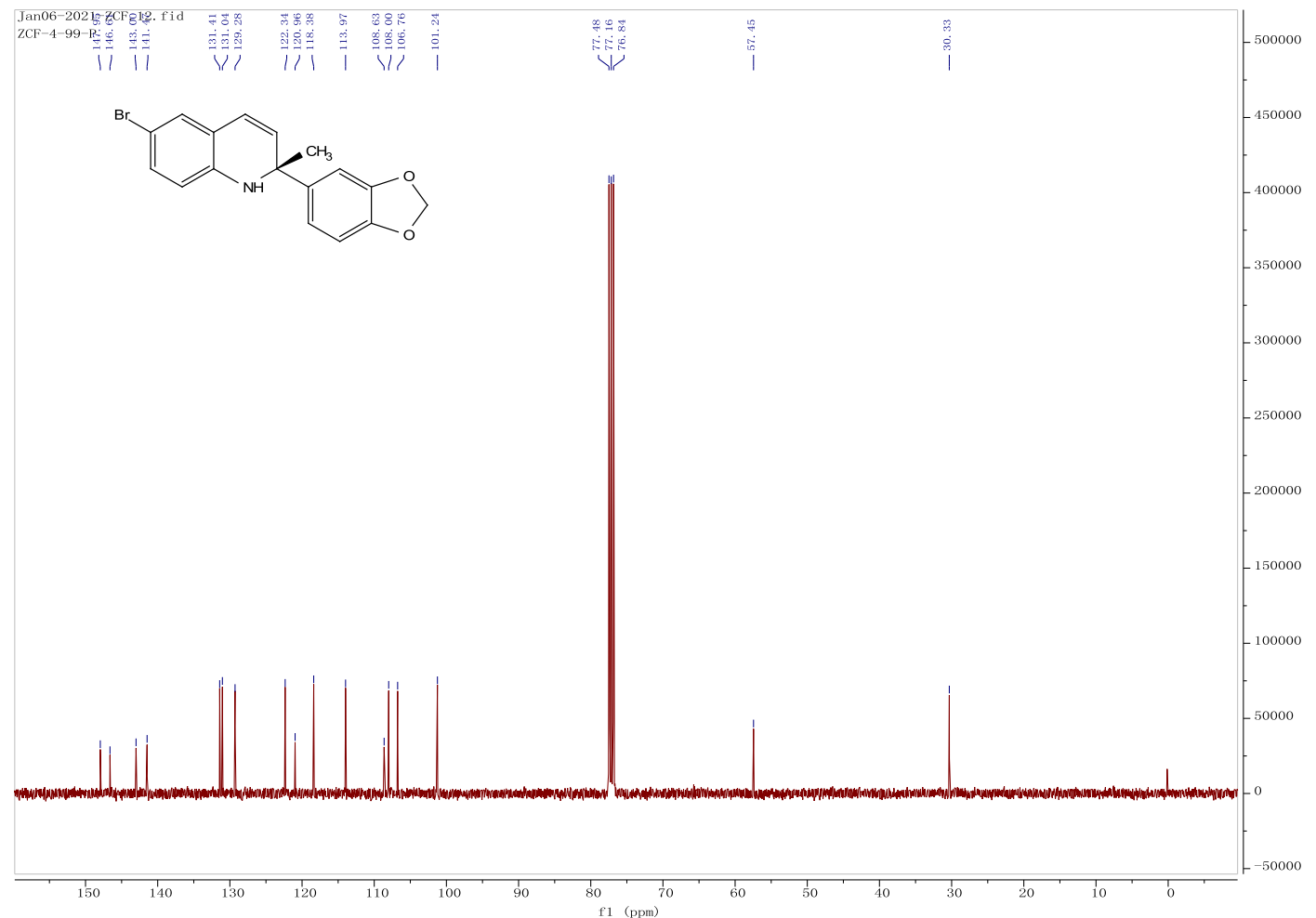

${ }^{13} \mathrm{C}$ NMR (101 MHz, Chloroform- $d$ ) 
$(R)$-2-methyl-2-(thiophen-3-yl)-1,2-dihydroquinoline $((R)-\mathbf{1} \mathbf{j})$<smiles>CC1(c2ccsc2)C=Cc2ccccc2N1</smiles>

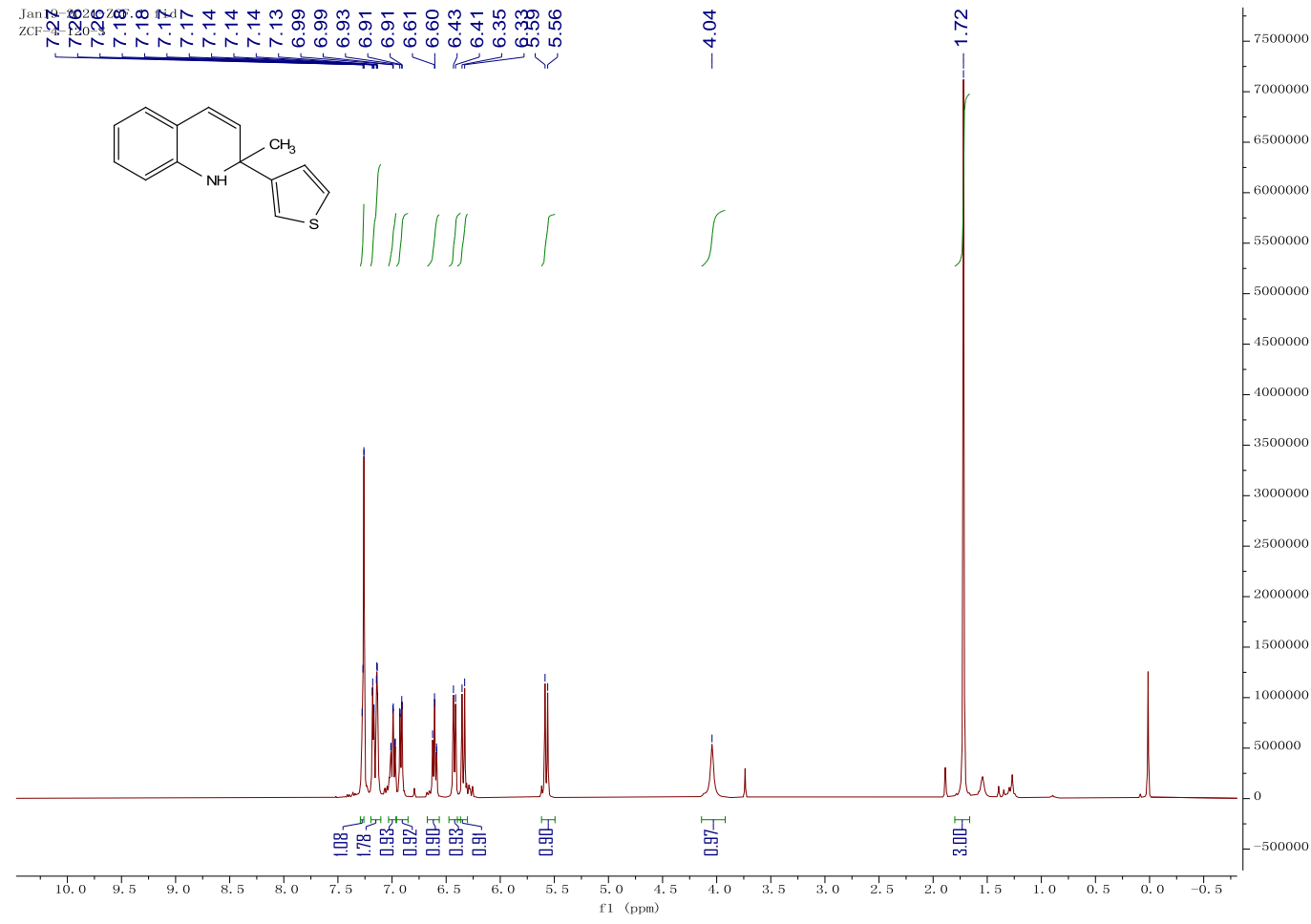

${ }^{1} \mathrm{H}$ NMR(400 MHz, Chloroform- $d$ )

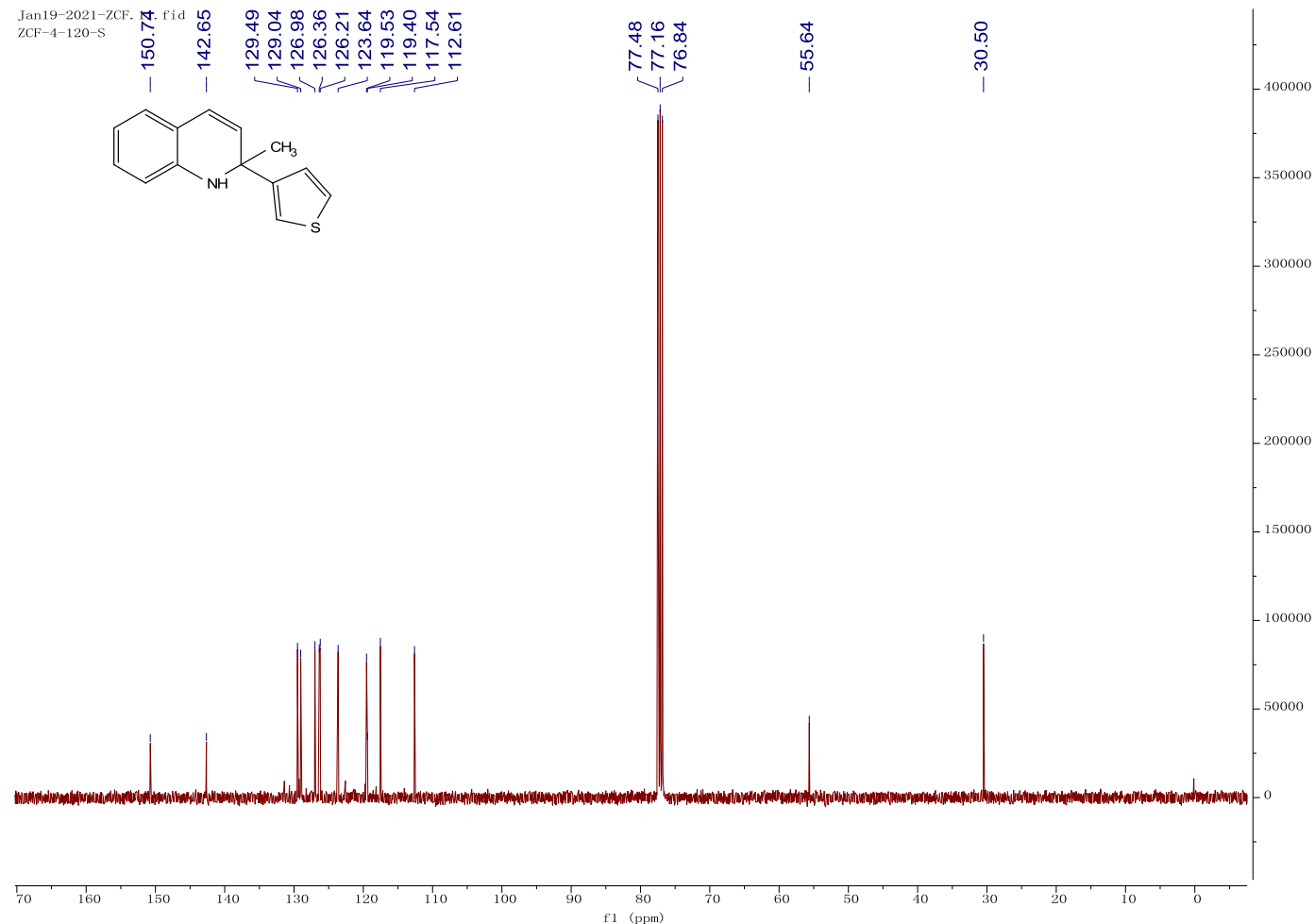

${ }^{13} \mathrm{C}$ NMR (101 MHz, Chloroform- $d$ ) 
(S)-6-bromo-2-methyl-2-(thiophen-3-yl)-1,2-dihydroquinoline ((S)-2j)<smiles>C[C@]1(c2ccsc2)C=Cc2cc(Br)ccc2N1</smiles>

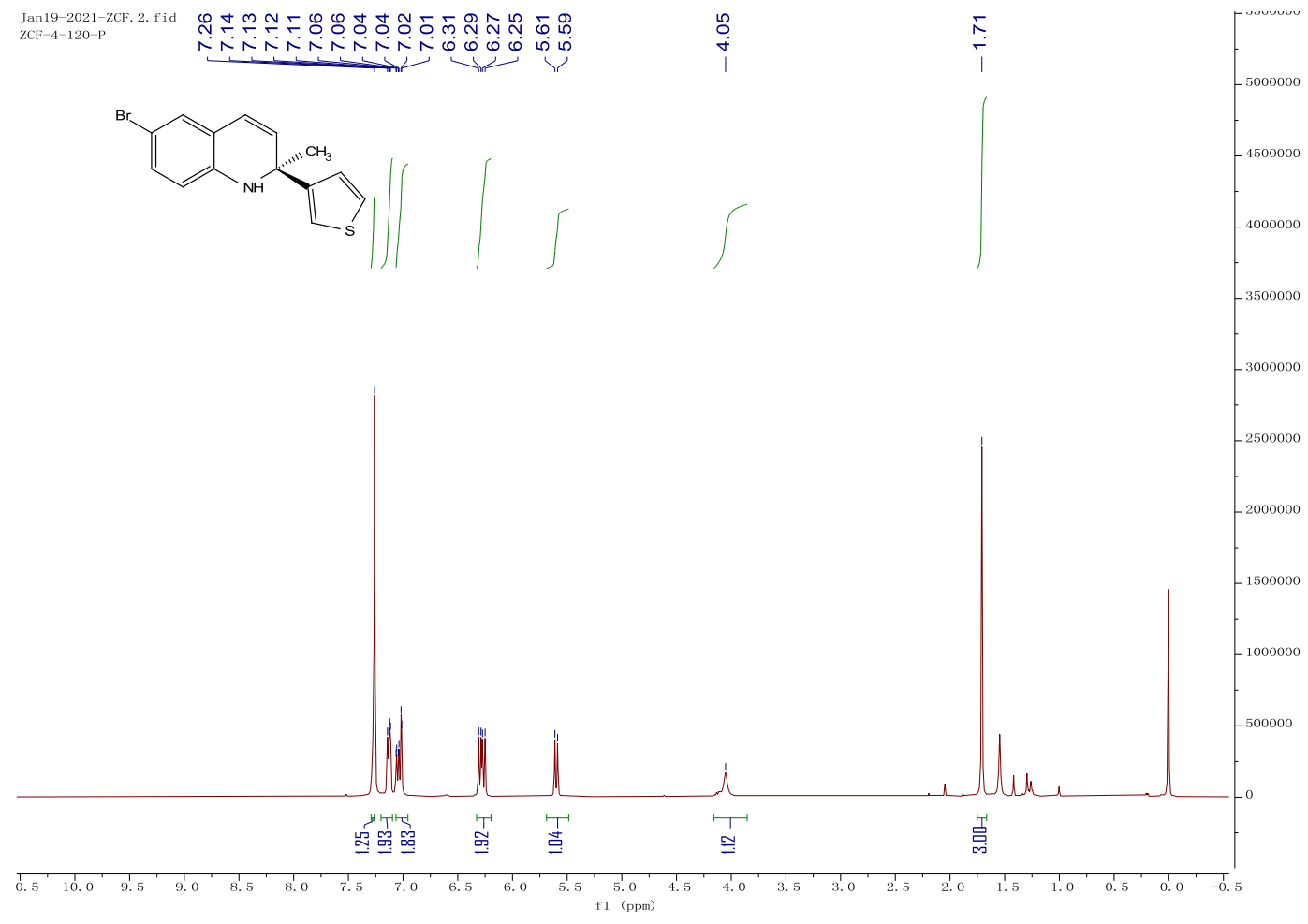

${ }^{1} \mathrm{H}$ NMR(400 MHz, Chloroform- $d$ )

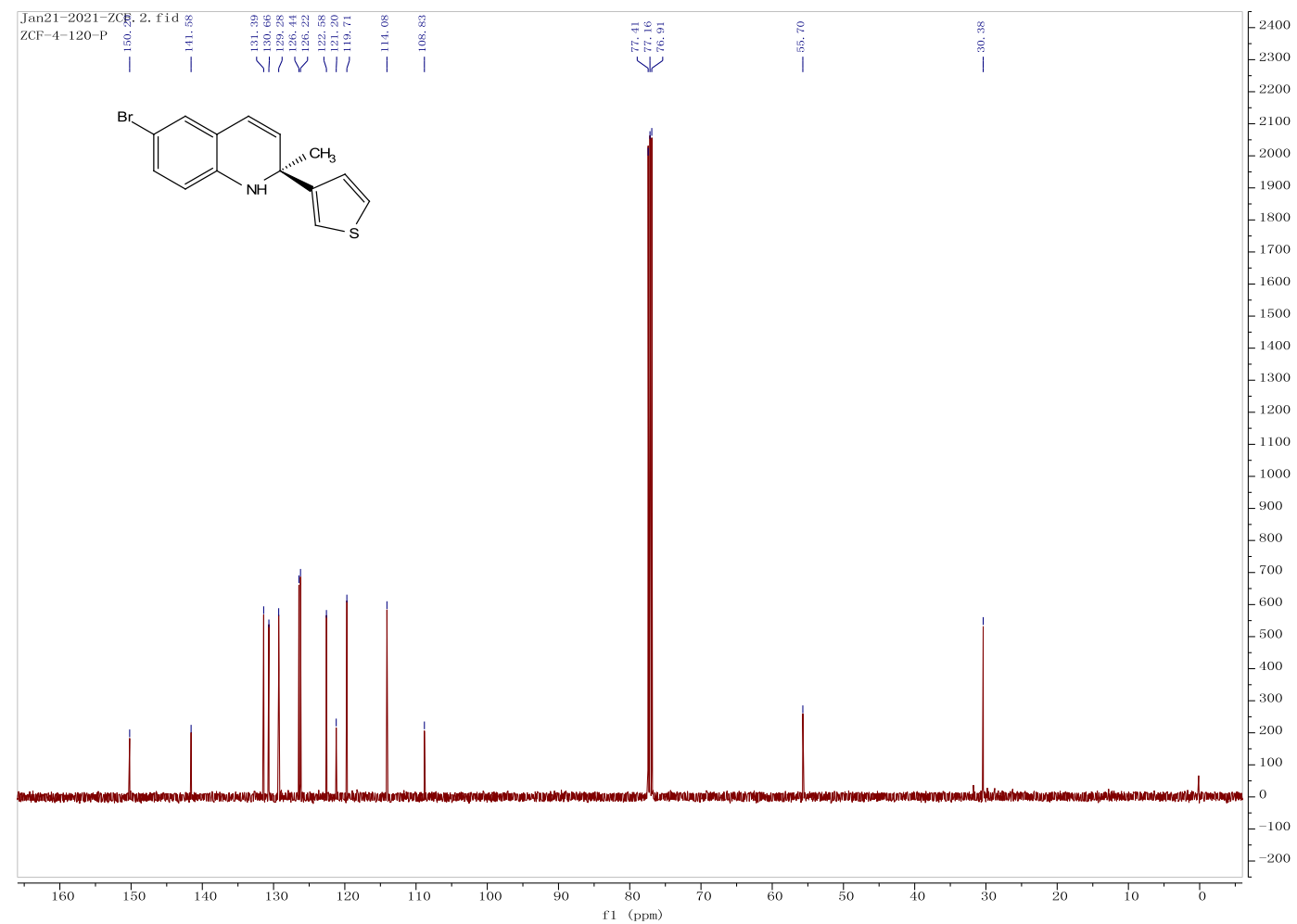

${ }^{13} \mathrm{C}$ NMR (126 MHz, Chloroform- $d$ ) 
(R)-2-(furan-3-yl)-2-methyl-1,2-dihydroquinoline $((R)-\mathbf{1 k})$<smiles>CC1(c2ccoc2)C=Cc2ccccc2N1</smiles>

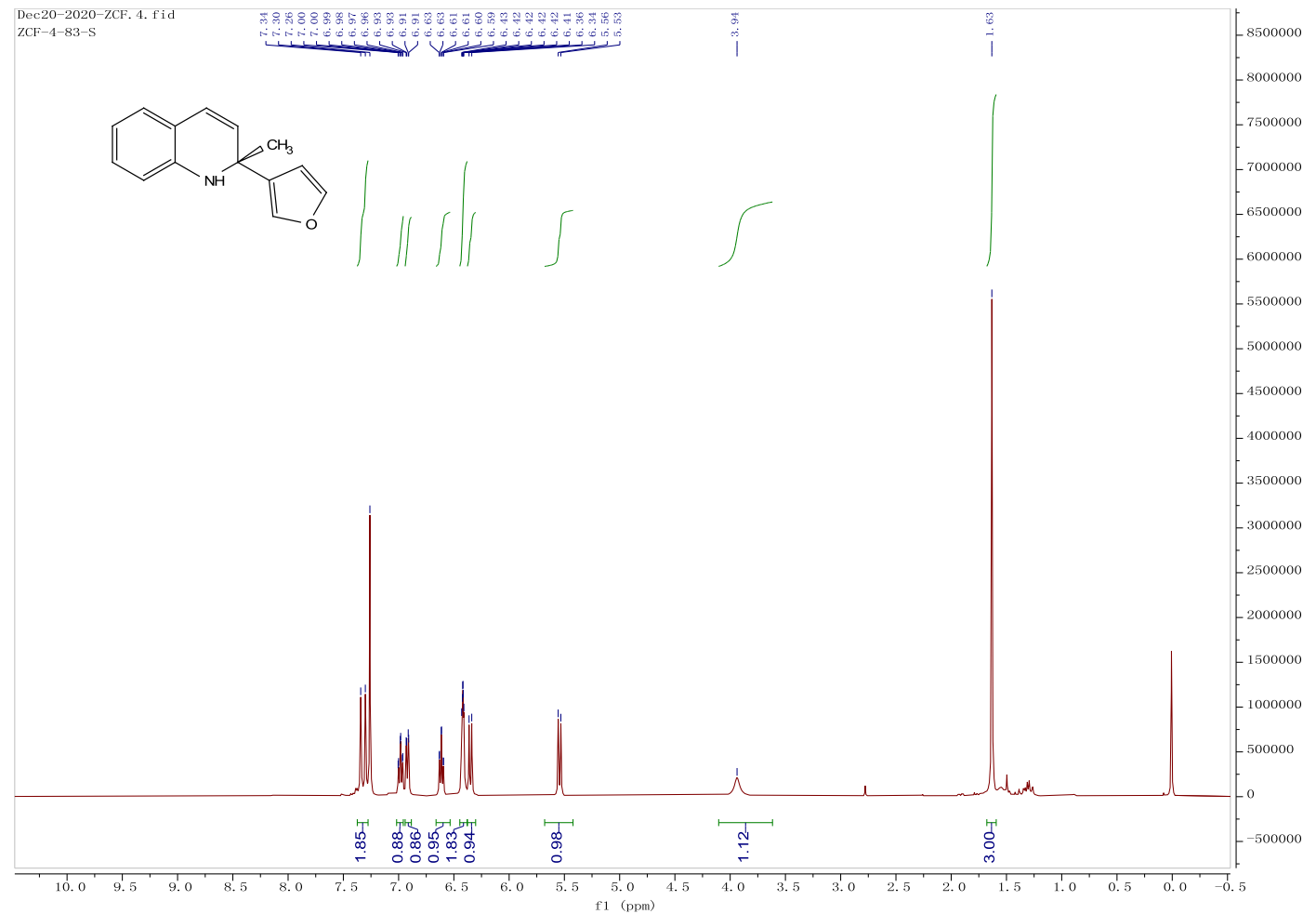

${ }^{1} \mathrm{H}$ NMR(400 MHz, Chloroform- $d$ )

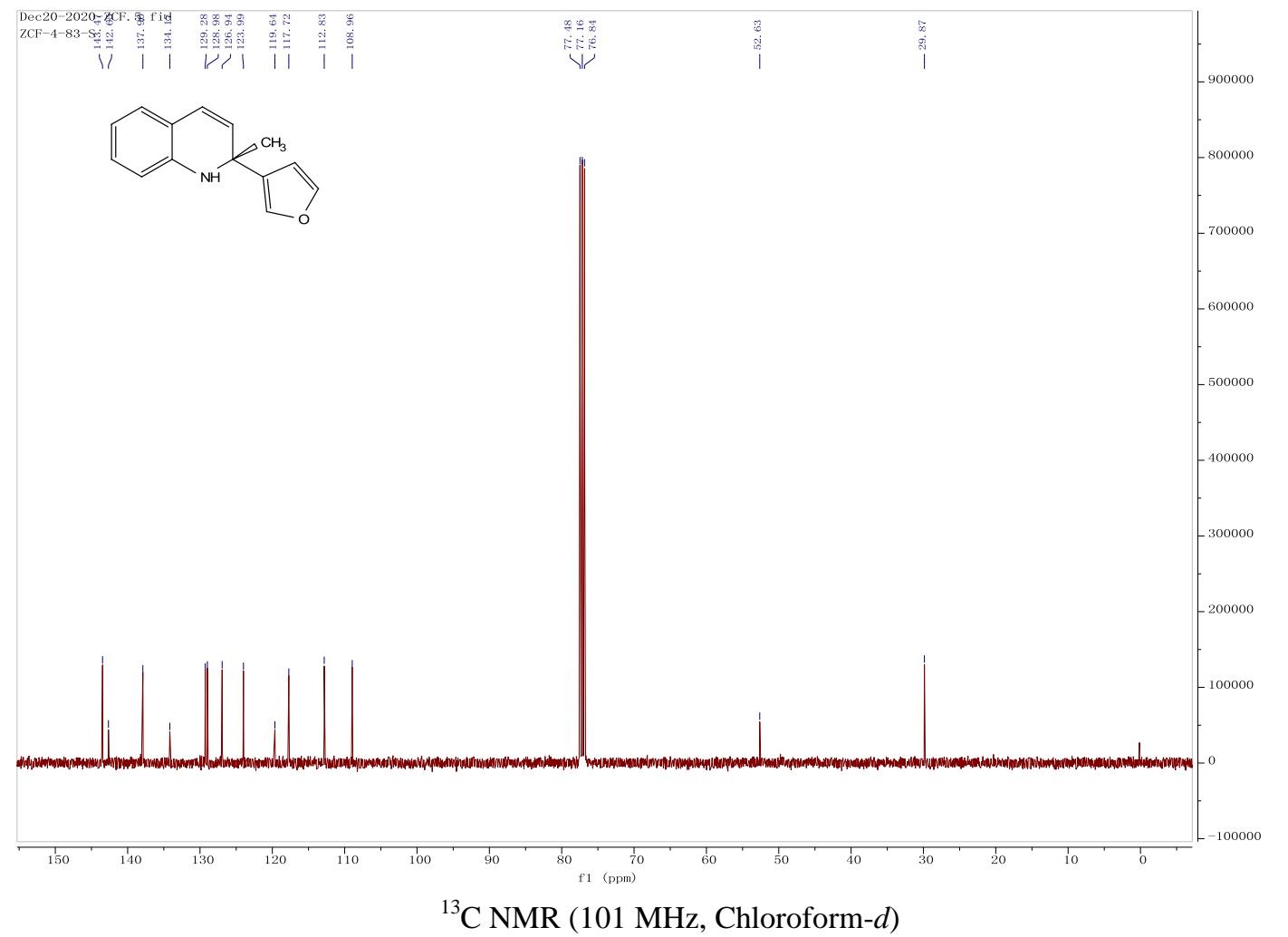


(S)-6-bromo-2-(furan-3-yl)-2-methyl-1,2-dihydroquinoline ((S)-2k)<smiles>C[C@]1(c2ccoc2)C=Cc2cc(Br)ccc2N1</smiles>

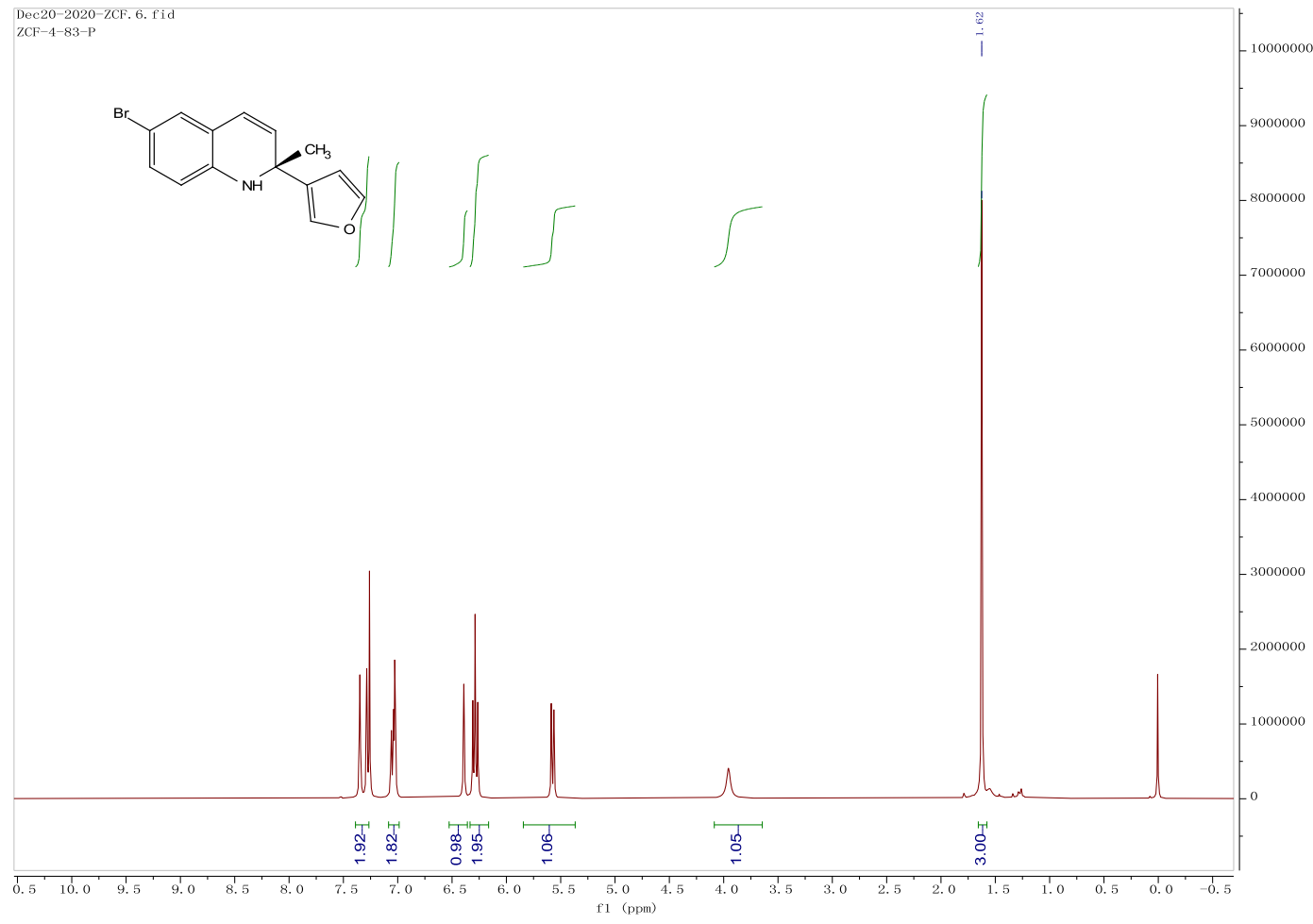

${ }^{1} \mathrm{H}$ NMR(400 MHz, Chloroform- $d$ )

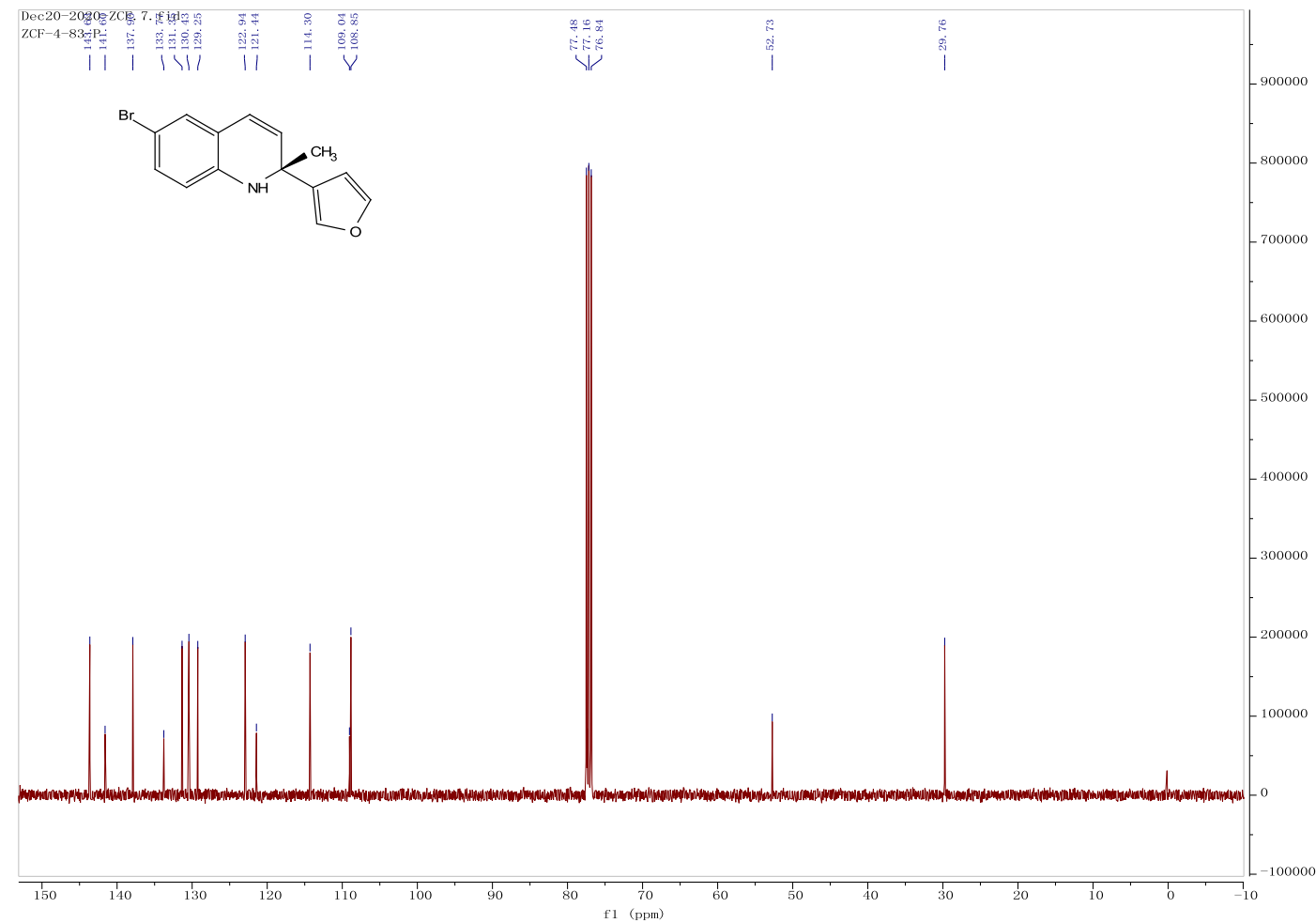

${ }^{13} \mathrm{C}$ NMR (101 MHz, Chloroform- $d$ ) 
(R)-2-ethyl-2-phenyl-1,2-dihydroquinoline ((R)-11)<smiles>CC[C@@]1(c2ccccc2)C=Cc2ccccc2N1</smiles>

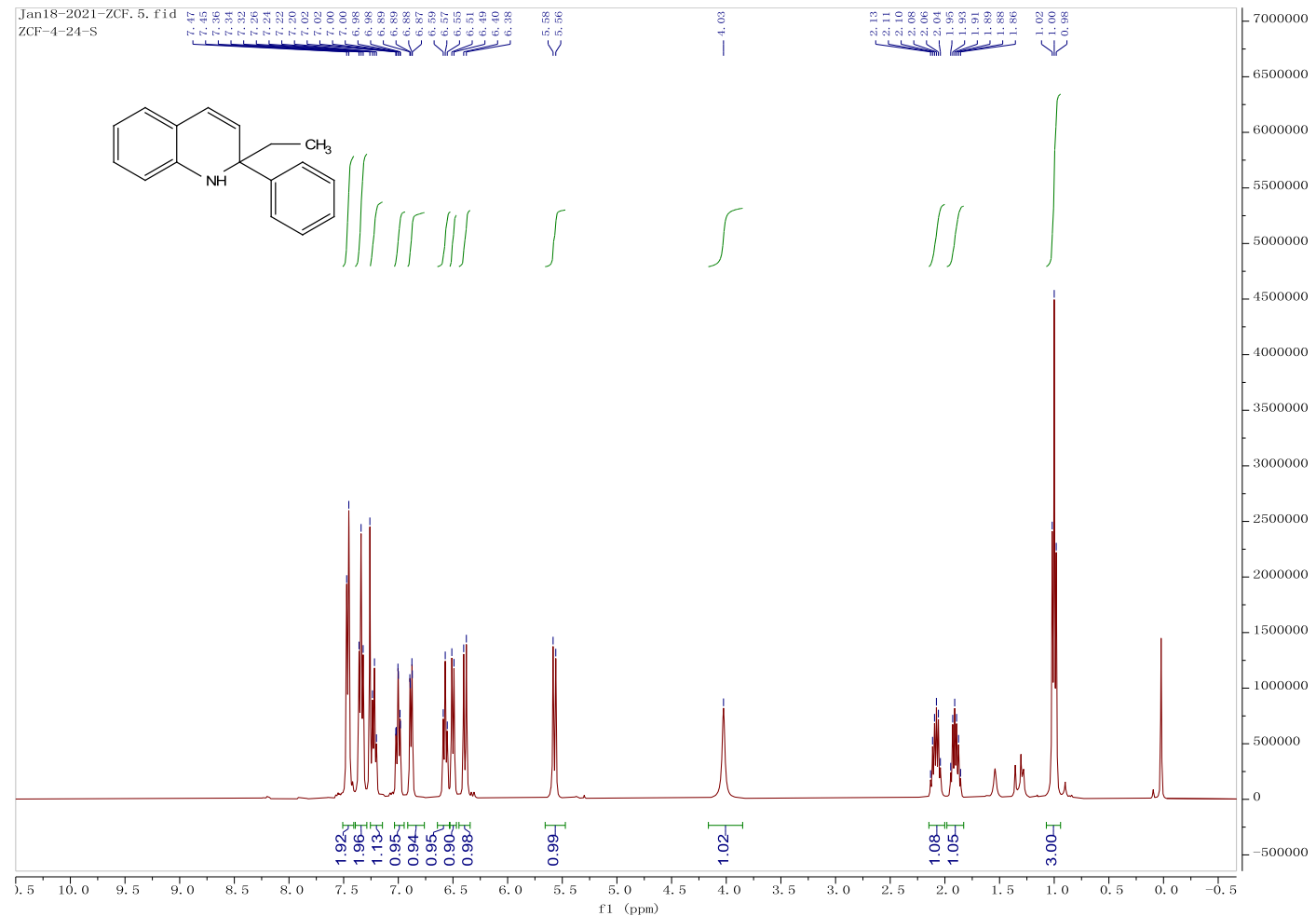

${ }^{1} \mathrm{H}$ NMR(400 MHz, Chloroform- $d$ )

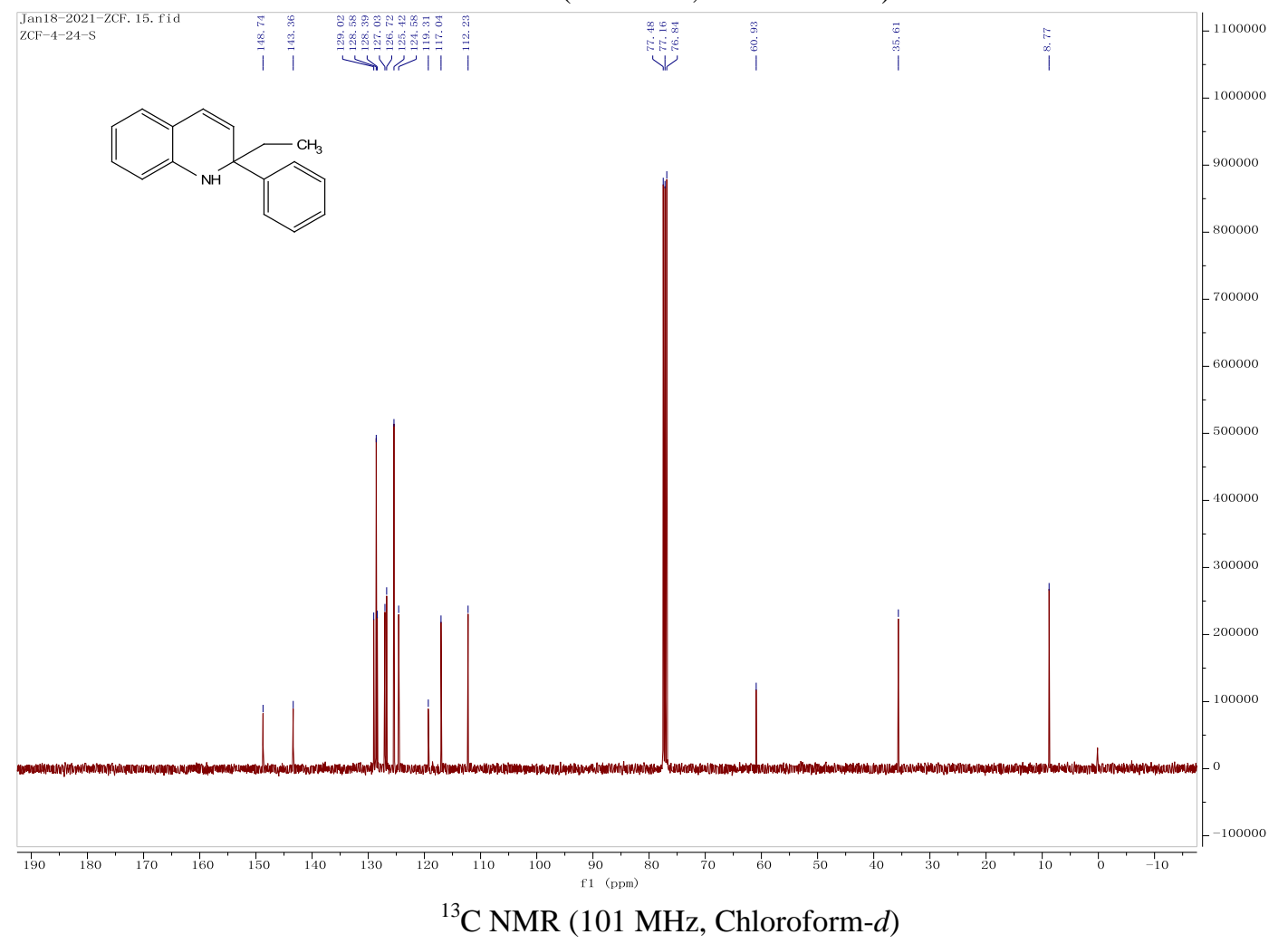


(S)-6-bromo-2-ethyl-2-phenyl-1,2-dihydroquinoline ((S)-2l)<smiles>CC[C@@]1(c2ccccc2)C=Cc2cc(Br)ccc2N1</smiles>

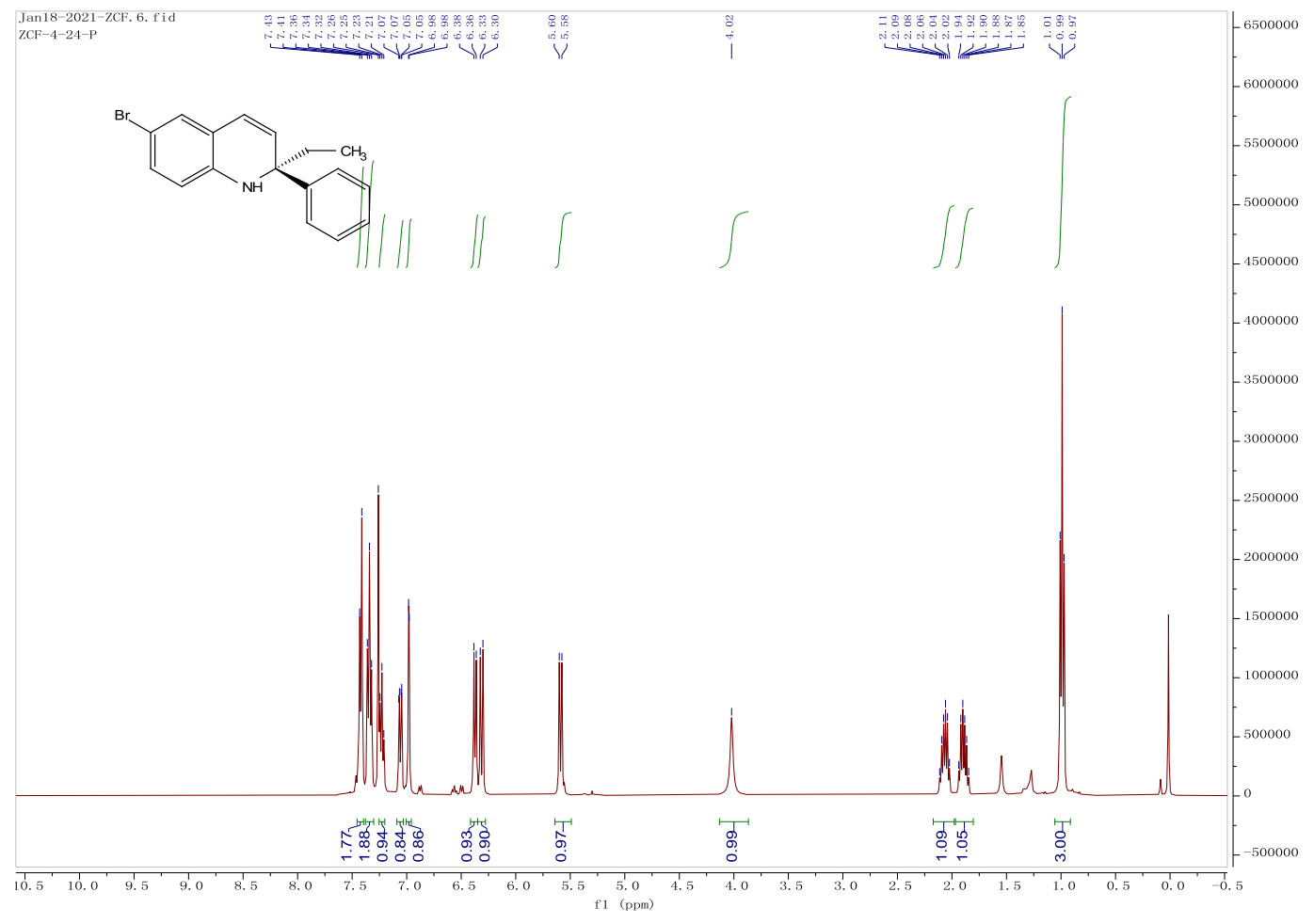

${ }^{1} \mathrm{H}$ NMR(400 MHz, Chloroform- $d$ )

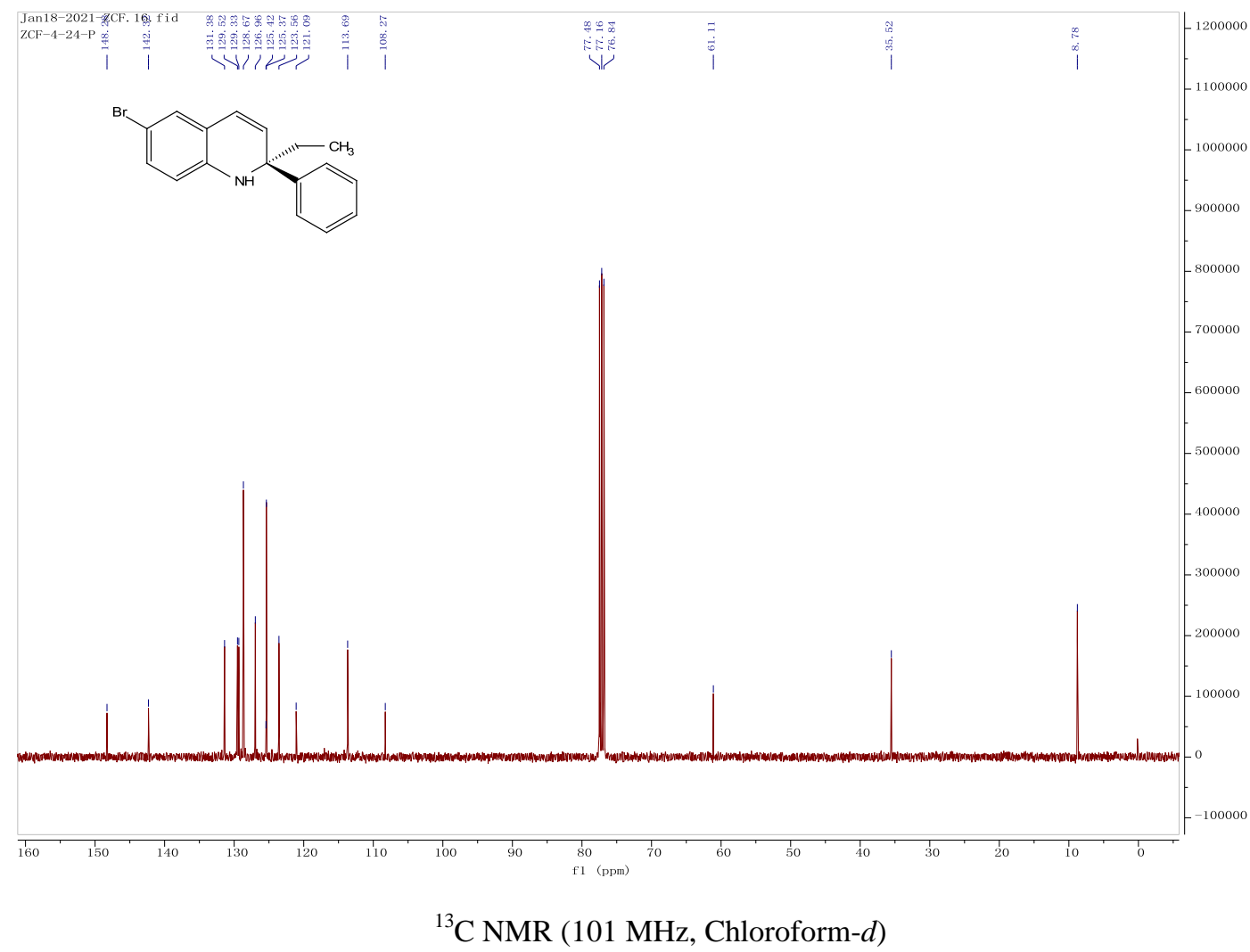


(R)-2-ethyl-2-(4-methoxyphenyl)-1,2-dihydroquinoline ((R)-1 m)

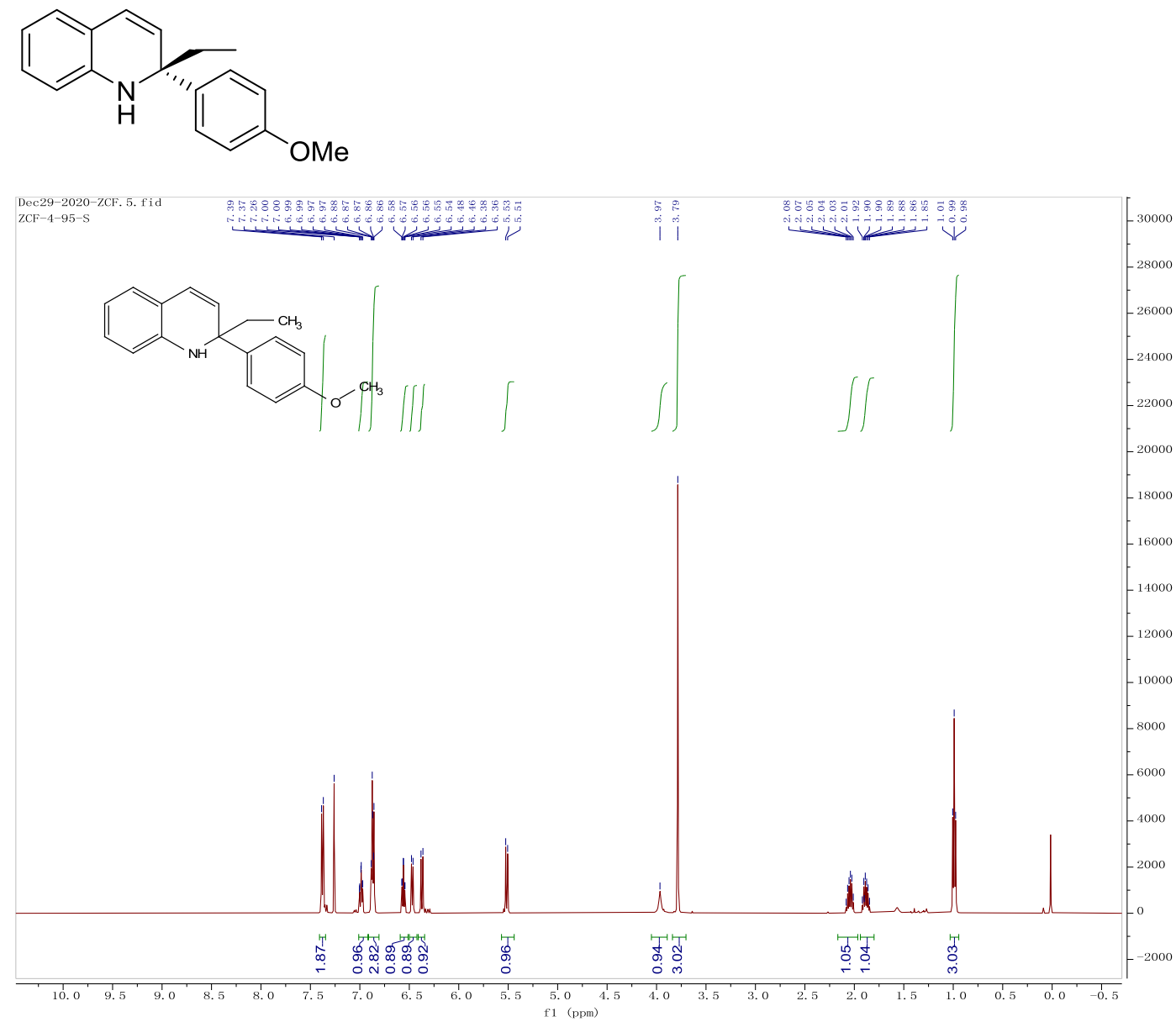

${ }^{1} \mathrm{H}$ NMR(500 MHz, Chloroform- $d$ )

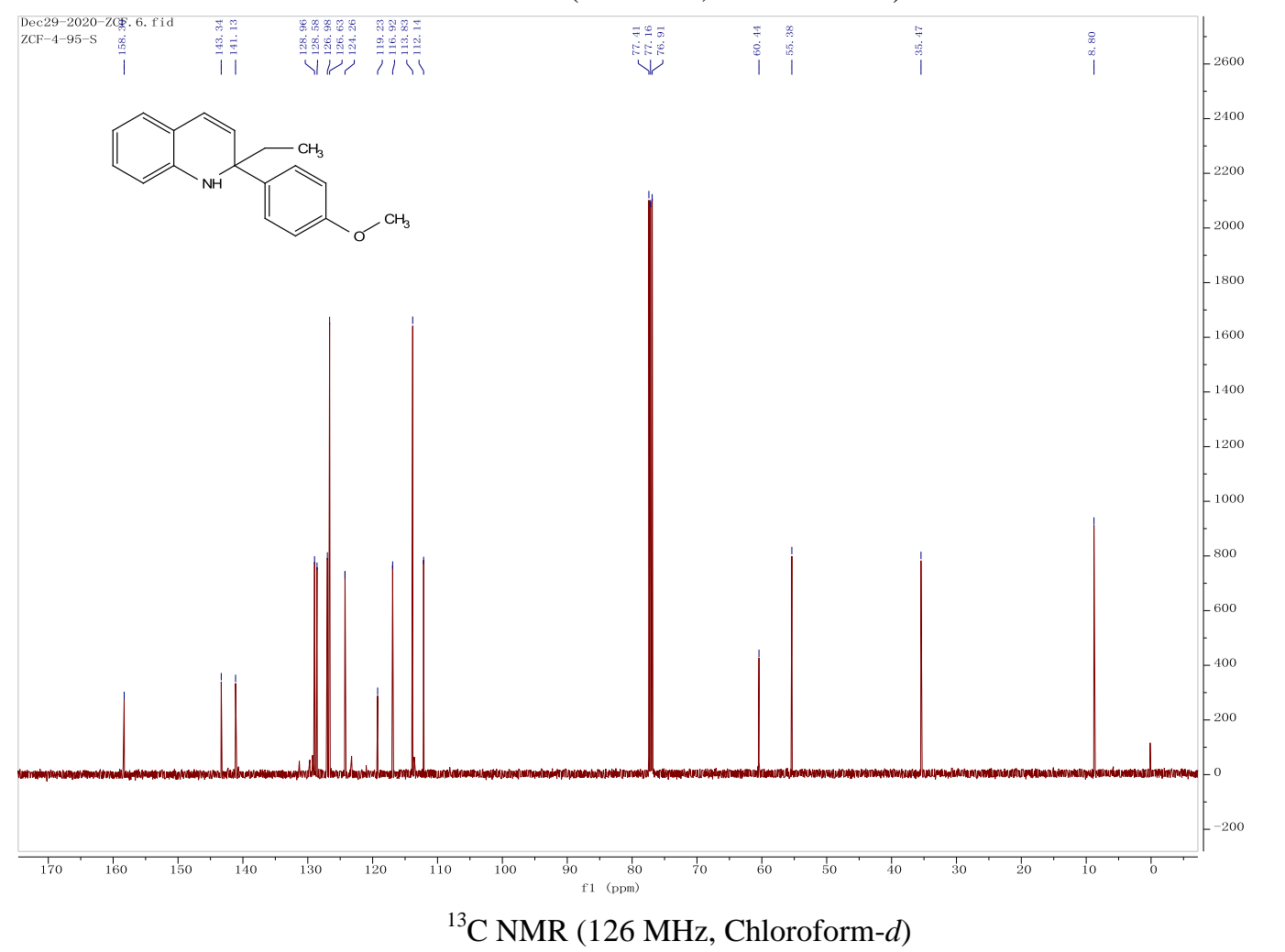


(S)-6-bromo-2-ethyl-2-(4-methoxyphenyl)-1,2-dihydroquinoline ((S)-2m)

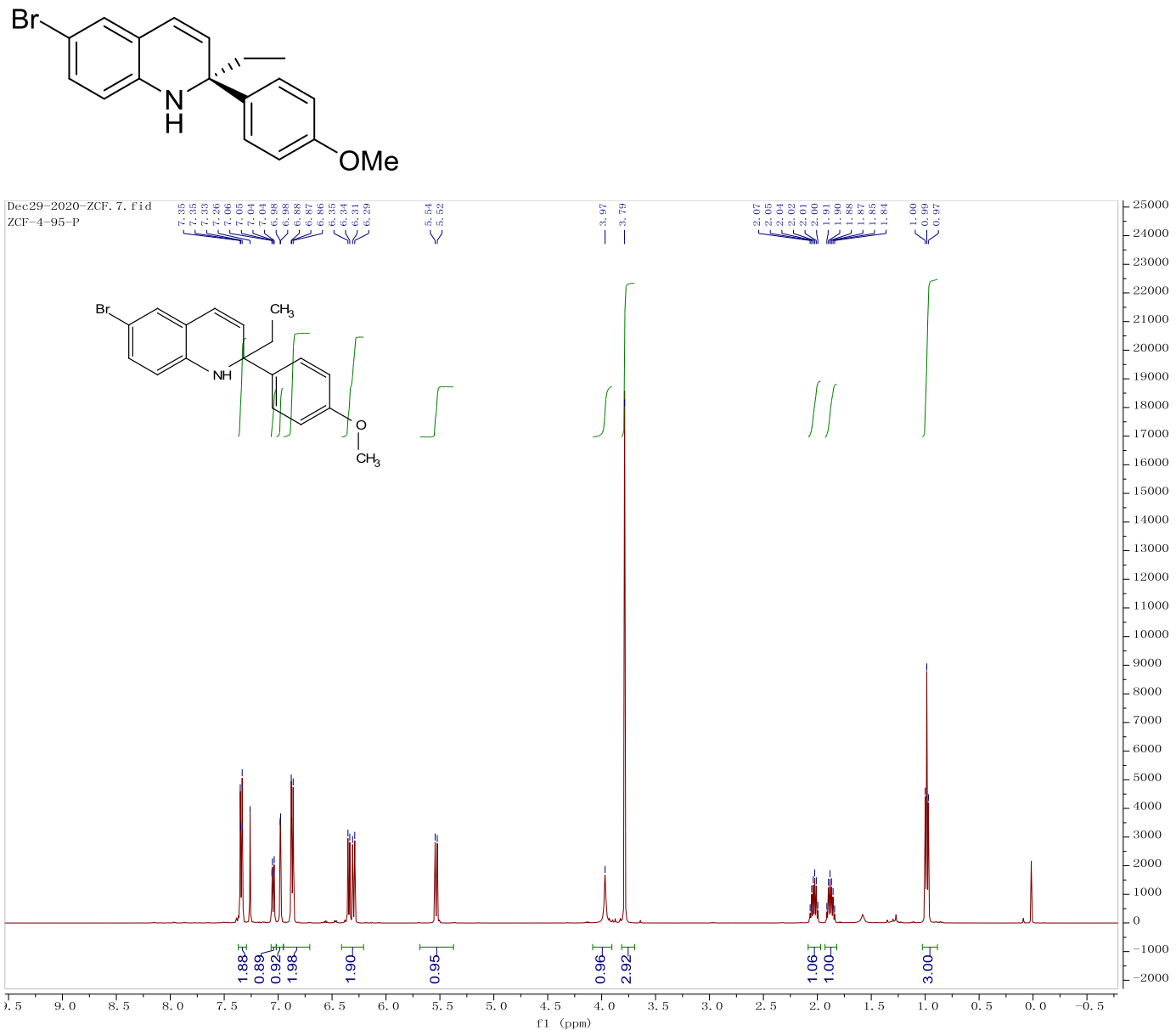

${ }^{1} \mathrm{H}$ NMR $(500 \mathrm{MHz}$, Chloroform- $d$ )

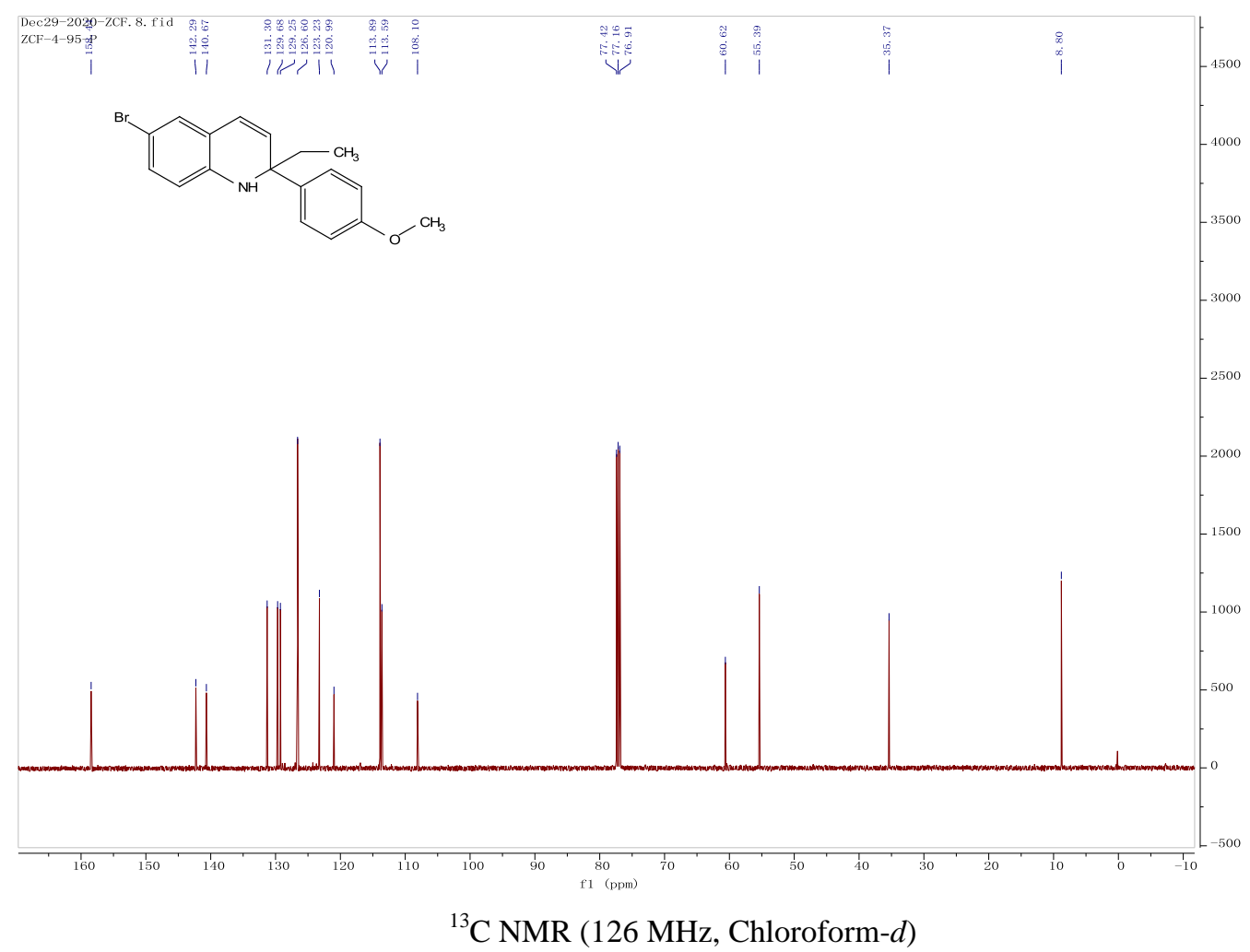


(R)-2-ethyl-2-(3-methoxyphenyl)-1,2-dihydroquinoline ((R)-1n)<smiles>CCC1(c2cccc(OC)c2)C=Cc2ccccc2N1</smiles>

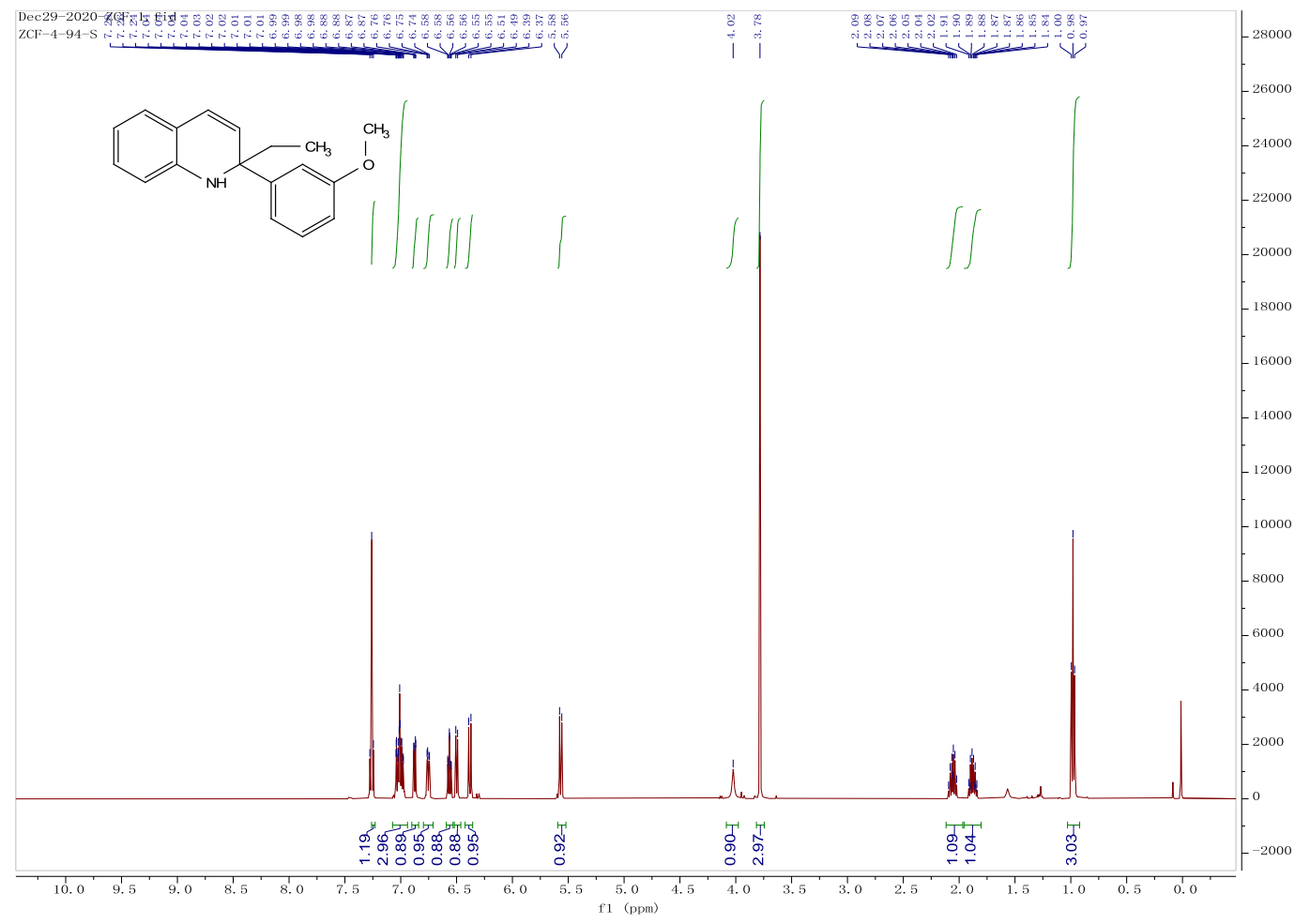

${ }^{1} \mathrm{H}$ NMR(500 MHz, Chloroform- $d$ )

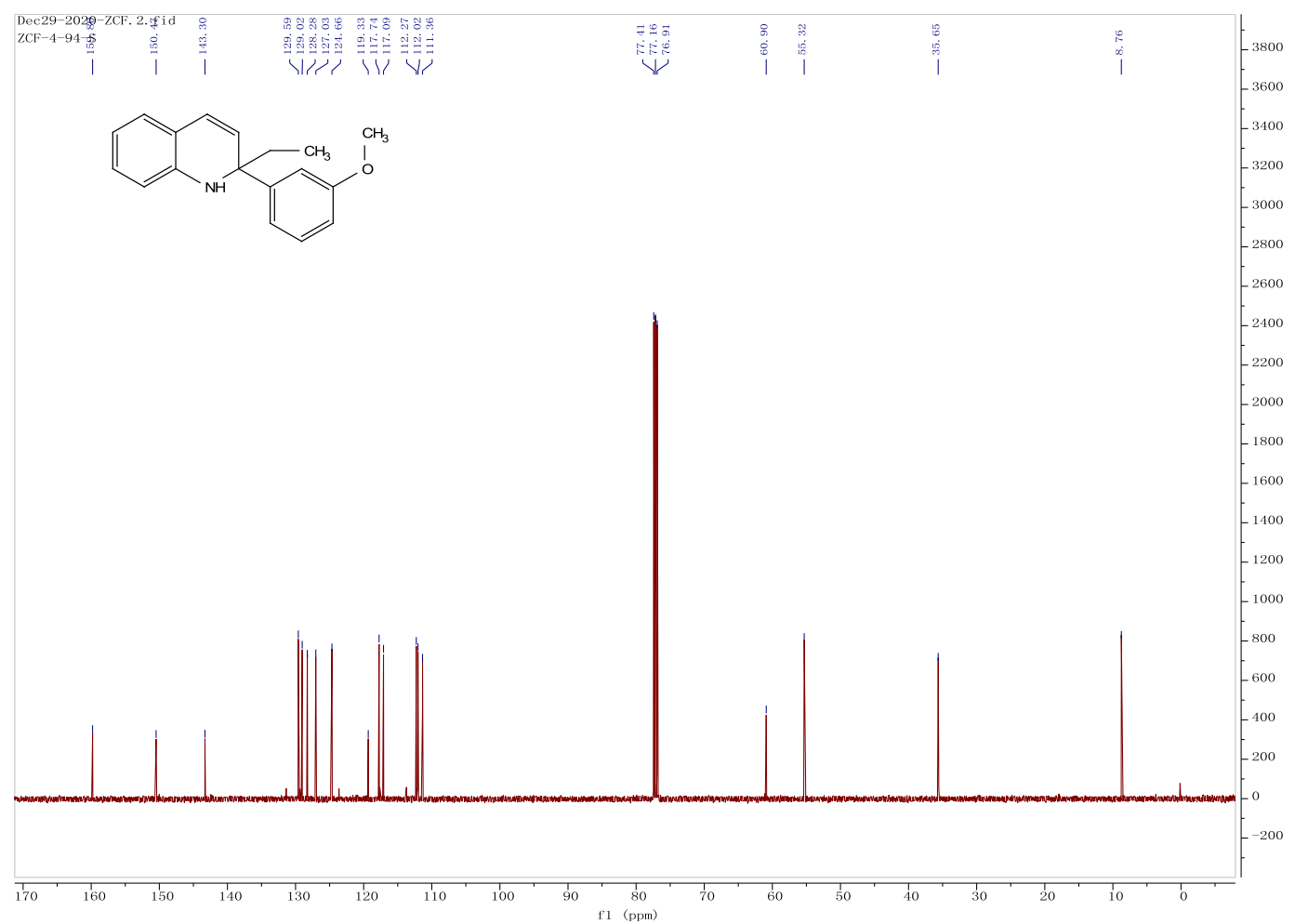

${ }^{13} \mathrm{C}$ NMR (126 MHz, Chloroform- $d$ ) 
(S)-6-bromo-2-ethyl-2-(3-methoxyphenyl)-1,2-dihydroquinoline ((S)-2n)<smiles>CC[C@](C)(c1cccc(OC)c1)C1C=Cc2cc(Br)ccc2N1</smiles>

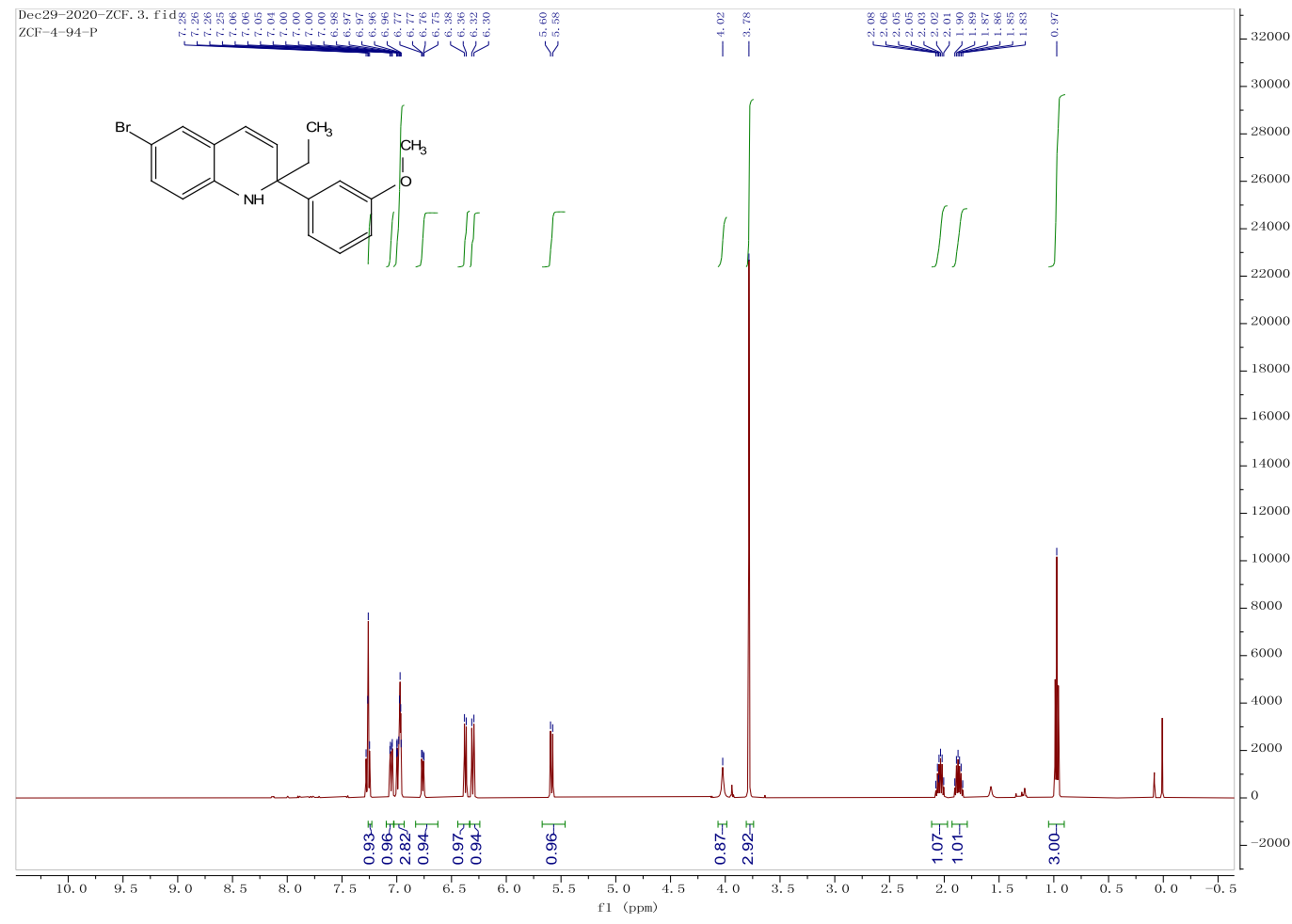

${ }^{1} \mathrm{H}$ NMR(500 MHz, Chloroform- $d$ )

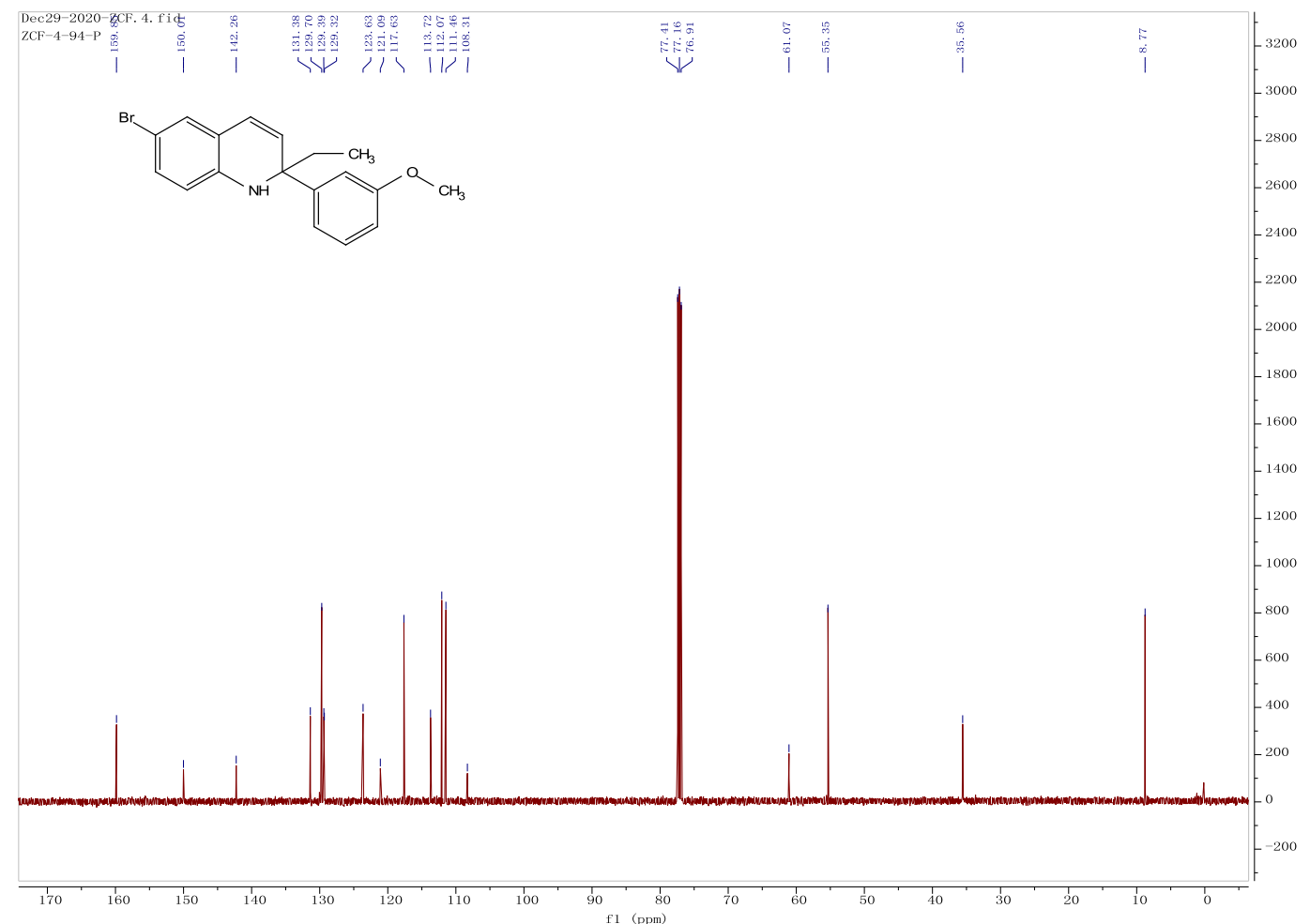

${ }^{13} \mathrm{C}$ NMR (126 MHz, Chloroform- $d$ ) 
(R)-2-methyl-2-phenyl-1,2,3,4-tetrahydroquinoline $((R)$-1o)<smiles>C[C@]1(c2ccccc2)CCc2ccccc2N1</smiles>

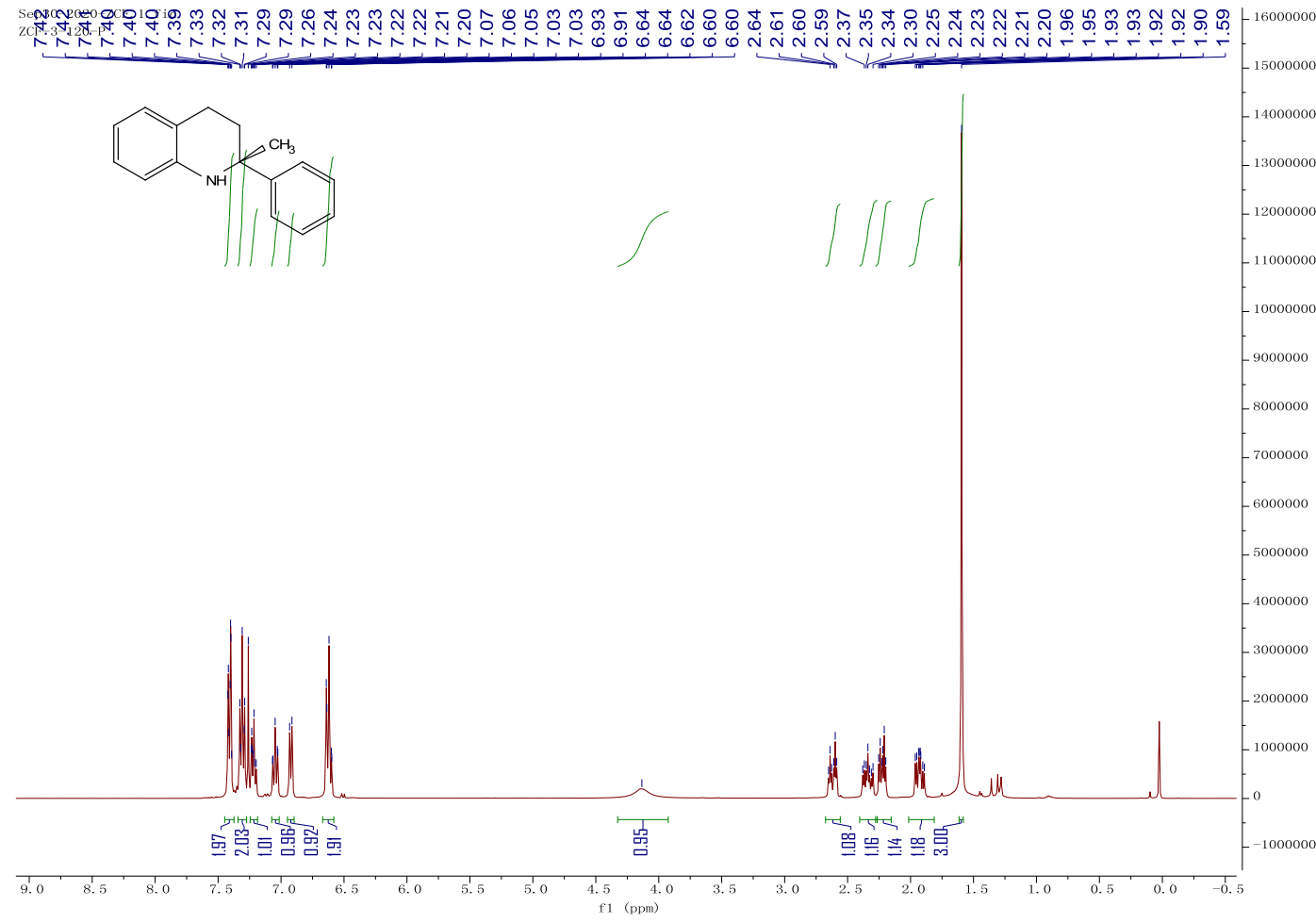

${ }^{1} \mathrm{H}$ NMR(400 MHz, Chloroform- $d$ )

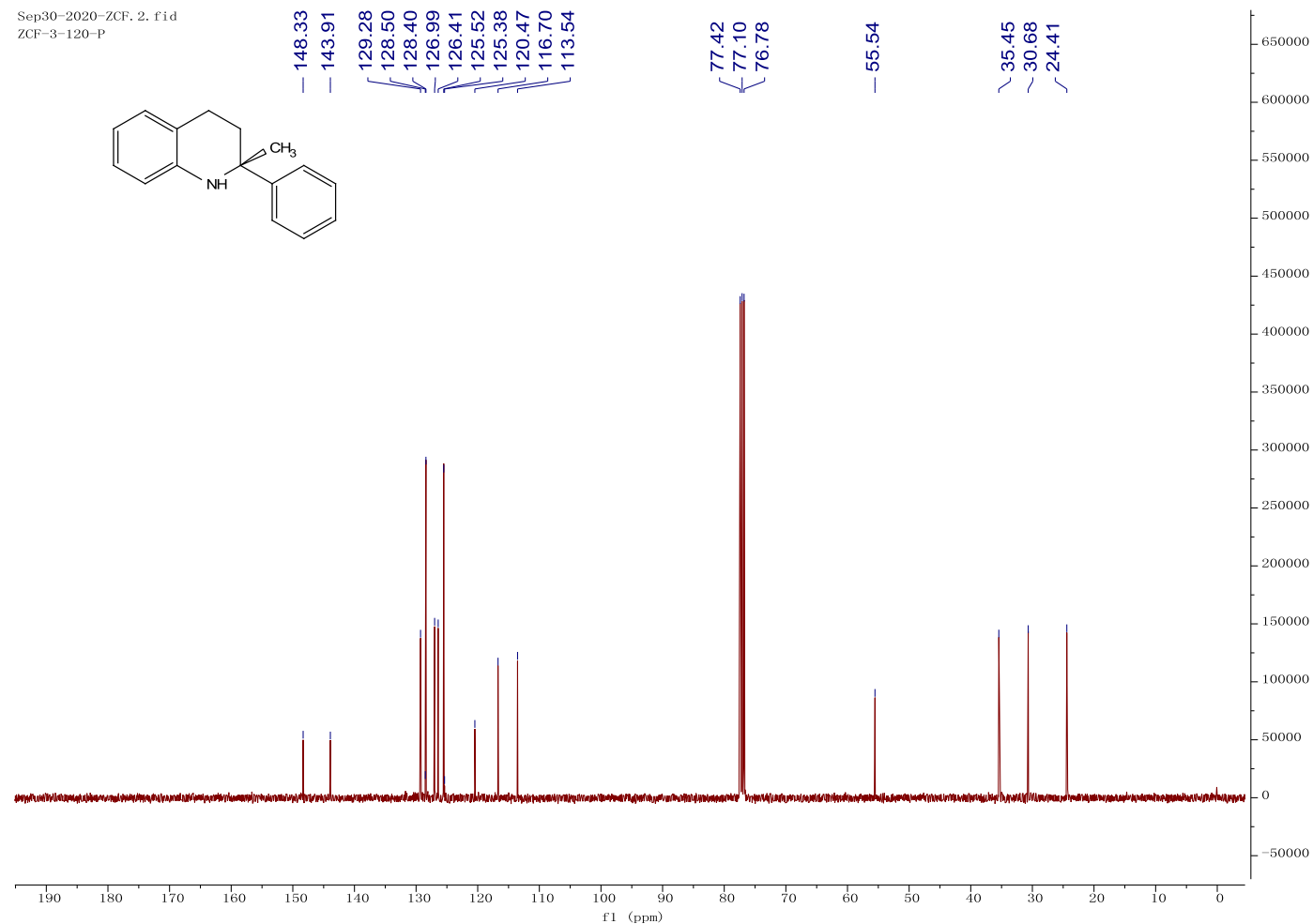

${ }^{13} \mathrm{C}$ NMR (101 MHz, Chloroform- $d$ ) 
(S)-6-bromo-2-methyl-2-phenyl-1,2,3,4-tetrahydroquinoline ((S)-2o)

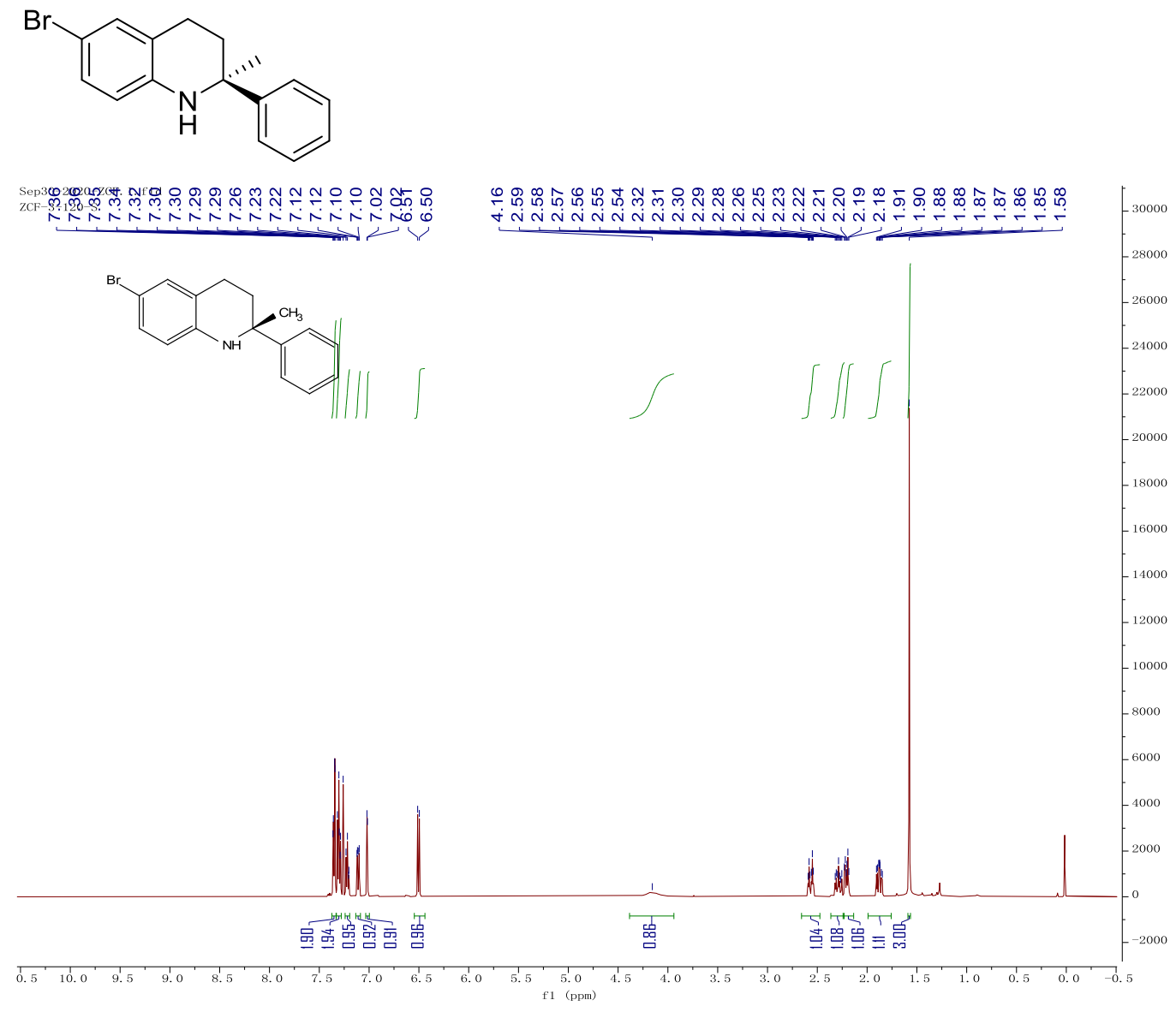

${ }^{1} \mathrm{H}$ NMR(500 MHz, Chloroform- $d$ )

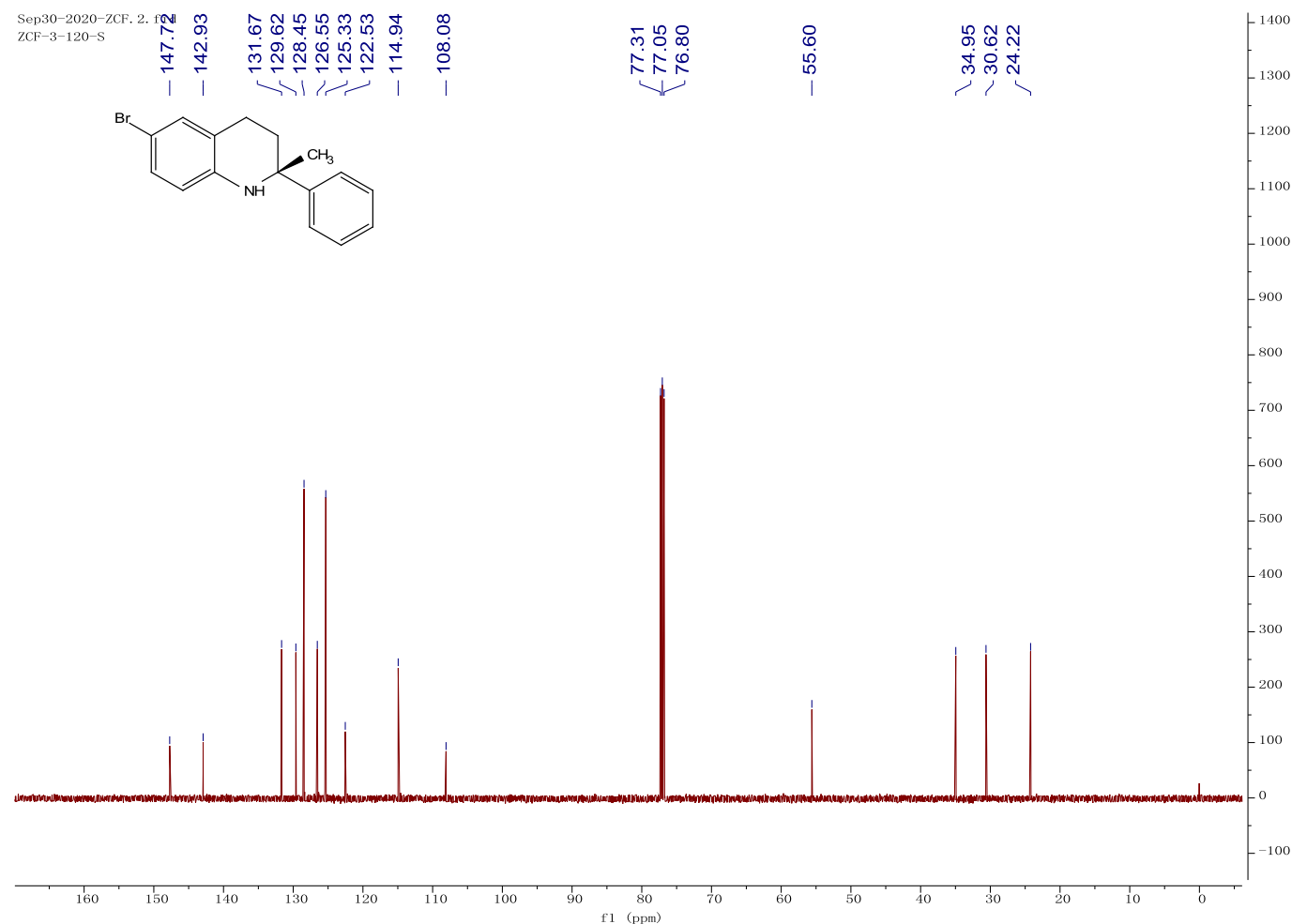

${ }^{13} \mathrm{C}$ NMR (126 MHz, Chloroform- $d$ ) 
(S)-6-iodo-2-methyl-2-pheyl-1,2-dihydroquinoline ((S)-3a)

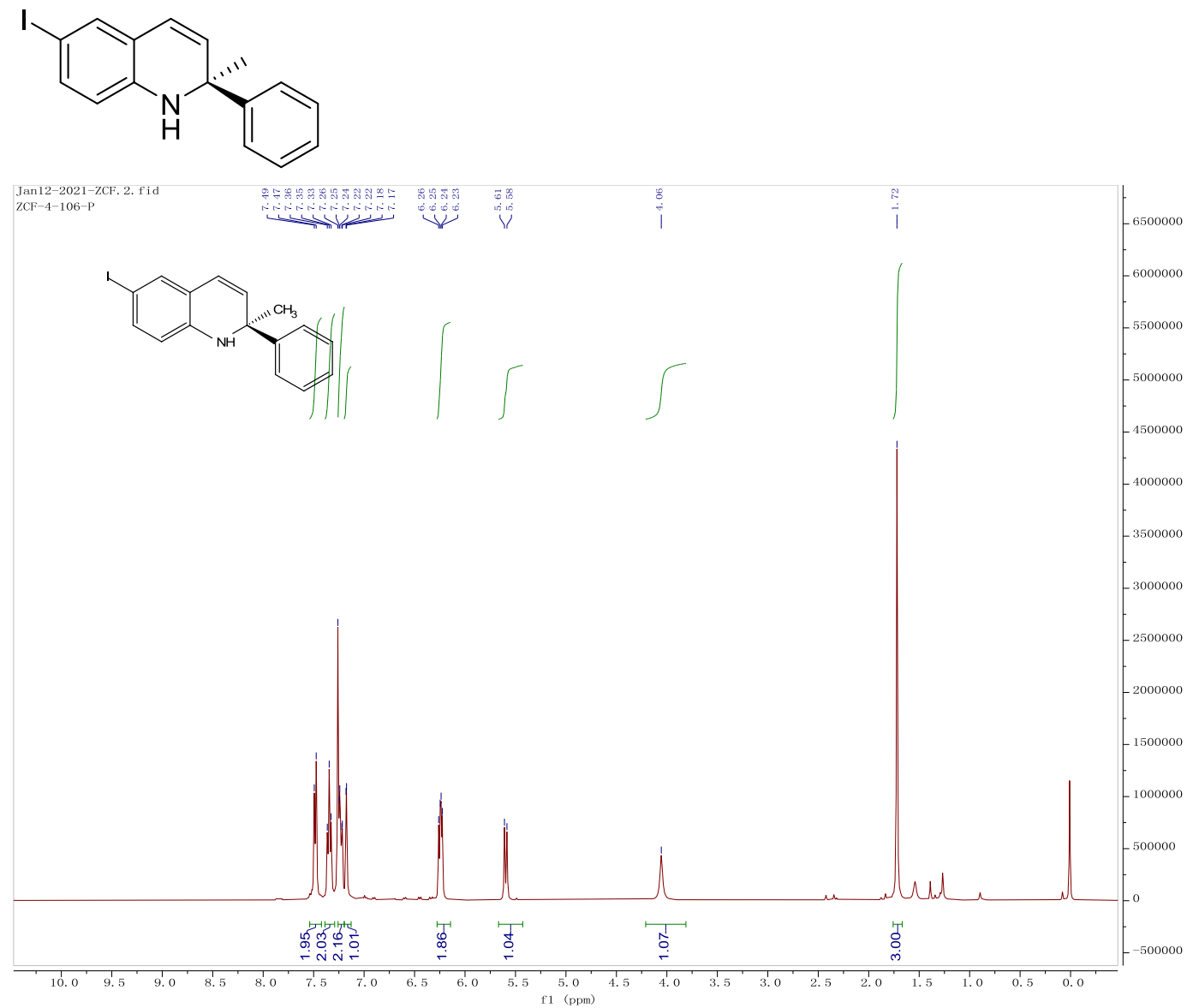

${ }^{1} \mathrm{H}$ NMR(400 MHz, Chloroform- $d$ )

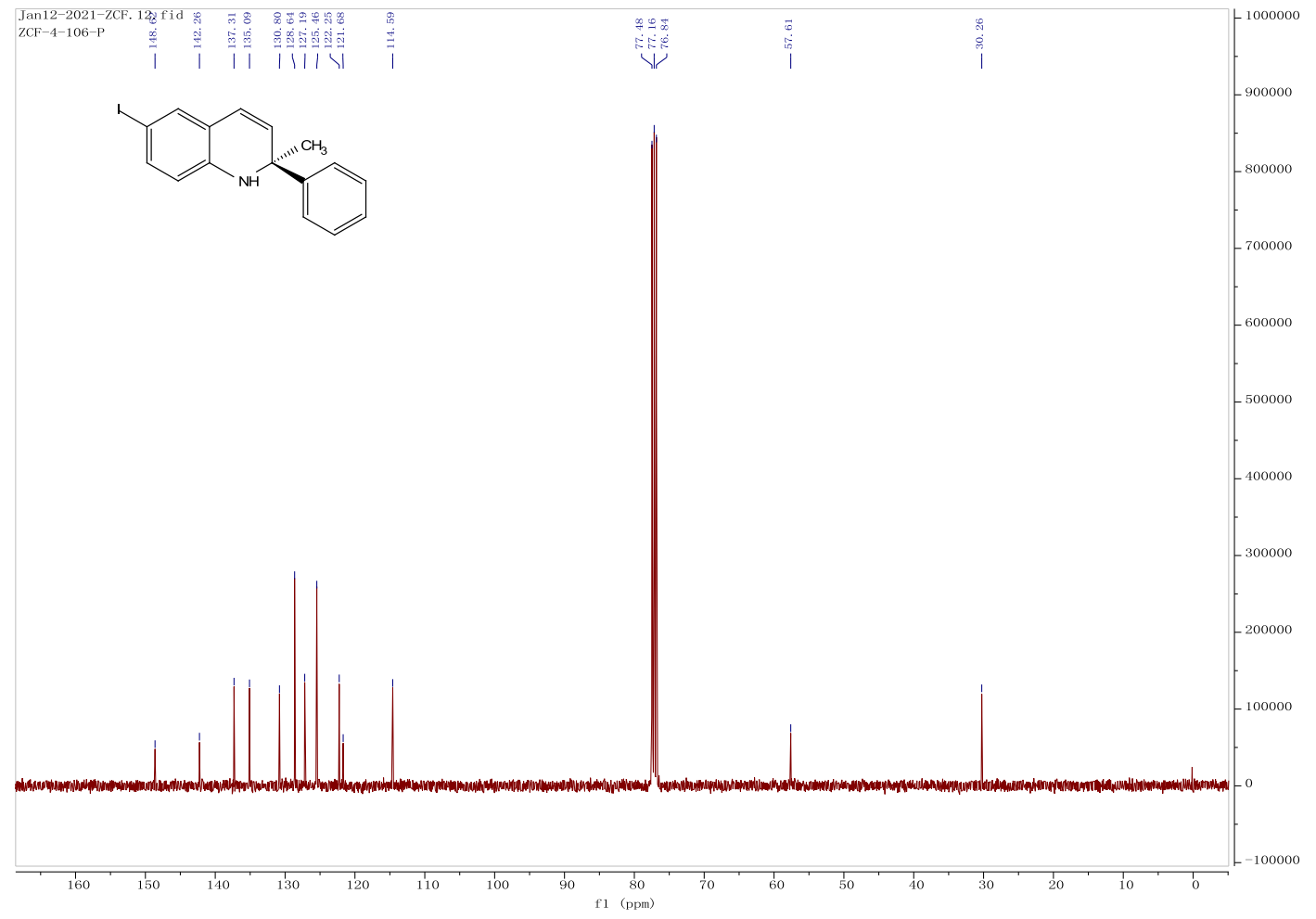

${ }^{13} \mathrm{C}$ NMR (101 MHz, Chloroform- $d$ ) 
(S)-2-methyl-2-phenyl-6-(4,4,5,5-tetramethyl-1,3,2-dioxaborolan-2-yl)-1,2-dihydroquinoline $((S)-\mathbf{4 a})$<smiles>CC1(C)OB(c2ccc3c(c2)C=C[C@](C)(c2ccccc2)N3)OC1(C)C</smiles>

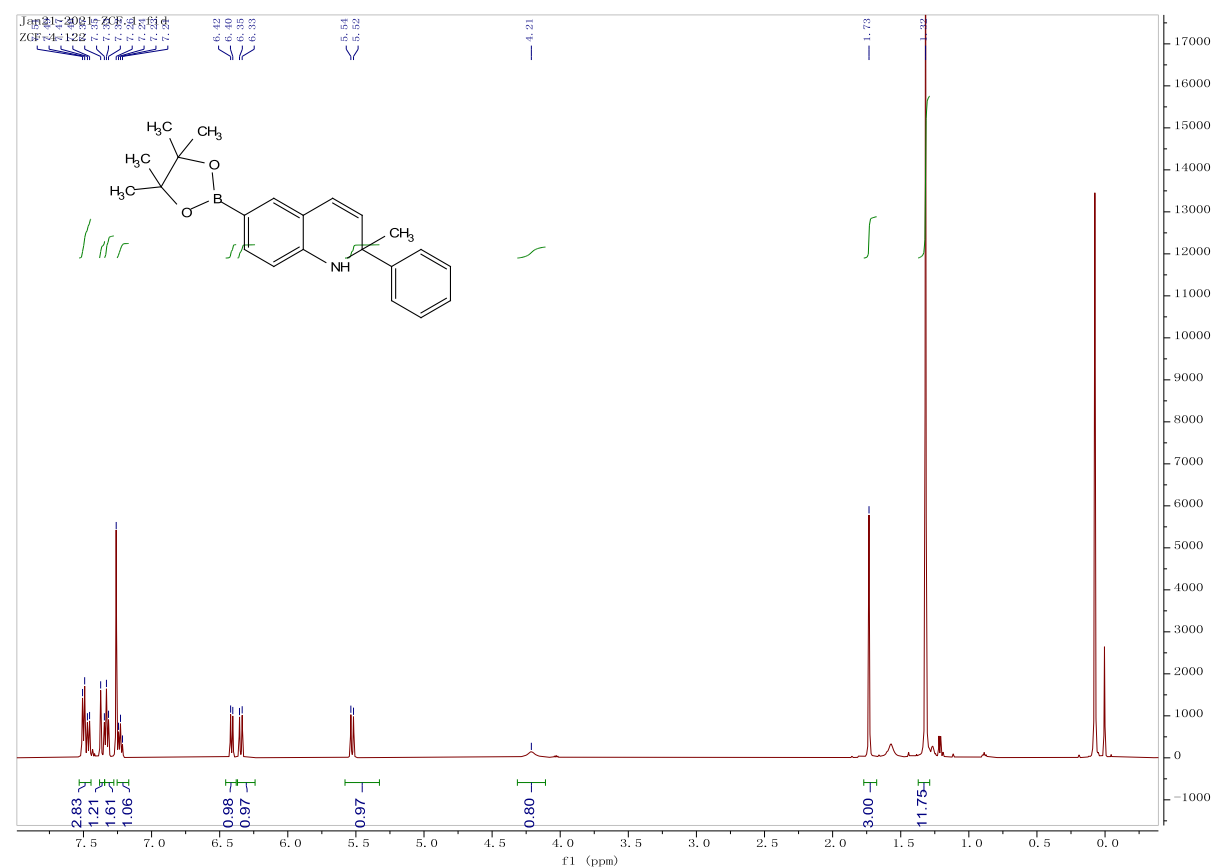

${ }^{1} \mathrm{H}$ NMR(500 MHz, Chloroform- $d$ )

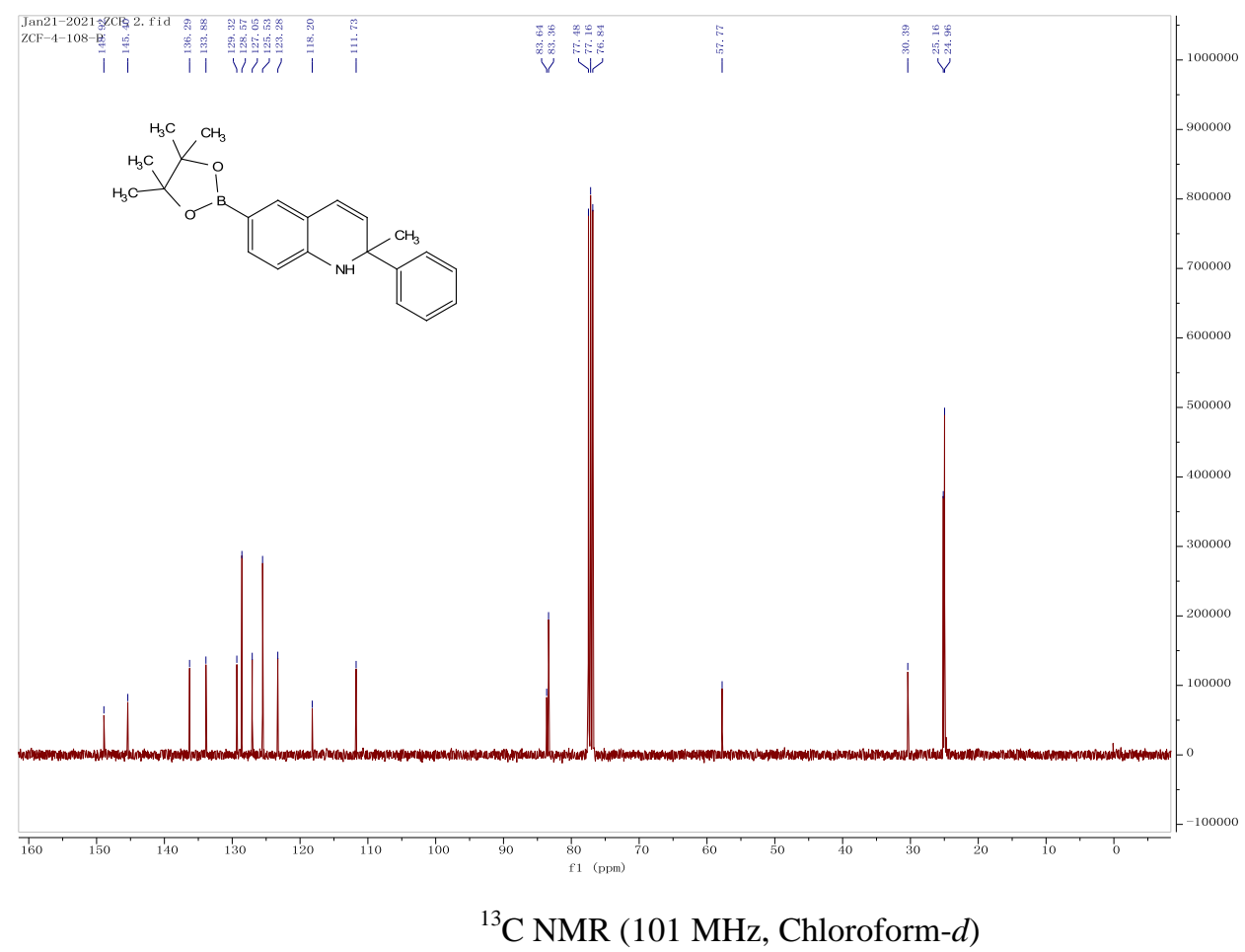


(R)-2,3-dimethyl-2-phenyl-1,2-dihydroquinoline $((R)-1 \mathbf{p})$<smiles>CC1=Cc2ccccc2N[C@@]1(C)c1ccccc1</smiles>

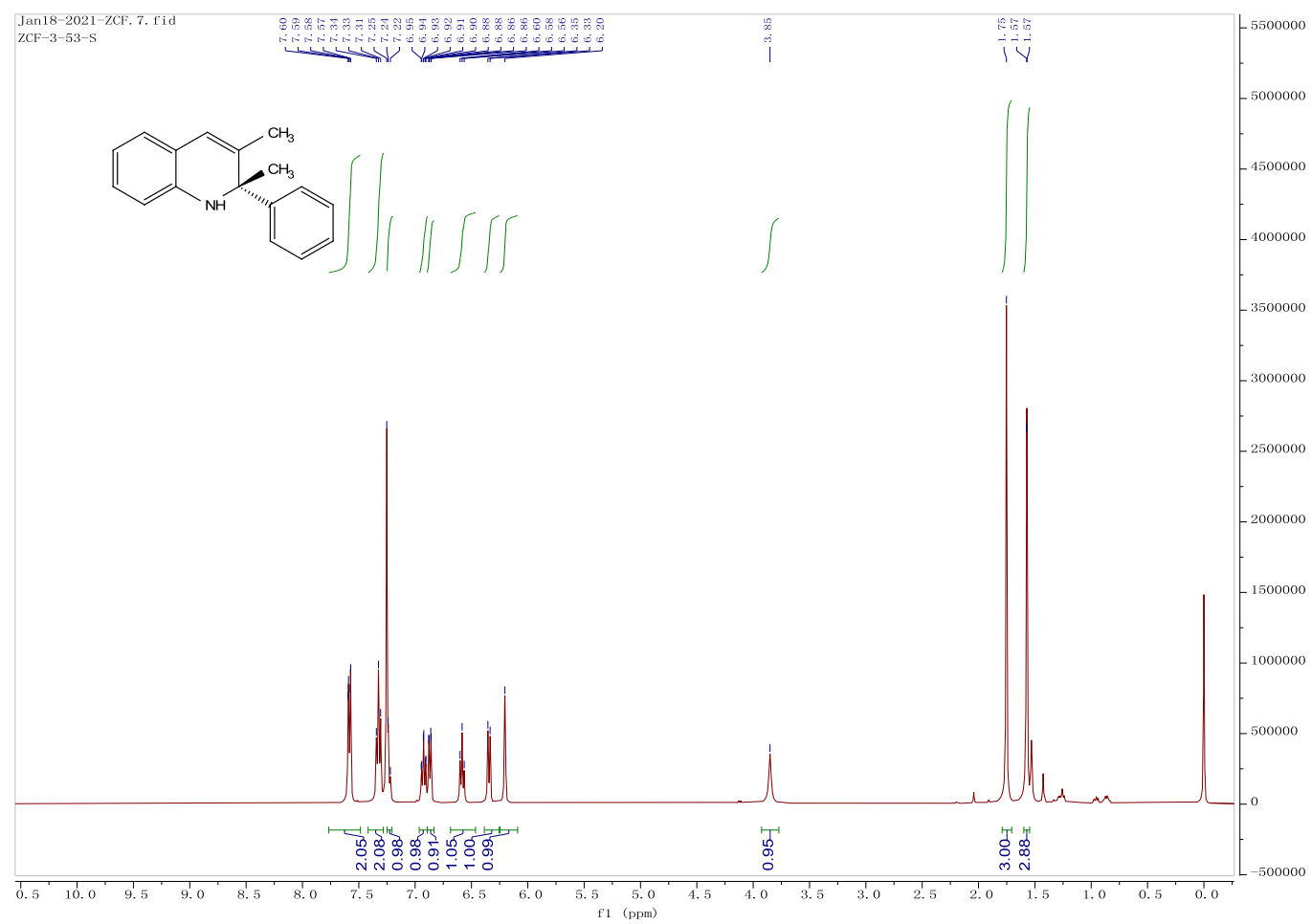

${ }^{1} \mathrm{H}$ NMR(400 MHz, Chloroform- $d$ )

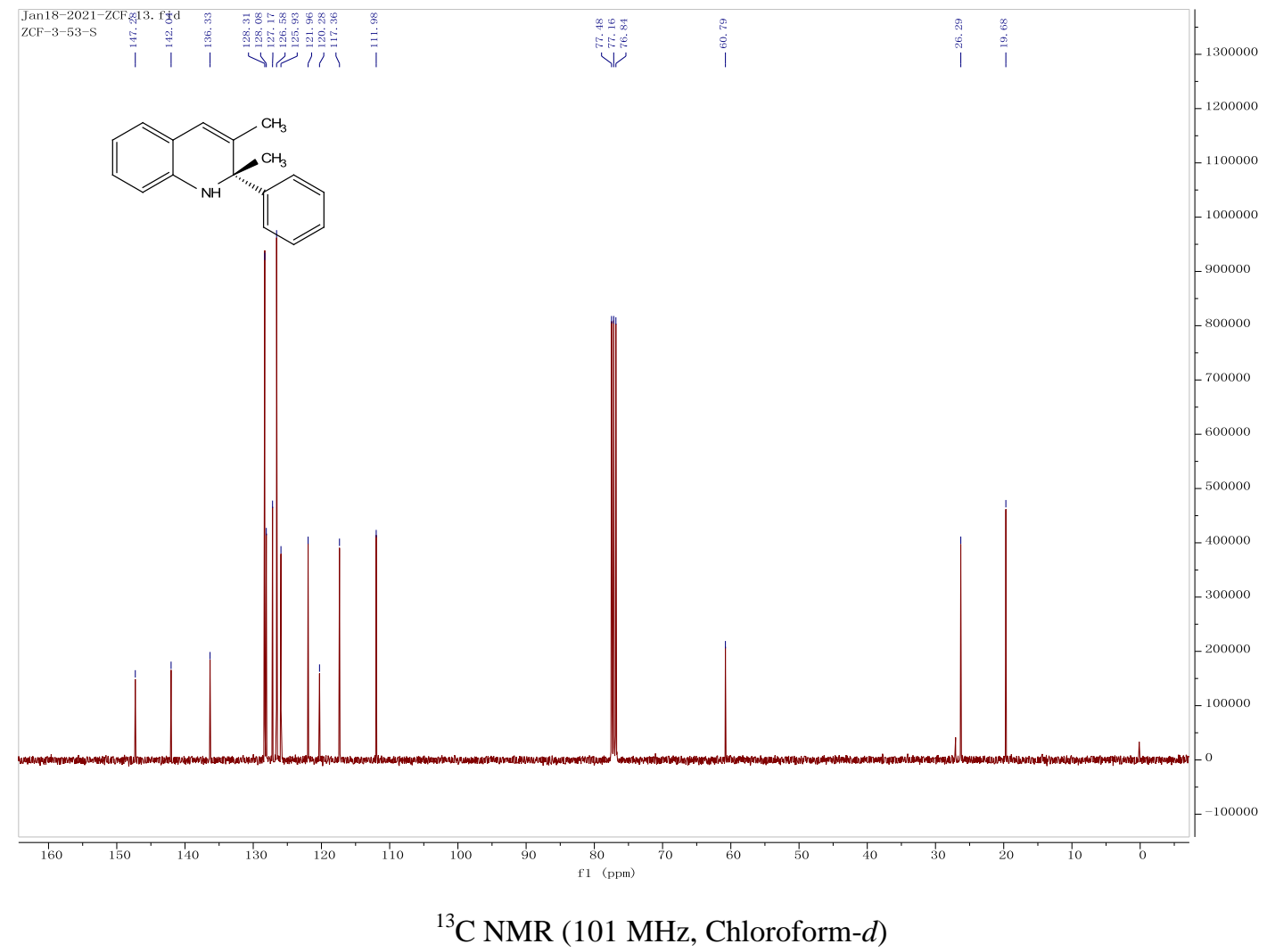


(S)-2,3-dimethyl-2-phenyl-6-(4,4,5,5-tetramethyl-1,3,2-dioxaborolan-2-yl)-1,2-dihydroquinol -ine $((S)-\mathbf{4 p})$
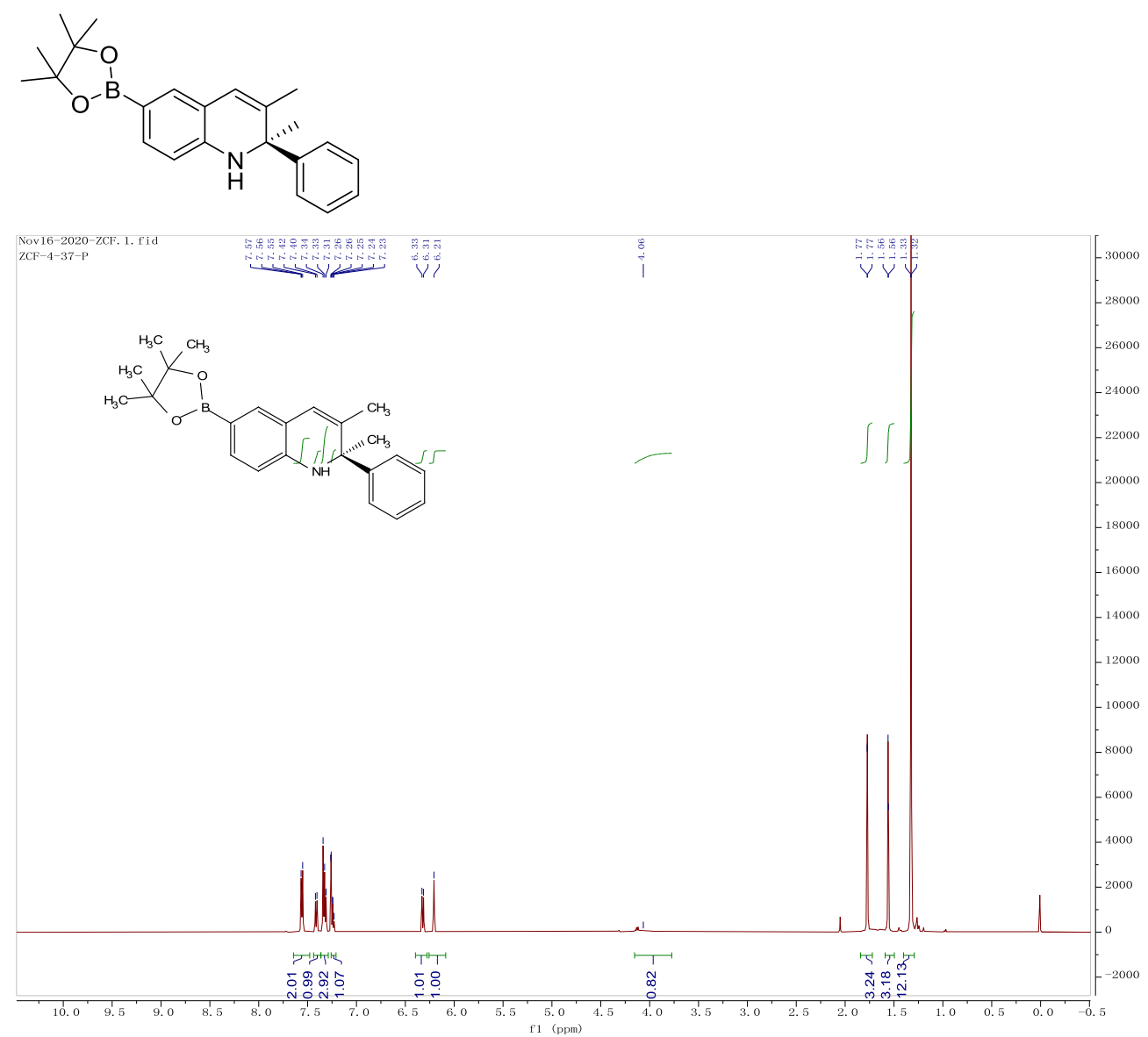

${ }^{1} \mathrm{H} \mathrm{NMR}(500 \mathrm{MHz}$, Chloroform- $d)$

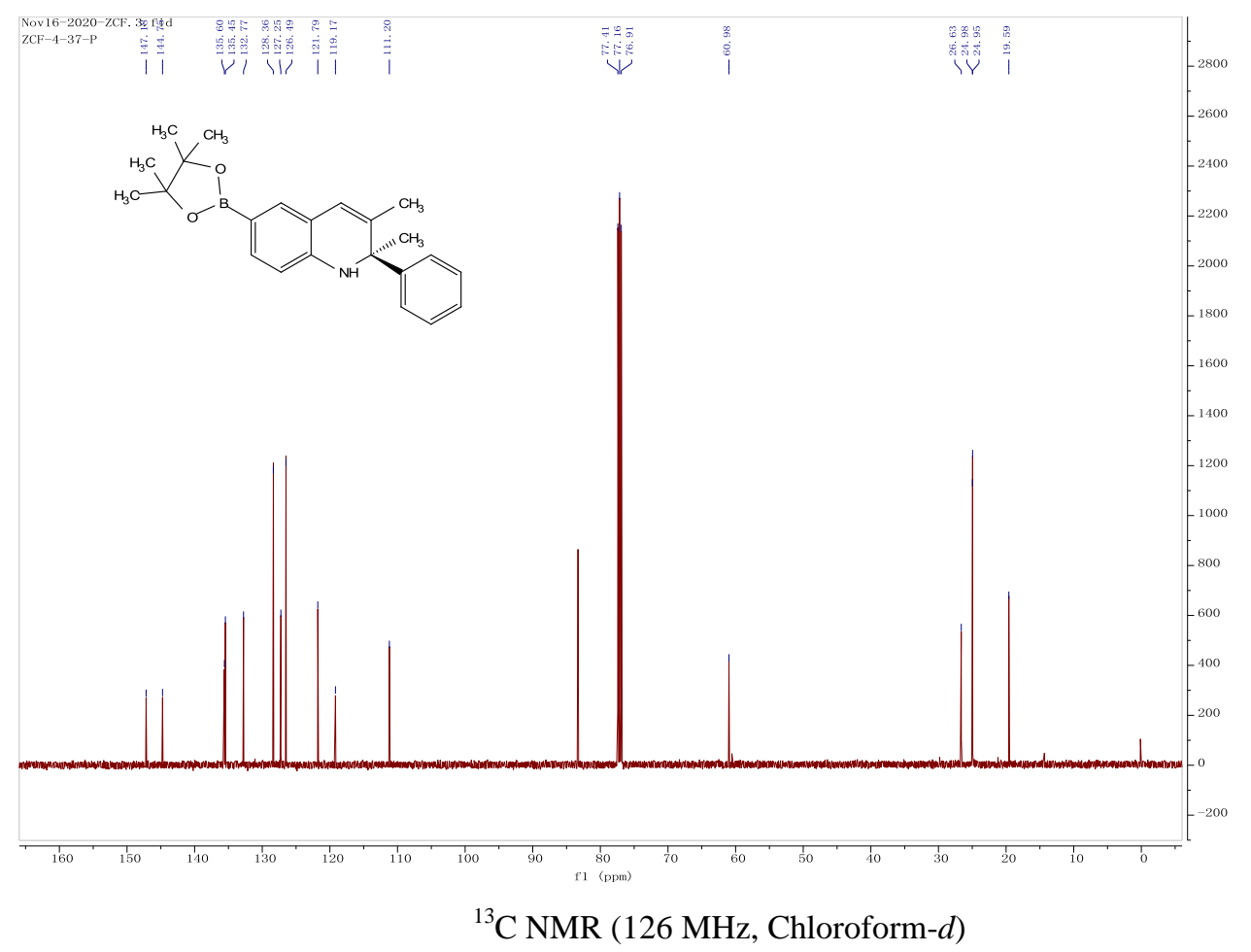


(R)-2-(3,5-dimethylphenyl)-2-methyl-1,2-dihydroquinoline ((R)-1q)<smiles>Cc1cc(C)cc([C@]2(C)C=Cc3ccccc3N2)c1</smiles>

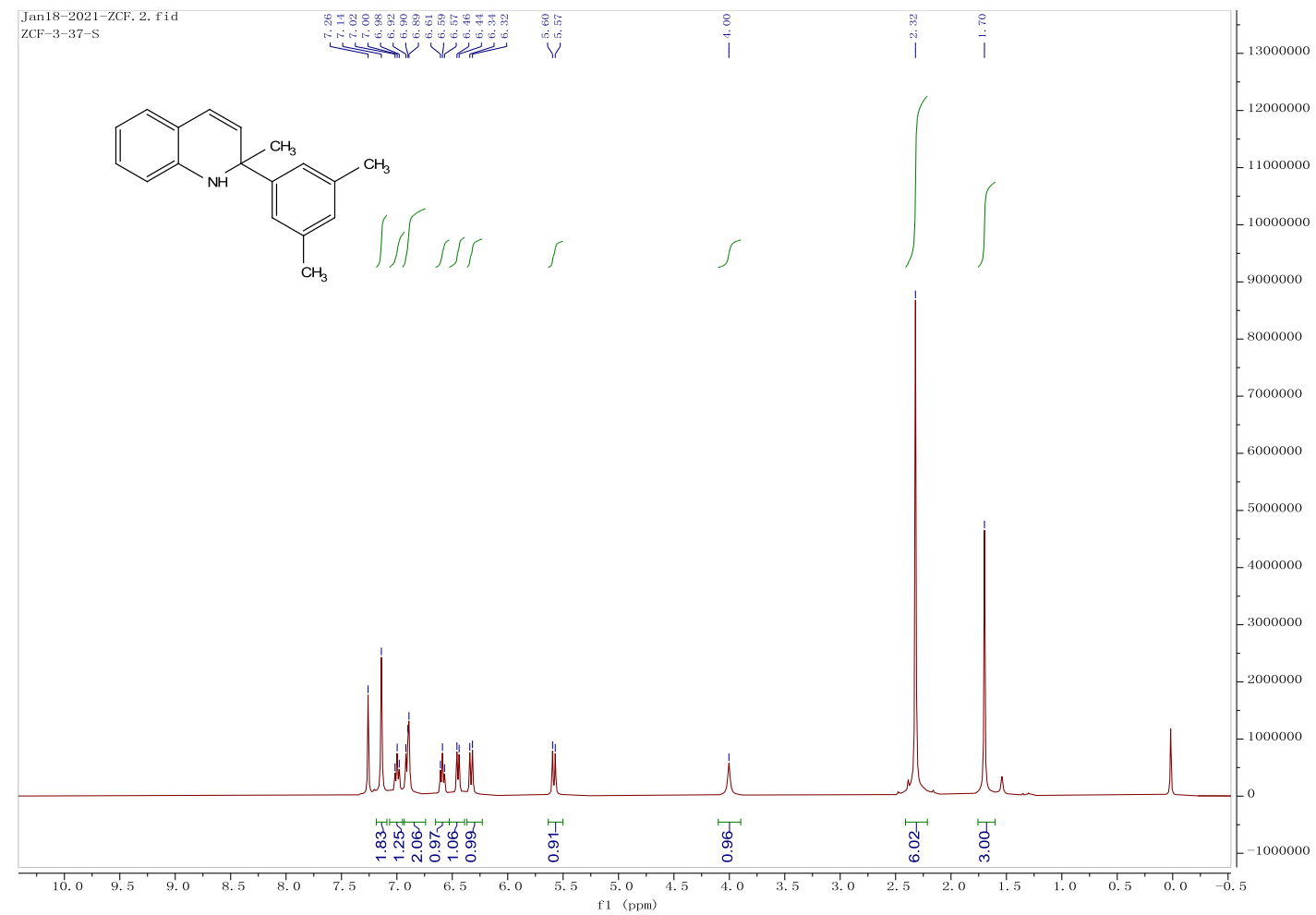

${ }^{1} \mathrm{H}$ NMR(400 MHz, Chloroform- $d$ )

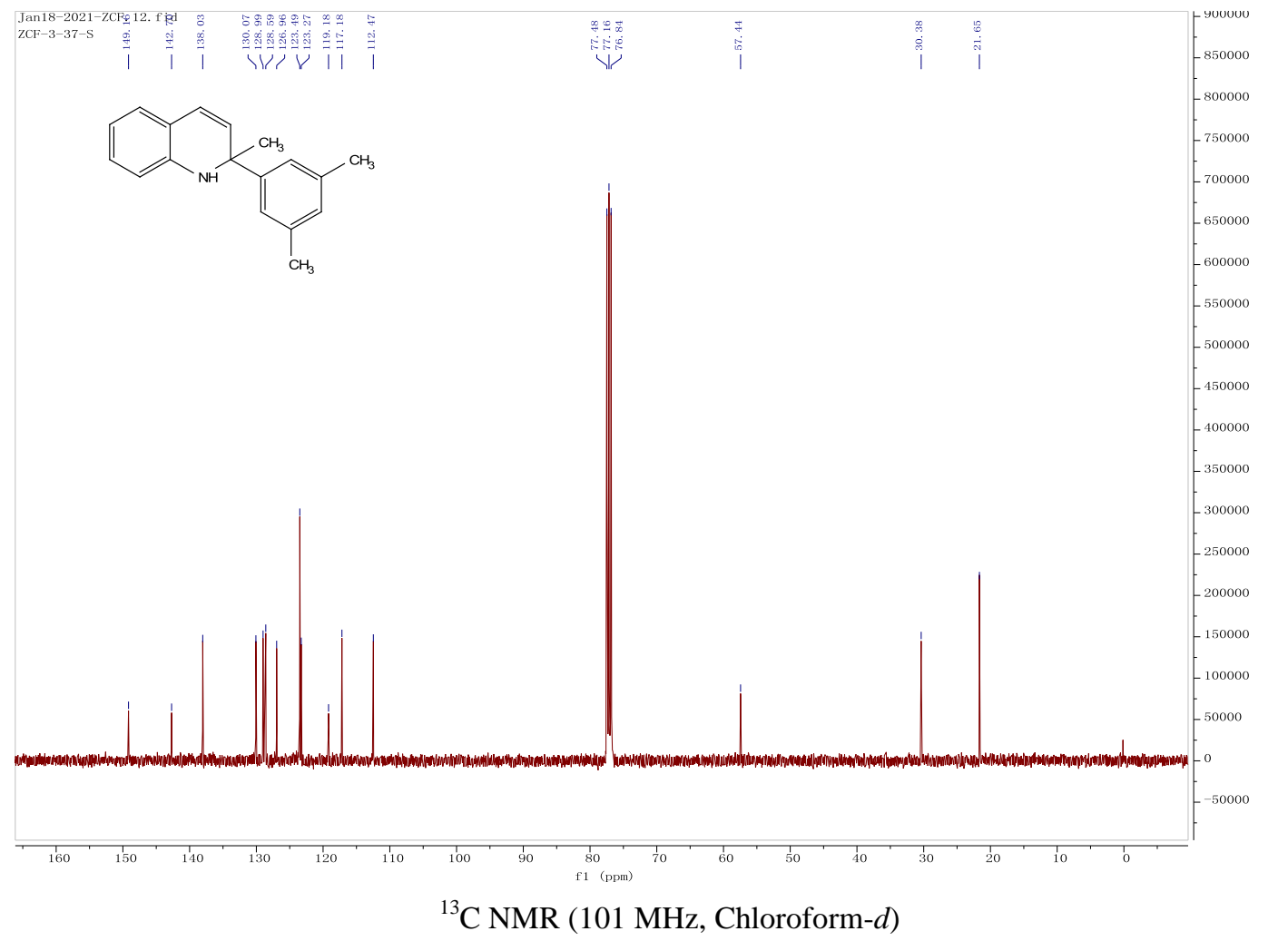


(S)-2-(3,5-dimethylphenyl)-2-methyl-6-(4,4,5,5-tetramethyl-1,3,2-dioxaborolan-2-yl)-1,2-dih ydroquinoline $((S)-\mathbf{4 q})$<smiles>Cc1cc(C)cc([C@]2(C)C=Cc3cc(B4OC(C)(C)C(C)(C)O4)ccc3N2)c1</smiles>

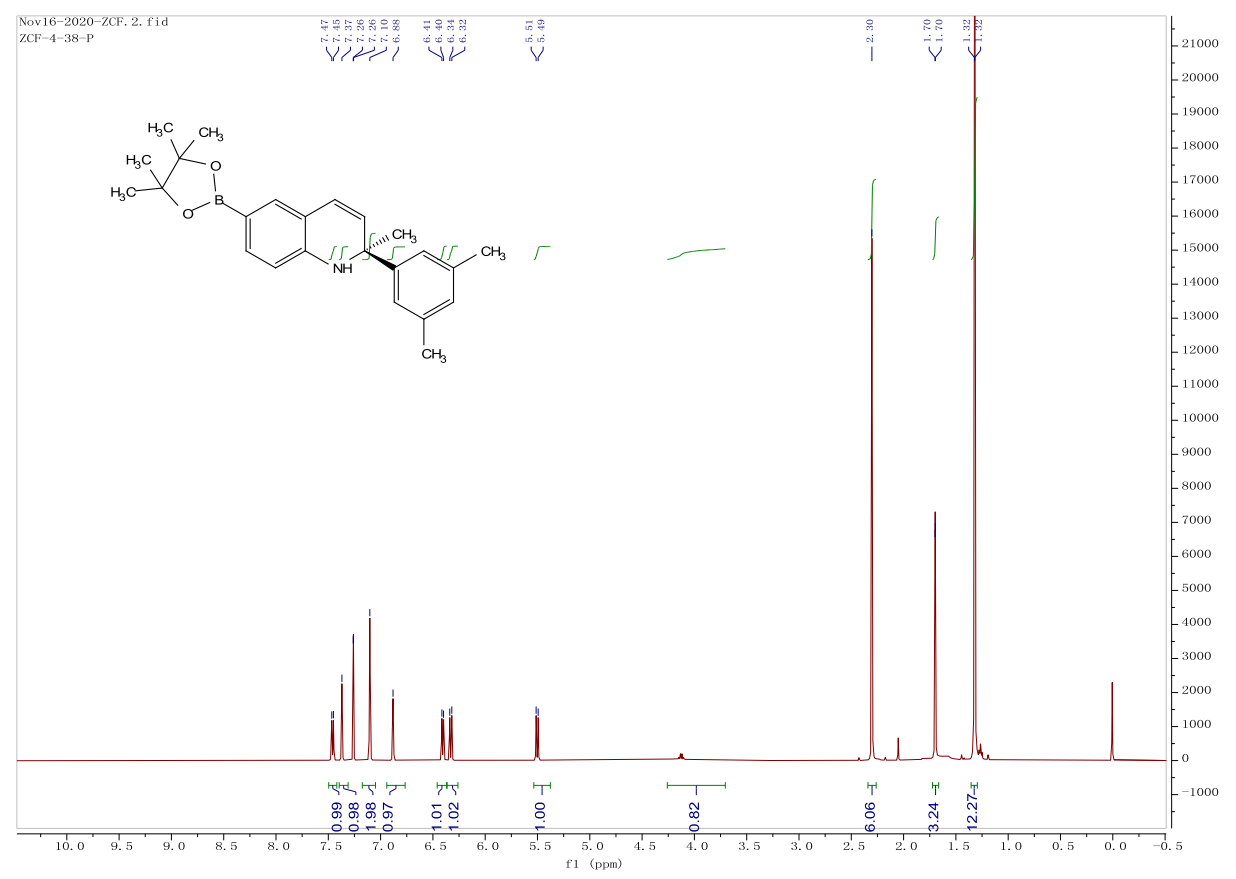

${ }^{1} \mathrm{H}$ NMR(500 MHz, Chloroform- $d$ )

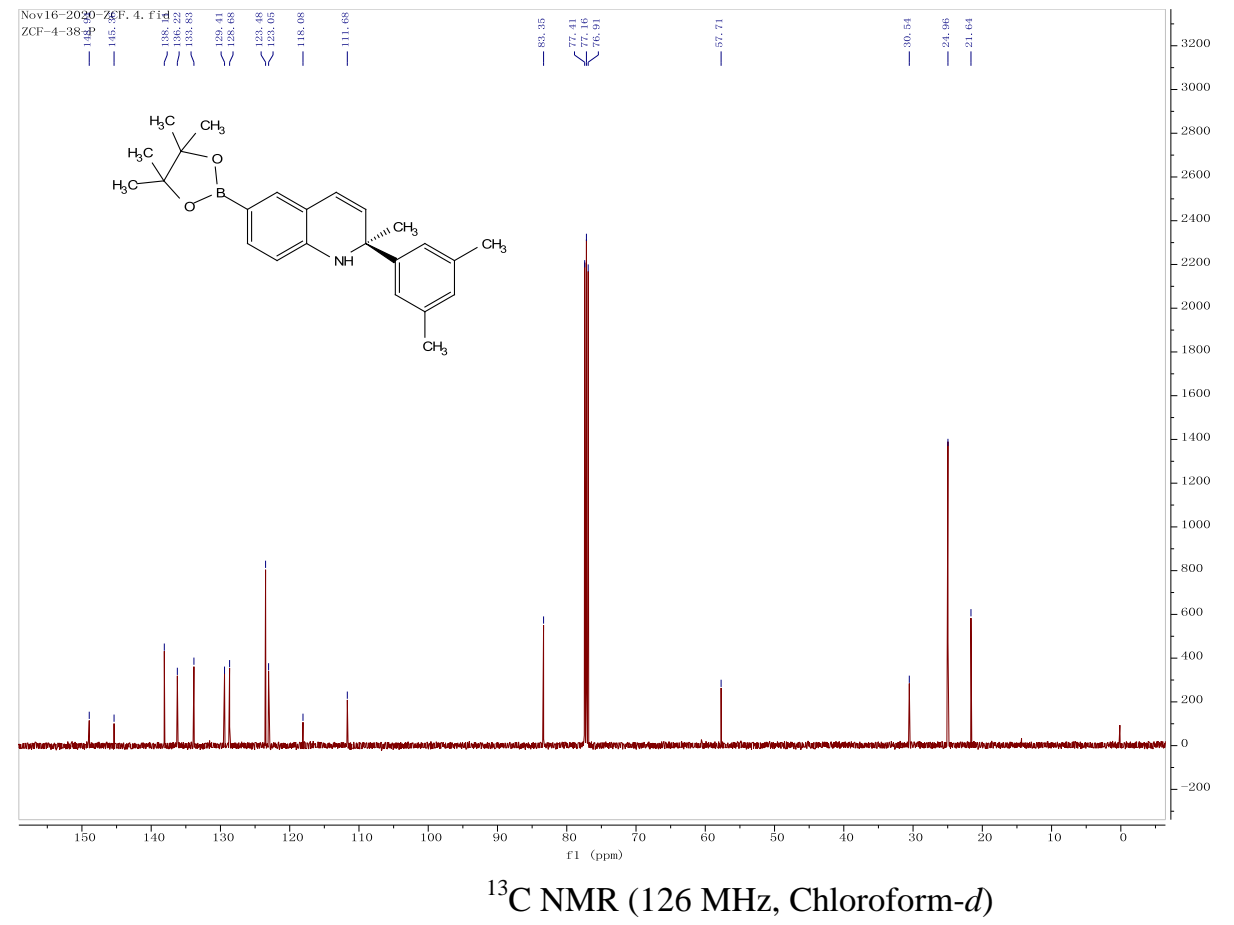


(2R,3R,4R)-6-bromo-3-iodo-4-methoxy-2-methyl-2-phenyl-1,2,3,4-tetrahydroquinoline $((S)-5 a)$<smiles>CO[C@H]1c2cc(Br)ccc2NC(C)(c2ccccc2)[C@H]1I</smiles>

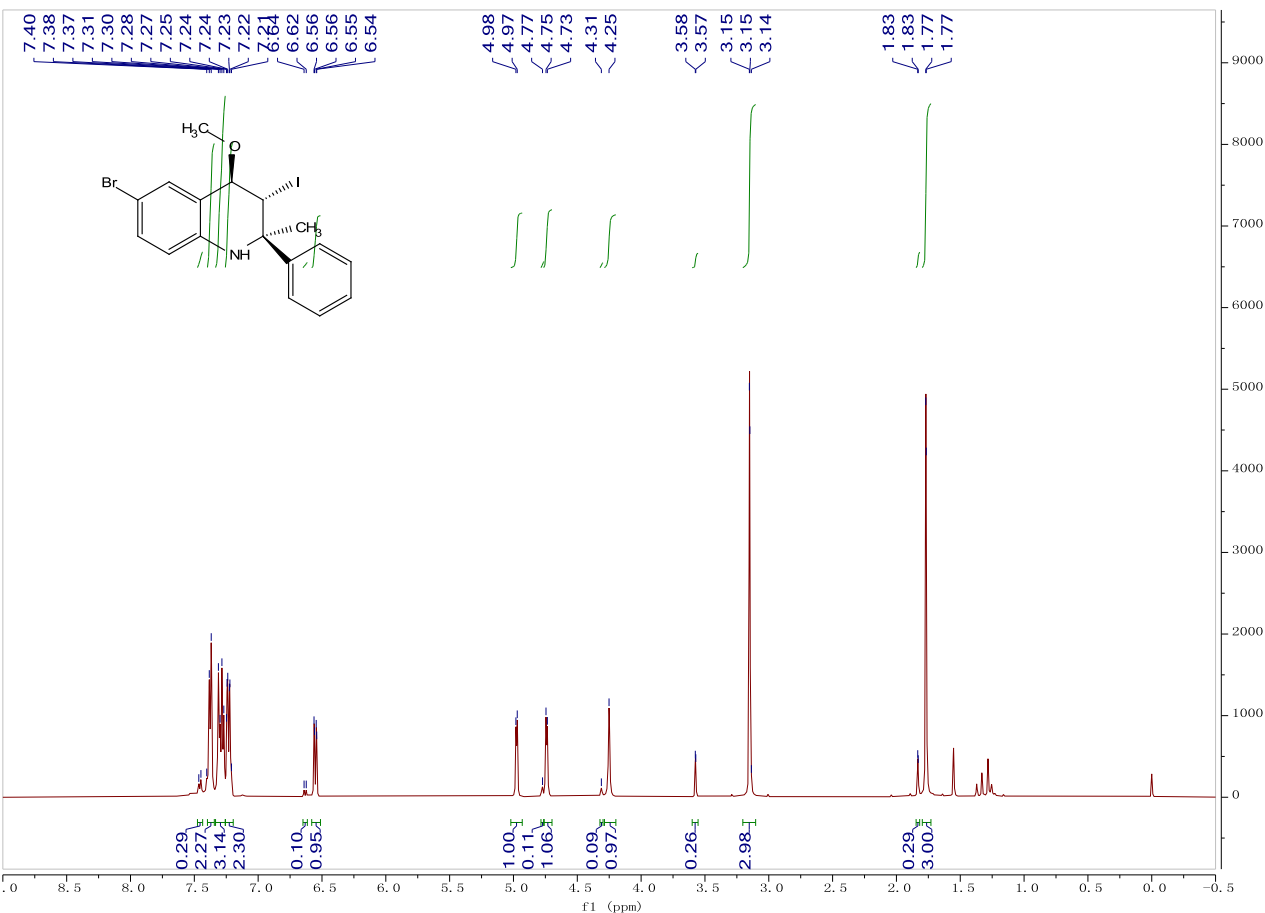

${ }^{1} \mathrm{H} \operatorname{NMR}(500 \mathrm{MHz}$, Chloroform- $d$ )

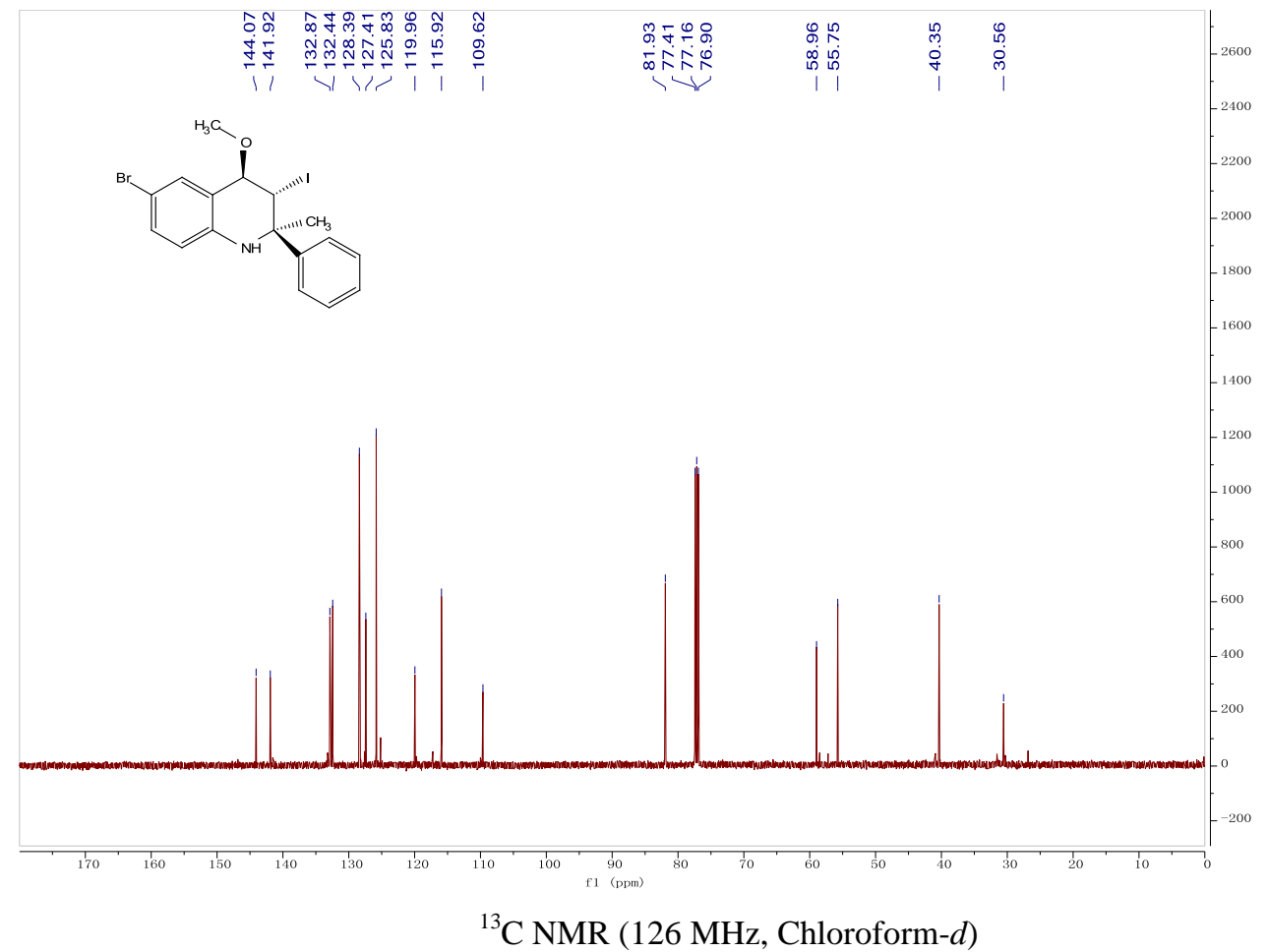


(S)-6-bromo-8-iodo-2-methyl-2-phenyl-1,2,3,4-tetrahydroquinoline $((S)-60)$<smiles>CC(Nc1c(I)cc(Br)cc1I)(c1ccccc1)c1ccccc1</smiles>

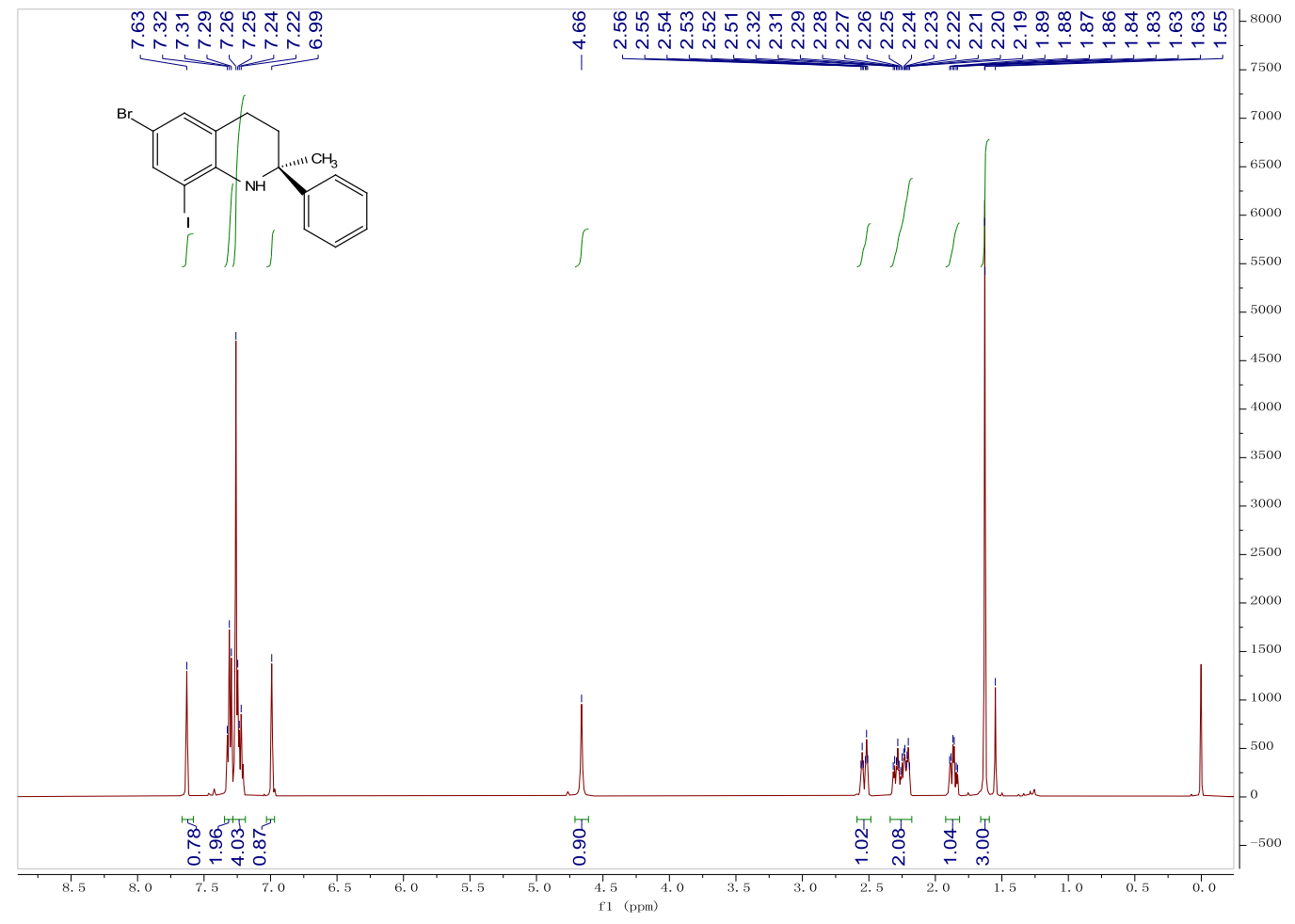

${ }^{1} \mathrm{H}$ NMR(500 MHz, Chloroform- $d$ )

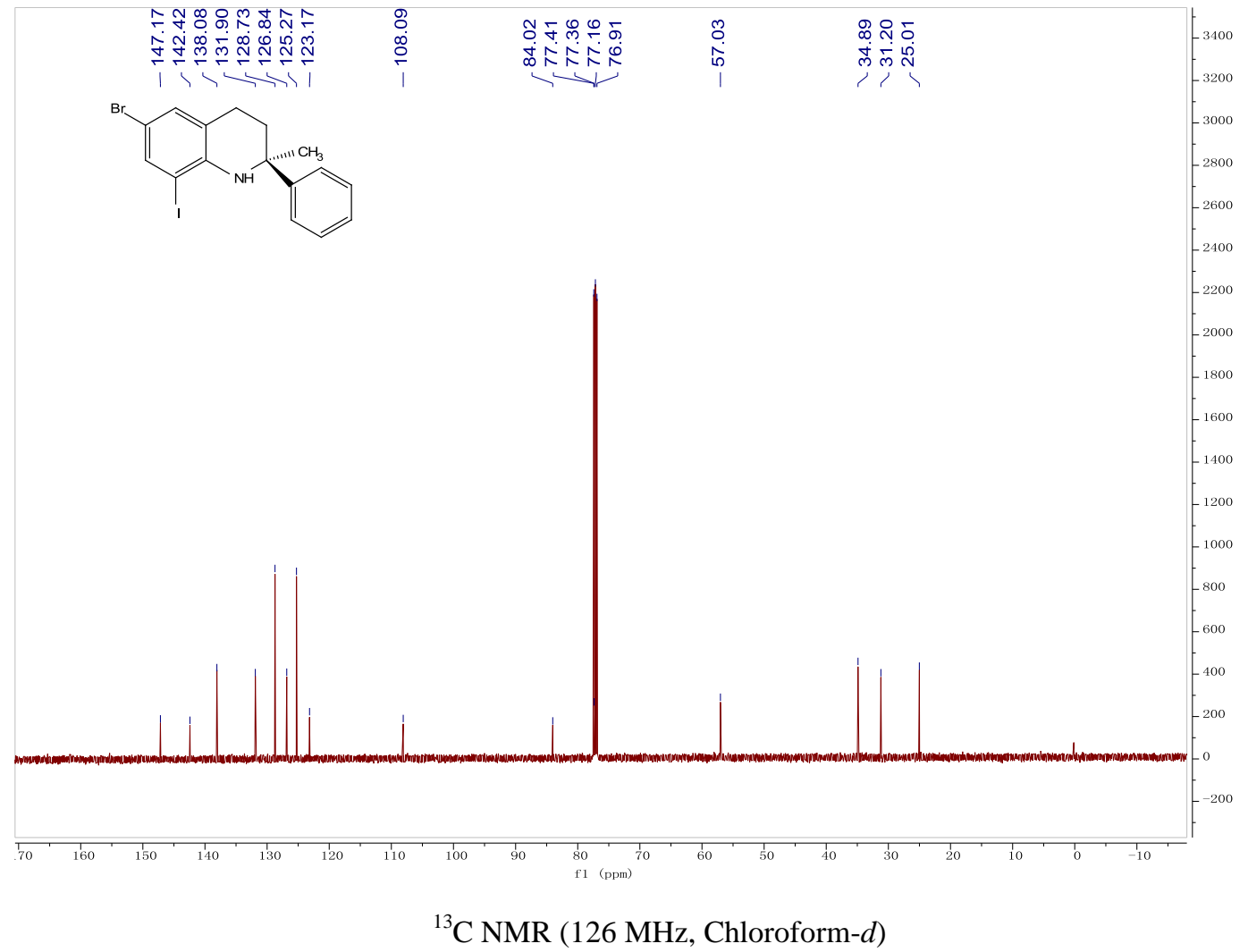

